Aus der Abteilung Kieferorthopädie

(Prof. Dr. med. dent. P. Meyer-Marcotty)

im Zentrum Zahn-, Mund- und Kieferheilkunde

der Medizinischen Fakultät der Universität Göttingen

\title{
Korrelationen zwischen \\ kephalometrischen Werten und dem Knochenangebot intraoraler Spenderregionen für präimplantologische Knochenaugmentationen
}

\author{
INAUGURAL-DISSERTATION \\ zur Erlangung des Doktorgrades \\ für Zahnmedizin \\ der Medizinischen Fakultät der \\ Georg-August-Universität zu Göttingen
}

vorgelegt von

Tayhan Sevinc

aus

Lüdenscheid

Göttingen 2020 
Dekan:

\section{Betreuungsausschuss}

Betreuer/in:

Ko-Betreuer/in:

\section{Prüfungskommission}

Referent/in:

Ko-Referent/in:

Drittreferent/in:

Datum der mündlichen Prüfung:
Prof. Dr. med. W. Brück

PD Dr. med. dent. W. Hahn

Prof. Dr. med. dent. T. Rödig

PD Dr. med. dent. W. Hahn

Prof. Dr. med. dent. T. Rödig

Prof. Dr. Martin Oppermann

23.03.2021 
Hiermit erkläre ich, die Dissertation mit dem Titel "Korrelationen zwischen kephalometrischen Werten und dem Knochenangebot intraoraler Spenderregionen für präimplantologische Knochenaugmentationen" eigenständig angefertigt und keine anderen als die von mir angegebenen Quellen und Hilfsmittel verwendet zu haben.

Göttingen, den

(Unterschrift) 


\section{Inhaltsverzeichnis}

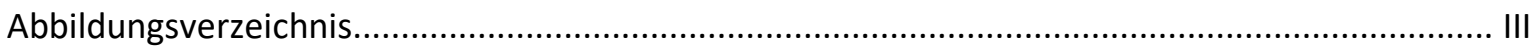

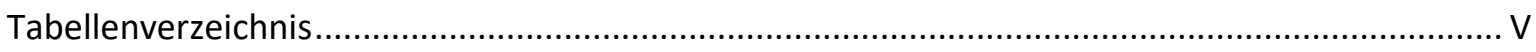

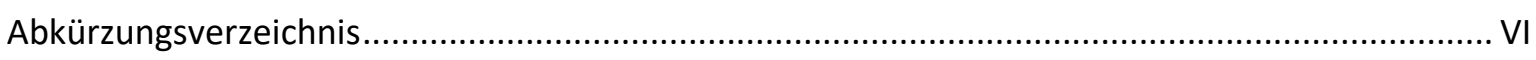

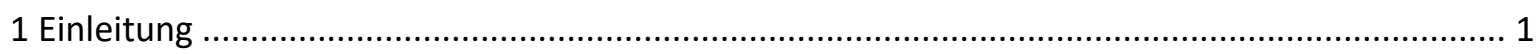

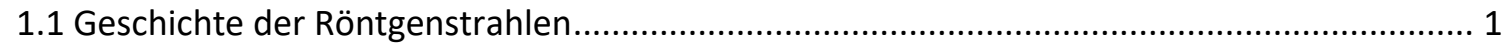

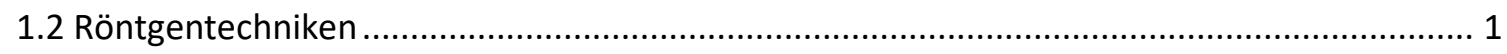

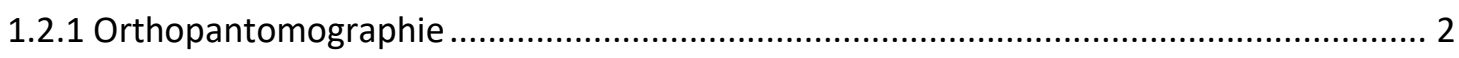

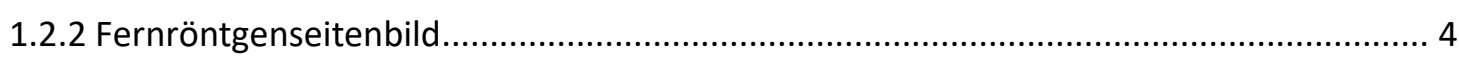

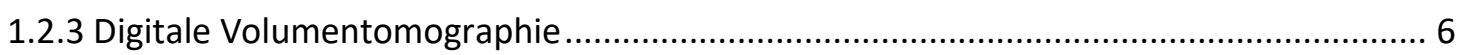

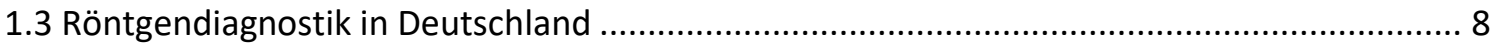

1.4 Präimplantologische Knochenaugmentationen.................................................... 10

1.4.1 Knochenaugmentationsmaterialien......................................................... 10

1.4.2 Intraorale Spenderregionen autogener Knochentransplantate ................................ 12

1.5 Volumetrische Untersuchungen am menschlichen Schädel......................................... 15

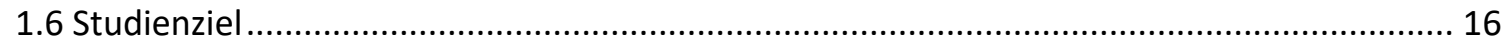

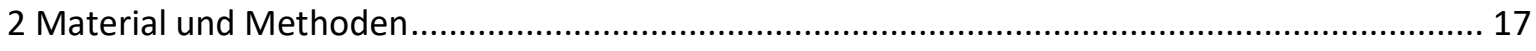

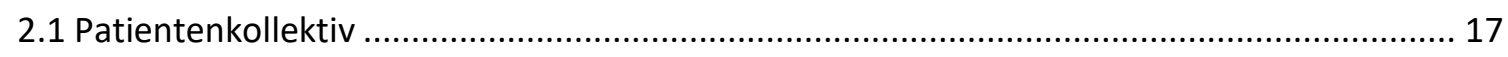

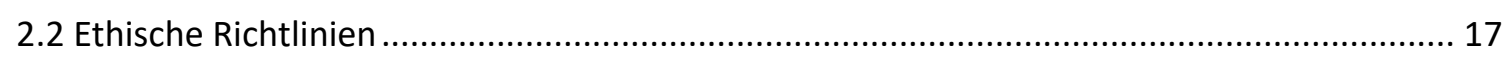

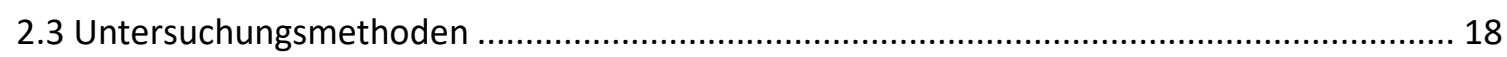

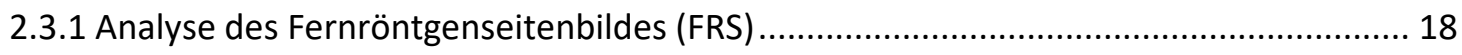

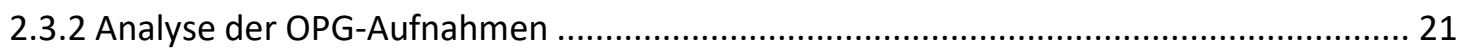

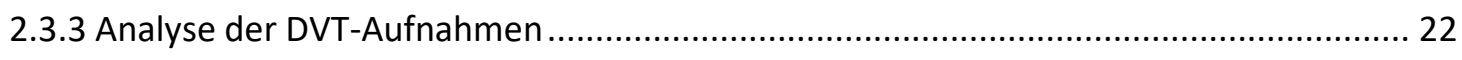

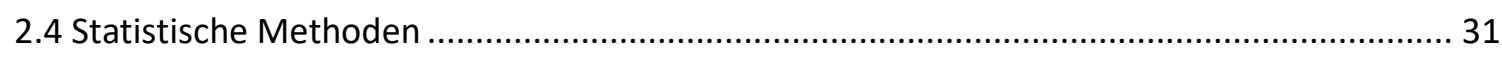

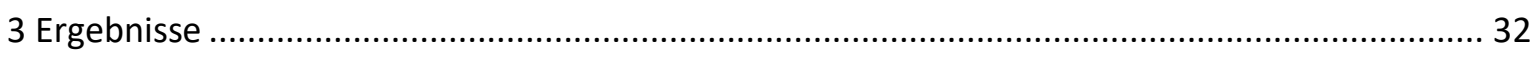

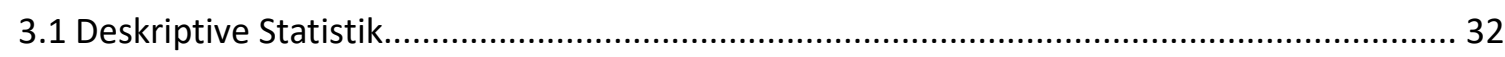

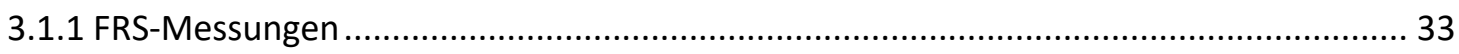

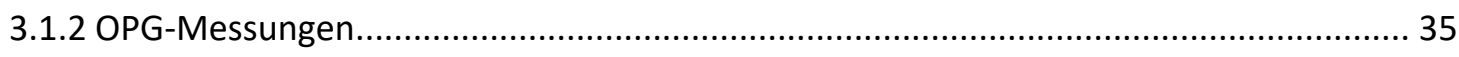

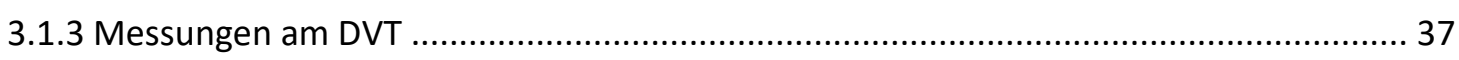

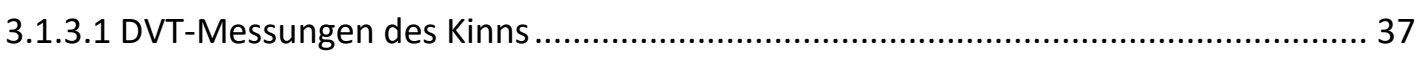

3.1.3.2 DVT-Messungen der retromolaren Region .............................................. 41

3.1.3.3 DVT-Messungen der Crista zygomatico-alveolaris......................................... 45

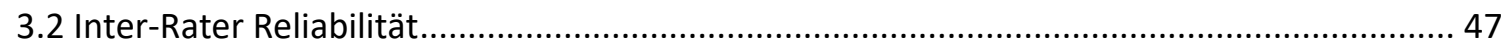

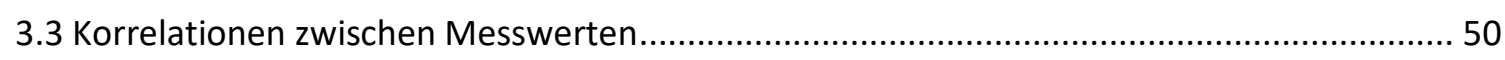

3.3.1 Untersuchung auf Normalverteilung .............................................................. 50

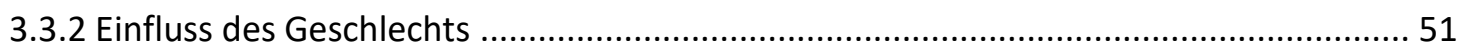


3.3.3 Zusammenhang zwischen kephalometrischen Parametern und Nervverlauf................. 54

3.3.4 Korrelationen zwischen OPG-, FRS- und DVT-Messerwerten ........................................ 55

3.3.4.1 Korrelationen mit DVT-Messwerten im Kinn ............................................................ 55

3.3.4.2 Korrelationen mit DVT-Messwerten der retromolaren Region ................................ 59

3.3.4.3 Korrelationen mit DVT-Messwerten der Crista zygomatico-alveolaris...................... 63

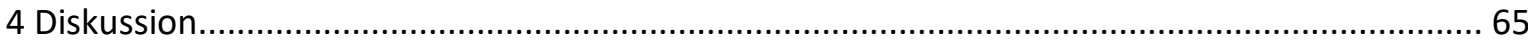

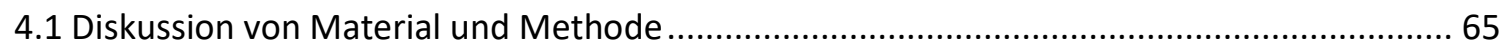

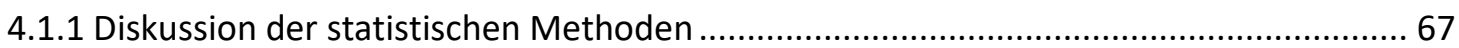

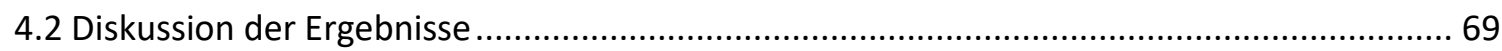

4.2.1 Korrelationen zwischen FRS-Messwerten und Knochendimensionen im DVT ............... 70

4.2.1.1 Korrelationen zwischen FRS-Messerwerten und Knochendimensionen des Kinns. 70

4.2.1.2 Korrelationen zwischen FRS-Messerwerten und Knochendimensionen der

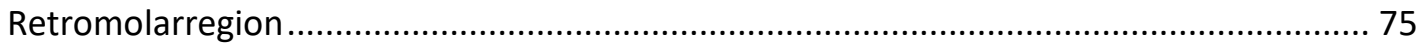

4.2.1.3 Korrelationen mit DVT-Messwerten der Crista zygomatico-alveolaris..................... 79

4.2.2 Korrelationen zwischen OPG-Messwerten und Knochendimensionen im DVT .............. 80

4.2.2.1 Korrelationen zwischen OPG-Messerwerten und Knochendimensionen des Kinns 80

4.2.2.2 Korrelationen zwischen OPG-Messerwerten und Knochendimensionen der

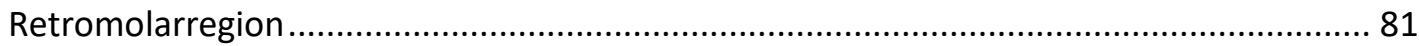

4.2.3 Korrelationen zwischen OPG- und FRS-Messwerten .................................................. 84

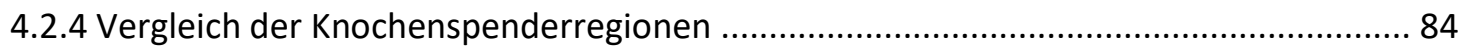

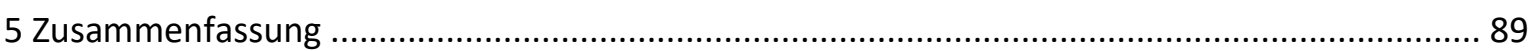

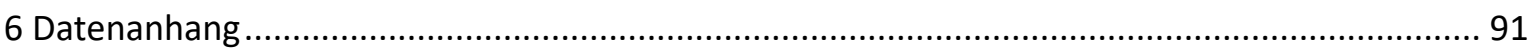

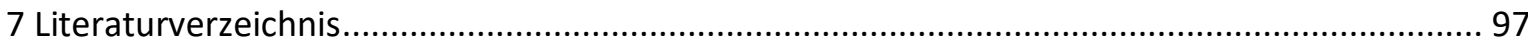

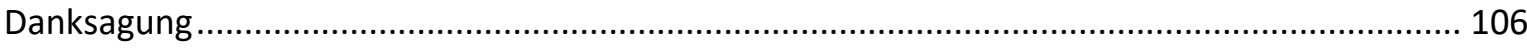




\section{Abbildungsverzeichnis}

Abbildung 1

Abbildung 2

Abbildung 3

Abbildung 4

Abbildung 5

Abbildung 6

Abbildung 7

Abbildung 8

Abbildung 9

Abbildung 10

Abbildung 11

Abbildung 12

Abbildung 13

Abbildung 14

Abbildung 15

Abbildung 16

Abbildung 17

Abbildung 18

Abbildung 19

Abbildung 20

Abbildung 21

Abbildung 22

Abbildung 23

Abbildung 24

Abbildung 25

Abbildung 26

Abbildung 27

Abbildung 28

Abbildung 29

Abbildung 30

Abbildung 31

Abbildung 32

Abbildung 33

Abbildung 34

Abbildung 35

Abbildung 36

Abbildung 37

Abbildung 38

OPG eines Patienten 3

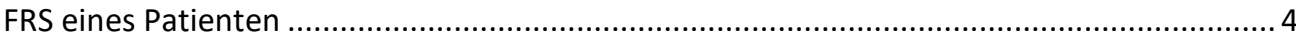

Softwaregestützte Darstellung der mittels DVT gewonnenen Daten in drei Ebenen mit zusätzlichem virtuellem 3D-Modell

Anzahl der Röntgenuntersuchungen pro Einwohner und Jahr von 1996 bis 2012 ................ 8

Prozentualer Anteil der Fachrichtungen an durchgeführten Röntgenaufnahmen insgesamt

\& Prozentualer Anteil an der kollektiven Dosis

Dreidimensionale DVT-Rekonstruktion des Schädels mit farblich markierten intraoralen Entnahmeregionen

FRS mit eingezeichneten kephalometrischen Variablen ............................................... 18

Zu untersuchende morphologische Eigenschaften des mandibulären Unterrandes ............ 19

Form des Kondylus und canalis mandibularis mit entsprechendem "Score“...................... 20

Zuordnung kephalometrischer Messwerte zu einem ",Score“............................................. 20

OPG mit Einzeichnungen der vorgenommenen Messungen ............................................. 21

Axiale Ansicht des Kinns in einer DVT-Aufnahme mit Sicherheitsmarkierungen und horizontaler Messung

Sagittalschnitt des Kinns in einer DVT-Aufnahme mit Sicherheitsmarkierungen und Breiten-

(CS) bzw. Höhenmessungen (CV) des Knochens

Dreidimensionale DVT-Rekonstruktion des Kinns mit Darstellung aller gemessenen Strecken

Sagittalschnitt der retromolaren Region in der DVT-Aufnahme

Koronare Ansicht der retromolaren Region im DVT mit rot markiertem Nervus alveolaris inferior und gemessenen Strecken

Messung der bukkalen Knochenwand (MR) in der axialen Ansicht im DVT .........................2 24

Dreidimensionale DVT-Rekonstruktion der rechten retromolaren Region .......................... 25

Markierte Transplantatbreite der Crista zygomatico-alveolaris in der Axialansicht der DVT-

Aufnahme

Koronare Ansicht der Crista zygomatico-alveolaris in der DVT-Aufnahme, parallel zu ihrem Umschlagspunkt

Schematische Darstellung der Messungen an der Crista zygomatico-alveolaris ................. 27

Schematische Darstellung der Messungen im Kinn.............................................................. 28

Schematische Darstellung der Messungen im retromolaren Knochenblock....................... 28

Axiale Ansicht der Mandibula im DVT mit markiertem Abstand von $5 \mathrm{~mm}$ distal des letzten Molaren .....

Koronare Ansicht der Mandibula im DVT. Der lingual verlaufende Nervus alveolaris inferior ist rot markiert

Koronare Ansicht der Mandibula im DVT. Der bukkal verlaufende Nervus alveolaris inferior

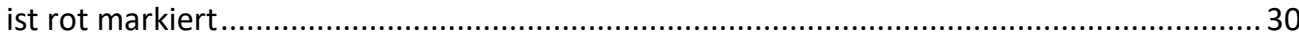

Histogramm zur Darstellung der Verteilung des Patientenalters ...................................... 32

Box-Whisker-Plot des FRS Index in Prozent [\%] .............................................................. 33

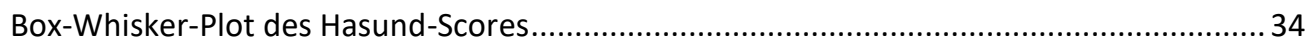

Box-Whisker-Plot der im FRS gemessenen Winkel in Grad $\left[{ }^{\circ}\right]$.............................................. 34

Box-Whisker-Plot des im OPG gemessenen WS1 in [mm] .............................................. 35

Box-Whisker-Plot des im OPG gemessenen WS7 in [mm] ............................................ 36

Box-Whisker-Plot des im OPG gemessenen Kieferwinkels (Ang. Mand.) in Grad [ $\left.{ }^{\circ}\right]$............. 36

Box-Whisker-Plot der transversalen Knochenbreite des Kinns in drei Lagen ( $\mathrm{CH} 1$ bis $\mathrm{CH} 3)$. 39 Box-Whisker-Plot der vertikalen Knochenhöhe des Kinns an drei Positionen (CV1 bis CV3) 39 Box-Whisker-Plot der Knochendicke des Kinns an neun Positionen (CS1 bis CS9)............... 40 Box-Whisker-Plot des errechneten entnehmbaren Knochenvolumens im Kinn (Vchin)....... 40

Säulendiagramm des retromolaren Nervverlaufs in der Mandibula 
Abbildung 39 Box-Whisker-Plot der retromolaren vertikalen Knochenhöhe an drei Positionen (MN1 bis MN3)

Abbildung 40 Box-Whisker-Plot der retromolaren Knochenbreite an neun Positionen (MW1 bis MW9).. 43

Abbildung 41 Box-Whisker-Plot der vestibulären Knochendicke an beiden Wurzeln des zweiten Unterkiefermolaren (MR1 und MR2)

Abbildung 42 Box-Whisker-Plot des errechneten entnehmbaren retromolaren Knochenvolumens (Vretro)

Abbildung 43 Box-Whisker-Plot der Knochendicke an neun Messpositionen der Crista zygomaticoalveolaris (ZA1 bis ZA9) .................................................................................. 46

Abbildung 44 Box-Whisker-Plot des errechneten entnehmbaren Knochenvolumens an der Crista zygomatico-alveolaris (Vcrista) ............................................................................ 46

Abbildung 45 Shapiro-Wilk-Test inklusive Histogramm der Messdifferenzen beider Rater bei Messungen

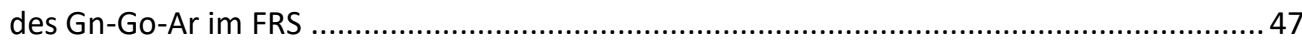

Abbildung 46 Bland-Altman-Plot zur Ermittlung der Interrater-Reliabilität bei Messungen des

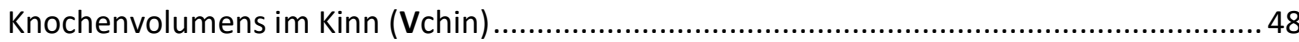

Abbildung 47 Shapiro-Wilk-Test auf Normalverteilung für die sagittale Knochendicke im Kinn (CS6) .......50 Abbildung 48 Box-Whisker-Plot der Knochenvolumina verschlüsselt nach Geschlecht und Entnahmeareal

Abbildung 49 Scatterplot der Pearson-Korrelation zwischen dem Winkel ML-NSL und der transversalen Breite des knöchernen Kinns CH3 ........................................................................ 56

Abbildung 50 Scatterplot der Pearson-Korrelation zwischen dem Winkel ML-NSL und der Knochenhöhe des Kinns CV3 .................................................................................................. 57

Abbildung 51 Scatterplot der Pearson-Korrelation zwischen den Strecken WS1 und CS4 ........................58

Abbildung 52 Scatterplot der Pearson-Korrelation zwischen der Strecke WS1 und dem Knochenvolumen des Kinns Vchin .............................................................................................................. 59

Abbildung 53 Scatterplot der Pearson-Korrelation zwischen der Strecke WS7 und der Höhe des retromolaren Knochenblockes MN2 …................................................................6 60

Abbildung 54 Scatterplot der Pearson-Korrelation zwischen der Strecke WS7 und dem retromolaren Knochenvolumen Vretro .......................................................................................... 61

Abbildung 55 Scatterplot der Pearson-Korrelation zwischen dem Gn-Go-Ar und dem Ang. Mand............62 Abbildung 56 Scatterplot der Pearson-Korrelation zwischen dem ANB und der Höhe des retromolaren Knochenblockes MN2 ........................................................................................... 63

Abbildung 57 Scatterplot der Pearson-Korrelation zwischen dem Knochenvolumen der Crista zygomaticoalveolaris Vcrista und dem retromolaren Knochenvolumen Vretro .................................6 64

Abbildung 58 Schematische Darstellung des möglichen Einflusses der Distanz zwischen vorderer und hinterer Gesichtshöhe auf die Winkel ML-NL und ML-NSL ............................................. 72

Abbildung 59 DVT-Rekonstruktion der Mandibula mit untersuchten Messpunkten (CS1 bis CS9) und den entsprechenden Pearson Korrelationen zu dem ML-NSL............................................... 72

Abbildung 60 Schematische Darstellung des Knochenwachstums im Kinn als Sagittalschnitt mit markierten Messpunkten CS4 und CS6 .................................................................. 73

Abbildung 61 DVT-Rekonstruktion der Mandibula mit untersuchten Messpunkten ( $\mathrm{CH} 1$ bis $\mathrm{CH} 3$ ) und den entsprechenden Pearson Korrelationen zu dem ML-NSL................................................ 74

Abbildung 62 DVT-Rekonstruktion der Mandibula mit untersuchten Messungen (CV1 bis CV3) und den entsprechenden Pearson Korrelationen zu dem ML-NSL............................................... 75

Abbildung 63 Dreidimensionale DVT-Rekonstruktion der rechten retromolaren Region zweier Patienten

Abbildung 64 Koronare Ansicht des retromolaren Knochenblocks im DVT mit markierter Linea mylohyoidea in drei zunehmend distalen Schnittebenen.

Abbildung 65 Dreidimensionale DVT-Rekonstruktion des Schädels mit eingezeichneten Hauptkraftlinien

Abbildung 66

Sagittalschnitt durch den mittleren Unterkiefer Incisivus im DVT. 


\section{Tabellenverzeichnis}

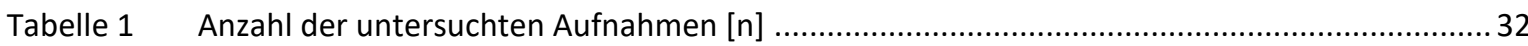

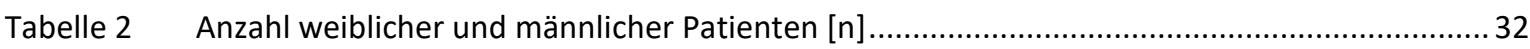

Tabelle 3 Deskriptive Statistik für sämtliche Messungen im FRS ........................................................... 33

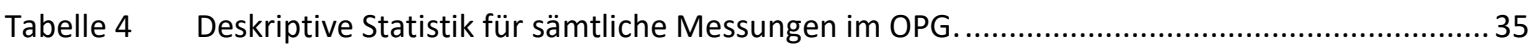

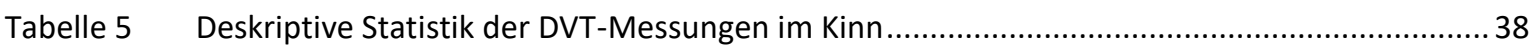

Tabelle 6 Deskriptive Statistik der retromolaren Messungen im DVT................................................... 41

Tabelle 7 Anzahl lingual und bukkal verlaufender Nervenpaare im retromolaren Bereich ...................... 42

Tabelle 8 Deskriptive Statistik der DVT-Messungen an der Crista zygomatico-alveolaris......................... 45

Tabelle 9 Ergebnisse des Bland-Altman-Plots zur Darstellung der Inter-Rater Reliabilität ....................... 49

Tabelle 10 T-test für die Untersuchung des Geschlechts als unabhängige und $\mathrm{CH} 2$ bzw. $\mathrm{CH} 3$ als abhängige

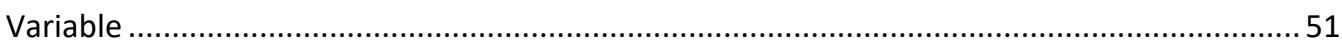

Tabelle 11 Univariate lineare Regression mit dem Geschlecht als unabhängige Variable und $\mathrm{CH} 2$ als abhängige Variable....................................................................................................... 51

Tabelle 12 Univariate lineare Regression mit dem Geschlecht als unabhängige Variable und CH3 als

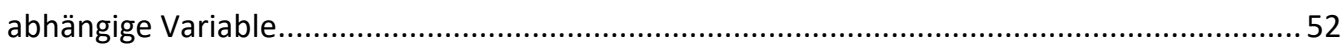

Tabelle 13 T-test für die Untersuchung des Geschlechts als unabhängige und die Knochenvolumina als

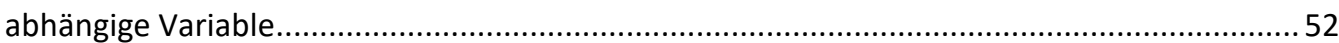

Tabelle 14 Tabelle zum Chi-Quadrat-Test zur Prüfung einer möglichen Abhängigkeit des Nervverlaufs von

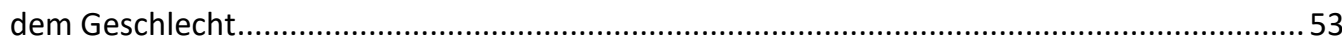

Tabelle 15 T-tests für die Untersuchung der kephalometrischen Messungen als unabhängige Variable und den Nervverlauf als abhängige Variable ..................................................................... 54

Tabelle 16 Logistische Regression mit backward removal Algorithmus zur Untersuchung des Einflusses kephalometrischer Parameter auf den Nervverlauf in bukkaler Position................................. 54

Tabelle 17 Pearson-Korrelationen zwischen FRS-Messungen und DVT-Messungen im Kinn ......................55

Tabelle 18 Pearson-Korrelationen zwischen OPG-Messungen und DVT-Messungen im Kinn ....................57

Tabelle 19 Pearson-Korrelationen zwischen OPG-Messungen und DVT-Messungen in der retromolaren

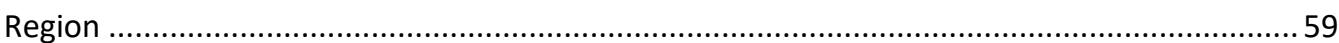

Tabelle 20 Pearson-Korrelationen zwischen FRS-Messungen und DVT-Messungen der retromolaren Region, sowie zwischen FRS-Messungen und OPG-Messungen .........................................61

Tabelle 21 Pearson-Korrelationen zwischen DVT-Messungen der Crista zygomatico-alveolaris und DVTMessungen der retromolaren Region ............................................................................ 64

Tabelle 22 Durchschnittlich gemessene FRS Werte und deren Sollwerte nach Hasund............................69

Tabelle 23 Totale und relative (\%) Sollwert-Abweichungen der FRS-Messwerte im untersuchten Patientenkollektiv 


\section{Abkürzungsverzeichnis}

BMP

$\mathrm{CH}$

CS

CT

CV

DICOM

DVT

FRS

$\mathrm{MN}$

MW

OPG

Vchin

Vcrista

Vretro

WS

ZA
Bone Morphogenic Protein

Chin horizontal (horizontale Breite des Kinns)

Chin sagittal (sagittale Knochendicke des Kinns)

Computertomographie

Chin vertical (vertikale Höhe des Kinns)

Digital Imaging and Communications in Medicine

Digitale Volumentomographie

Fernröntgenseitenbild

Molar nerve (Abstand zw. Knochenoberfläche und Nerv)

Molar width (Breite des retromolaren Knochens)

Orthopantomographie

Knochenvolumen des Kinns

Knochenvolumen der Crista zygomatico-alveolaris

Knochenvolumen des retromolaren Areals

Wurzelspitze (Abstand zw. Apex und Unterkieferbasis)

Zygomatico-alveolaris (Knochendicke der Crista) 


\section{Einleitung}

\subsection{Geschichte der Röntgenstrahlen}

Mit Entdeckung der Röntgenstrahlen durch Wilhelm Conrad Röntgen am 8. November 1895, erlangten diese schnell einen hohen Stellenwert in der Medizin. Der atraumatische Einblick in den Patienten revolutionierte die medizinische Diagnostik und Therapie, sodass heute kaum ein medizinisches Fachgebiet existiert, das keinen Gebrauch von dieser ionisierenden Strahlung macht. Computertechnologische Fortschritte haben die Anwendung zusätzlich erleichtert und erweitert. Digitale Röntgenaufnahmetechniken ermöglichen, durch den Gebrauch von elektronischen Sensoren anstelle von konventionellen Röntgenfilmen mit chemischen Entwicklungsprozessen, neben einer nachträglichen Bildbearbeitung mit geringem Aufwand, eine platzsparende Aufbewahrung von Daten und das schnellere Teilen dieser Aufnahmen mit Fachkollegen (van der Stelt 2005). Zudem wurden durch die stark erhöhte Rechenleistung von Computern dreidimensionale Röntgentechnologien wie die CT oder DVT ermöglicht, die durch eine hohe Informationsquantität und räumliche Darstellung detailliertere Planungen operativer Eingriffe ermöglichen können als zweidimensionale Aufnahmen (Kaeppler und Mast 2012; Akiba 2013).

\subsection{Röntgentechniken}

Generell lassen sich zwei- von dreidimensionalen Aufnahmen unterscheiden, wobei letztere, aufgrund der höheren Strahlenbelastung, erst bei Fragestellungen zum Einsatz kommen, die durch zweidimensionale Aufnahmen nicht vollständig beantwortet werden können (Grünheid et al. 2012; Pauwels 2015). Folgend werden die Röntgenverfahren dargestellt, die im Rahmen dieser Arbeit angewendet und verglichen wurden. 


\subsubsection{Orthopantomographie}

Bei dem OPG (Orthopantomogramm) handelt es sich um eine Panoramaschichtaufnahme, die hauptsächlich das Viszerokranium abbildet. Simultan zu dem Bestrahlungsvorgang durch die Röntgenröhre, umfahren Strahlenquelle und der gegenüberliegende Röntgenfilm oder Sensor den unbewegten Patienten in einem Kreissegment, das dem Zahnbogenverlauf angepasst ist. Es erfolgt eine sukzessive Durchleuchtung mit einem senkrechten, keilförmigen Strahlenbündel. Da die Strahlenquelle sich schneller als der Sensor bzw. Film bewegt, kann durch aneinandergereihte, streifenförmige Belichtungen schließlich eine vollständige Belichtung des Sensors bzw. Films erfolgen. Hierdurch entsteht ein zusammengesetztes Panorama-Abbild. Die scharf abgebildete Schicht wird durch die Lage der Fokusebene bestimmt, sodass die restlichen Areale in der Unschärfe liegen und entsprechend weniger definiert abgebildet werden. Eine Vielzahl anatomischer Strukturen kann so erfasst werden. Hierzu gehören der Ober- und Unterkiefer samt Alveolarfortsatz, sämtliche Zähne, beide Kiefergelenke und die Kieferhöhlen. Zusätzlich werden supralaryngeale Bereiche des Halses belichtet, sodass die Möglichkeit von Zufallsbefunden bei z. B. arteriosklerotischen Veränderungen der Carotiden besteht (Ohba et al. 2003). Auch wenn die übersichtliche Darstellung dieser zahlreichen Strukturen ein breites Anwendungsspektrum für das OPG schafft (van der Stelt 2016), ist zu bedenken, dass das OPG nicht für jede zahnärztliche Fragestellung das beste Röntgenverfahren darstellt (Douglass et al. 1986; Valachovic et al. 1986). Verfahrenstechnisch bedingt, kommt es zu charakteristischen Überlagerungen einzelner Strukturen, wie z. B. der Halswirbelsäule im Bereich der Unterkiefer Frontzähne oder des harten Gaumens im Bereich der Oberkiefer Zahnwurzeln. Eine diagnostische Aussage zu den Kiefergelenken nur anhand einer OPG-Aufnahme ist nur mit Einschränkungen möglich (Dahlström und Lindvall 1996; Fallon et al. 2006) und die Detektion approximaler kariöser Läsionen ist mit dem OPG weniger zuverlässig als mit Bissflügel- oder Einzelzahnaufnahmen (Hurlburt und Wuehrmann 1976; Kamburoglu et al. 2012; Terry et al. 2016).

Trotz dieser Einschränkungen wird das OPG aufgrund der großen Übersicht bei geringerem Zeitaufwand und geringerer Strahlendosis im Vergleich zu DVT- und CT-Aufnahmen als zahnmedizinisch-radiologisches Routinediagnostikum regelmäßig eingesetzt. 


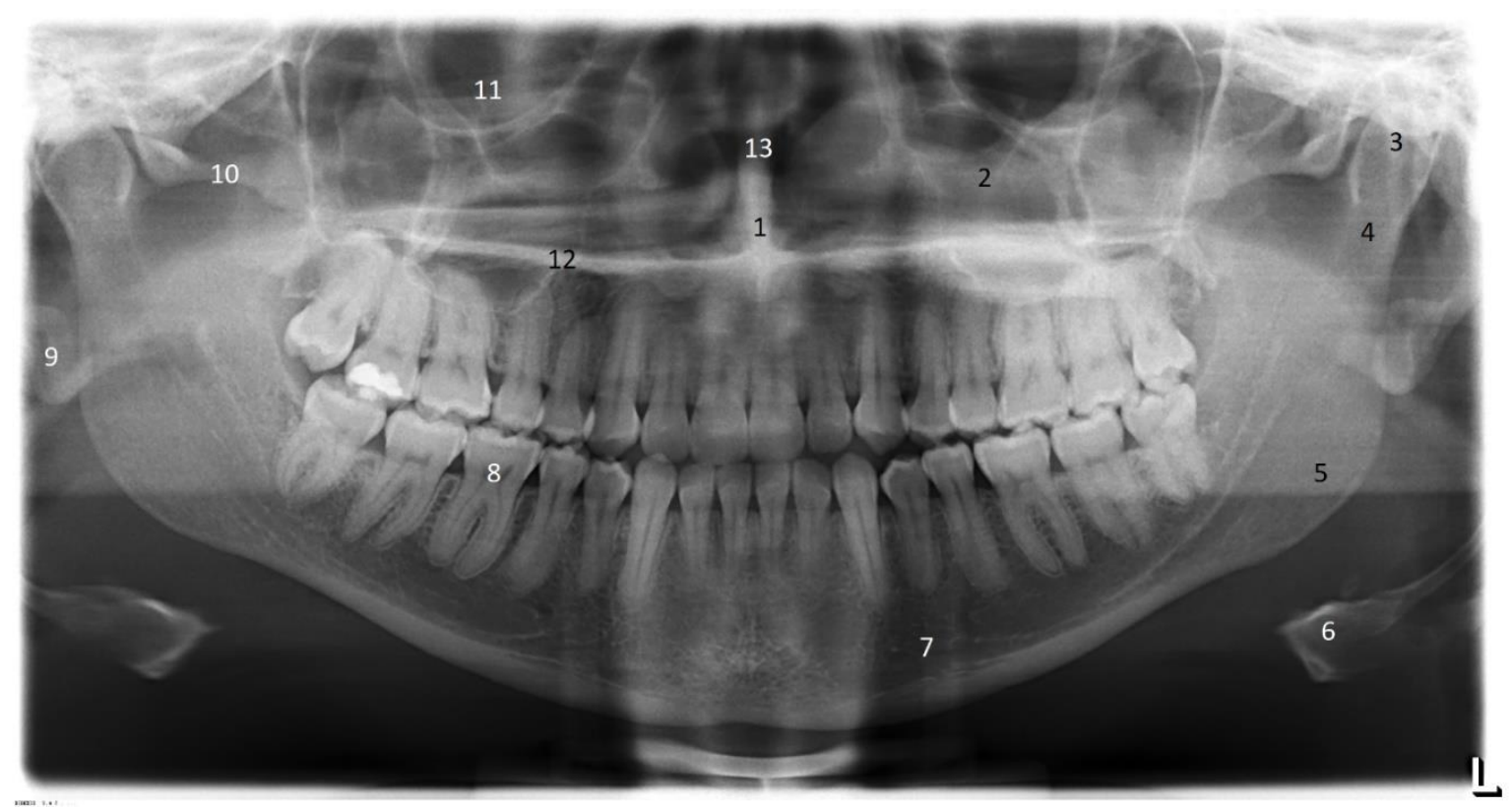

Abbildung 1

OPG eines Patienten: 1 Spina nasalis anterior 2 Sinus maxillaris 3 Caput mandibulae 4 Collum mandibulae 5 Mandibula (bzw. Angulus mandibulae) 6 Os hyoideum 7 Foramen mentale 8 Zahn 469 Ohr 10 Arcus zygomaticus 11 Orbita 12 Palatum durum 13 Septum nasi. (Klinik für Mund-, Kiefer- und Gesichtschirurgie Universitätsklinikum Göttingen) 


\subsubsection{Fernröntgenseitenbild}

Während der Kopf des Patienten in einer Arretierungsvorrichtung fixiert wird, befindet sich zu einer Seite des Patienten die Strahlenquelle mit einem Strahlenverlauf im rechten Winkel zur Sagittalebene. Ein Abstand von 1,5 m soll einen möglichst parallelen Strahlengang ermöglichen. Auf der Gegenseite befindet sich parallel zu der Sagittalebene der Röntgenfilm bzw. Sensor mit möglichst geringem Abstand zu dem Kopf. Hierdurch wird die VergröBerung des Abbildes und die Entstehung von Doppelkonturen minimiert (Segner und Hasund 1998). Strahlenquelle und Sensor sind mit vertikalen Schlitzblenden versehen, die sich während der Bestrahlung synchron bewegen. Es erfolgt eine sukzessive schlitzförmige Belichtung des Filmes bzw. Sensors. Die Belichtung erzeugt somit eine laterale RöntgenProfilaufnahme, die neben den knöchernen Strukturen des Schädels und der Halswirbelsäule auch das Weichteilprofil bei Gebrauch von Weichteilfiltern abbildet (Pasler 2017).

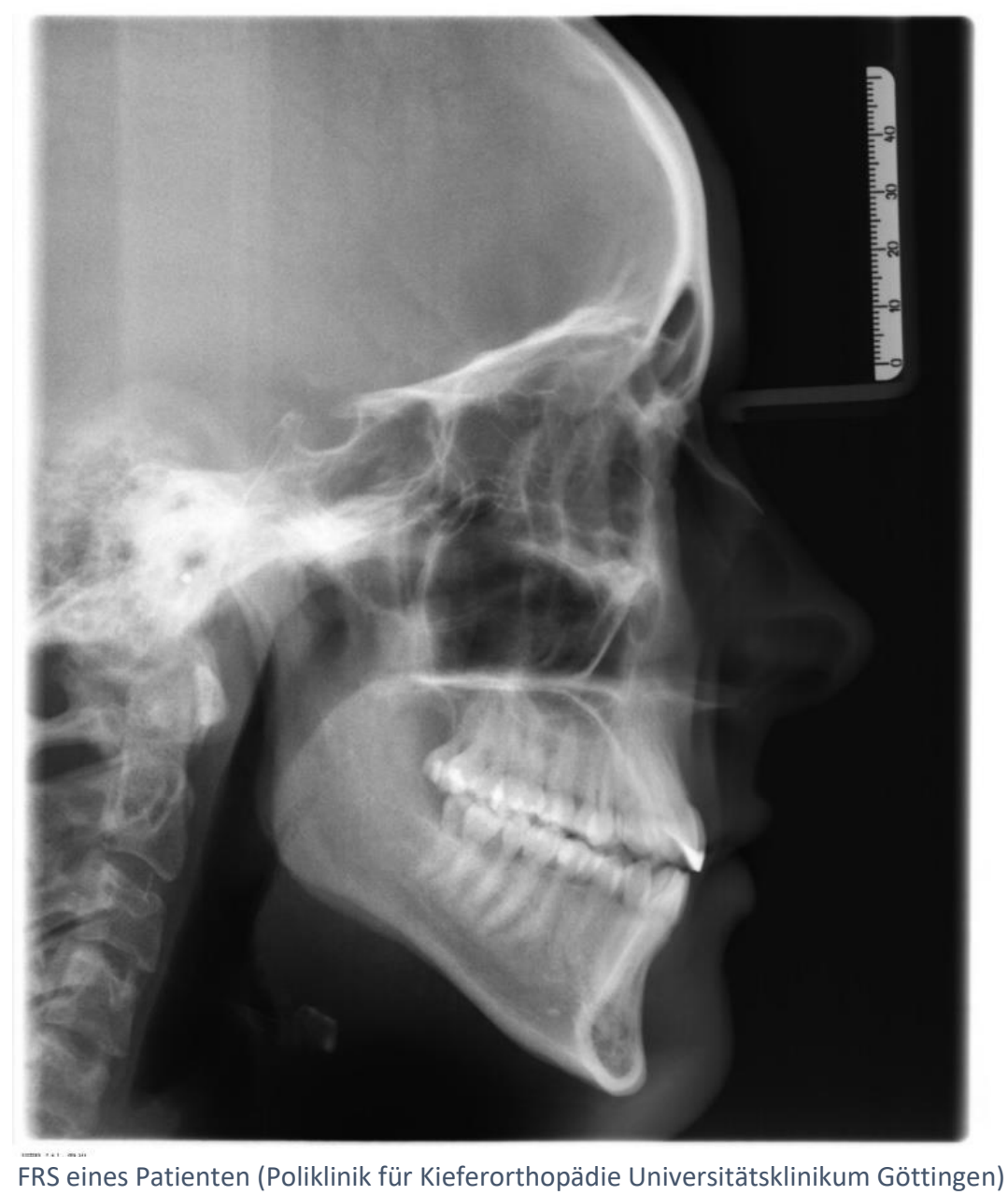

Mit Hilfe des FRS (Fernröntgenseitenbild) als kieferorthopädisches Routinediagnostikum können kephalometrische Analysen durchgeführt werden. Hierbei werden fest definierte 
und eindeutig reproduzierbare Referenzpunkte markiert, die der Konstruktion von Linien und Winkeln dienen (Segner und Hasund 1998). Eine standardisierte Röntgenaufnahme und Analyse ermöglicht Vergleiche sowie Beurteilungen zu dem Behandlungsverlauf und abschließend -erfolg (Broadbent 1931; Hofrath 1931; Wedler et al. 2006). Der Vergleich dieser Messungen mit „individuellen Normen“ erlaubt schließlich eine diagnostische Aussage zu möglichen Kiefer- und Zahnfehlstellungen (Segner und Hasund 1998).

Computertechnologische Fortschritte ermöglichen eine digitale Analyse des FRS. Neben einer schnelleren Auswertung der Röntgenaufnahmen, werden statistische Auswertungen und Wachstumsanalysen sowie -vorhersagen erleichtert (Ricketts 1972; Celik et al. 2009). Zusätzlich kann die computergestützte Auswertung von Fernröntgenseitenbildern zu einer Minimierung von Mess- und Übertragungsfehlern beitragen (Beckmann et al. 1983).

Durch eine verzerrungsarme Darstellung sind mit dem FRS als Routinediagnostikum der Kieferorthopädie neben quantifizierbaren Diagnosen von Kieferfehlstellungen, Aussagen über den Wachstumstyp des Patienten möglich. Diese Informationen können einen signifikanten Einfluss auf die Therapieplanung haben und sind fester Bestandteil der kieferorthopädischen Diagnostik und Therapie (Bruks et al. 1999; Devereux et al. 2011). 


\subsubsection{Digitale Volumentomographie}

Als häufigstes dreidimensionales Bildgebungsverfahren in der Zahnmedizin wird die DVT (Digitale Volumentomographie) gegenüber der traditionellen CT (Computertomographie) aufgrund der geringeren Strahlendosis, kompakteren Bauweise und zunehmend sinkenden Anschaffungskosten bevorzugt (Mozzo et al. 1998; Arai et al. 1999; Loubele et al. 2009). Analog zu der CT werden aus multiplen Röntgen-Projektionsaufnahmen dreidimensionale Volumina errechnet. Während die CT Gebrauch von einem zweidimensionalen Fächerstrahl macht, der das Untersuchungsobjekt aus mehreren Richtungen durchleuchtet, ist es bei der DVT ein dreidimensionales, konusförmiges Strahlenbündel (cone beam). So kann eine Computersoftware die mittels DVT gewonnenen Daten in drei Schnittebenen darstellen und ein virtuelles 3D-Modell erzeugen. Der hieraus resultierende Datensatz im DICOM-Format kann je nach Größe des Untersuchungsfeldes ein Datenvolumen von 1 bis 2 GB aufweisen. Im Kontrast hierzu stehen die konventionellen digitalen Aufnahmemethoden wie dem OPG oder FRS, die mit 1 MB pro Aufnahme lediglich 0,1 \% des Datenvolumens eines DVT-DICOM-Datensatzes einnehmen. Hierdurch entsteht eine zeitaufwendigere Befundung von DVT-Aufnahmen. Moderne digitale Volumentomographen bieten die Möglichkeit den zu untersuchenden Bereich vor dem Röntgenvorgang einzuschränken. Somit kann neben dem Datenvolumen auch die Strahlenbelastung reduziert werden (Ludlow et al. 2006). Außerdem besteht die Möglichkeit mit Hilfe einer Software zweidimensionale Übersichtsaufnahmen zu errechnen. Diese ähneln in ihrer Darstellung dem konventionellen OPG. Jedoch weisen sie keine der für das OPG charakteristischen Überlagerungen auf, da lediglich eine abgegrenzte Ebene abgebildet wird. Pathologische Prozesse außerhalb dieser Ebene könnten dem Untersucher somit verborgen bleiben.

Indikationseinschränkungen für die DVT entstehen durch eine im Vergleich zu konventionellen Aufnahmemethoden wie das OPG und FRS erhöhte Strahlenbelastung. Dosimetrische Untersuchungen an Kopf-Hals-Phantommodellen ergaben für hochauflösende DVTAufnahmen eine Strahlendosis von 131,7 bis $134,2 \mu$ Sv. Bei einer OPG-Aufnahme konnte eine Strahlendosis von 21,5 bis $21,87 \mu$ Sv gemessen werden, während FRS-Aufnahmen mit 4,5 bis 5,03 $\mu$ Sv die geringsten Strahlendosen unter den drei Aufnahmemethoden erzeugten (Grünheid et al. 2012; Signorelli et al. 2016). Bildartefakte bei metallischen Restaurati- 
onen und die fehlende Kostenübernahme durch die gesetzliche Krankenversicherung können die Indikation ebenfalls einschränken (Schulze et al. 2011). Aktuell werden FRS und OPG als Basisdiagnostik nicht von der DVT ersetzt (Kapila et al. 2011), sodass die Indikation einer solchen Aufnahme bei Fragestellungen gestellt wird, die nicht zufriedenstellend durch zweidimensionale Röntgenverfahren beantwortet werden können (Kaeppler und Mast 2012). Hierzu gehört zum Beispiel die röntgenologische Evaluation vestibulärer und lingualer Knochendefekte in der Parodontologie, die durch konventionelle Einzelzahnaufnahmen und das OPG nicht oder nur unzureichend erfolgen kann (Misch et al. 2006). Die Frage ob eine solche Erkenntnis zu einer besseren Therapie führt, ist nicht abschließend geklärt (Woelber et al. 2018). Der International Congress of Oral Implantologists veröffentlichte 2012 einen Konsensus Report bezüglich der Anwendung der DVT bei implantologischen Fragestellungen (Benavides et al. 2012). Dieses Review unterstützt die Anwendung der DVT bei Implantationsplanungen im Hinblick auf die Möglichkeit lineare Messungen in allen Dimensionen vorzunehmen und eine dreidimensionale Beurteilung der Anatomie des Alveolarkammes durchzuführen. Dennoch wird dazu geraten, die Vorteile und Risiken stets gegeneinander abzuwägen. Das Untersuchungsfeld sollte nicht größer als notwendig sein und vollständig befundet werden.

Abschließend kann festgehalten werden, dass die DVT Informationen bereitstellt, die durch konventionelle Röntgentechniken nicht erlangt werden. Ob diese Informationen die Therapieentscheidung maßgeblich positiv beeinflussen und die zusätzlichen Strahlenexpositionen und Kosten rechtfertigen, ist vom Behandler jeweils sorgfältig zu überprüfen.

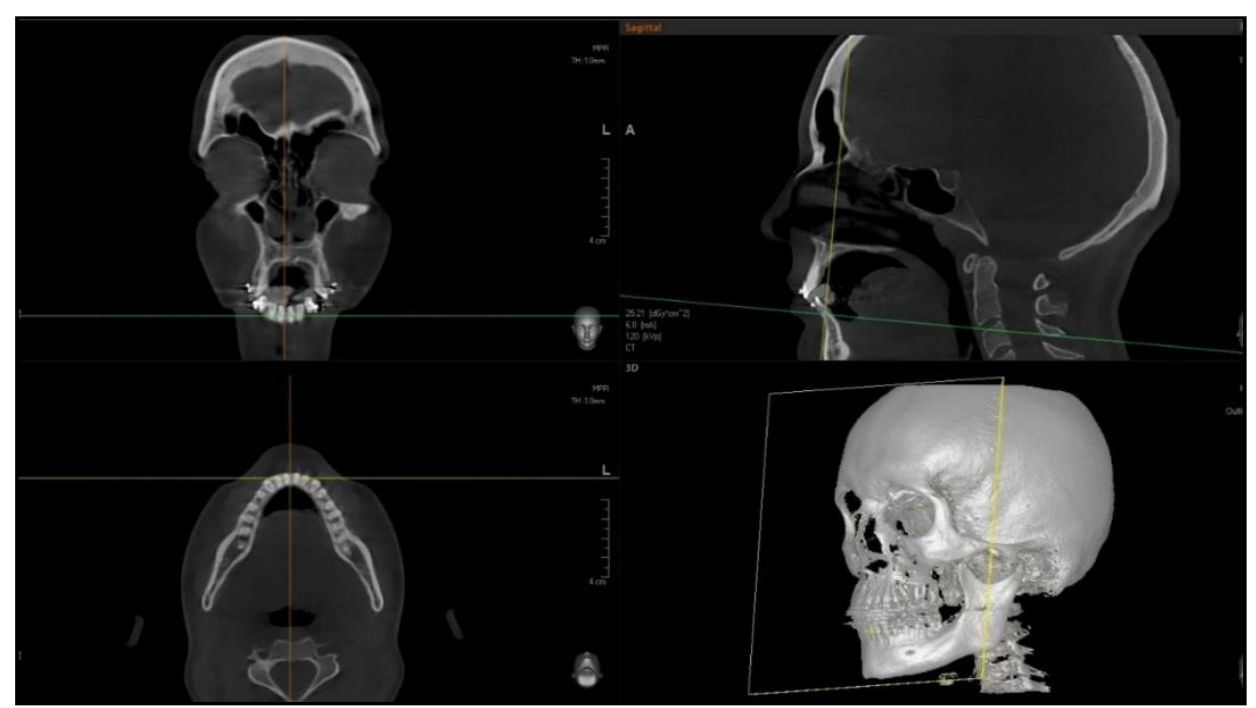

Softwaregestützte Darstellung der mittels DVT gewonnenen Daten in drei Ebenen mit zusätzlichem virtuellem 3D-Modell: Koronalebene (oben links), Sagittalebene (oben rechts), Axialebene (unten links), virtuelles 3D-Modell (unten rechts) (Klinik für Mund-, Kiefer- und Gesichtschirurgie Universitätsklinikum Göttingen) 
1.3 Röntgendiagnostik in Deutschland

In der Bundesrepublik Deutschland werden Röntgenuntersuchungen von dem Bundesamt für Strahlenschutz in jährlichen Statistiken erfasst und veröffentlicht (Bundesamt für Strahlenschutz - Röntgen - Röntgendiagnostik: Häufigkeit und Strahlenexposition).
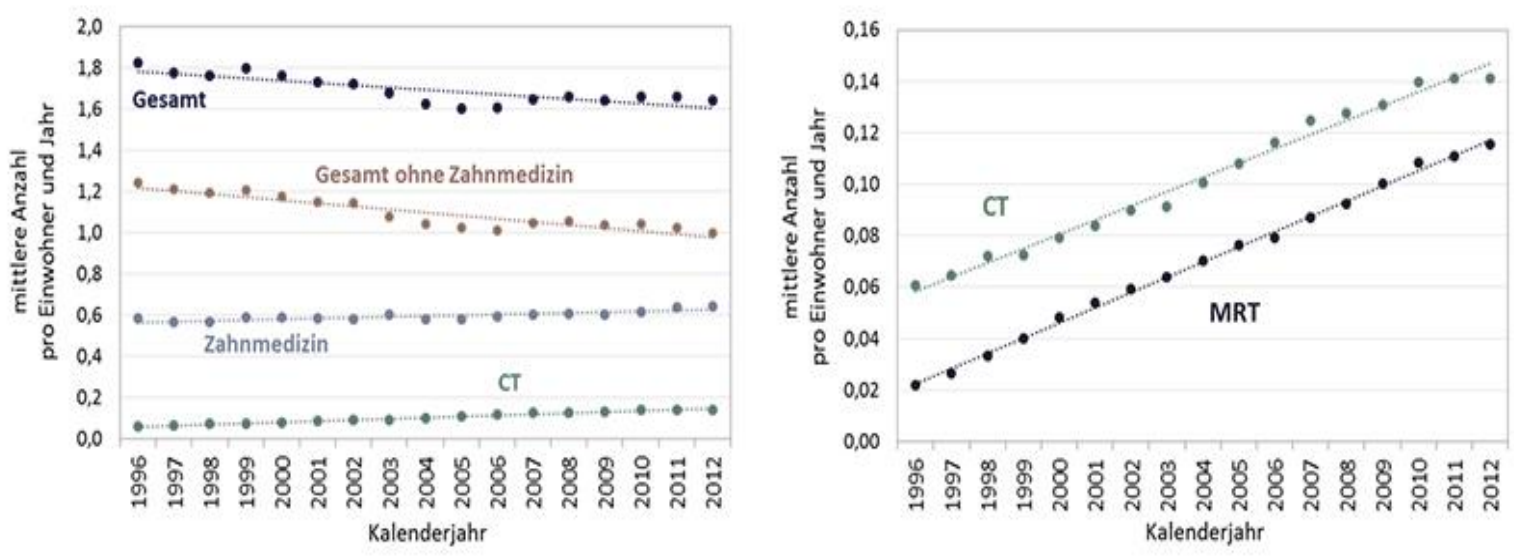

Abbildung 4 Anzahl der Röntgenuntersuchungen pro Einwohner und Jahr von 1996 bis 2012 (Bundesamt für Strahlenschutz, Salzgitter 2012)

Über jede dritte Röntgenaufnahme seit 1996 entsteht in der Zahnmedizin. Relevanter im Kontext des Strahlenschutzes ist jedoch die Strahlendosis, die als kollektive Dosis für das Jahr 2012 bestimmt wurde.
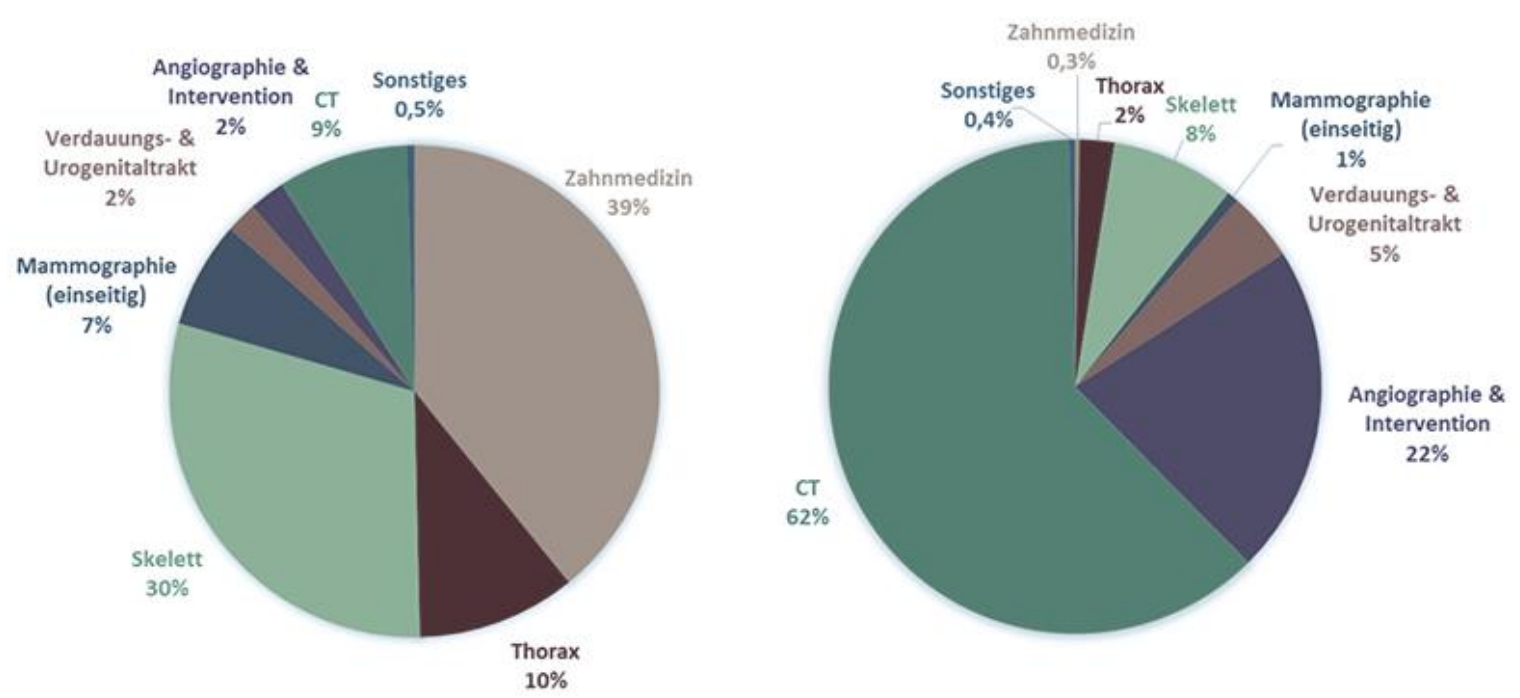
Prozentualer Anteil an der kollektiven Dosis (rechts) (Bundesamt für Strahlenschutz, Salzgitter 2012) 
Während die Zahnmedizin mit 39 \% eine Spitzenposition bei der Gesamthäufigkeit einnimmt, verursacht sie mit 0,3\% einen sehr geringen Anteil der kollektiven effektiven Dosis. Dies bedeutet, dass die Röntgendiagnostik in der Zahnmedizin relativ strahlungsarm ist. Im Kontrast hierzu stehen die strahlungsintensiveren CT-Aufnahmen und Angiographien nichtzahnmedizinischer Disziplinen, die 11 \% der Gesamthäufigkeit aber zugleich 84 \% der kollektiven effektiven Dosis ausmachen.

Trotz des sehr geringen Beitrages der Zahnmedizin an der kollektiven Strahlendosis birgt jede Strahlenexposition das Risiko maligner Entartungen, das in einem linearen Zusammenhang zu der Strahlendosis steht (Ludlow et al. 2008; Samartzis et al. 2011; Samartzis et al. 2013). Hieraus ergibt sich die Notwendigkeit der rechtfertigenden Indikation für radiologische Aufnahmen, die durch sorgfältige Abwägung von diagnostischem Nutzen und dem potentiellen Schaden durch Strahlenexposition entsteht (Ludlow et al. 2008; Katheria et al. 2010). Die Indikationsstellung kann durch eine verringerte Strahlendosis oder einen erhöhten diagnostischen Wert erleichtert werden, sodass die ideale Aufnahmetechnik ein maximales Volumen an wertvollen Informationen bei minimaler Strahlenbelastung aufweisen müsste. Diese kann beispielsweise bei dem FRS durch die Verwendung digitaler anstelle von konventionellen Röntgentechnologien halbiert werden (Visser et al. 2001). Die Erfahrung und das Wissen des diagnostizierenden Arztes ist ebenfalls ein wichtiger Faktor, da somit eine effizientere Auswertung der erlangten Daten ermöglicht wird (Omoumi et al. 2017; Bogaerts et al. 2018).

Weiteres Wissen bei der Befundung zweidimensionaler Röntgenaufnahmen könnte die Anwendungshäufigkeit zusätzlicher dreidimensionaler Aufnahmemethoden und eine weitere Strahlenbelastung reduzieren. Die radiologische Evaluation verfügbarer Knochenvolumina häufig genutzter intraoraler Spenderregionen für den Knochenaufbau in der dentalen Implantologie wird mit Hilfe von dreidimensionalen Röntgenaufnahmemethoden durchgeführt. Reproduzierbare Messungen an OPG- und FRS-Aufnahmen könnten Korrelationen zu diesen Knochenvolumina aufweisen. Die Kenntnis über solche Korrelationen könnte eine zielgerichtete Diagnostik von kleineren Untersuchungsfenstern und folglich eine Reduktion der Strahlenexposition ermöglichen. Aufgabe dieser Arbeit war es, OPG-, DVT- und FRSAufnahmen hinsichtlich solcher Korrelationen zu untersuchen. 


\subsection{Präimplantologische Knochenaugmentationen}

Die Versorgung eines lückigen Gebisses oder zahnlosen Patienten mit implantatgetragenem Zahnersatz setzt ein suffizientes Knochenangebot mit entsprechender Knochenhöhe, -breite und -dichte voraus. Dies ist eine der Voraussetzungen für die Osseointegration eines Implantates (Brånemark et al. 1977). Innerhalb des ersten Jahres nach Zahnverlust kommt es allerdings zu einer deutlichen Reduktion des Knochenangebotes in der betroffenen Region (Schropp et al. 2003), wobei das resultierende Minderangebot im koronalen Drittel der Alveole und auf der vestibulären Hälfte des Alveolarkammes besonders ausgeprägt ist (Araújo und Lindhe 2009). In der Molarenregion ist dies deutlicher (Araújo et al. 2015). Falls der Patient in diesen Fällen dennoch eine Implantation wünscht, ist eine vorangehende Augmentation des Knochenlagers unverzichtbar.

\subsubsection{Knochenaugmentationsmaterialien}

Während Transplantate für Knochenaugmentationen von verschiedenen Quellen bezogen werden können, sind drei unterschiedliche regenerative Mechanismen an erfolgreichen Knochenaugmentationen beteiligt (Garg und Terheyden 2006).

Durch die Osteokonduktion wird den umliegenden Zellen und Blutgefäßen ein passives Grundgerüst geboten. Ausgehend vom ortsständigen, vitalen Knochen kann durch Angiogenese die Migration von knochenbildenden Zellen und Nährstoffen erfolgen (AichelmannReidy und Yukna 1998; Nasr et al. 1999; Garg und Terheyden 2006). An den Defektgrenzen wird so das Knochenwachstum ermöglicht.

Bei der Osteoinduktion hingegen regen u. a. BMPs (Bone Morphogenic Proteins) eine Differenzierung pluripotenter mesenchymaler Stammzellen des Emfpängers zu Osteoblasten an, die anschließend neues Knochengewebe bilden können (Aichelmann-Reidy und Yukna 1998; Nasr et al. 1999; Albrektsson und Johansson 2001; Garg und Terheyden 2006). Somit handelt es sich bei der Osteoinduktion um einen Prozess, der im Gegensatz zur Osteokonduktion unabhängig von bereits differenzierten Knochenzellen ablaufen kann. 
Die Osteogenese beschreibt als letzter der drei Mechanismen einen Prozess, bei dem die direkte Bildung von Knochen durch z. B. transplantierte Osteoblasten erfolgt. Dies setzt voraus, dass die transplantierten Osteoblasten überleben, um eine vom Transplantat ausgehende Knochenneubildung zu erwirken. (Delloye et al. 2003).

Sämtliche Knochenaugmentationsmaterialien nutzen mindestens einen dieser Mechanismen (Garg und Terheyden 2006).

Es wird generell zwischen fünf Quellen für die Rekonstruktion von Geweben unterschieden. Alloplastische Materialien sind synthetischen Ursprungs. Diese organischen Materialen, Metalle oder Keramiken bieten durch ihre osteokonduktiven Eigenschaften ein passives Grundgerüst für umliegende Zellen und Blutgefäße (Aichelmann-Reidy und Yukna 1998; Nasr et al. 1999; Strietzel et al. 2007). Während die Verfügbarkeit Patientenunabhängig ist, bleibt die Anwendung mit Beschaffungskosten verbunden.

Dies trifft auch für xenogene Materialien zu. Allerdings stammen sie von Lebewesen, die nicht der gleichen Spezies angehören wie der Empfänger. Zumeist handelt es sich um das Rind (bovin), Pferd (equin) oder Schwein (porcin). Der Herstellungsprozess erlaubt durch Entfernung organischer Bestandteile die selektive Transplantation anorganischer Knochenbestandteile. Dadurch werden unerwünschte Immunreaktionen und das Infektionsrisiko erheblich reduziert (Taylor et al. 1996; Sogal und Tofe 1999; Wenz et al. 2001; Grobben et al. 2004). Eine Entfernung der antigenen Proteine bei hohen Temperaturen (>300 ${ }^{\circ} \mathrm{C}$ ) hat eine Reduktion der BMP-Aktivität und somit eine Verminderung bis Eliminierung osteoinduktiver Eigenschaften zur Folge (Garg und Terheyden 2006). Der Marktführer in Deutschland Bio-Oss ${ }^{\circledR}$ (Geistlich Pharma, Wolhusen, Schweiz) ist ein Beispiel hierfür. Jedoch existieren Untersuchungen, die osteoinduktive Prozesse auch bei thermisch behandelten deproteinierten xenogenen Knochenersatzmaterialien nachweisen konnten (Schwartz et al. 2000).

Stammt ein Knochenaugmentationsmaterial von einem anderen lebenden oder toten Individuum der gleichen Spezies, spricht man von allogenen Transplantaten. Spender und Empfänger müssen nicht histokompatibel sein, da allogene Materialien ebenfalls in einem Herstellungsprozess entsprechend aufbereitet werden. Durch die Gefrierung, Gefriertrocknung oder eine Kombination aus Gefriertrocknung und (Teil-)Demineralisation entstehen DFDBA/M Präparate (demineralized freeze-dried bone allograft oder matrix). Ein weiterer 
Typ ist der autolysierte, antigen-extrahierte allogene Knochen (AAA) (Kübler et al. 1999). Neben der Antigenität wird hierdurch das Infektionsrisiko durch beispielsweise das Humane Immundefizienz-Virus (HIV) oder Hepatitis Viren reduziert, aber nicht gänzlich eliminiert (Buck et al. 1989; von Garrel und Gotzen 1998).

Isogene Materialien stammen ebenfalls von einem anderen menschlichen Individuum. Bei dieser Sonderform des allogenen Transplantates handelt es sich bei Spender und Empfänger um eineiige Zwillinge und somit genetisch identische Individuen. Eine antigen-antikörperbedingte Abstoßung des Transplantates ist daher nicht zu erwarten (Gong et al. 2007).

Abschließend sind die autogenen Transplantate zu erwähnen, bei denen Spender und Empfänger identisch sind. Während die Antigenität kein Problem darstellt, muss der Behandler die Entnahmemorbidität sowie damit verbundene potentielle Komplikationen und verlängerte Eingriffszeiten bedenken (Weibull et al. 2009; Rogers und Greene 2012; Andersen et al. 2014; Varol et al. 2016). Neben osteokonduktiven und -induktiven Eigenschaften sind autogene Transplantate auch osteogen. Eine Übertragung von Infektionserkrankungen durch das Transplantat ist ausgeschlossen, während die Biokompatibilität optimal ist. Diese Eigenschaften machen sie zum „Goldstandard“ bei Kieferaugmentationen (Nasr et al. 1999; Hahn et al. 2008a; Rogers und Greene 2012).

Autogene Transplantate können intra- und extraoral gewonnen werden. Ein entscheidender Faktor bei der Auswahl ist die Verfügbarkeit von ausreichendem Knochenvolumen an dem Entnahmeort für die geplante Augmentation. Da es in der vorliegenden Arbeit um die Bewertung der Volumina intraoraler Knochentransplantate geht, sollen im Anschluss die verschiedenen intraoralen Spenderregionen beschrieben werden.

\subsubsection{Intraorale Spenderregionen autogener Knochentransplantate}

Für die Gewinnung von Knochentransplantaten zum präimplantologischen Alveolarkammaufbau lassen sich intra- von extraoralen Spenderregionen unterscheiden. Zu den Extraoralen gehören die Crista iliaca, Tibia, Fibula und Schädelkalotte. Diese finden vornehmlich Anwendung in Fällen, bei denen sich das intraorale Knochenangebot für den Aufbau größerer knöcherner Defekte als unzureichend erweist (Hahn et al. 2008b). Sie sind jedoch mit 
einem erhöhten Zeit- und Arbeitsaufwand bei zusätzlicher Entnahmemorbidität verbunden und können, zum Beispiel im Falle der Crista iliaca, neben einer Intubationsnarkose einen postoperativen Krankenhausaufenthalt des Patienten erfordern (Nkenke und Neukam 2014). Aus diesen Gründen weichen sie häufig den intraoralen Alternativen (Abb. 6). Neben den drei Entnahmeregionen, die in dieser Arbeit untersucht wurden, können im Oberkiefer die Spina nasalis und der Tuber maxillae für die Knochengewinnung genutzt werden. Im Unterkiefer steht zudem noch die laterale Kortikalis zur Verfügung (Hahn et al. 2008b).

Im Bereich der Unterkiefersymphyse besteht in der Regel das größte intraorale Knochenangebot (Zeltner et al. 2016). Hier ist es möglich neben Kompaktablöcken auch größere Mengen Spongiosa zu entnehmen (Pikos 1995). Es ist zu beachten, dass sich in der unmittelbaren Umgebung das paarige Foramen mentale und die Wurzelspitzen der Unterkiefer Incisivi und Eckzähne befinden. Zudem darf die linguale Kortikalis nicht beschädigt werden, da Gefäß- und Weichgewebeläsionen zu starken Blutungen und einer Verlegung der Atemwege führen könnten. Diese Strukturen sind während der Knochenentnahme unbedingt zu schonen um irreversible Schäden zu vermeiden. Trotz des relativ großen verfügbaren Knochenvolumens wird die Symphyse von einigen Autoren nicht als die erste Wahl angesehen. Dies wird mit vergleichsweise häufigeren und stärker ausgeprägten postoperativen Beschwerden als bei anderen intraoralen Spenderregionen begründet. Zu diesen gehören neben Sensibilitätsstörungen der Unterkiefer Incisivi und des Kinns auch das Tropfenkinn (Clavero und Lundgren 2003; Nkenke und Neukam 2014; Reininger et al. 2016).

Eine geringere Entnahmemorbidität bei kleinerem, verfügbarem Knochenvolumen als die Kinnregion weist der paarige Ramus mandibulae samt Retromolarregion auf (Clavero und Lundgren 2003; Silva et al. 2006; Weibull et al. 2009; Nkenke und Neukam 2014). Aufgrund der vorwiegend kortikalen Struktur ist mit einer nur geringen Resorption des Transplantates zu rechnen (Misch 2000). Monokortikale Knochenblocktransplantate weisen vereinzelte, transient avitale Bereiche auf, die als Folge der Revaskularisation nach einer Dauer von ca. 7 Monaten wieder mit Osteozyten besiedelt werden (Zerbo et al. 2003; Acocella et al. 2010). In der Retromolarregion gilt es den Nervus alveolaris inferior, Nervus lingualis und distalsten Zahn zu schonen. Der linguale Kortex ist zu belassen, um das Risiko einer Läsion des Nervus lingualis zu vermindern. 
Bei der Crista zygomatico-alveolaris handelt es sich um die kleinste der drei untersuchten intraoralen Spenderregionen (Zeltner et al. 2016). Sie ist meist im Bereich des ersten Oberkiefermolaren lokalisiert und liegt im Übergang der Maxilla zum Os zygomaticum. Da sie die laterale Wand des Sinus maxillaris darstellt, ist bei einer Entnahme das Erhalten der Schneider-Membran anzustreben. Hierfür kann die Anwendung piezochirurgischer Instrumente hilfreich sein (Vercellotti et al. 2001; Stübinger et al. 2006).

Aus den beschriebenen intraoralen Spenderregionen können deutlich unterschiedliche Knochenvolumina gewonnen werden. Diese Differenzen entstehen durch anatomisch-morphologische Eigenschaften und begrenzende, zu schonende Nachbarstrukturen in der jeweiligen Region (Zeltner et al. 2016). Zudem zeigt die klinische Erfahrung, dass interindividuelle Unterschiede bezüglich des Knochenangebotes in derselben Region bestehen (Yates et al. 2013). Das Auffinden einer jeweils geeigneten Spenderregion stellt somit eine zentrale Aufgabe für den Chirurgen dar, die für jeden Patienten individuelle Lösungen erfordert.

Diese Arbeit soll bei der Suche nach einer geeigneten Region eine Hilfestellung leisten. Hierzu wird nach Korrelationen zwischen kephalometrischen Messwerten, wie sie in der Kieferorthopädie üblich sind und Knochenvolumina sowie -dimensionen gesucht. Kenntnisse über solche Zusammenhänge könnten bei der initialen Patientenberatung und gezielten, weiter eingegrenzten Suche mit DVT-Aufnahmen hilfreich sein.

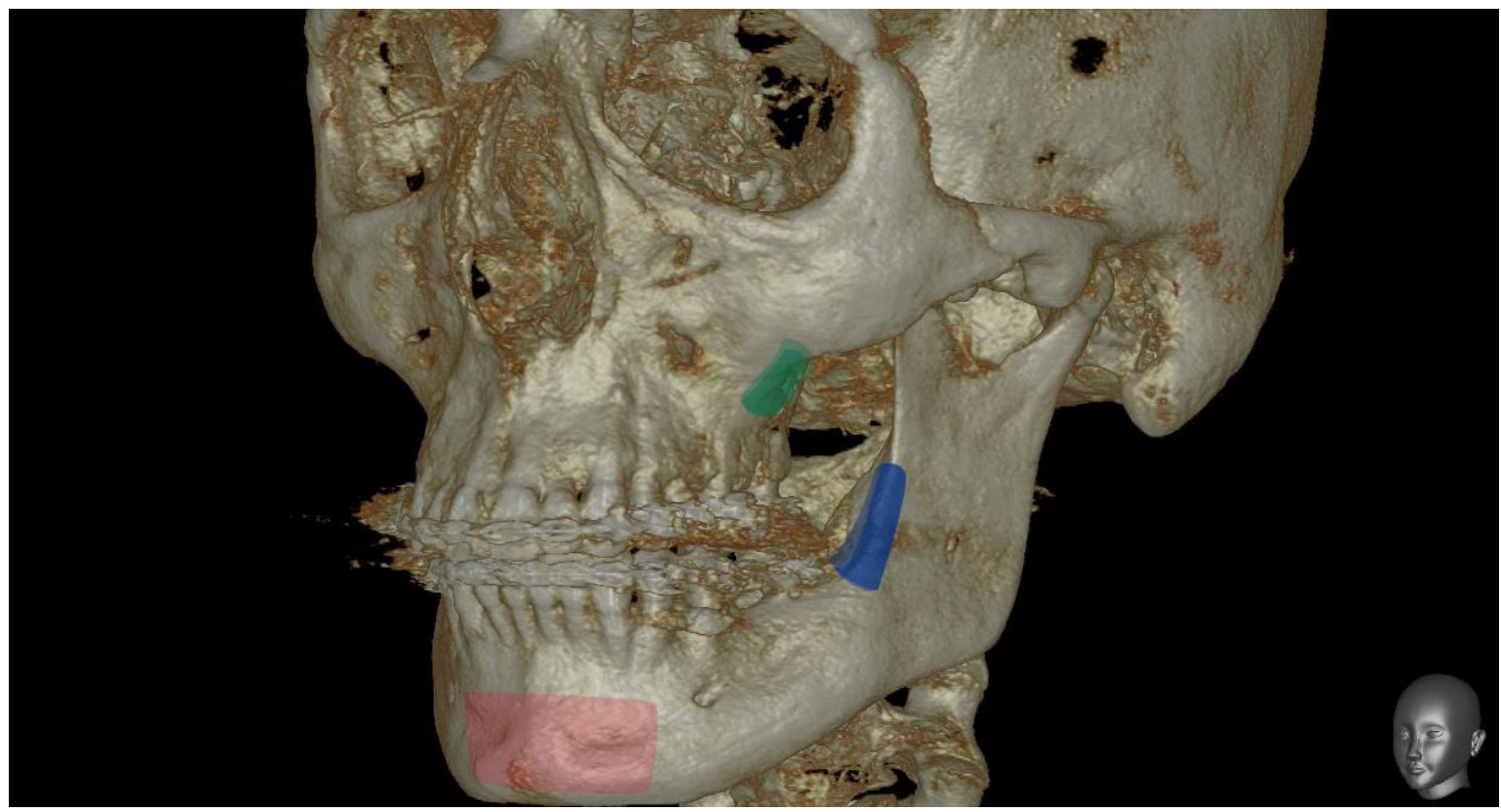
gionen: Unterkiefersymphyse (rot), Retromolarregion \& Ramus mandibulae (blau), Crista zygomatico-alveolaris (grün) (Klinik für Mund-, Kiefer- und Gesichtschirurgie Universitätsklinikum Göttingen) 
1.5 Volumetrische Untersuchungen am menschlichen Schädel

Zu volumetrischen Messungen am menschlichen Schädel liegt bereits eine Vielzahl an Veröffentlichungen vor (Yavuz et al. 2009; Katayama et al. 2014; Zeltner et al. 2016). Radiologische Messungen wurden entweder an klinischen Aufnahmen oder humanen Schädelpräparaten durchgeführt, während humane Schädelpräparate zusätzlich für manuelle Messungen herangezogen wurden. Kombinationen beider Methoden dienten einer Evaluation der Genauigkeit von softwaregestützten Messungen in Röntgenaufnahmen. Cavalcanti et al. (2004), Liu et al. (2006) und Berco et al. (2009) nahmen erst lineare Messungen an dreidimensionalen Röntgenaufnahmen (CT oder DVT) humaner Schädelpräparate vor und verglichen diese Messergebnisse mit manuellen Messungen an den entsprechenden Schädeln. Hierbei konnten keine statistisch signifikanten Unterschiede zwischen den manuellen Messungen an humanen Schädelpräparaten und den Messungen an Röntgenaufnahmen festgestellt werden.

Zeltner et al. (2016) richteten ihr Augenmerk auf die Knochenvolumina potentieller Spenderregionen im Bereich der Kinnsymphyse und der retromolaren Areale der Mandibula. Diese Messungen an DVT-Aufnahmen ergaben für das Kinn mit durchschnittlich 3,5 $\pm 1,3$ $\mathrm{cm}^{3}$, fast doppelt so viel Knochenvolumen wie in der Retromolarregion mit durchschnittlich $1,8 \pm 1,1 \mathrm{~cm}^{3}$. Die Crista zygomatico-alveolaris war nicht Gegenstand dieser Studie. Yavuz et al. (2009) untersuchten in ihrer Arbeit ausschließlich das Knochenvolumen im Bereich der Kinnsymphyse und haben ein durchschnittliches Knochenvolumen von 3,49 $\pm 0,77 \mathrm{~cm}^{3}$ gemessen.

In zwei anderen Veröffentlichungen wurde das Knochenvolumen der gesamten Mandibula exklusive der Zähne untersucht (Deguchi et al. 2010; Katayama et al. 2014). Ziel dieser Studien war es, Korrelationen zwischen dem Gesamtknochenvolumen der Mandibula und kephalometrischen Messungen am FRS nachzuweisen. Drei skelettale Klassen, eingeteilt nach ANB, wiesen keine statistisch signifikanten Unterschiede bezüglich ihrer Korrelationen mit dem gesamten mandibulären Knochenvolumen auf (Deguchi et al. 2010; Katayama et al. 2014). Der im FRS bestimmte Kieferwinkel hingegen wies eine signifikante negative Korrelation mit dem Gesamtknochenvolumen der Mandibula auf (Katayama et al. 2014). Während in diesen Arbeiten nach Korrelationen zwischen kephalometrischen Messungen an 
zweidimensionalen Röntgenbildern und Knochenvolumina in DVT-Aufnahmen gesucht wurde, unterschied man nicht zwischen den einzelnen, gängigen intraoralen Knochenentnahmeregionen.

Bislang sind keine Untersuchungen zu Korrelationen zwischen den Knochenvolumina geläufiger Knochenspenderregionen und standardisierten, kephalometrischen Messungen vorhanden. In der vorliegenden Arbeit wird daher eine Differenzierung einzelner Knochenentnahmeregionen bei der Suche nach Korrelationen vorgenommen. Neben dem Knochenvolumen werden zusätzlich die drei Dimensionen der Knochenblöcke vermessen und ebenfalls auf Korrelationen mit kephalometrischen Parametern untersucht.

\subsection{Studienziel}

Das Ziel dieser Studie ist es, den diagnostischen Wert von zweidimensionalen bildgebenden Verfahren (OPG, FRS) im Kontext autologer Knochentransplantationen zu ermitteln. Dies soll durch einen Vergleich mit den dreidimensionalen bildgebenden Verfahren (DVT, CT) ermöglicht werden. Hierdurch sollen signifikante Korrelationen reproduzierbarer Messungen zwischen zwei- und dreidimensionalen Abbildungen überprüft werden. Diese könnten als hilfreiche Anhaltspunkte bei initialen Beratungsgesprächen mit Patienten fungieren und eine gezieltere Suche mit Hilfe der dreidimensionalen Bildgebung ermöglichen, um die Strahlenexposition und den Zeitaufwand zu reduzieren. 


\section{Material und Methoden}

Im folgenden Kapitel werden die angewandten Methoden erläutert, mit denen die Messungen an FRS-, DVT- und OPG-Aufnahmen durchgeführt wurden. Anschließend werden die statistischen Methoden dargestellt, die bei der Auswertung der gewonnenen zur Anwendung kamen.

\subsection{Patientenkollektiv}

Insgesamt wurden 108 Röntgenbilder von 36 Patienten untersucht, die im Rahmen von zahnärztlichen, kieferorthopädischen und Mund-, Kiefer-, Gesichtschirurgischen Behandlungen angefertigt wurden. Für die Auswertung lagen 36 DVT-, 36 OPG- und 36 FRS-Aufnahmen vor. Das Patientenalter innerhalb des Kollektivs, das aus 20 Frauen und 16 Männern besteht, reicht von 18 bis 51 Jahren. Voraussetzungen für die Aufnahme in die Untersuchung waren ein abgeschlossenes Knochenwachstum, sowie das Vorhandensein aller Stützzonen nach der Eichner Klassifikation bei natürlicher Bezahnung. Kraniofaziale Anomalien wie z. B. Lippen-Kiefer-Gaumenspalten, durchgeführte bimaxilläre Umstellungsosteotomien und knöcherne Traumata stellten ein Ausschlusskriterium dar. Pathologische Prozesse in potentiellen Knochenspenderregionen führten ebenfalls zum Ausschluss.

\subsection{Ethische Richtlinien}

Für diese Arbeit wurden keine Versuche an Menschen oder Tieren durchgeführt. Jegliche ausgewerteten Bilddaten lagen im Universitätsklinikum Göttingen bereits vor. Sie dürfen gemäß den allgemeinen Vertragsbedingungen des Klinikums analysiert werden, sofern sichergestellt ist, dass keine patientenbezogenen Daten an Personen außerhalb des Klinikums gelangen. Der Deklaration von Helsinki entsprechend zählt die Auswertung medizinischer Daten zu der medizinischen Forschung und muss daher einer Ethikkommission vorgelegt werden. Unter der Antragsnummer DOK_342_2015 wurde das Votum der Ethikkommission eingeholt und dem Forschungsvorhaben in der vorliegenden Form zugestimmt. 


\subsection{Untersuchungsmethoden}

\subsubsection{Analyse des Fernröntgenseitenbildes (FRS)}

Die kephalometrische Analyse der Fernröntgenseitenbilder erfolgte mit Hilfe der Therapieplanungssoftware ivoris ${ }^{\circledR}$ analyze (Computer konkret AG, Falkenstein, Deutschland). Zur Anwendung kam hierbei die modifizierte kephalometrische Analyse nach Hasund, wobei im Rahmen dieser Arbeit folgende Variablen im FRS bestimmt wurden:

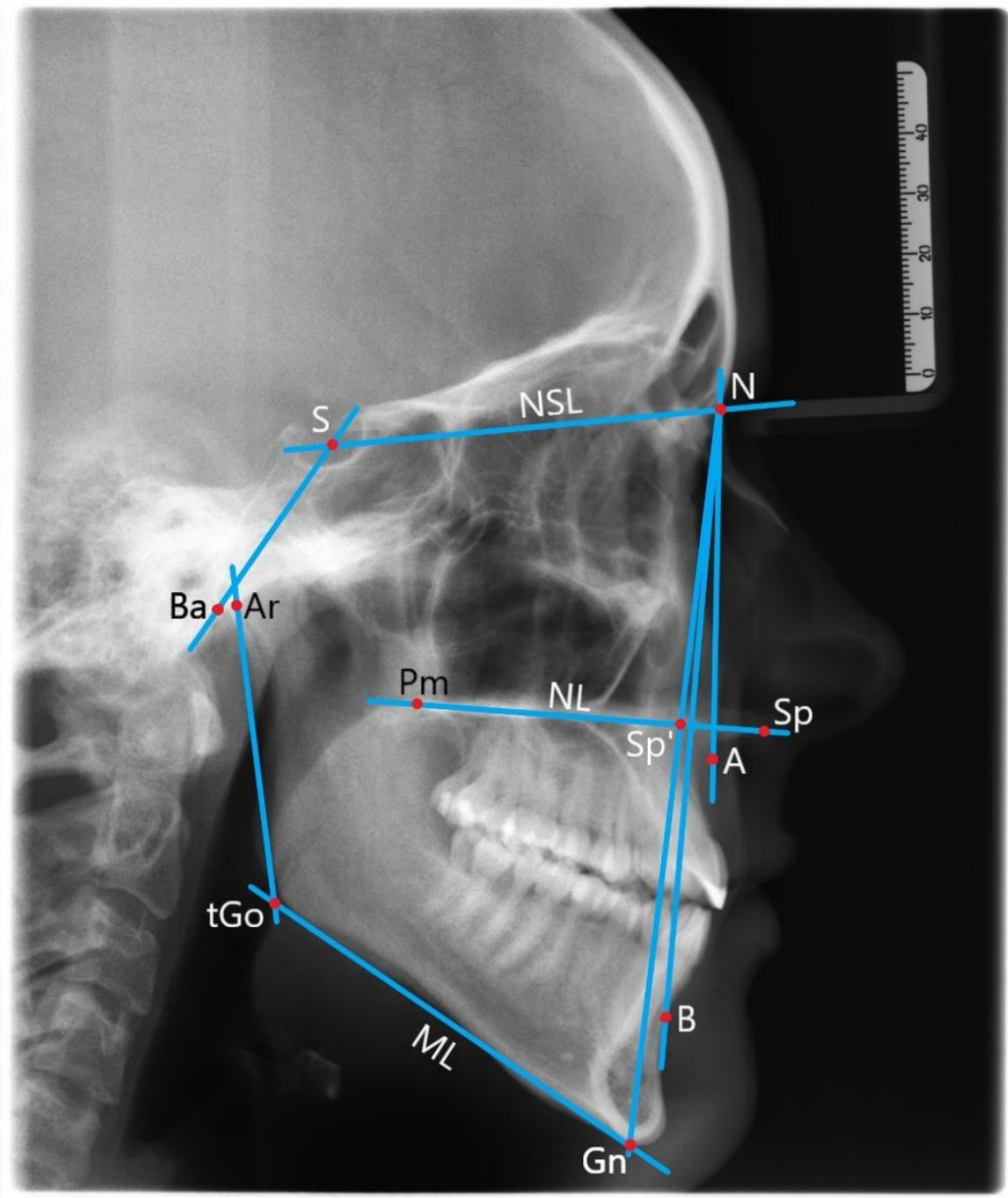

Abbildung 7

... 'FRS mit eingezeichneten kephalometrischen Variablen: SNA-Winkel, SNB-Winkel, ANB-Winkel, MLNSL-Winkel, NL-NSL-Winkel, ML-NL-Winkel, NSBa-Winkel, Gn-Go-Ar-Winkel, Index $=\frac{N-S p \prime}{S p^{\prime}-G n} \times 100$ (Poliklinik für Kieferorthopädie Universitätsklinikum Göttingen)

Zu Beginn der kephalometrischen Analyse wurden folgende Referenzpunkte manuell gekennzeichnet: Nasion (N), Sella (S), Basion (Ba), Articulare (Ar), Goniontangentenpunkt (tGo) Gnathion (Gn), A-Punkt (A), B-Punkt (B), Spina (Sp) und Pterygomandibulare (Pm). Nach Anlegen dieser, wurden sämtliche hieraus konstruierbaren Linien und Winkel durch die 
Software automatisch ergänzt und errechnet. Der SNA-Winkel beschreibt die sagittale Lage des A-Punktes, während der SNB-Winkel die sagittale Lage des B-Punktes relativ zur anterioren Schädelbasis (NSL) beschreibt. Aus der Differenz zwischen SNA- und SNB-Winkel, ergibt sich der ANB-Winkel, der die Lage der Maxilla relativ zur Mandibula beschreibt. Da die sagittale Position der Maxilla relativ zur Schädelbasis (SNA) und die Neigung der Mandibula (ML-NSL) den ANB beeinflussen und eine tatsächliche sagittale Kieferrelation verdecken können, besteht mit Hilfe der Formel (iANB $=-35,16+0,4 x S N A+0,2 x M L-N S L$ ) eine Möglichkeit diesen Einfluss zu nivellieren (Panagiotidis und Witt 2005). Um die Neigung der Mandibula (ML-NSL) relativ zur anterioren Schädelbasis zu beschreiben, wird ein Winkel aus der Mandibularlinie (ML) und der anterioren Schädelbasis (NSL) gebildet. Nutzt man anstelle der Mandibularlinie die Nasallinie (NL), wird hingegen die Neigung der Maxilla (NL-NSL) beschrieben. Der auch als Interbasiswinkel bezeichnete ML-NL wird durch die Mandibular- und Nasallinie gebildet und kann basal vertikale Verhältnisse darstellen. Die anteriore Schädelbasis (NS) bildet durch die Abknickung des posterior befindlichen Clivus den NSBa-Winkel. Ramus und Corpus mandibulae bilden den Kieferwinkel (Gn-Go-Ar).

Abschließend wurde die strukturelle Methode nach Björk am FRS angewandt, welche für die frühzeitige Evaluation extremer Wachstumsmuster verwendet wird (Björk 1969; Nielsen 2011; Lenza et al. 2015). Neben drei kephalometrischen Messwerten (PgNB, Index und Gn-tgo-Ar) werden hierzu morphologische Eigenschaften der Mandibula im FRS untersucht: Kondylenform, Verlauf des canalis mandibularis und der Unterrand der Mandibula (Abb. 8 und 9). Nach Abgleich mit einer Tabelle (Abb. 10) kann ein „Score“ ermittelt werden, der Aufschluss über das Wachstumsmuster geben kann. Dabei deutet eine negative Summe der sechs Parameter auf eine posteriore Rotationstendenz hin.

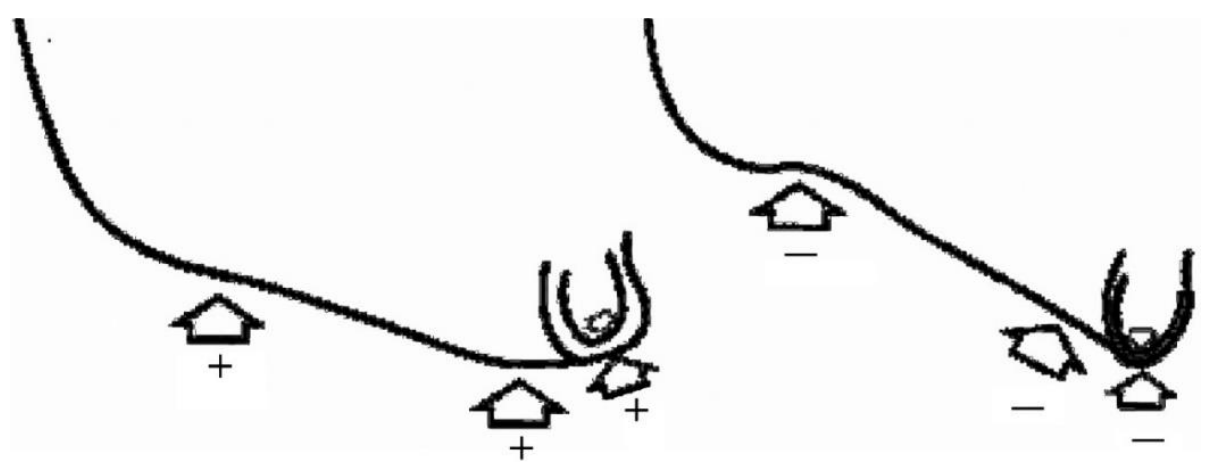

Abbildung $8 \quad$ Zu untersuchende morphologische Eigenschaften des mandibulären Unterrandes. Abbildung aus „Individualisierte Kephalometrie“ von Segner \& Hasund (Mit freundlicher Genehmigung durch Prof. Dr. Dietmar Segner, Hamburg) 


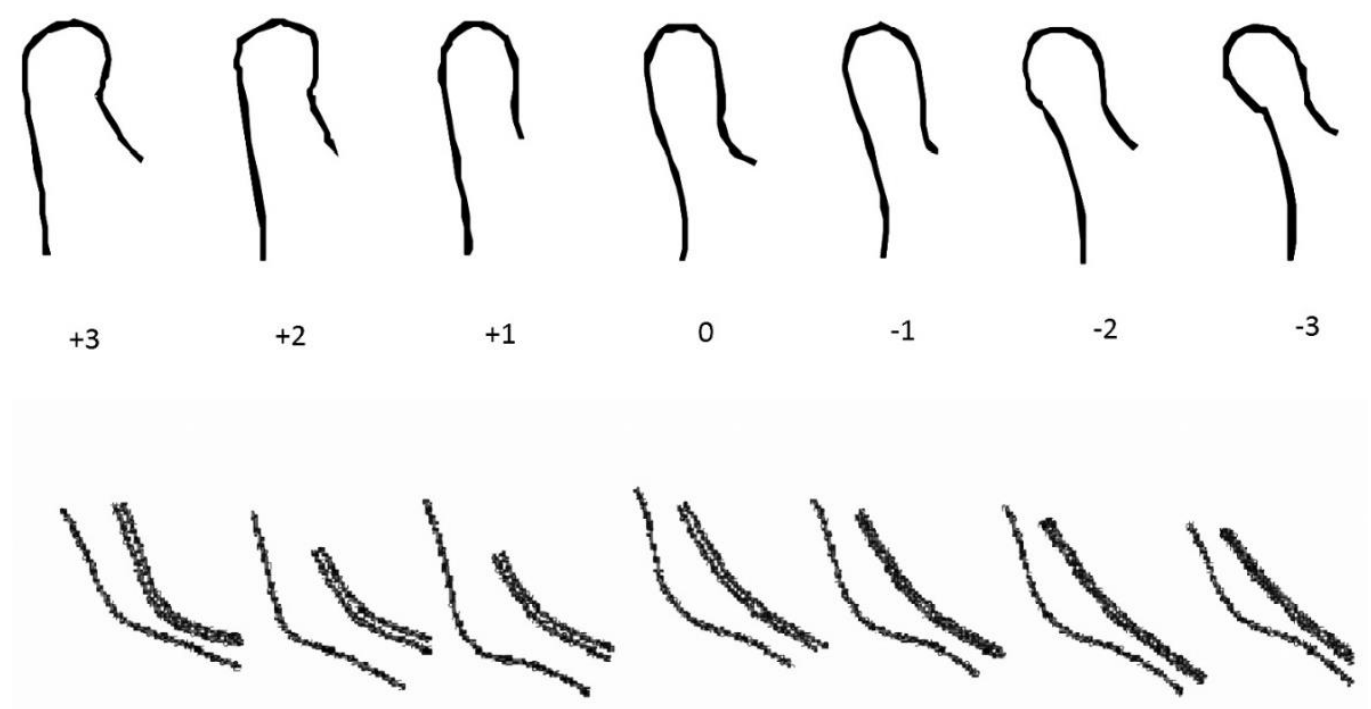

Abbildung 10 Form des Kondylus und canalis mandibularis mit entsprechendem „Score“. Abbildung aus „Individualisierte Kephalometrie" von Segner \& Hasund Hasund (Mit freundlicher Genehmigung durch Prof. Dr. Dietmar Segner, Hamburg)

\begin{tabular}{|c|c|c|c|}
\hline $\begin{array}{l}\text { Rotations- } \\
\text { tendenz }\end{array}$ & $\begin{array}{c}\text { Kinn- } \\
\text { prominenz } \\
\left(\mathrm{PgNB}_{\mathrm{mm}}\right)\end{array}$ & $\begin{array}{l}\text { Index } \\
\qquad \%\end{array}$ & $\begin{array}{c}\text { Kieferwinkel } \\
\text { (Gn-tgo-Ar) }\end{array}$ \\
\hline+++ & $>3.0$ & $>88$ & $<118$ \\
\hline++ & $2.0-3.0$ & $84-88$ & $118-123$ \\
\hline+ & $1.5-2.0$ & $80-84$ & $123-127$ \\
\hline 0 & $1.0-1.5$ & $75-80$ & $127-133$ \\
\hline- & $0.5-1.0$ & $71-75$ & $133-136$ \\
\hline- & $0.0-0.5$ & $66-71$ & $136-140$ \\
\hline$\ldots$ & $<0.0$ & $<66$ & $>140$ \\
\hline
\end{tabular}

Anhand dieser drei Grafiken und der Tabelle kann für jedes FRS eine Summe aus den einzelnen "Scores" ermittelt werden, die zwischen -18 und +18 liegt. Nach Segner und Hasund liegt der durchschnittliche Wert der sechs Merkmale bei +3 . Ist der Wert hingegen kleiner als -6, muss mit einer starken posterioren Rotation der Mandibula gerechnet werden. Ein Wert größer als +12 weist auf eine dominante anteriore Rotation hin (Segner und Hasund 1998). 


\subsubsection{Analyse der OPG-Aufnahmen}

Die Auswertung der OPG-Aufnahmen wurde mit Hilfe der Software SIDEXIS XG ${ }^{\circledR}$ (Firma Sirona Dental Systems GmbH, Bensheim, Deutschland) durchgeführt. Hierbei wurde der Abstand der Wurzelspitze des ersten Unterkiefer Schneidezahnes (WS1) und des zweiten Unterkiefer Molaren (WS7) zur Basis der Mandibula gemessen. Falls für die mittleren Incisivi unterschiedliche Streckenlängen vorlagen, wurde der Mittelwert erfasst. Dies gilt auch für die zwei Wurzelspitzen der Molaren, wobei hier zusätzlich der Mittelwert beider Seiten bestimmt wurde. Anschließend wurde der Quotient der beiden Strecken errechnet (WS1 / WS7) und der Kieferwinkel im OPG bestimmt (Ang. Mand.). Diese Messungen wurden auf Korrelationen mit den FRS- und DVT-Messwerten untersucht. Bei vorliegenden Korrelationen zu FRS-Messwerten könnten, anhand der OPG-Messungen, Aussagen zu Wachstumsmustern getroffen werden.

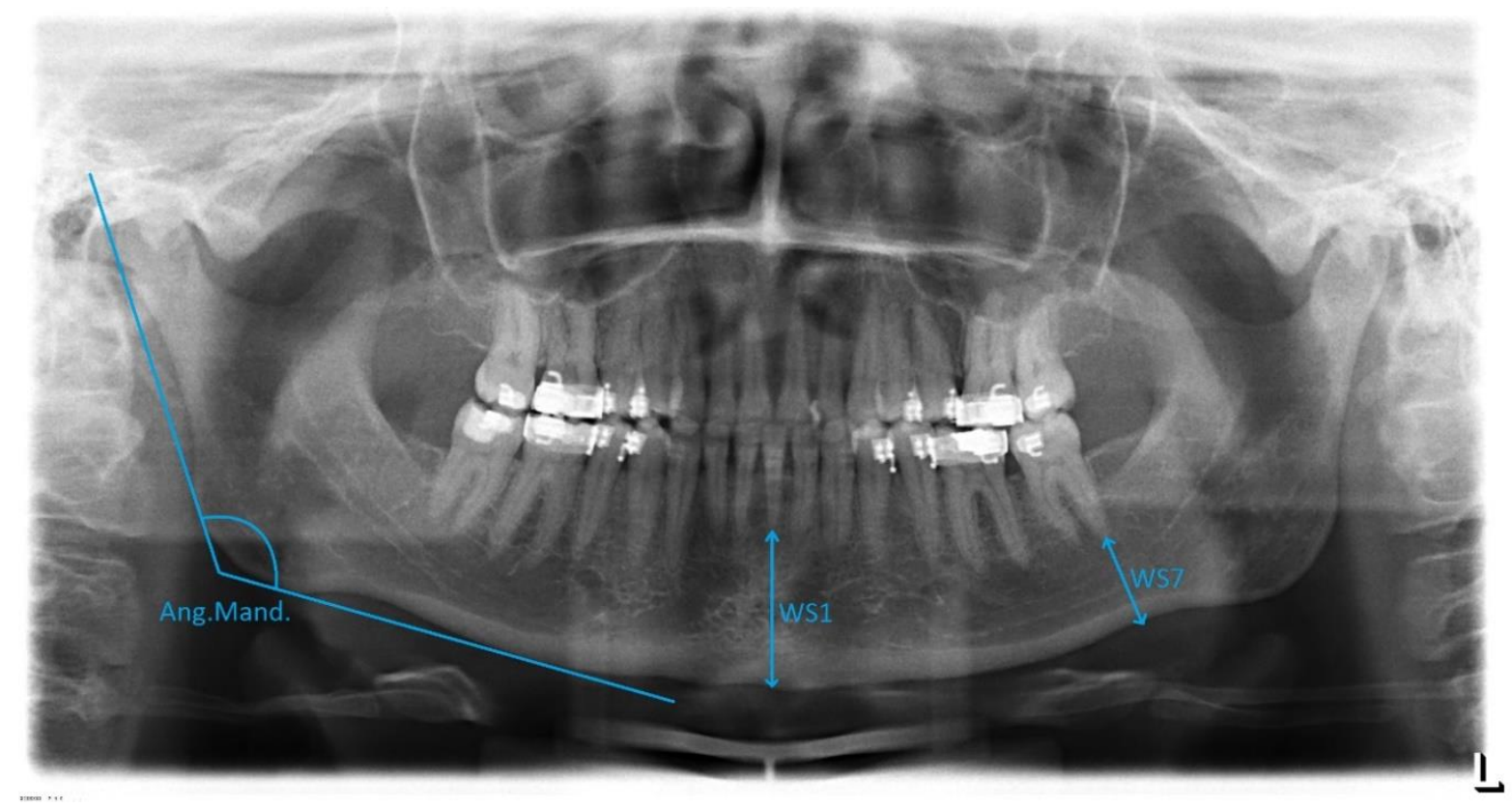

Abbildung 11

OPG mit Einzeichnungen der vorgenommenen Messungen. Abstand der Wurzelspitze des mittleren Schneidezahnes (WS1) und zweiten Molaren (WS7) zur Basis der Mandibula. Kieferwinkel (Ang. Mand.) (Poliklinik für Kieferorthopädie Universitätsklinikum Göttingen) 


\subsubsection{Analyse der DVT-Aufnahmen}

Die untersuchten DVT-DICOM Datensätze der Klinik für Mund-, Kiefer- und Gesichtschirurgie des Universitätsklinikums Göttingen wurden in die Analyse-Software Ez3D Plus ${ }^{\circledR}$ (Firma Vatech, Hwaseong, Korea) importiert. Bei jedem Patienten wurden drei potentielle Knochenspenderregionen untersucht: die Kinnregion, die paarigen retromolaren Areale inklusive der bukkalen Knochenlamelle der zweiten Unterkiefer-Molaren und die ebenfalls paarige Crista zygomatico-alveolaris. Im Falle der paarigen Strukturen wurden die Messungen als Mittelwerte notiert. Vor Durchführung der Messungen wurden angrenzende, zu schonende anatomische Strukturen mit einem Sicherheitsabstand gekennzeichnet (Zeltner et al. 2016). Im Falle des Kinns wurden 5 mm Abstand zu den Wurzelspitzen und dem Foramen mentale eingehalten (Abb. 12 und 13). Außerdem wurden $2 \mathrm{~mm}$ Abstand zu der lingualen und kaudalen Fläche der Mandibula gehalten (Abb. 13).

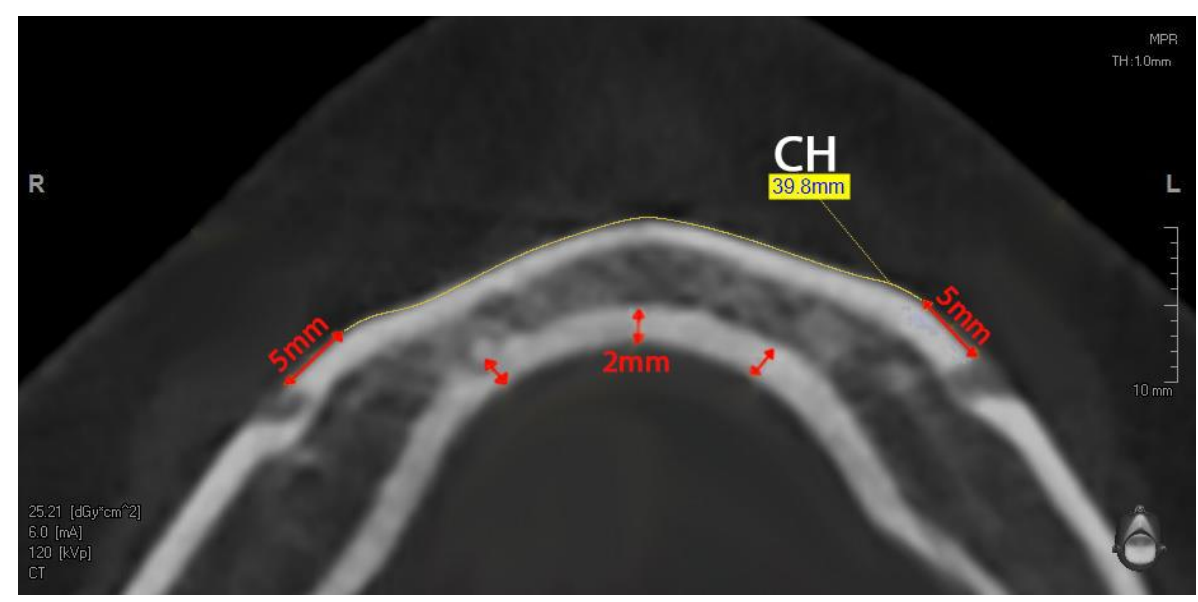
Messung $(\mathrm{CH})$

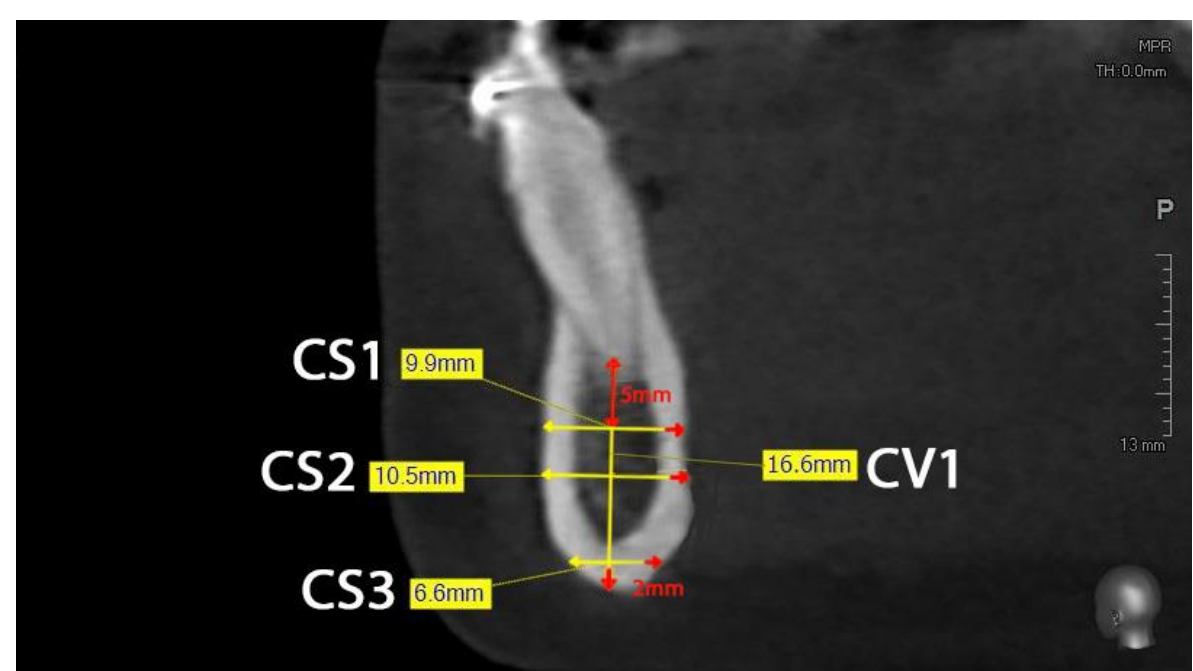


Anschließend wurde die Distanz zwischen den Wurzelspitzen der Eckzähne und der mittleren Incisivi zu der kaudalen Fläche der Mandibula gemessen (CV1 bis CV3, Abb. 13 und 14). Rechtwinklig zu diesen drei vertikalen Strecken, wurde an jeweils drei Punkten die Breite des Alveolarkammes gemessen (CS1 bis CS9, Abb. 14). Die kraniale Breitenmessung erfolgte im Abstand von $5 \mathrm{~mm}$ zu der jeweiligen Wurzelspitze, während die kaudalste bei einem $2 \mathrm{~mm}$ großen Abstand zur kaudalen Fläche der Mandibula stattfand. Zwischen diesen beiden erfolgte eine weitere Messung auf Höhe des Pogonions. Analog zu der Lage der Breitenmessungen (CS) wurde die horizontale Ausdehnung des potentiellen Transplantates ebenfalls an drei Stellen vermessen ( $\mathrm{CH} 1$ bis $\mathrm{CH} 3)$. Hierbei wurde ein $5 \mathrm{~mm}$ großer Sicherheitsabstand zum Foramen mentale bewahrt.

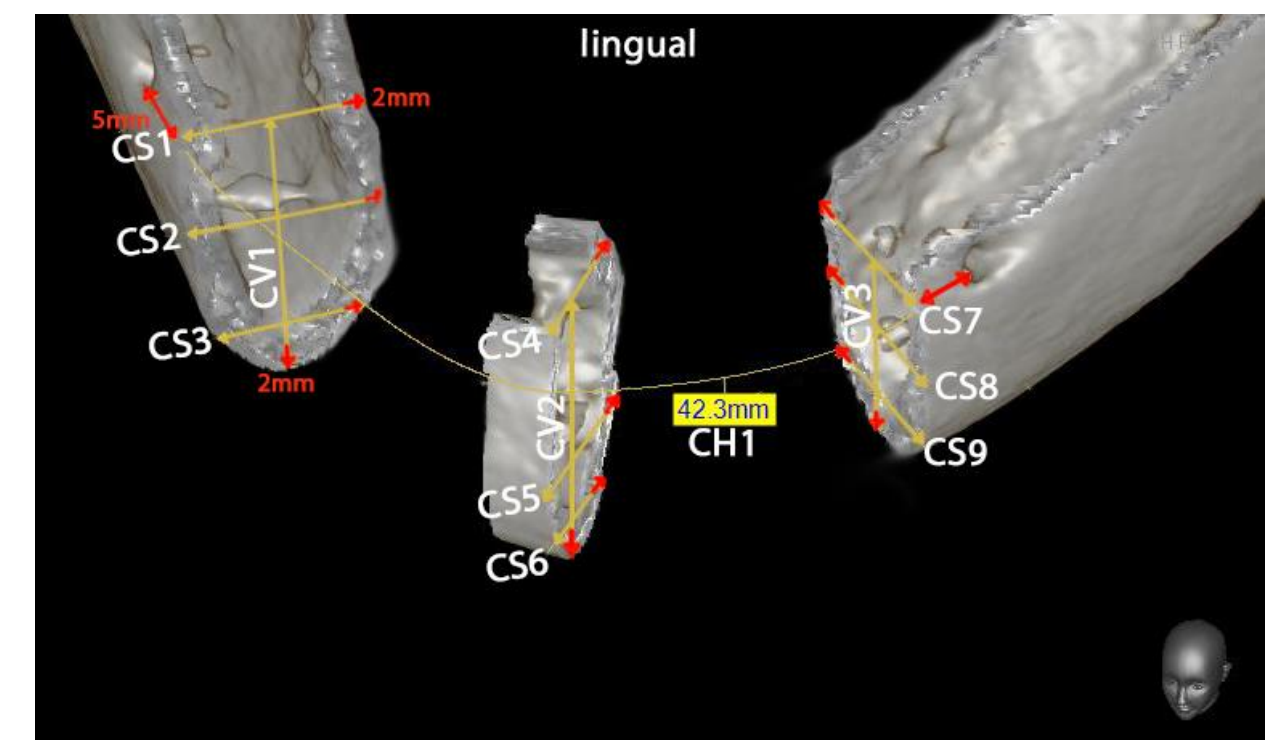
und Sicherheitsmarkierungen (rot) (Klinik für Mund-, Kiefer- und Gesichtschirurgie Universitätsklinikum Göttingen)

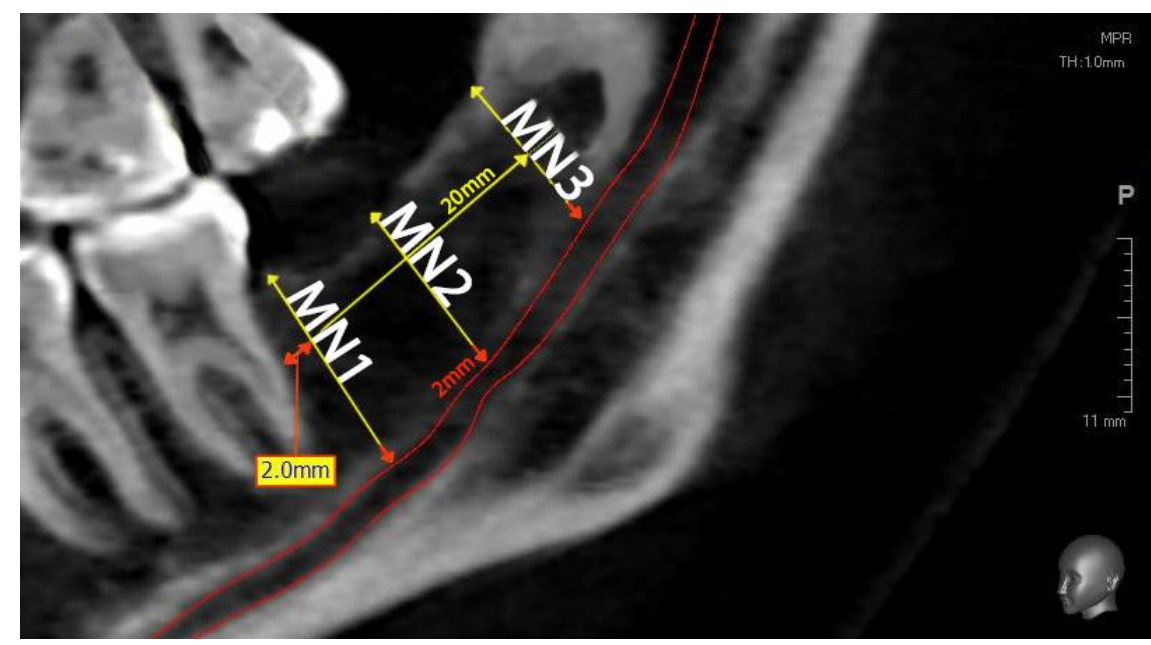
inferior und Sicherheitsabstand sowie Höhenmessungen des retromolaren Knochenblocks (MN) (Klinik für Mund-, Kiefer- und Gesichtschirurgie Universitätsklinikum Göttingen) 
Die retromolaren Knochenblöcke wurden mit einem Sicherheitsabstand von $2 \mathrm{~mm}$ zum farblich markierten Nervus alveolaris inferior und ebenfalls $2 \mathrm{~mm}$ zum distalsten Zahn vermessen (Abb. 15 und 16). Wie auch beim Kinn wurde eine $2 \mathrm{~mm}$ dicke Knochenschicht an der lingualen Fläche der Mandibula belassen (Zeltner et al. 2016). Die mesio-distale Länge des Knochenblockes wurde mit 20 mm festgelegt. Daraufhin wurde die vertikale Distanz des Nervus alveolaris inferior zu der okklusal gelegenen Knochenoberfläche an drei Punkten bestimmt (MN1 bis MN3, Abb. 15 und 16). Die mesiale Messung (MN1) erfolgte in einem Abstand von 2 mm zum letzten Molaren, während die distale (MN3) am Ende des 20 mm langen Knochenblockes durchgeführt wurde. Dazwischen erfolgte auf halber Länge des Knochenblockes eine weitere Messung (MN2).

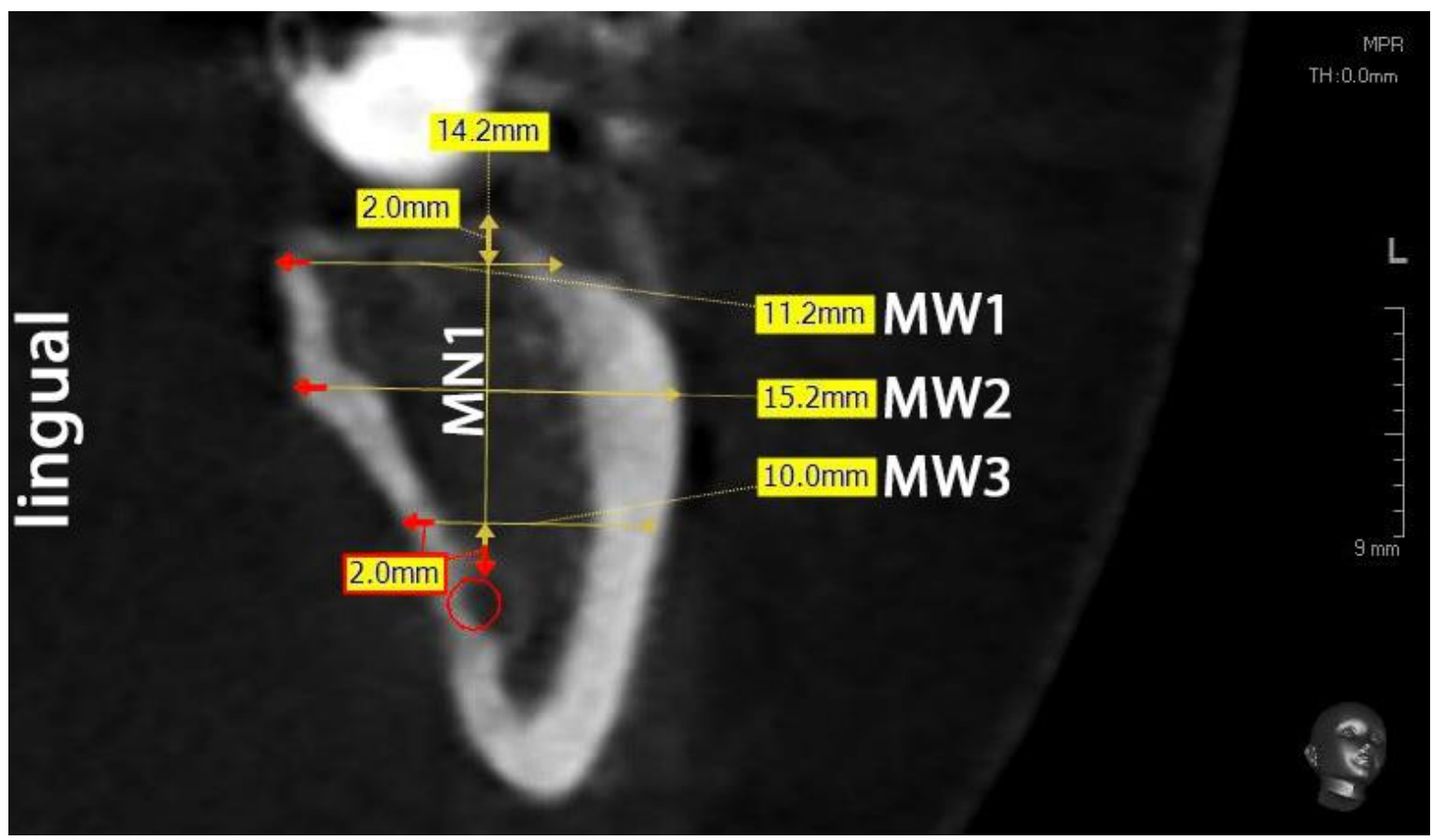
gemessenen Strecken (Klinik für Mund-, Kiefer- und Gesichtschirurgie Universitätsklinikum Göttingen)

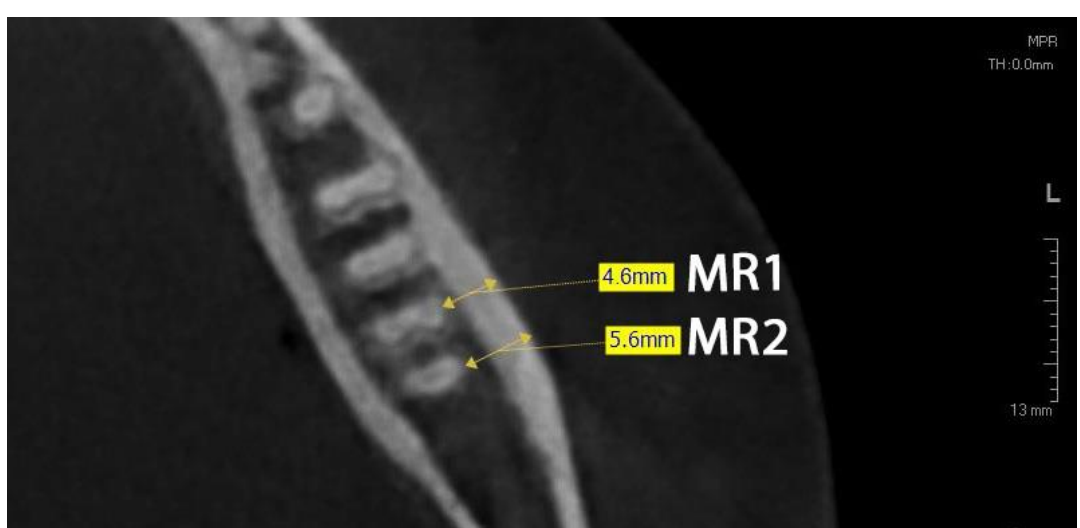


Entlang der drei vertikalen Messungen erfolgte im rechten Winkel die Bestimmung der Alveolarkammbreite an jeweils drei Punkten (MW1 bis MW9, Abb. 16 und 18). Die kraniale Messung (MW1) wurde $2 \mathrm{~mm}$ entfernt von der okklusalen Knochenoberfläche durchgeführt, während die kaudale $2 \mathrm{~mm}$ von dem Nerven entfernt erfolgte (MW3). Auf halber Strecke zwischen Nerv und okklusaler Knochenoberfläche erfolgte die mittige Messung (MW2). Eine Messung dieser Strecken ist in der koronaren Ansicht der DVT-Aufnahmen möglich (Abb. 16). Die Vermessung der bukkalen Knochenwand im Bereich der zweiten Unterkiefermolaren wurde auf halber Wurzellänge in der axialen Ansicht bei beiden Wurzeln (MR1 und MR2, Abb. 17) vorgenommen (Braut et al. 2011; Vera et al. 2012; Wang et al. 2014; Fuentes et al. 2015).

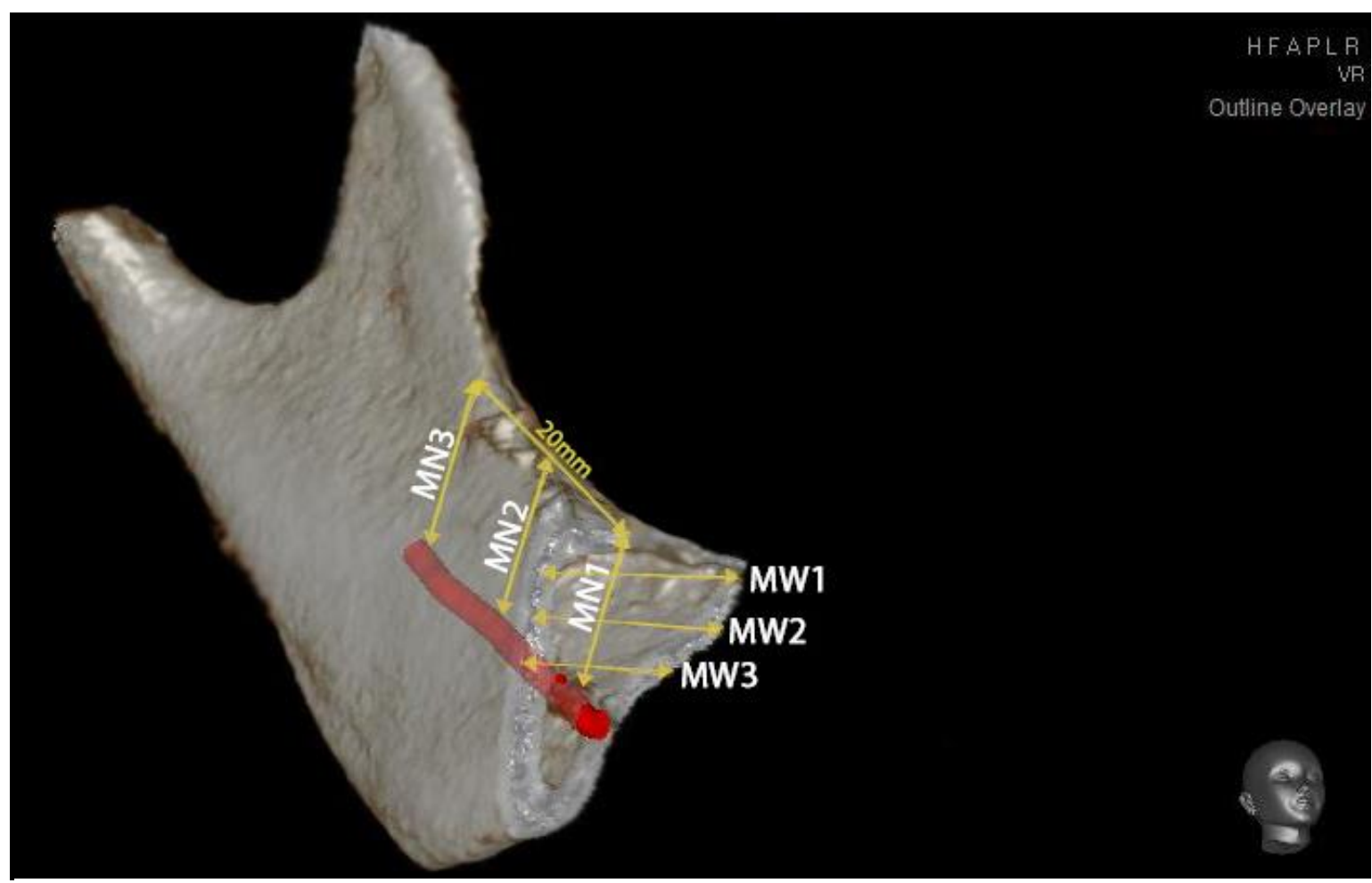
vus alveolaris inferior und gemessenen Dimensionen des Knochenblockes (Klinik für Mund-, Kieferund Gesichtschirurgie Universitätsklinikum Göttingen) 
Als letzte potentielle Knochenentnahmeregion wurde die Crista zygomatico-alveolaris untersucht. Nach Auffinden der Crista in der lateralen knöchernen Begrenzung des Sinus maxillaris, wurde in der Axialansicht der symmetrisch auslaufende Umschlagspunkt markiert (Abb. 19). Ausgehend von diesem, wurde eine dorsale und ventrale Strecke von je $5 \mathrm{~mm}$ eingezeichnet, sodass eine gesamte Transplantatbreite von $10 \mathrm{~mm}$ entstand. Die kaudale Begrenzung des Transplantates stellte entweder der Kieferhöhlenboden oder die Wurzelspitze eines dort befindlichen Molaren dar. Ausgehend von dieser Ebene wurde eine kranio-kaudale Länge von $10 \mathrm{~mm}$ markiert (Abb. 20).
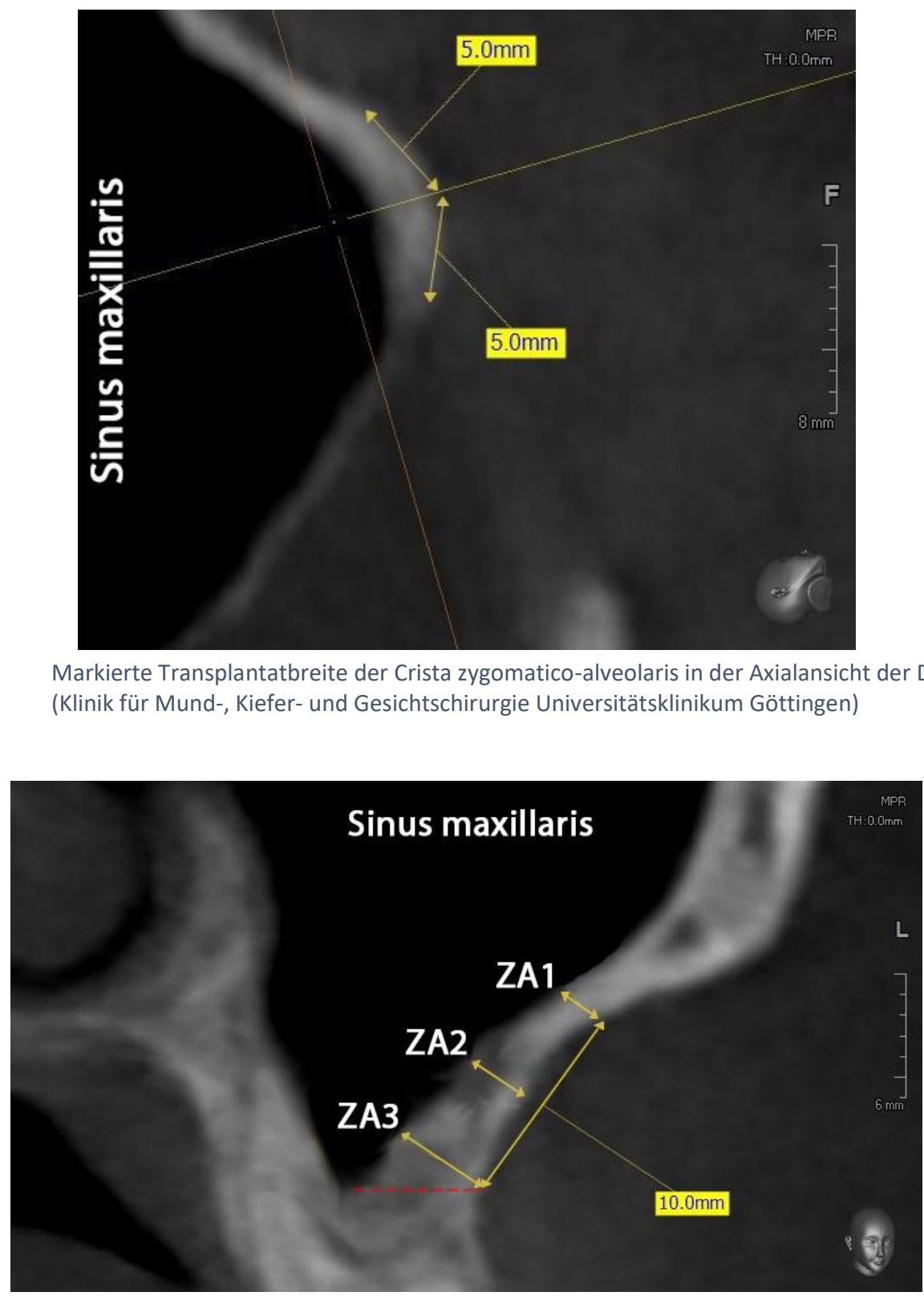
Entlang der markierten Fläche mit einer Länge und Breite von 10 mm wurde an neun Punkten (ZA1 bis ZA9, Abb. 21) die Knochendicke in der koronaren Ansicht gemessen. Hierzu wurde die koronare Schnittebene parallel zum Umschlagspunkt der Crista bewegt (Abb. 20). Die ersten drei Messungen erfolgten entlang der ventralen Begrenzung des Transplantates am kranialen (ZA1) und kaudalen (ZA3) Ende, sowie mittig (ZA2). Dieses Vorgehen wurde an der distalen Begrenzung wiederholt (ZA7 bis ZA9). Entlang des Umschlagpunktes erfolgten ebenfalls drei Messungen (ZA4 bis ZA6).

\section{kranial}

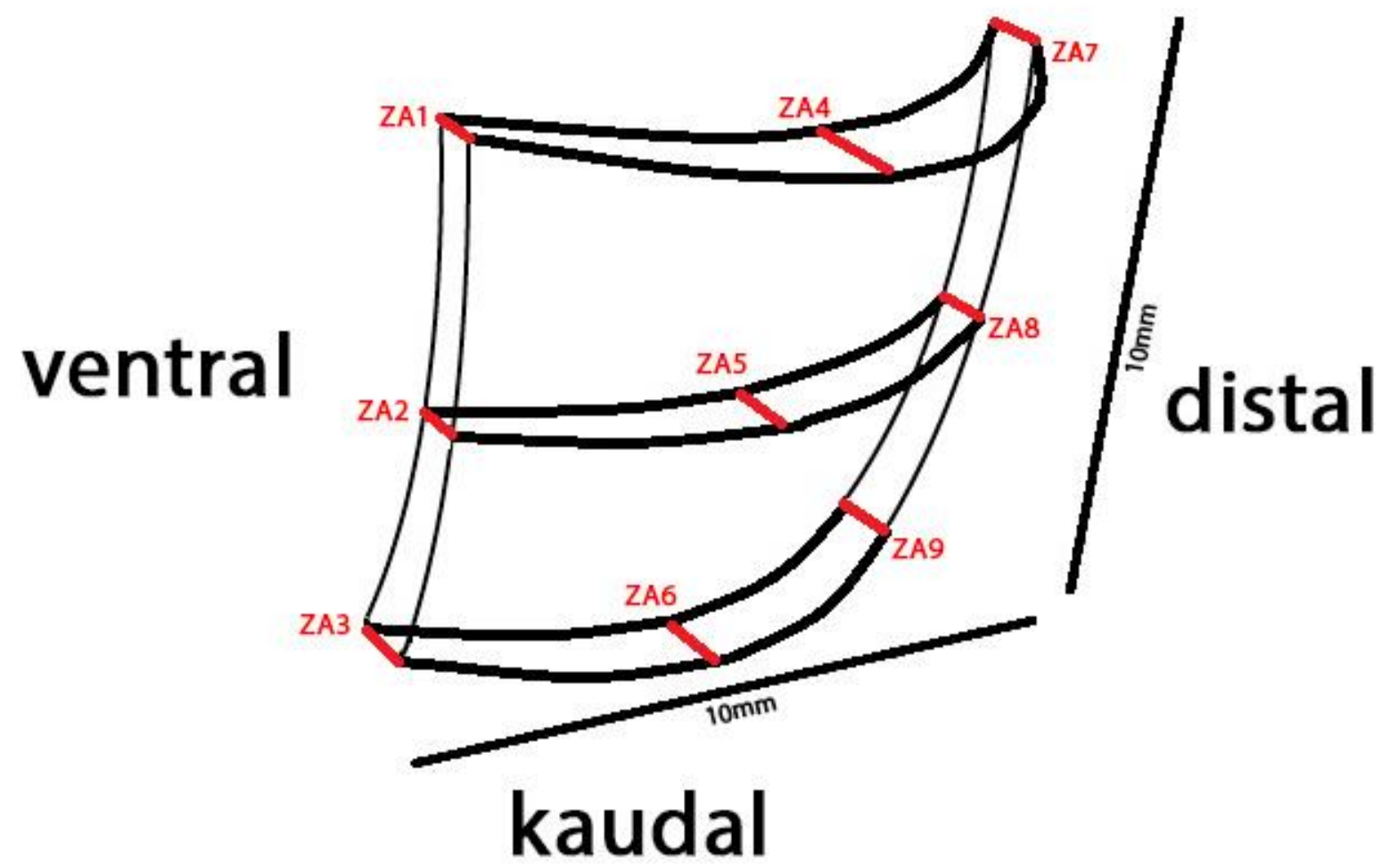


Nach Messung der erwähnten Strecken, wurden die Volumina für alle drei Knochenspenderregionen errechnet. Im Bereich des Kinns wurden hierzu die Mittelwerte für die vertikale Knochenhöhe (CV), horizontale Knochenbreite (CH) und die sagittale Knochentiefe (CS) errechnet und miteinander multipliziert.

$$
\text { Vchin }=\left(\frac{C V 1+C V 2+C V 3}{3}\right) \times\left(\frac{C H 1+C H 2+C H 3}{3}\right) \times\left(\frac{C S 1+C S 2+(\ldots)+C S 9}{9}\right)
$$

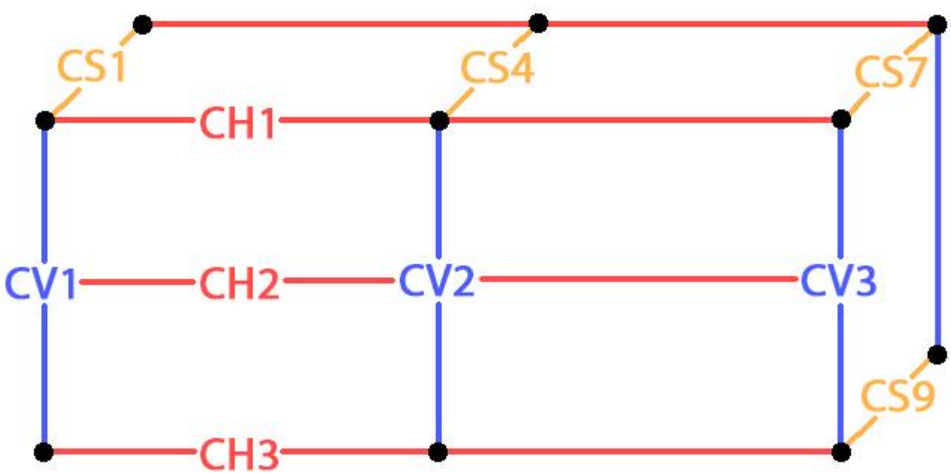

Abbildung 22 Schematische Darstellung der Messungen im Kinn

Für die Volumenbestimmung des retromolaren Knochenblockes wurden die Mittelwerte für die Entfernung des N. alveolaris inferior zu der Knochenoberfläche (MN) und die Breite des Knochenblockes (MW) errechnet und mit der festgelegten Länge von 20 mm multipliziert.

$$
\text { Vretro }=\left(\frac{M N 1+M N 2+M N 3}{3}\right) \times\left(\frac{M W 1+M W 2+(\ldots)+M W 9}{9}\right) \times 20
$$

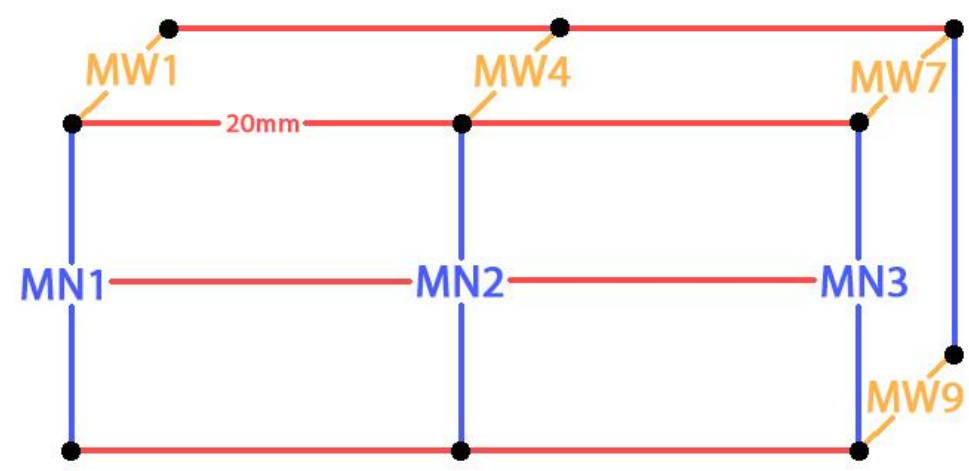


Die Volumenbestimmung im Bereich der Crista zygomatico-alveolaris folgt dem gleichen Prinzip. Höhe und Breite des Knochenblockes sind mit jeweils $10 \mathrm{~mm}$ festgelegt und werden mit der durchschnittlichen Dicke des Transplantates (ZA) multipliziert (siehe Abb. 21).

$$
\text { Vcrista }=\left(\frac{Z A 1+Z A 2+(\ldots)+Z A 9}{9}\right) \times 10 \times 10
$$

Neben den erwähnten Strecken und Volumina wurde der Verlauf des Nervus alveolaris inferior untersucht und in zwei Gruppen eingeteilt. Hierzu wurde in der axialen Ansicht der DVT-Aufnahme ein Abstand von $5 \mathrm{~mm}$ distal des letzten Molaren markiert (Abb. 24). Anhand dieses Abstandes konnte eine koronare Ansicht eingestellt werden, die zur Beurteilung des Nervverlaufes diente (Abb. 25). Die Einteilung in zwei Gruppen erfolgte je nachdem ob der Nerv in der lingualen oder bukkalen Hälfte der Mandibula verlief.

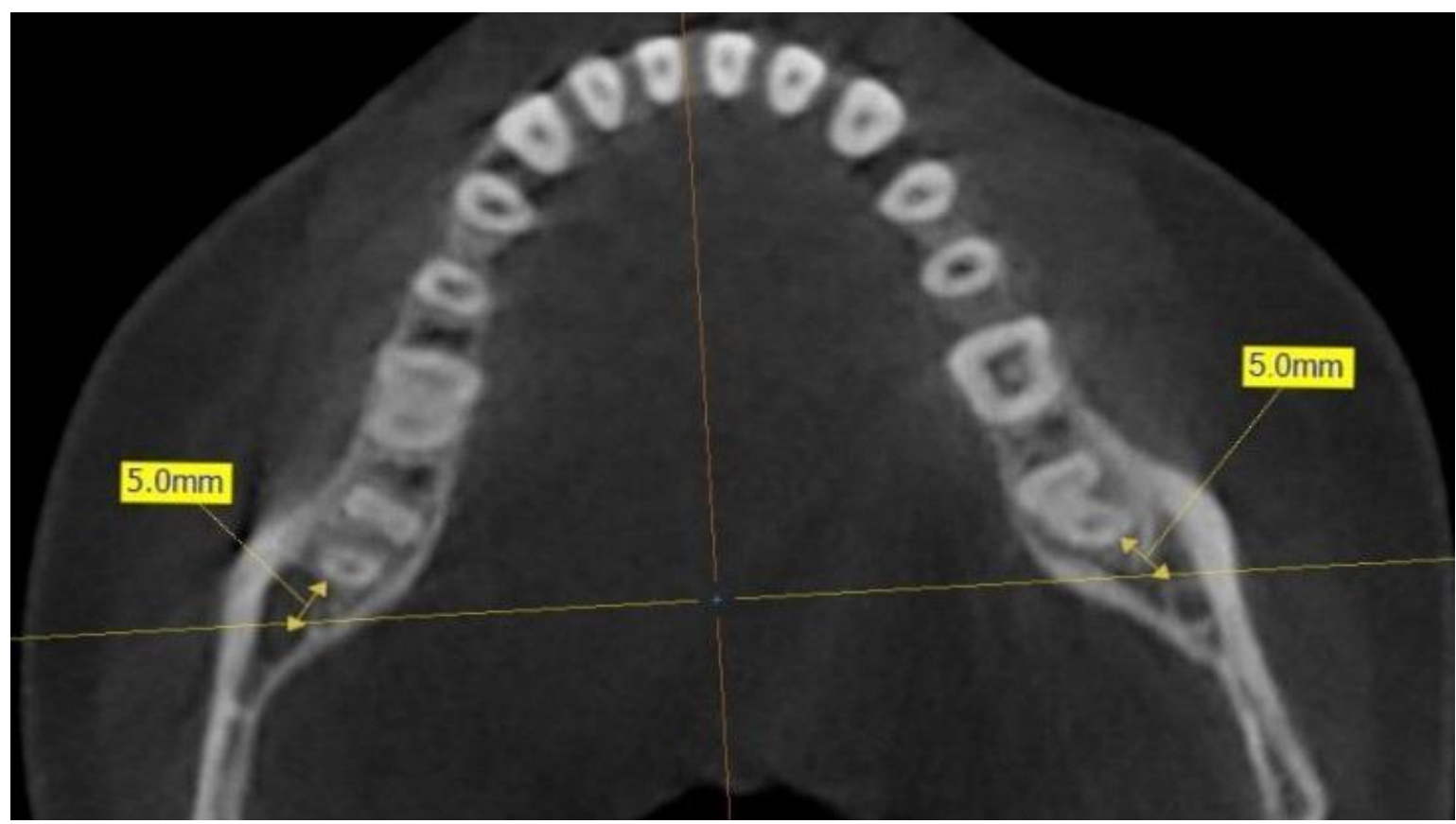
Die Einstellung der koronaren Ansicht erfolgte mit Hilfe dieser Abstandsmarkierungen (Klinik für Mund-, Kiefer- und Gesichtschirurgie Universitätsklinikum Göttingen) 


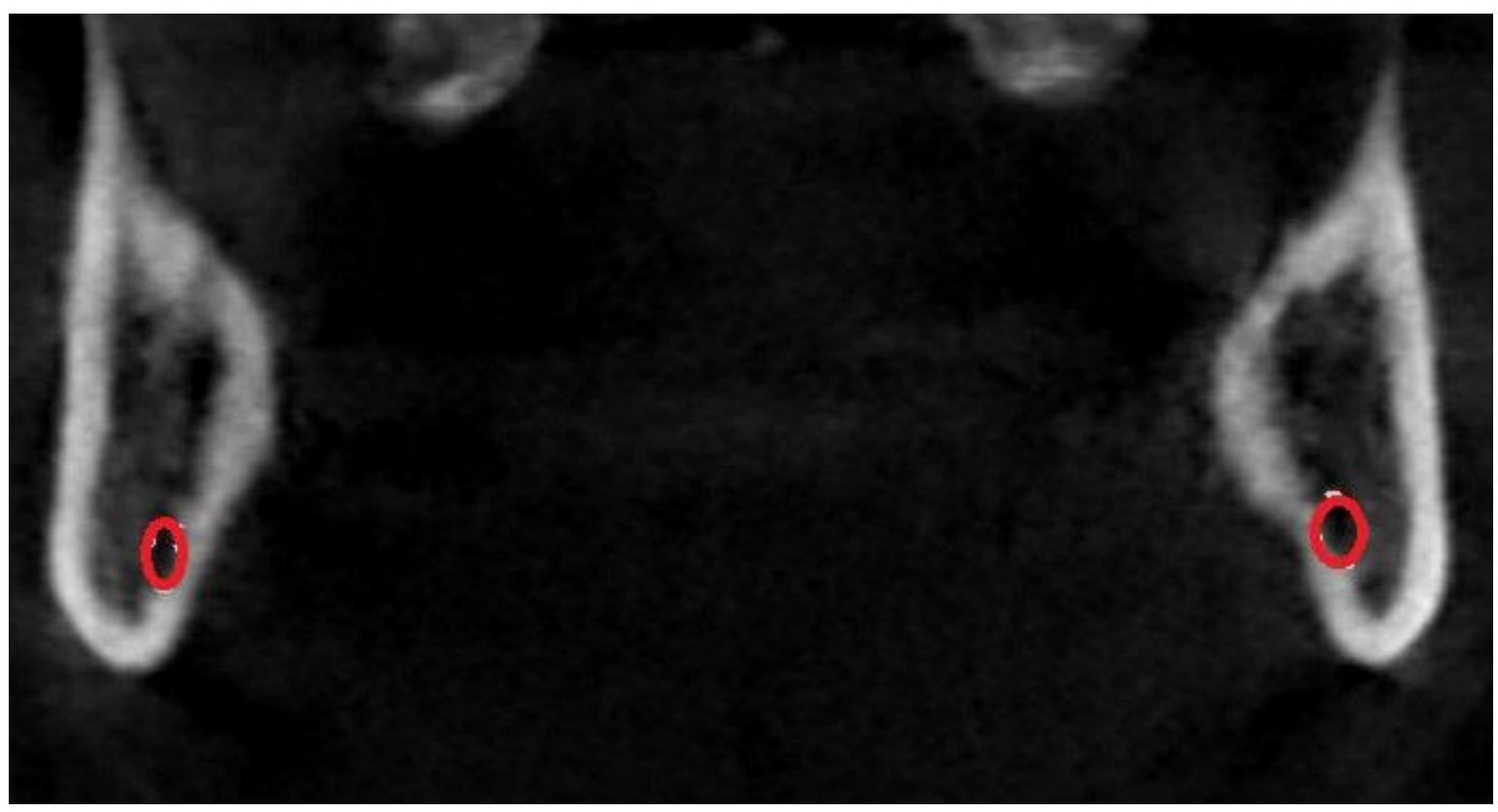

Abbildung 26

Koronare Ansicht der Mandibula im DVT. Der lingual verlaufende Nervus alveolaris inferior ist rot markiert (Klinik für Mund-, Kiefer- und Gesichtschirurgie Universitätsklinikum Göttingen)

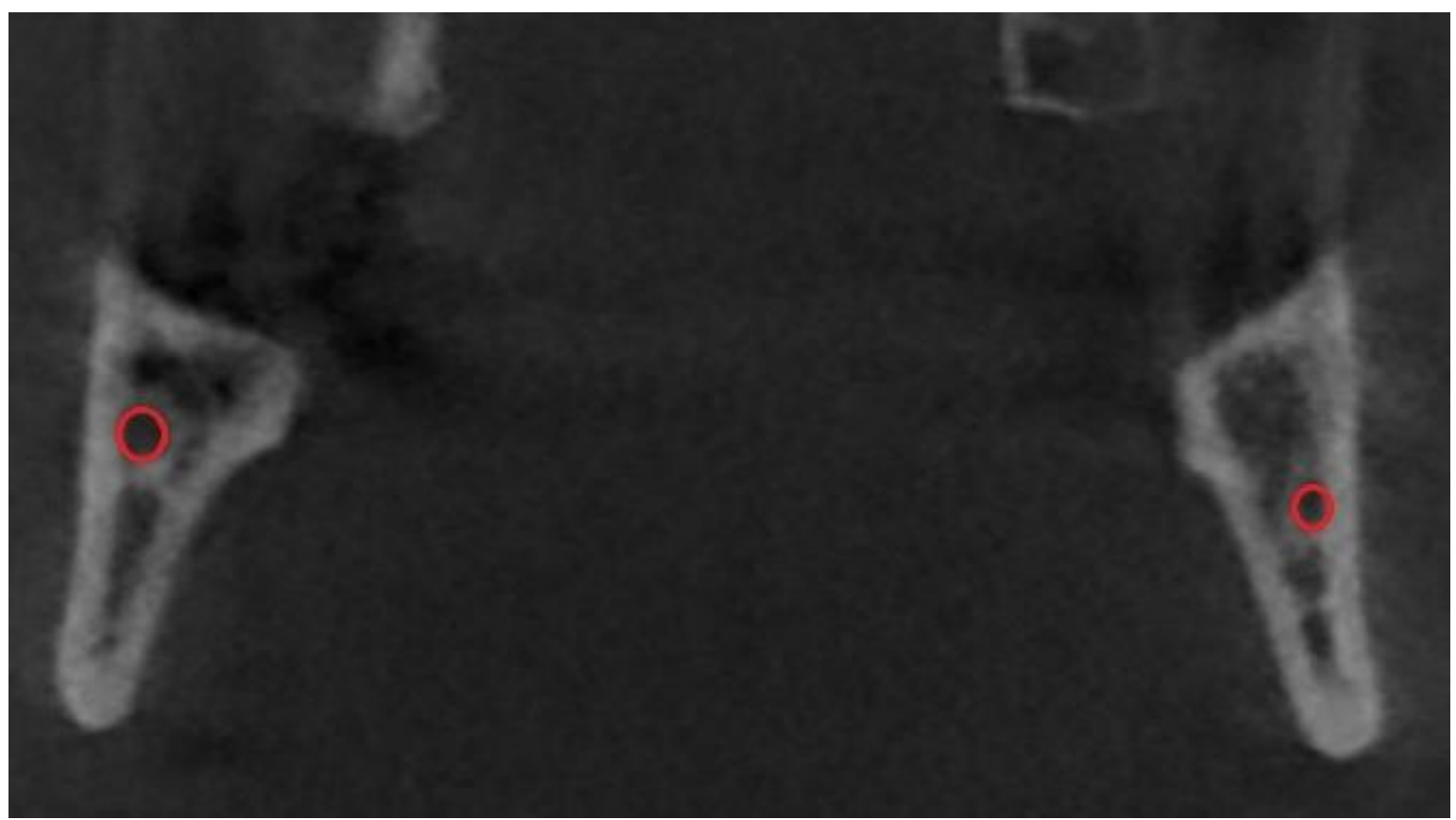

Abbildung 25

Koronare Ansicht der Mandibula im DVT. Der bukkal verlaufende Nervus alveolaris inferior ist rot markiert (Klinik für Mund-, Kiefer- und Gesichtschirurgie Universitätsklinikum Göttingen) 


\subsection{Statistische Methoden}

Für die statistische Auswertung der gewonnenen Daten und Erstellung der Grafiken wurde mit der Statistiksoftware STATISTICA ${ }^{\circledR}$ (Firma StatSoft Europe GmbH, Hamburg, Deutschland) und SPSS ${ }^{\circledR}$ (Firma IBM Corporation, New York, USA) gearbeitet.

Box-Whisker-Plots, Scatterplots und Säulendiagramme wurden für die grafische Darstellung verwendet. Die erhobenen Daten wurden mit Hilfe von Shapiro-Wilk-Tests auf Normalverteilung geprüft. Die Ermittlung der statistischen Signifikanz ( $p-W e r t)$ bzw. Korrelationen erfolgte durch „student's t-tests“, „Chi-Quadrat-Tests“, lineare Regressionen, logistische Regressionen mit backward removal Algorithmus und die „Pearson-Korrelation“. Für die Untersuchung der Interrater-Reliabilität wurden Bland-Altman-Plots erstellt.

Diese Arbeitsschritte erfolgten im Rahmen des betreuten Arbeitens am Institut für medizinische Statistik der Universitätsmedizin Göttingen. 


\section{Ergebnisse}

\subsection{Deskriptive Statistik}

Für diese Arbeit wurden 108 Röntgenaufnahmen von 36 Patienten untersucht. Die drei Aufnahmen pro Patient setzen sich aus einer OPG-, FRS- und DVT-Aufnahme zusammen. 20 der Patienten waren weiblich, 16 männlich. Das Patientenalter lag zu dem Zeitpunkt der Röntgenaufnahme zwischen 18 und 51 Jahren mit einem Mittelwert von 25,8 Jahren. Einen genaueren Einblick erlaubt das Histogramm (Abb. 27).

Anzahl der untersuchten Aufnahmen [n]

\begin{tabular}{|c|c|}
\hline Aufnahmemethode & $\mathbf{n}$ \\
\hline OPG & 36 \\
\hline FRS & 36 \\
\hline DVT & 36 \\
\hline gesamt & 108 \\
\hline
\end{tabular}

Tabelle 2

Anzahl weiblicher und männlicher Patienten [n]

\begin{tabular}{|c|c|}
\hline Geschlecht & $\mathbf{n}$ \\
\hline Weiblich & 20 \\
\hline Männlich & 16 \\
\hline gesamt & 36 \\
\hline
\end{tabular}

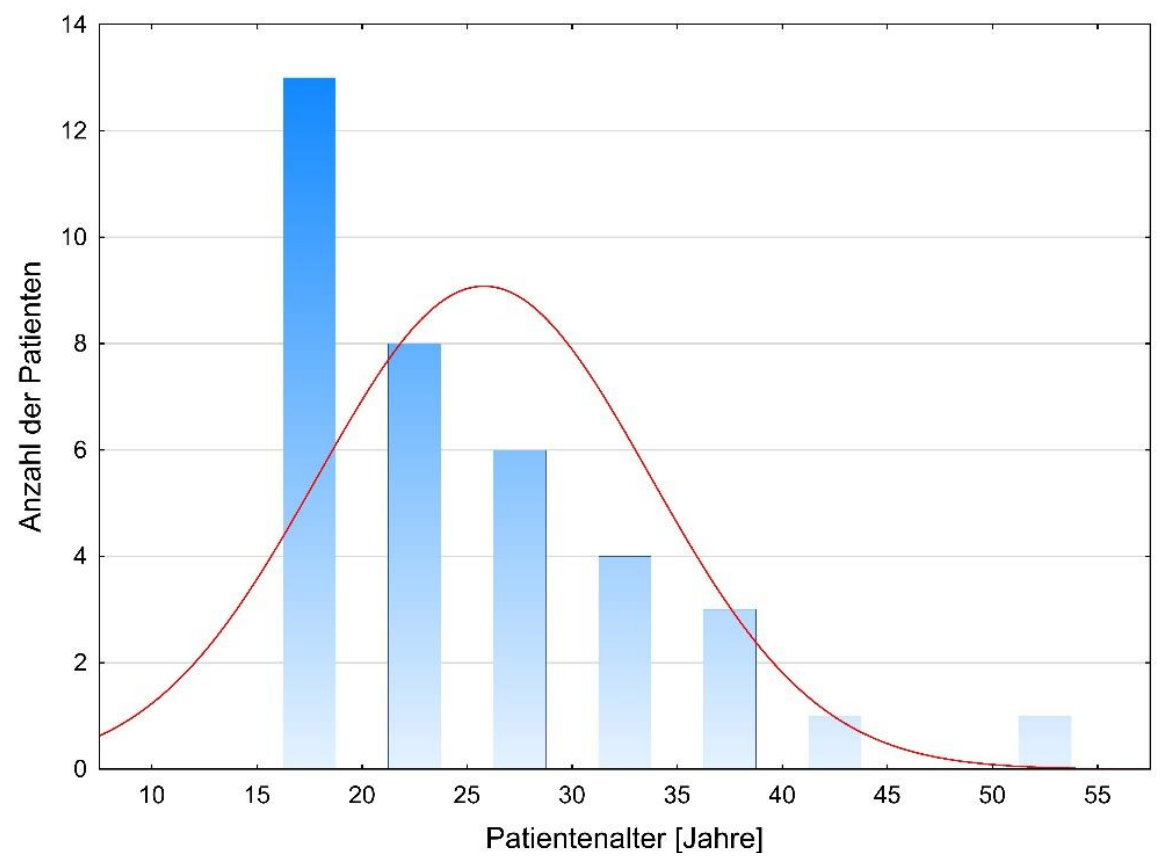




\subsubsection{FRS-Messungen}

In jedem FRS wurden acht Winkel gemessen, der Index aus oberer und unterer Gesichtshöhe bestimmt und die strukturelle Methode nach Björk modifiziert nach Hasund angewandt (im Rahmen dieser Arbeit als „Hasund“ bezeichnet). Folgende Tabelle dient der übersichtlichen Darstellung der aufgerundeten Messergebnisse. Zur graphischen Darstellung der kephalometrischen FRS-Winkel, sowie von Index und „Hasund-Score“ wurden drei Box-Whisker-Plots erstellt.

Tabelle 3 Deskriptive Statistik für sämtliche Messungen im FRS. Die Werte SNA, SNB, ANB, ML-NSL, NL-NSL, $\mathrm{ML}-\mathrm{NL}, \mathrm{NSBa}$ und Gn-Go-Ar sind in Grad [ $\left.{ }^{\circ}\right]$ angegeben. Die Angabe des Index erfolgt in Prozent [\%]. Der Hasund Wert ist in Punkten angegeben.

\begin{tabular}{|c|c|c|c|c|c|}
\hline Parameter & $\mathbf{n}$ & Mittelwert $\left[^{\circ}\right.$ ] & Minimum [ ${ }^{\circ}$ ] & Maximum [ & SD $\left.^{\circ}{ }^{\circ}\right]^{\circ}$ \\
\hline SNA & 36 & 80,16 & 68,70 & 90,90 & 5,26 \\
\hline SNB & 36 & 78,16 & 62,70 & 94,25 & 8,28 \\
\hline ANB & 36 & 1,88 & -14 & 12,60 & 6,23 \\
\hline ML-NSL & 36 & 33,26 & 10,05 & 51,25 & 10,43 \\
\hline NL-NSL & 36 & 9,11 & 1,20 & 21,55 & 4,55 \\
\hline ML-NL & 36 & 24,15 & 2,15 & 42,65 & 9,22 \\
\hline NSBa & 36 & 131,89 & 117,85 & 168 & 9,30 \\
\hline Gn-Go-Ar & 36 & 125,33 & 103,05 & 138,95 & 8,57 \\
\hline Index [\%] & 36 & 77,36 & 64 & 101 & 7,97 \\
\hline Hasund [Punkte] & 36 & 2,47 & -12 & 17 & 6,96 \\
\hline
\end{tabular}

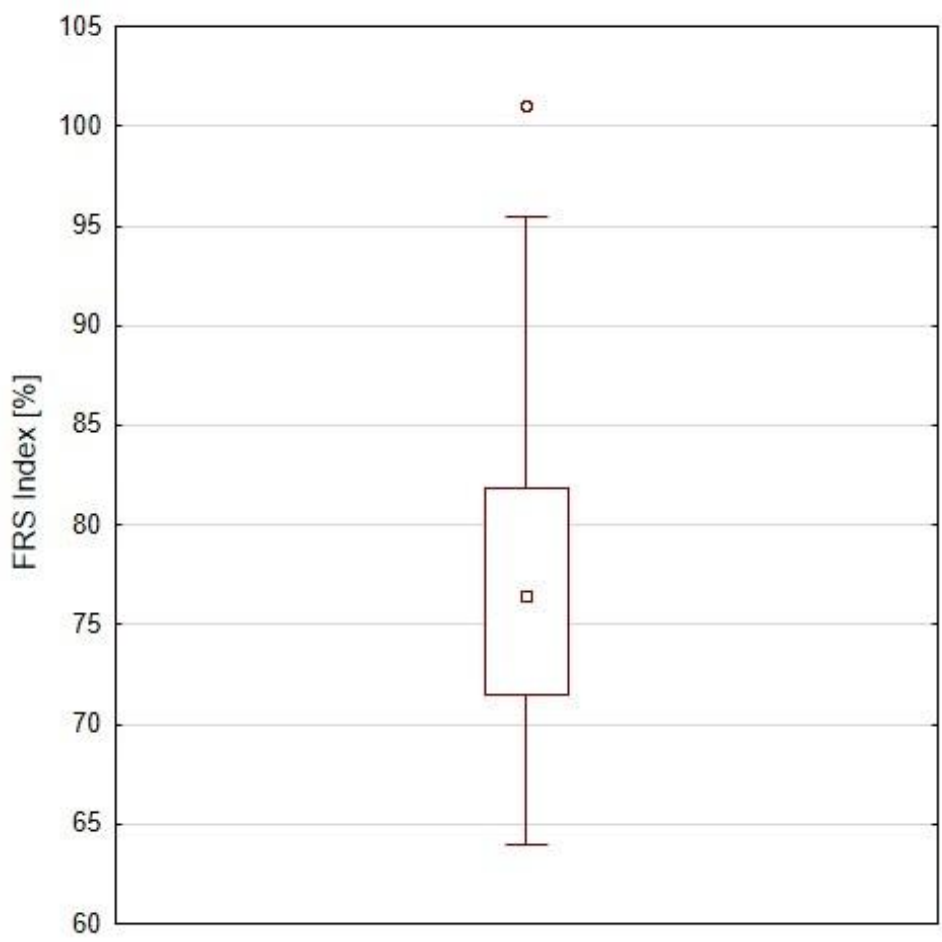




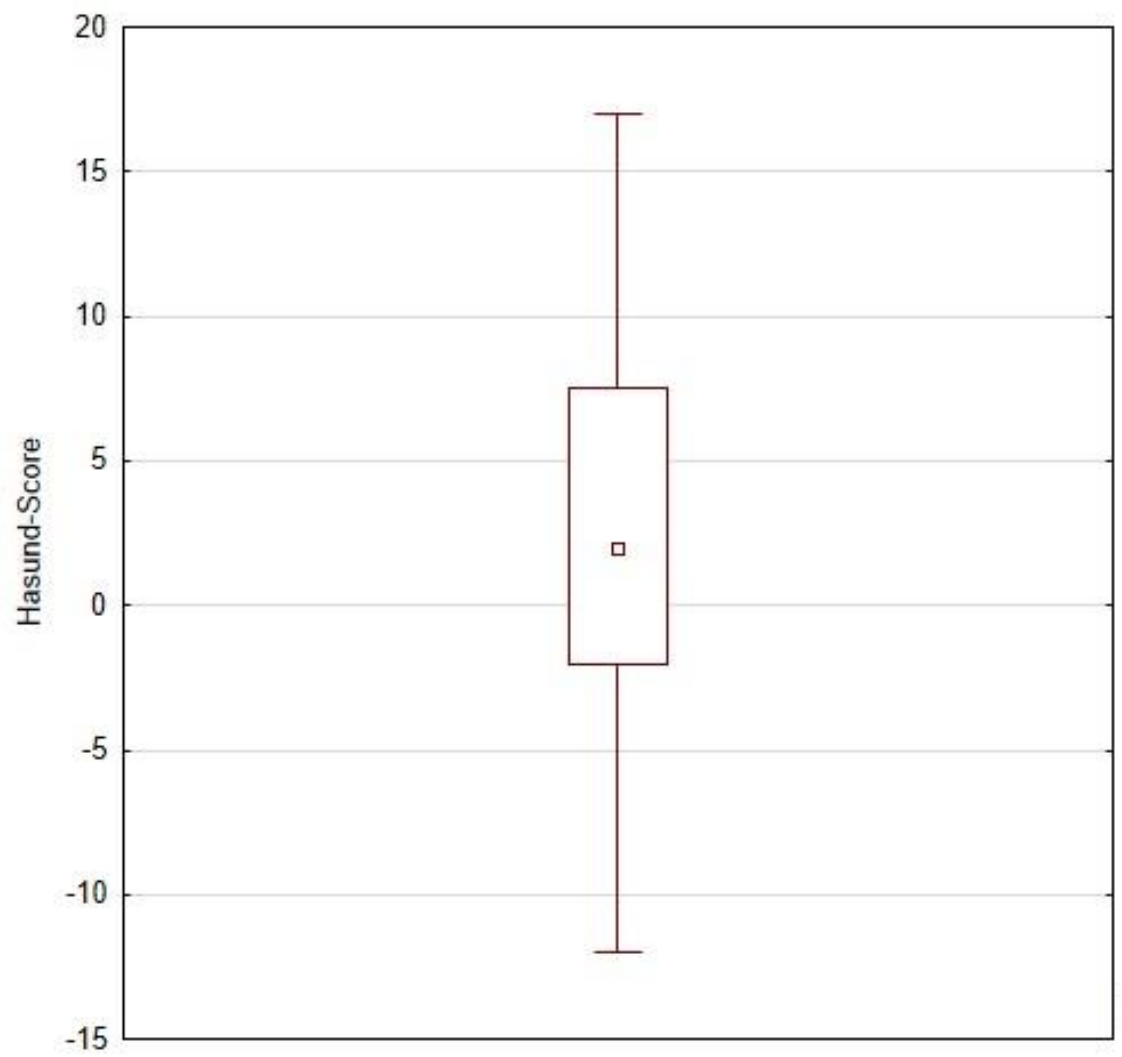

Abbildung 29

Box-Whisker-Plot des Hasund-Scores

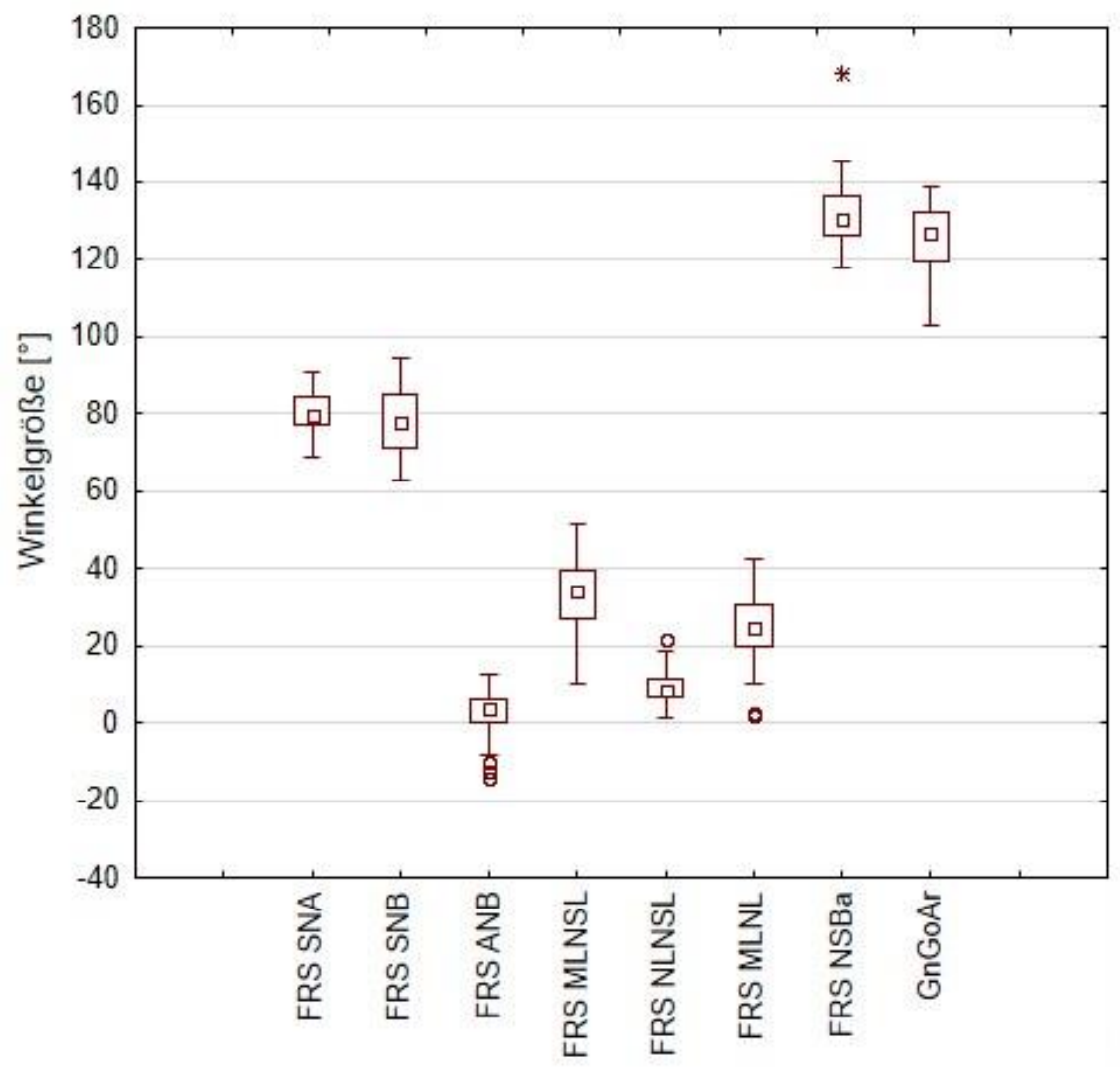




\subsubsection{OPG-Messungen}

In jedem OPG wurde der Mittelwert beider Kieferwinkel bestimmt. Außerdem wurde die Entfernung der Wurzelspitzen der mittleren Unterkiefer Incisivi (WS1) und der zweiten Molaren (WS7) zur Unterkieferbasis gemessen (Abb. 11). Ein Quotient beider Strecken wurde errechnet. Anhand der Tabelle 4 und den Box-Whisker-Plots lässt sich erkennen, dass im OPG zwischen den Wurzelspitzen der mittleren Unterkiefer Incisivi und dem Unterrand der Mandibula größere Strecken (WS1) gemessen wurden, als zwischen den Wurzelspitzen der zweiten Molaren und dem Unterrand der Mandibula (WS7). Im OPG gemessen war das durchschnittliche vertikale Knochenangebot im Bereich des Kinns also größer als im Bereich der zweiten Molaren. Allerdings wurden für die vertikalen Messungen im Kinn größere Standardabweichungen festgestellt als im Molarenbereich.

Tabelle 4 Deskriptive Statistik für sämtliche Messungen im OPG. WS1, WS7 und Quotient sind in [mm] angegeben. Ang. Mand. ist in Grad $\left[^{\circ}\right]$ angegeben.

\begin{tabular}{|c|c|c|c|c|c|}
\hline Parameter & $\mathbf{n}$ & Mittelwert [mm] & Minimum [mm] & Maximum [mm] & SD [mm] \\
\hline WS1 & 36 & 25,39 & 14,37 & 34,02 & 4,80 \\
\hline WS7 & 36 & 13,19 & 7,50 & 20,53 & 3,28 \\
\hline Quotient & 36 & 2,02 & 0,90 & 2,95 & 0,52 \\
\hline Ang. Mand. [ ${ }^{\circ}$ ] & 36 & 127,50 & 111,08 & 140,40 & 7,20 \\
\hline
\end{tabular}

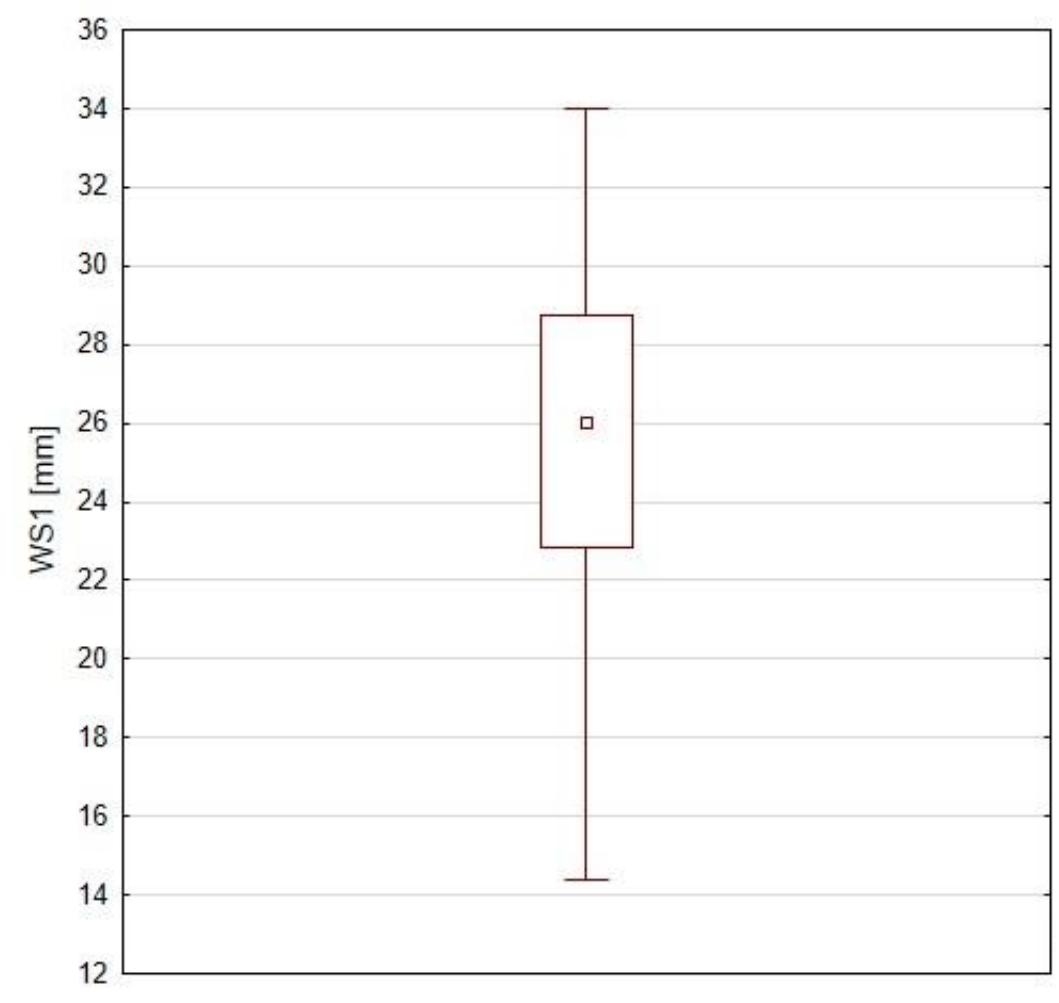




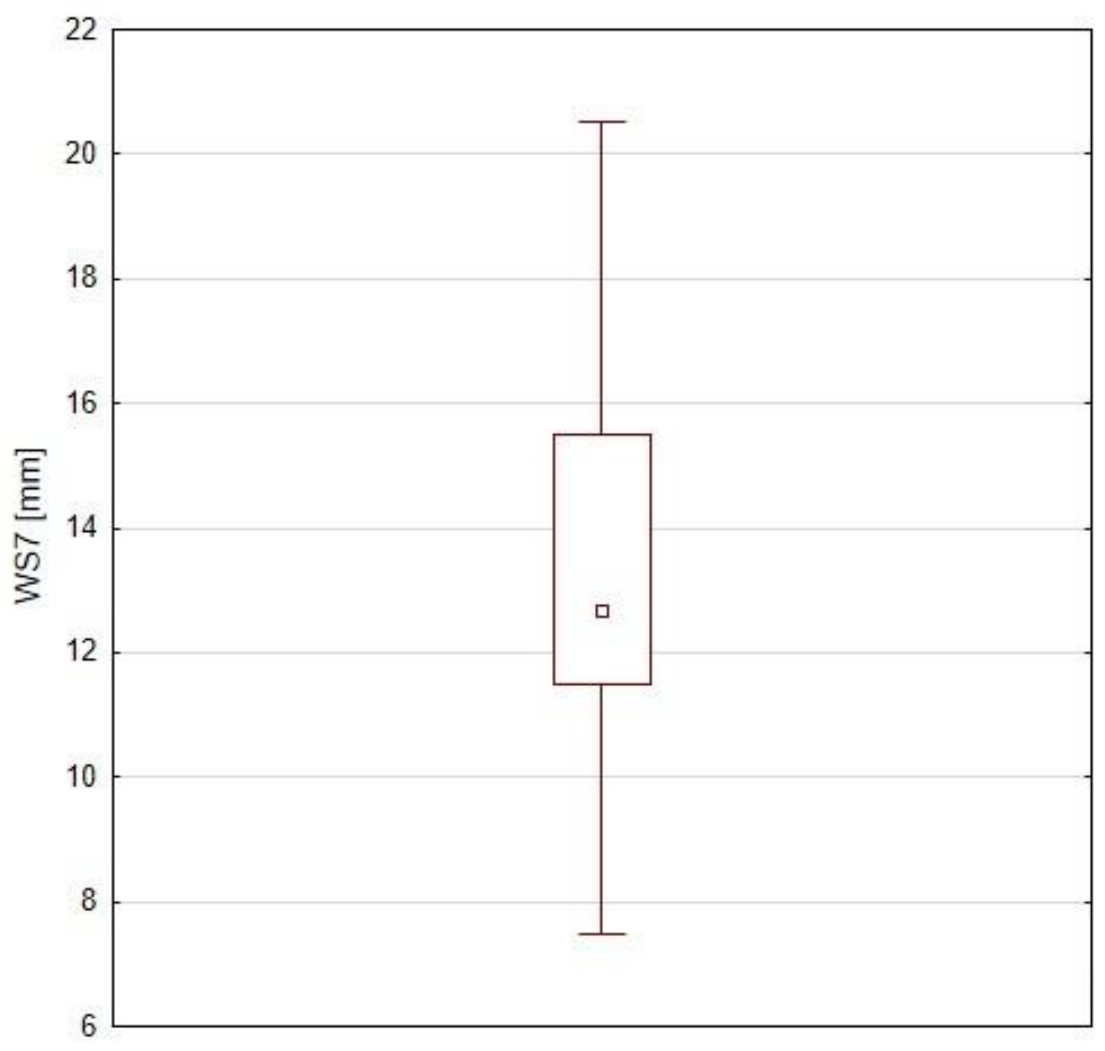

Abbildung 32 Box-Whisker-Plot des im OPG gemessenen WS7 in [mm]

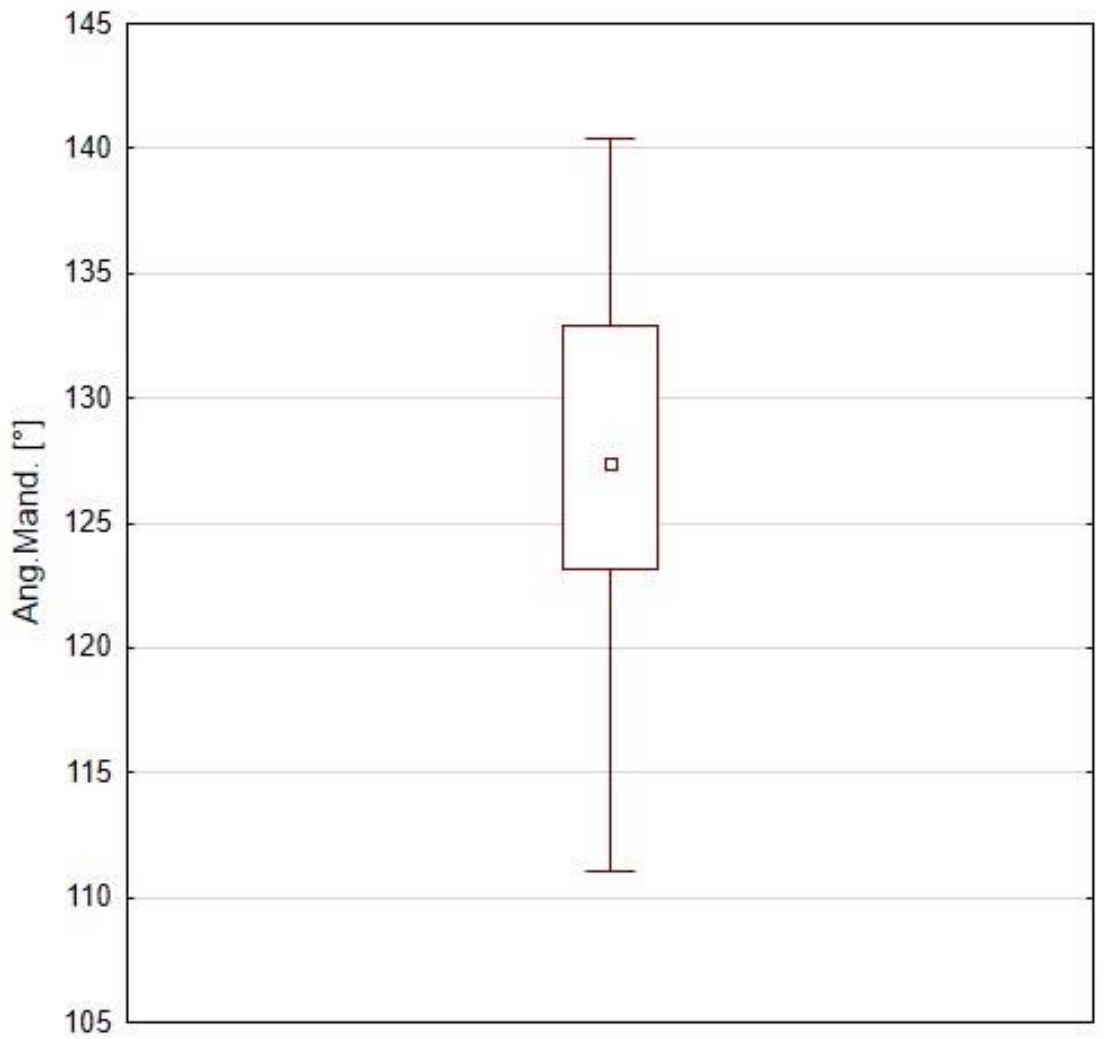




\subsubsection{Messungen am DVT}

Da in den DVT-Aufnahmen drei verschiedene potentielle Knochenentnahmeregionen untersucht worden sind, werden diese in den folgenden Tabellen und Plots getrennt dargestellt.

\subsubsection{DVT-Messungen des Kinns}

Die Vermessungen des Kinns erfolgten durch Bestimmung der horizontalen Ausdehnung in drei unterschiedlichen Höhen ( $\mathrm{CH} 1$ bis $\mathrm{CH} 3$ ). Die vertikale Dimension des Kinns wurde ebenfalls an drei Stellen gemessen (CV1 bis CV3). Hieraus ergeben sich insgesamt neun Schnittpunkte (CS1 bis CS9), an denen die sagittale Dicke des Kinns abschließend gemessen wurde (Abb. 14). Das Knochenvolumen des Kinns (Vchin) wurde aus diesen Messungen errechnet. Eine schematische Darstellung kann der Abbildung 22 entnommen werden. Die deskriptive Statistik ist in folgender Tabelle zusammengefasst. 
Tabelle 5 Deskriptive Statistik der DVT-Messungen im Kinn. Die Parameter CH, CV und CS sind in [mm] angegeben. Der Parameter Vchin ist in $\left[\mathrm{cm}^{3}\right]$ angegeben.

\begin{tabular}{|c|c|c|c|c|c|}
\hline Parameter & $\mathbf{n}$ & Mittelwert [mm] & Minimum [mm] & Maximum [mm] & SD [mm] \\
\hline $\mathrm{CH} 1$ & 36 & 42,10 & 34,40 & 54,40 & 4,61 \\
\hline $\mathrm{CH} 2$ & 36 & 43,02 & 33,20 & 54,70 & 4,93 \\
\hline $\mathrm{CH} 3$ & 36 & 39,58 & 24,40 & 54,20 & 6,37 \\
\hline CV1 & 36 & 9,64 & 2,40 & 15,50 & 3,24 \\
\hline $\mathrm{CV} 2$ & 36 & 13,70 & 7,30 & 21,20 & 3,51 \\
\hline CV3 & 36 & 9,46 & 0,90 & 16,10 & 3,52 \\
\hline CS1 & 36 & 6,90 & 0,90 & 11,00 & 1,85 \\
\hline CS2 & 36 & 7,31 & 4,50 & 11,00 & 1,58 \\
\hline CS3 & 36 & 4,63 & 2,00 & 7,40 & 1,13 \\
\hline CS4 & 36 & 7,06 & 1,60 & 11,50 & 2,34 \\
\hline CS5 & 36 & 10,12 & 7,40 & 14,00 & 1,69 \\
\hline CS6 & 36 & 5,89 & 3,80 & 8,10 & 1,04 \\
\hline CS7 & 36 & 7,05 & 3,60 & 10,00 & 1,57 \\
\hline CS8 & 36 & 7,92 & 3,70 & 13,20 & 1,80 \\
\hline CS9 & 36 & 5,06 & 3,10 & 8,10 & 1,15 \\
\hline Vchin $\left[\mathrm{cm}^{3}\right]$ & 36 & 3,10 & 1,59 & 6,16 & 1,11 \\
\hline
\end{tabular}

Die horizontale Ausdehnung des knöchernen Kinns ist in der mittleren Position $(\mathrm{CH} 2)$ durchschnittlich am größten. Die größte Standardabweichung unter den horizontalen Messungen liegt für $\mathrm{CH} 3$ vor. Mit einem Abstand von nur $2 \mathrm{~mm}$ zur Unterkieferbasis war diese die kaudalste der drei horizontalen Messungen und lag in unmittelbarer Nähe zu der knöchernen Basis des Kinns, die interindividuell größeren Standardabweichung unterworfen zu sein scheint. Bei dem Vergleich der Vertikalen Messungen fällt auf, dass die mittlere Messposition (CV2) den höchsten Mittelwert aufweist. Bei Einhalten eines Sicherheitsabstandes zu dem Apex (hier $5 \mathrm{~mm}$ ) bietet das Kinn in dem Bereich der Unterkiefer Schneidezähne in der Vertikalen durchschnittlich mehr Knochen, als unterhalb der Eckzähne. Die Mittelwerte für CV1 und CV3 weisen aufgrund der Seitensymmetrie kaum eine Differenz auf. Ähnlich verhält es sich bei den sagittalen Messungen. Die Knochendicken unterhalb der Eckzähne liegen im Seitenvergleich aufgrund der Symmetrie ebenso nahe beieinander. Die durchschnittlich größte Knochendicke wurde bei CS5 gemessen. Die Berechnungen für das Knochenvolumen im Kinn ergaben einen Mittelwert von 3,10 $\mathrm{cm}^{3}$ mit einer Standardabweichung von 1,11 $\mathrm{cm}^{3}$. Die graphische Darstellung der Messergebnisse erfolgt in Box-Whisker-Plots. 


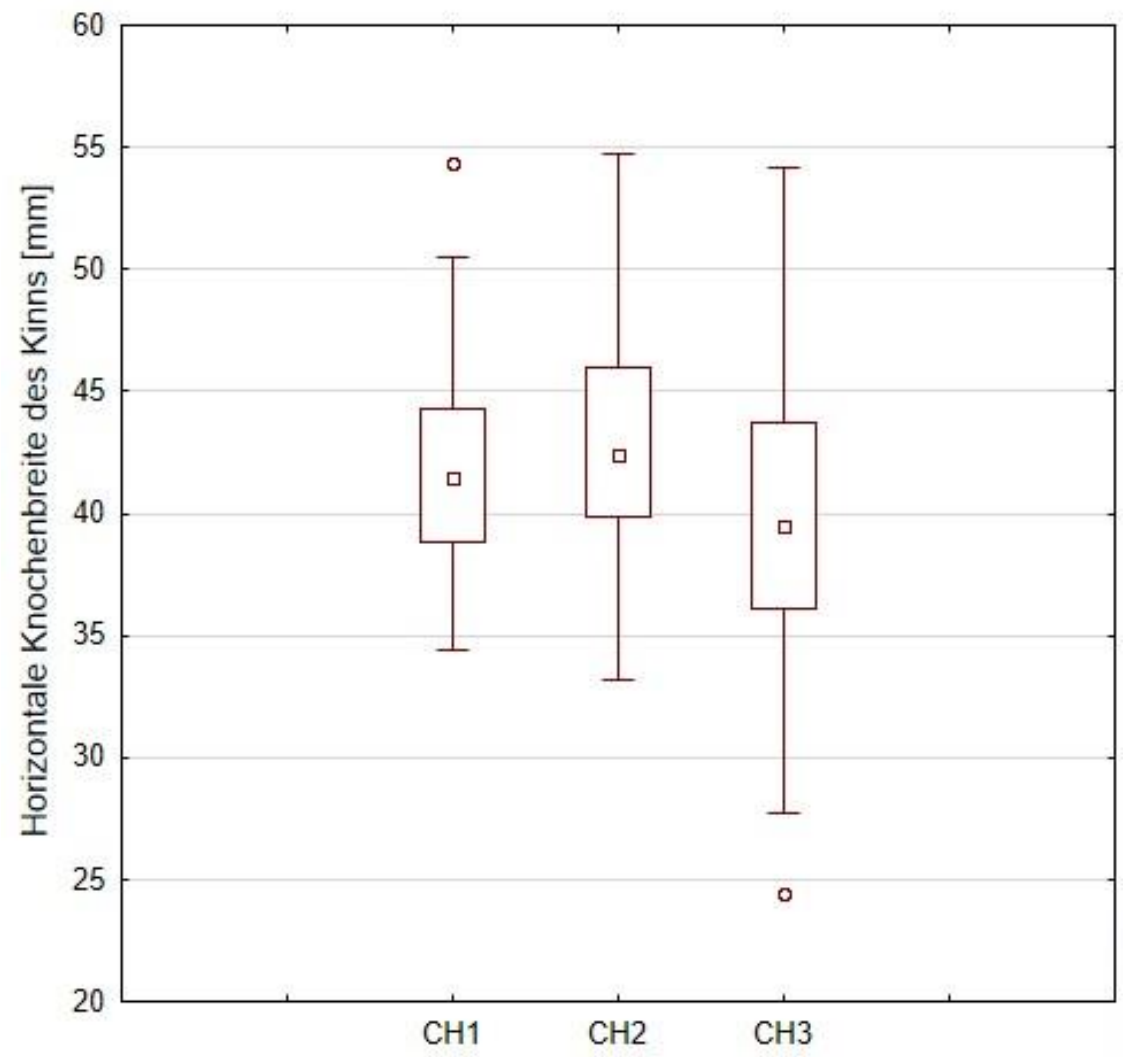

Abbildung 35

Box-Whisker-Plot der transversalen Knochenbreite des Kinns [mm] in drei Lagen ( $\mathrm{CH} 1$ bis $\mathrm{CH} 3$ ) gemessen in DVT-Aufnahmen

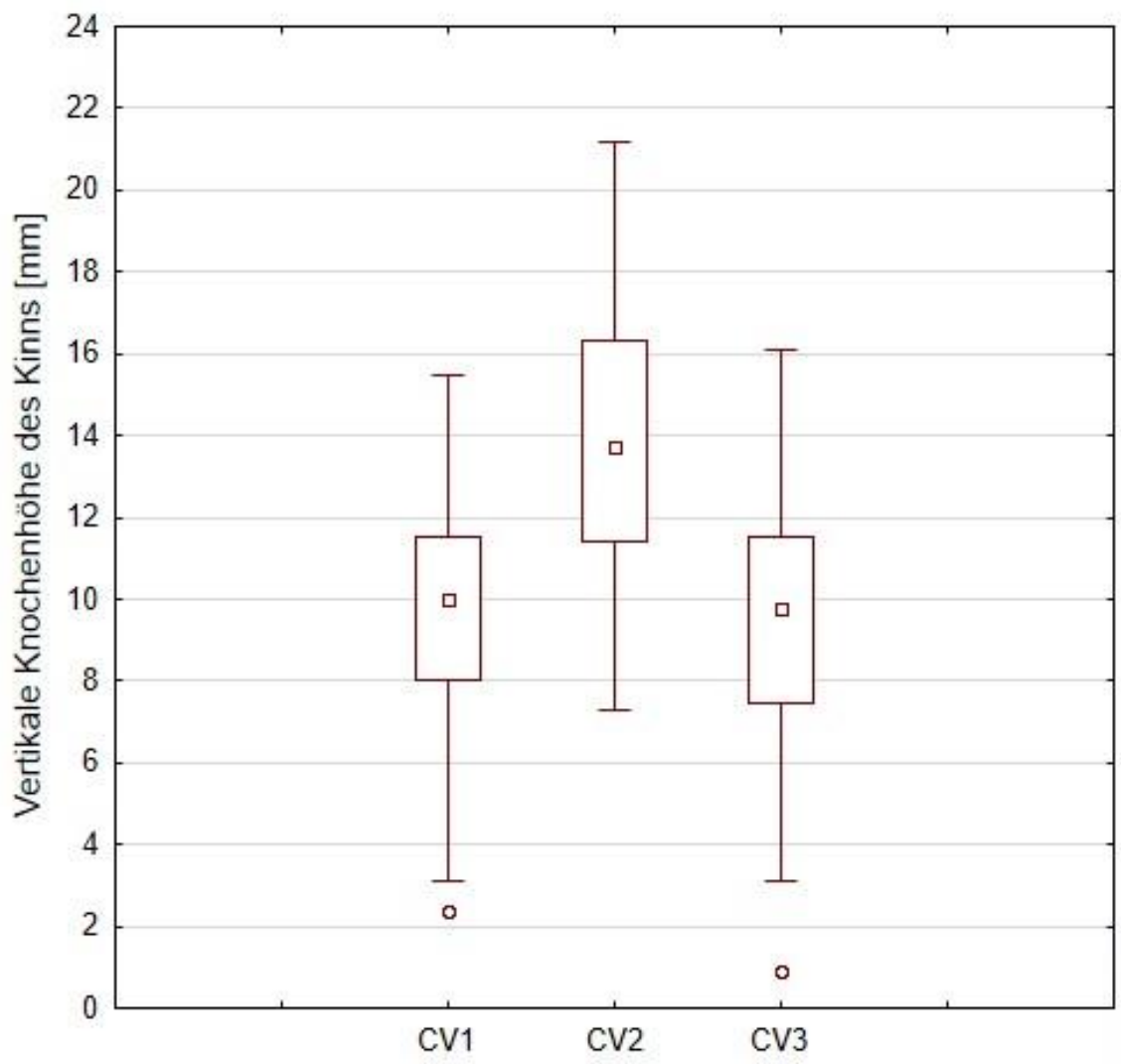
gemessen in DVT-Aufnahmen 


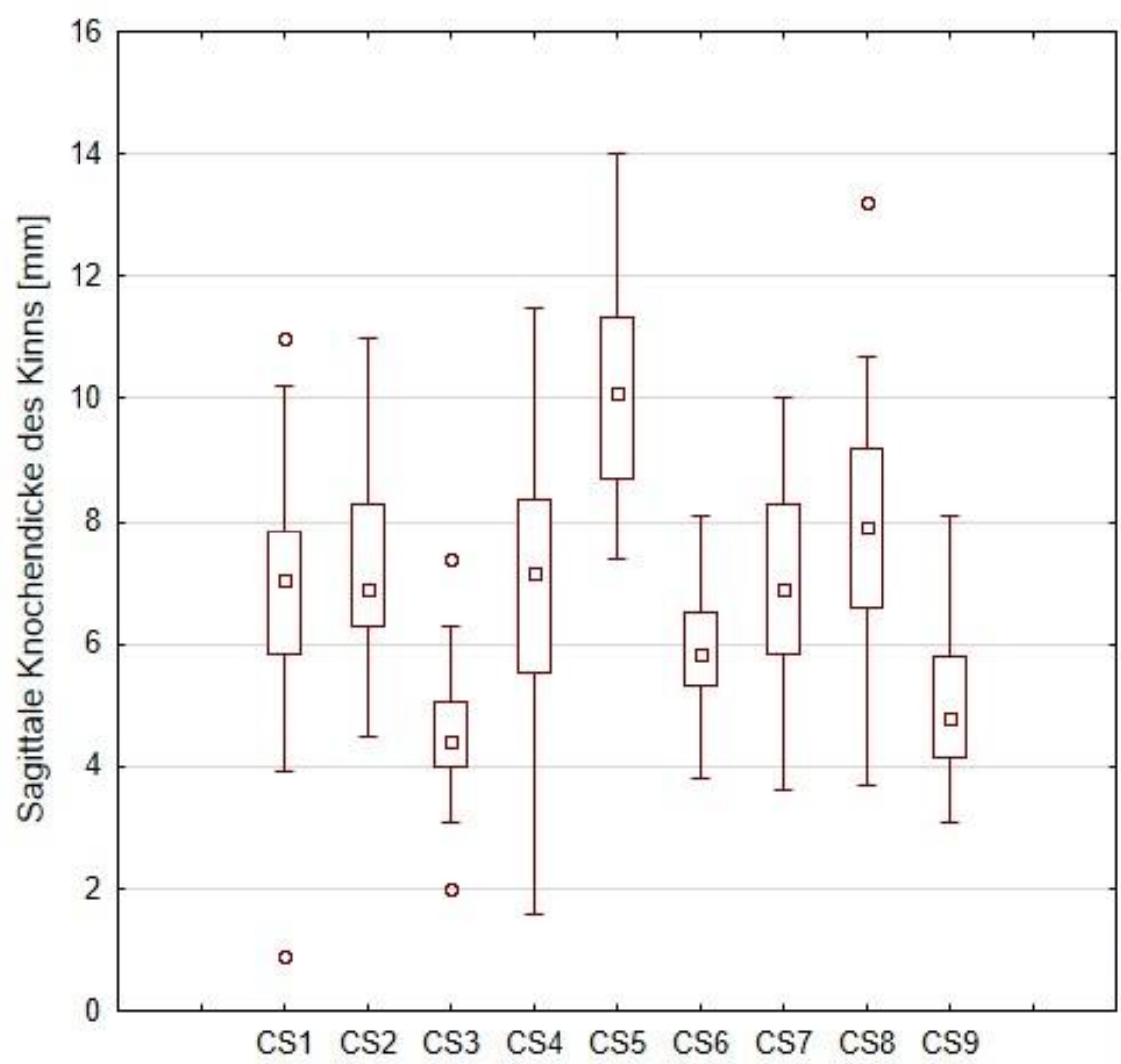

Abbildung 36

Box-Whisker-Plot der Knochendicke des Kinns [mm] an neun Positionen (CS1 bis CS9) gemessen in DVT-Aufnahmen

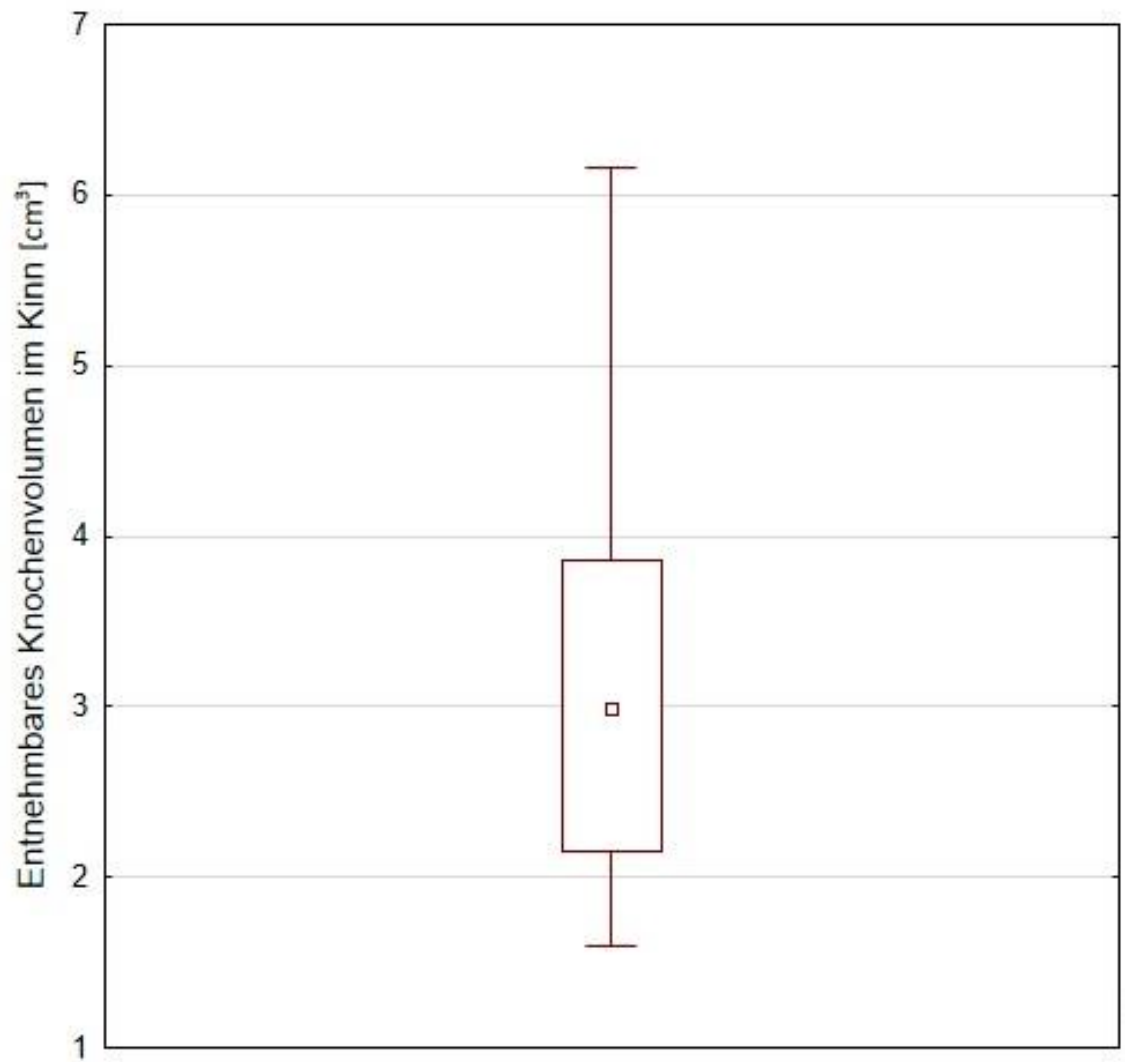

Abbildung 37 Box-Whisker-Plot des errechneten entnehmbaren Knochenvolumens $\left[\mathrm{cm}^{3}\right]$ im Kinn (Vchin) 


\subsubsection{DVT-Messungen der retromolaren Region}

Die mesio-distale Länge des potentiellen retromolaren Knochentransplantates wurde mit $20 \mathrm{~mm}$ festgelegt, da eine eindeutige distale Begrenzung durch sensible anatomische Strukturen wie z. B. Nerven nicht gegeben ist. Die vertikale Knochenhöhe von der okklusal gelegenen Knochenoberfläche bis zum Nervus alveolaris inferior wurde an drei Punkten gemessen (MN1 bis MN3). An jeder dieser drei vertikalen Strecken, wurde an jeweils drei Punkten die Knochendicke in bukko-lingualer Richtung gemessen (MW1 bis MW9). Aus diesen Strecken konnte das Knochenvolumen des retromolaren Knochenblocks errechnet werden (Vretro). Eine schematische Darstellung ist der Abbildung 23 zu entnehmen.

Auf Höhe der halben Wurzellänge der zweiten Unterkiefermolaren wurde die Dicke der bukkalen Knochenwand bei beiden Wurzeln gemessen (MR1 und MR2). Abschließend wurde im Frontalschnitt beidseitig untersucht, ob der Nervus alveolaris inferior $5 \mathrm{~mm}$ distal des zweiten Molaren in der lingualen oder bukkalen Hälfte verläuft (Abb. 25). Folgende Tabelle fasst die deskriptive Statistik des retromolaren Areals zusammen.

Tabelle 6 Deskriptive Statistik der retromolaren Messungen im DVT. Vretro ist in $\left[\mathrm{cm}^{3}\right]$ angegeben. Die Parameter $\mathrm{MN}, \mathrm{MW}$ und $\mathrm{MR}$ sind in [mm] angegeben.

\begin{tabular}{|c|c|c|c|c|c|}
\hline Parameter & $\mathbf{n}$ & Mittelwert [mm] & Minimum [mm] & Maximum [mm] & SD [mm] \\
\hline MN1 & 36 & 11,09 & 5,55 & 16,60 & 2,63 \\
\hline MN2 & 36 & 10,26 & 4,95 & 16,50 & 3,12 \\
\hline MN3 & 36 & 12,30 & 7,05 & 22,00 & 3,90 \\
\hline MW1 & 36 & 7,85 & 3,45 & 14,00 & 2,43 \\
\hline MW2 & 36 & 10,66 & 6,00 & 14,75 & 2,10 \\
\hline MW3 & 36 & 8,55 & 3,15 & 12,20 & 1,90 \\
\hline MW4 & 36 & 6,40 & 1,50 & 11,40 & 2,59 \\
\hline MW5 & 36 & 9,50 & 5,50 & 13,95 & 2,17 \\
\hline MW6 & 36 & 7,89 & 3,10 & 10,80 & 2,01 \\
\hline MW7 & 36 & 2,74 & 0,20 & 10,40 & 2,16 \\
\hline MW8 & 36 & 6,46 & 2,65 & 12,00 & 1,97 \\
\hline MW9 & 36 & 6,36 & 2,95 & 10,35 & 1,43 \\
\hline MR1 & 36 & 3,14 & 1,05 & 7,35 & 1,83 \\
\hline MR2 & 36 & 4,49 & 1,20 & 0,54 \\
\hline Vretro [cm 3 ] & 36 & 1,66 & 0,69 & 2,00 & 2 \\
\hline
\end{tabular}


Die durchschnittliche Entfernung zwischen der okklusal gelegenen Knochenoberfläche und dem Nervus alveolaris inferior ist bei der distalsten Messposition am größten (MN3). Die vestibuläre Knochenwand ist bei der distalen Wurzel (MR2) durchschnittlich dicker als bei der mesialen Wurzel (MR1) des zweiten Unterkiefermolaren. Die durchschnittliche bukkolinguale Knochendicke der mesialen Messpunkte (MW1 bis MW3) ist größer als jene der distalen (MW4 bis MW6 bzw. MW7 bis MW9). Außerdem bestehen Differenzen in kraniokaudaler Richtung. So ist die durchschnittliche bukko-linguale Knochendicke auf halber Länge zwischen Knochenoberfläche und Nerv (MW2, MW5, MW8) größer, als in den kranialen (MW1, MW4, MW7) und kaudalen Messpositionen (MW3, MW6, MW9). Für das retromolare Gebiet wurde ein durchschnittliches Knochenvolumen von 1,66 $\mathrm{cm}^{3}$ mit einer Standardabweichung von $0,54 \mathrm{~cm}^{3}$ gemessen.

Der Nervus alveolaris inferior verläuft in der untersuchten Population doppelt so häufig entlang der lingualen Hälfte wie in der bukkalen. Der Nerv verlief in der untersuchten Patientenpopulation hierbei symmetrisch, sodass er auf beiden untersuchten Seiten entweder lingual oder bukkal verlief. Für die grafische Darstellung der Messergebnisse wurden BoxWhisker-Plots erstellt.

\begin{tabular}{|c|c|}
\hline Verlauf des Nervus alveolaris inferior & $\mathbf{n}$ \\
\hline Lingual & 24 \\
\hline Bukkal & 12 \\
\hline gesamt & 36 \\
\hline
\end{tabular}



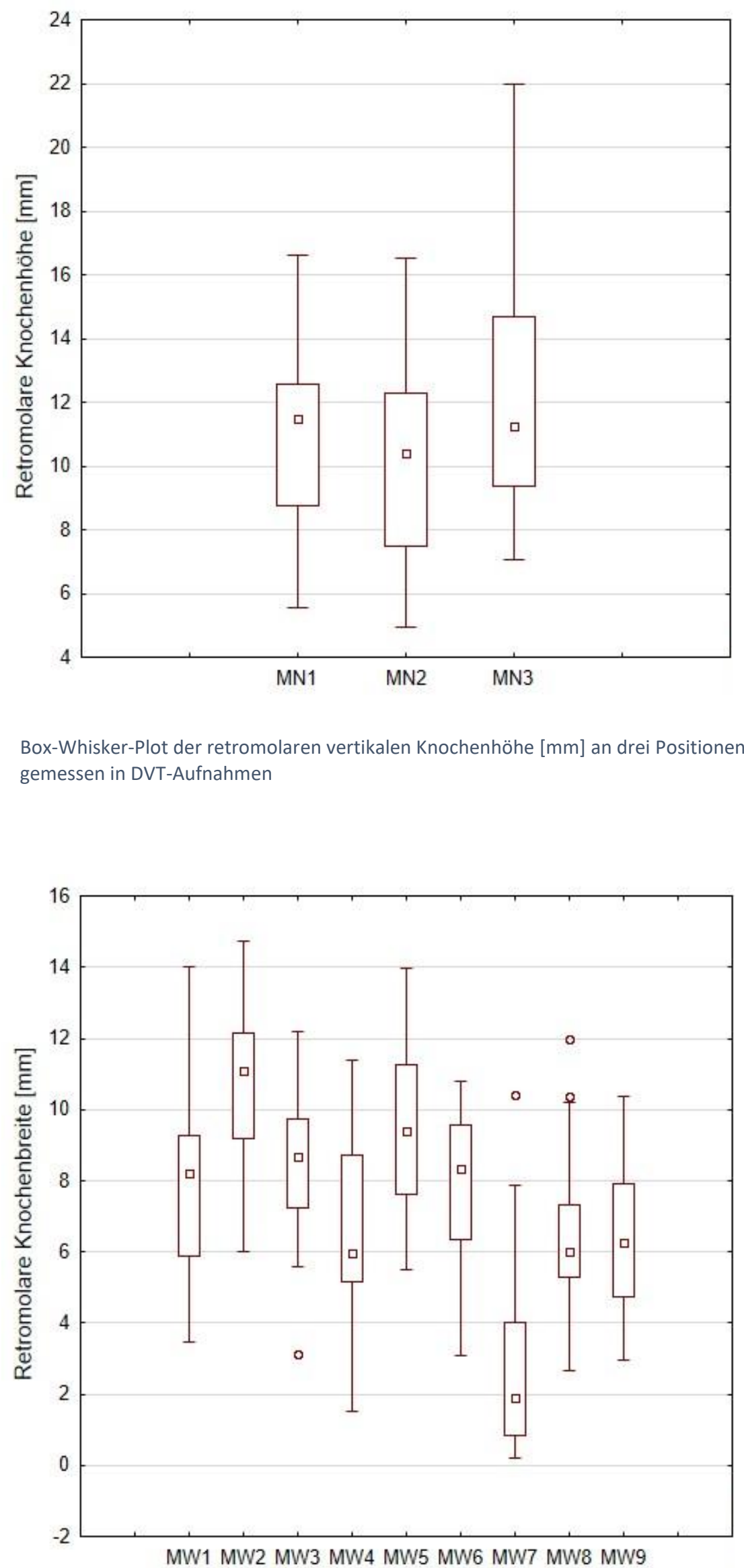


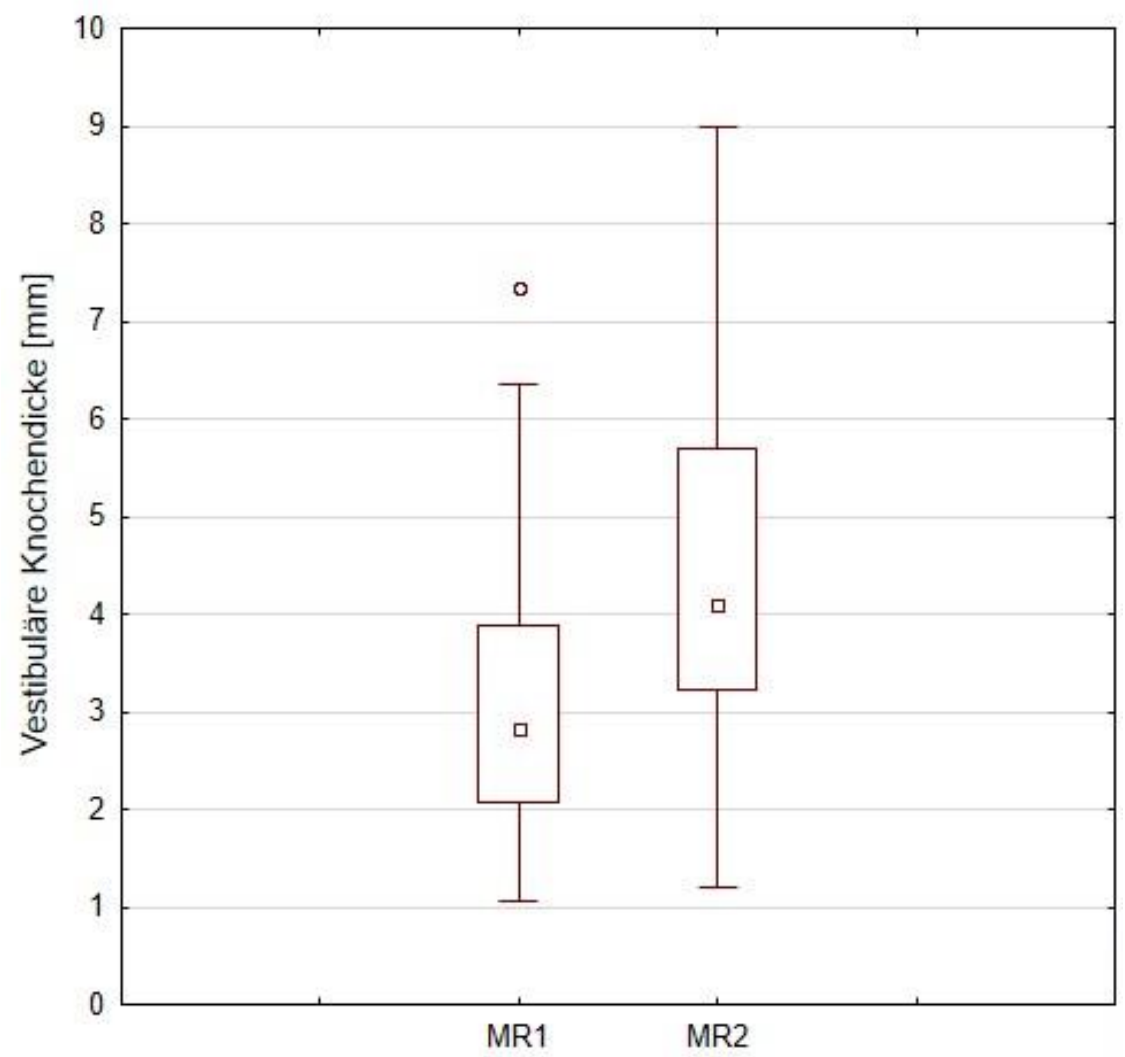

Abbildung 41

Box-Whisker-Plot der vestibulären Knochendicke [mm] an beiden Wurzeln des zweiten Unterkiefermolaren (MR1 und MR2) gemessen in DVT-Aufnahmen

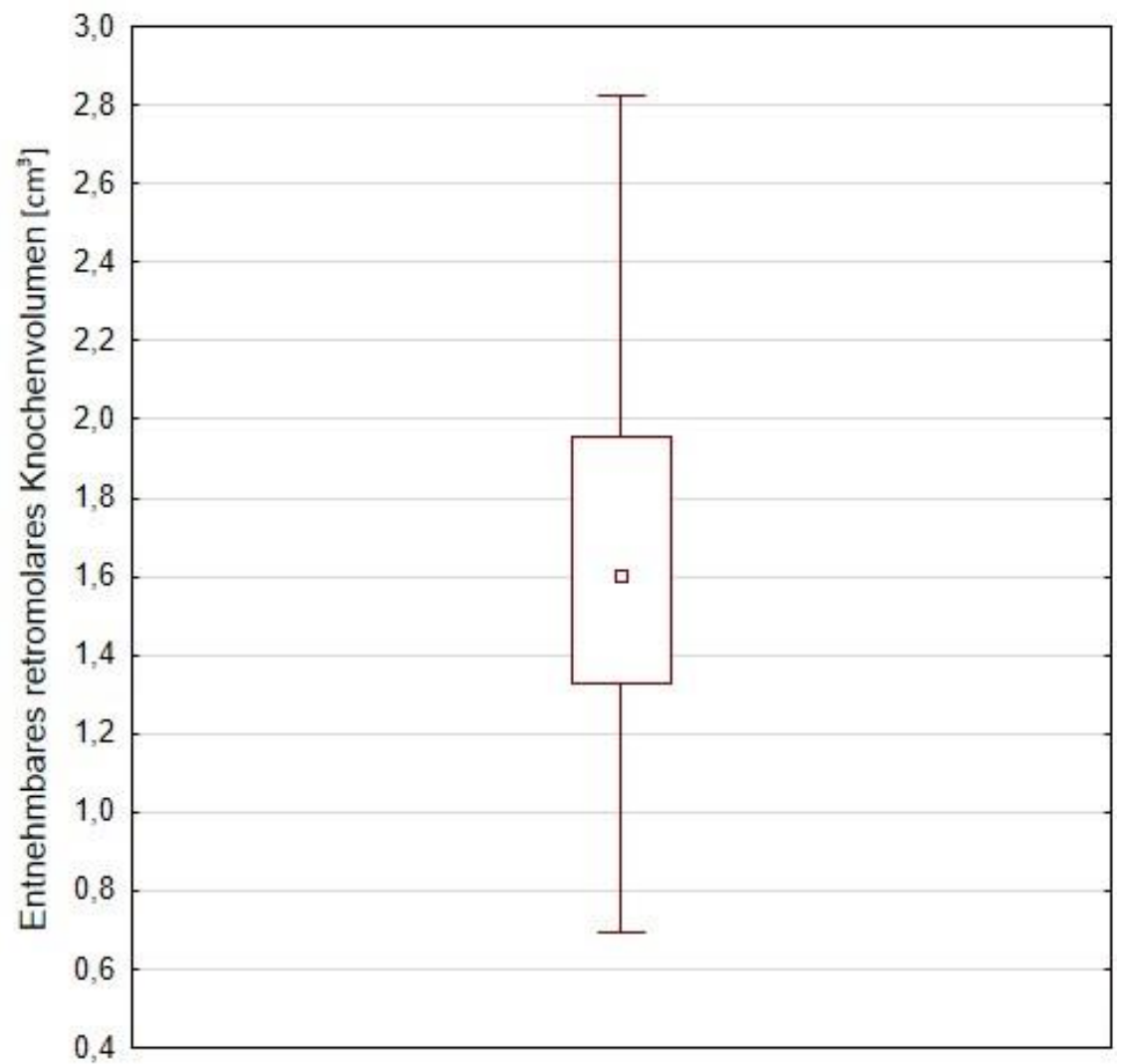

Abbildung 42

Box-Whisker-Plot des errechneten entnehmbaren retromolaren Knochenvolumens (Vretro) $\left[\mathrm{cm}^{3}\right]$ 


\subsubsection{DVT-Messungen der Crista zygomatico-alveolaris}

Da für das potentielle Knochentransplantat in Regio der Crista zygomatico-alveolaris vorab eine Höhe und Breite von jeweils 10 mm festgelegt wurde, war ausschließlich die Knochendicke an neun Messpositionen (ZA1 bis ZA9) zu bestimmen und das entsprechende Volumen (Vcrista) zu berechnen. Eine schematische Zeichnung der Messpositionen ist in Abbildung 21 dargestellt. Die deskriptive Statistik kann folgender Tabelle entnommen werden.

Tabelle 8 Deskriptive Statistik der DVT-Messungen an der Crista zygomatico-alveolaris. Vcrista ist in [ $\mathrm{cm}^{3}$ ] angegeben. Die Werte für die Knochendicke (ZA) sind in [mm] angegeben.

\begin{tabular}{|c|c|c|c|c|c|}
\hline Parameter & $\mathbf{n}$ & Mittelwert [mm] & Minimum [mm] & Maximum [mm] & SD [mm] \\
\hline ZA1 & 36 & 1,55 & 0,80 & 2,70 & 0,45 \\
\hline ZA2 & 36 & 1,38 & 0,70 & 2,45 & 0,41 \\
\hline ZA3 & 36 & 1,68 & 0,70 & 3,10 & 0,55 \\
\hline ZA4 & 36 & 2,06 & 0,95 & 4,10 & 0,77 \\
\hline ZA5 & 36 & 1,63 & 0,80 & 3,00 & 0,53 \\
\hline ZA6 & 36 & 2,08 & 1,00 & 3,55 & 0,57 \\
\hline ZA7 & 36 & 1,49 & 0,60 & 3,00 & 0,53 \\
\hline ZA8 & 36 & 1,19 & 0,55 & 2,60 & 0,43 \\
\hline ZA9 & 36 & 1,80 & 0,65 & 3,15 & 0,61 \\
\hline Vcrista $\left[\mathrm{cm}^{3}\right]$ & 36 & 0,17 & 0,10 & 0,25 & 0,04 \\
\hline
\end{tabular}

Die Messpositionen auf dem Wendepunkt der Crista zygomatico-alveolaris (ZA4 bis ZA6) weisen durchschnittlich größere Knochendicken auf als die lateral davon befindlichen (ZA1 bis ZA3 bzw. ZA7 bis ZA9). Mit durchschnittlich 0,17 $\mathrm{cm}^{3}$, maximal $0,25 \mathrm{~cm}^{3}$ und einer Standardabweichung von $0,04 \mathrm{~cm}^{3}$ liegt bei der Crista zygomatico-alveolaris das geringste Knochenvolumen unter den untersuchten Entnahmeregionen vor. Box-Whisker-Plots stellen die Messergebnisse grafisch dar. 


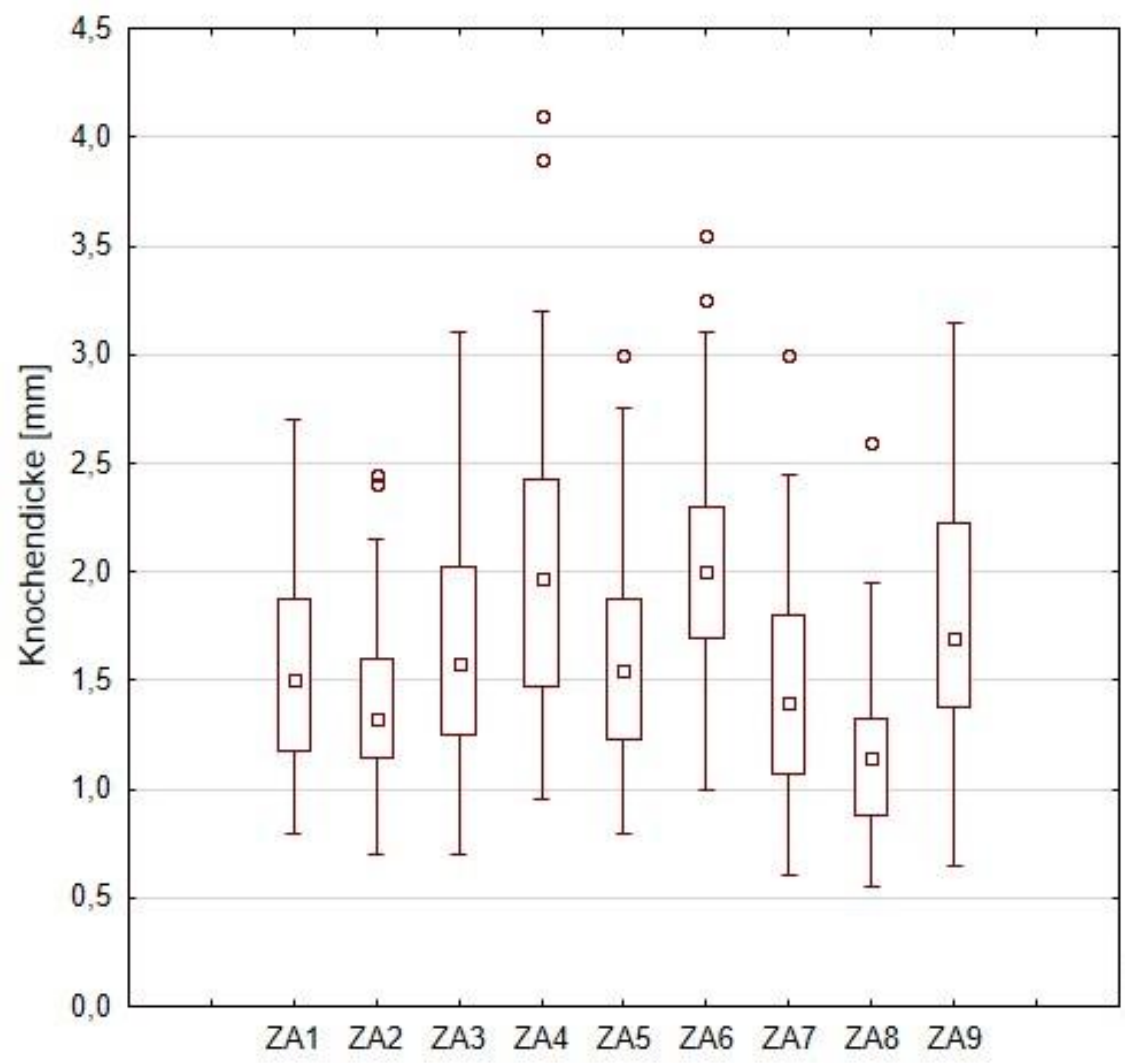

Abbildung 44

Box-Whisker-Plot der Knochendicke [mm] an neun Messpositionen der Crista zygomatico-alveolaris (ZA1 bis ZA9) gemessen in DVT-Aufnahmen

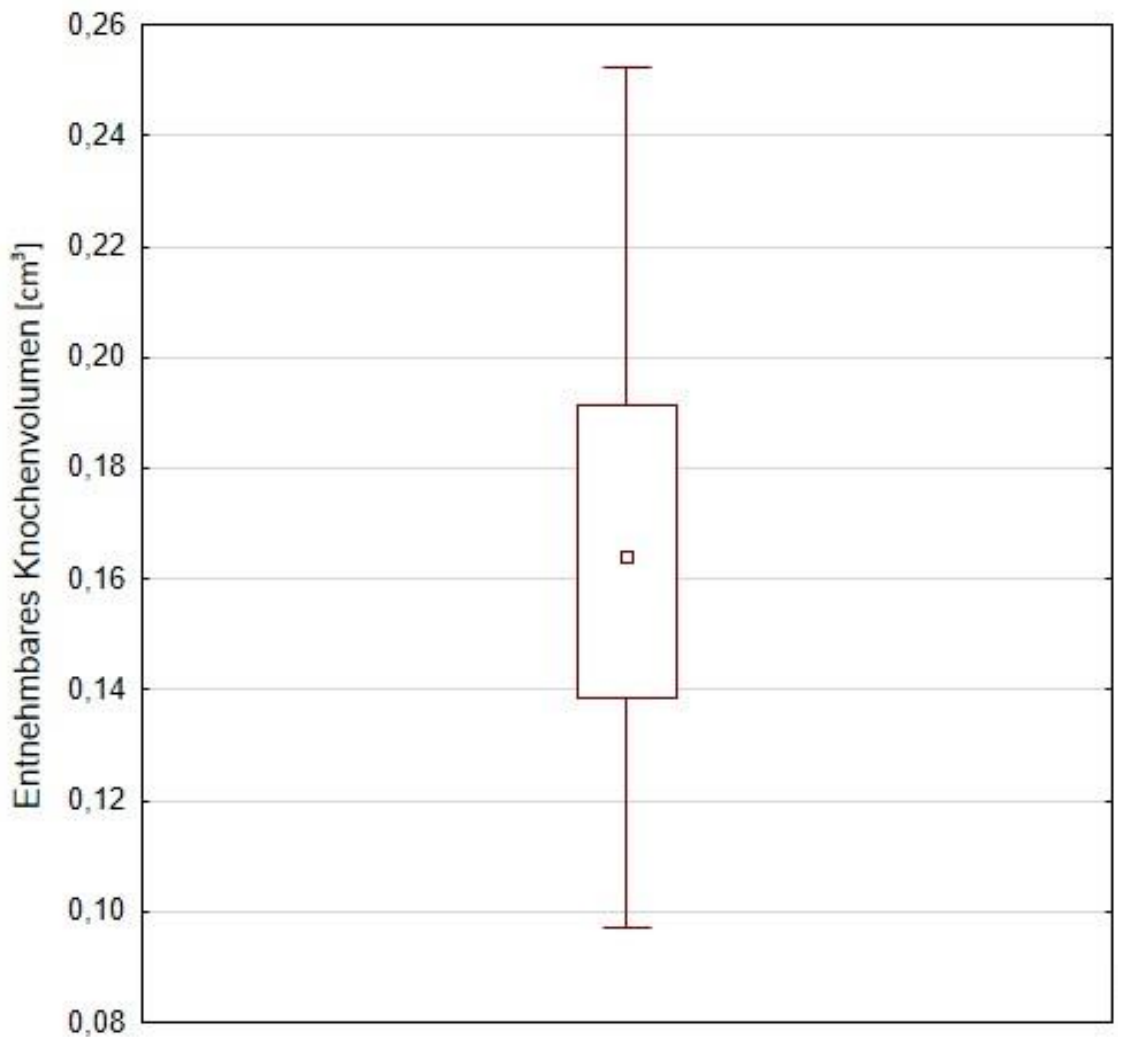

Box-Whisker-Plot des errechneten entnehmbaren Knochenvolumens $\left[\mathrm{cm}^{3}\right]$ an der Crista zygomatico-alveolaris (Vcrista) 


\subsection{Inter-Rater Reliabilität}

Bevor nach Korrelationen zwischen den Messergebnissen der Röntgenverfahren gesucht werden kann, sollte die Reliabilität der Messungen geprüft werden. Hierfür wurde ein weiterer Rater beauftragt, anhand der erläuterten Methoden die Messungen an den gleichen Röntgenaufnahmen durchzuführen. Anschließend wurde die Inter-Rater Reliabilität mit Hilfe von Bland-Altman-Plots untersucht.

Vor der Anwendung des Bland-Altman-Plots müssen die Messdifferenzen beider Rater auf eine Normalverteilung geprüft werden. Dies erfolgt mit Hilfe von Shapiro-Wilk-Tests, von denen einer exemplarisch dargestellt wird.

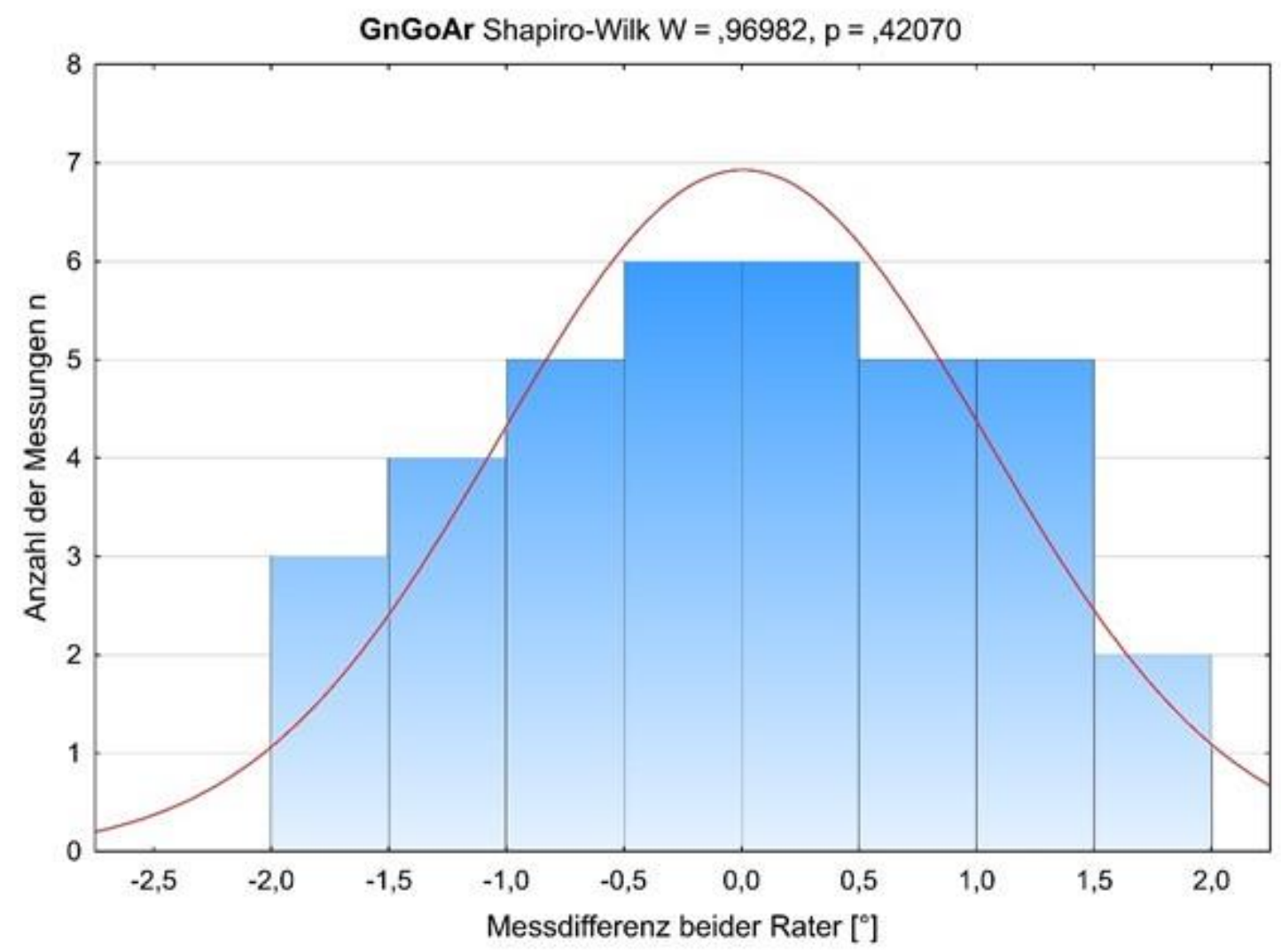


Bei dem Shapiro-Wilk-Test nimmt die Nullhypothese an, dass eine Normalverteilung vorliegt. Da der $p$-Wert mit $p=0,42$ deutlich über 0,05 lag, wird die Nullhypothese nicht abgelehnt. Deshalb wird die Normalverteilung angenommen und ein Bland-Altman-Plot erstellt. Ein Plot wird zur Veranschaulichung dargestellt und erläutert.

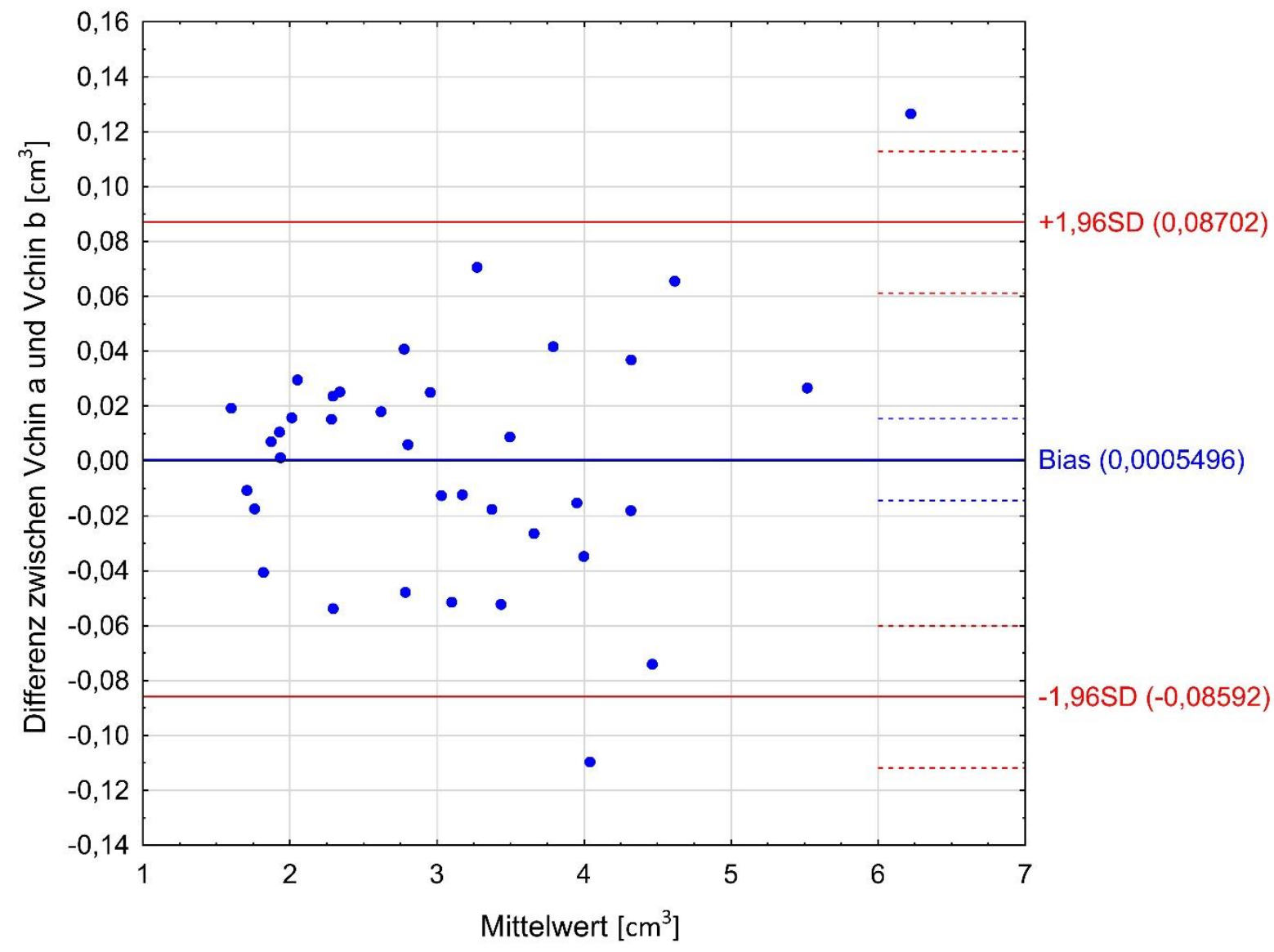
Kinn (Vchin) $\left[\mathrm{cm}^{3}\right]$ (Vchin $\mathrm{a}=$ Knochenvolumen gemessen durch Rater $\mathrm{a}$, Vchin $\mathrm{b}=$ Knochenvolumen gemessen durch Rater b)

Auf der X-Achse des Plots sind die Mittelwerte der Messungen beider Rater dargestellt, während die Y-Achse die Differenz zwischen diesen Messungen abbildet. Diese Differenzen bilden zusammen einen Mittelwert (Bias), aus dessen Standardabweichung zwei „Limits of Agreement" erstellt werden:

Ein oberes Limit of Agreement (LoA+), hier durch die obere rote Waagerechte markiert und ein unteres Limit of Agreement (LoA-), hier durch die untere rote Waagerechte markiert. 
Folgende Aussage bezüglich der Interrater-Differenz für Vchin darf getroffen werden:

$95 \%$ der Messdifferenzen zwischen Rater a und Rater b liegen zwischen dem LoA- und LoA+ und somit in einem Bereich von $-0,086 \mathrm{~cm}^{3}$ bis $0,087 \mathrm{~cm}^{3}$.

Die Limits of Agreement für die restlichen Messungen werden tabellarisch dargestellt.

\begin{tabular}{|c|c|c|}
\hline Parameter & Lower Limit of Agreement & Upper Limit of Agreement \\
\hline Vchin $\left[\mathrm{cm}^{3}\right]$ & $-0,086$ & 0,087 \\
\hline Vretro $\left[\mathrm{cm}^{3}\right]$ & $-0,042$ & 0,039 \\
\hline Vcrista $\left[\mathrm{cm}^{3}\right]$ & $-0,0052$ & 0,0097 \\
\hline WS1 [mm] & $-0,22$ & 0,27 \\
\hline WS7 [mm] & $-0,27$ & 0,24 \\
\hline Ang. Mand. $\left[{ }^{\circ}\right]$ & $-1,85$ & 1,89 \\
\hline Hasund [Punkte] & $-1,39$ & 1,34 \\
\hline SNA $\left[{ }^{\circ}\right]$ & $-1,14$ & 1,04 \\
\hline SNB $\left[{ }^{\circ}\right]$ & $-1,51$ & 2,41 \\
\hline ANB $\left[^{\circ}\right]$ & $-3,25$ & 2,49 \\
\hline $\mathrm{ML}-\mathrm{NSL}\left[{ }^{\circ}\right]$ & $-1,55$ & 1,78 \\
\hline NL-NSL $\left[{ }^{\circ}\right]$ & $-0,92$ & 1,19 \\
\hline $\mathrm{ML}-\mathrm{NL}\left[{ }^{\circ}\right]$ & $-1,44$ & 1,4 \\
\hline $\mathrm{NSBa}\left[{ }^{\circ}\right]$ & $-3,91$ & 3,44 \\
\hline Index [\%] & $-2,31$ & 1,84 \\
\hline Gn-Go-Ar $\left[^{\circ}\right]$ & $-2,06$ & 2,04 \\
\hline
\end{tabular}

Die Tabelle lässt sich ähnlich wie der obige Plot ablesen. Für die Messung WS1 befinden sich $95 \%$ der Messdifferenzen zwischen beiden Ratern in einem Bereich von -0,22 mm und 0,27 mm. Für den Winkel ML-NSL liegen 95 \% der Messdifferenzen in einem Bereich von $-1,55^{\circ}$ und $1,78^{\circ}$. 


\subsection{Korrelationen zwischen Messwerten}

\subsubsection{Untersuchung auf Normalverteilung}

Um die Pearson-Korrelationen zwischen den erhobenen Daten bestimmen zu können, müssen diese zuvor auf eine Normalverteilung geprüft werden. Hierzu kommen Shapiro-WilkTests zur Anwendung.

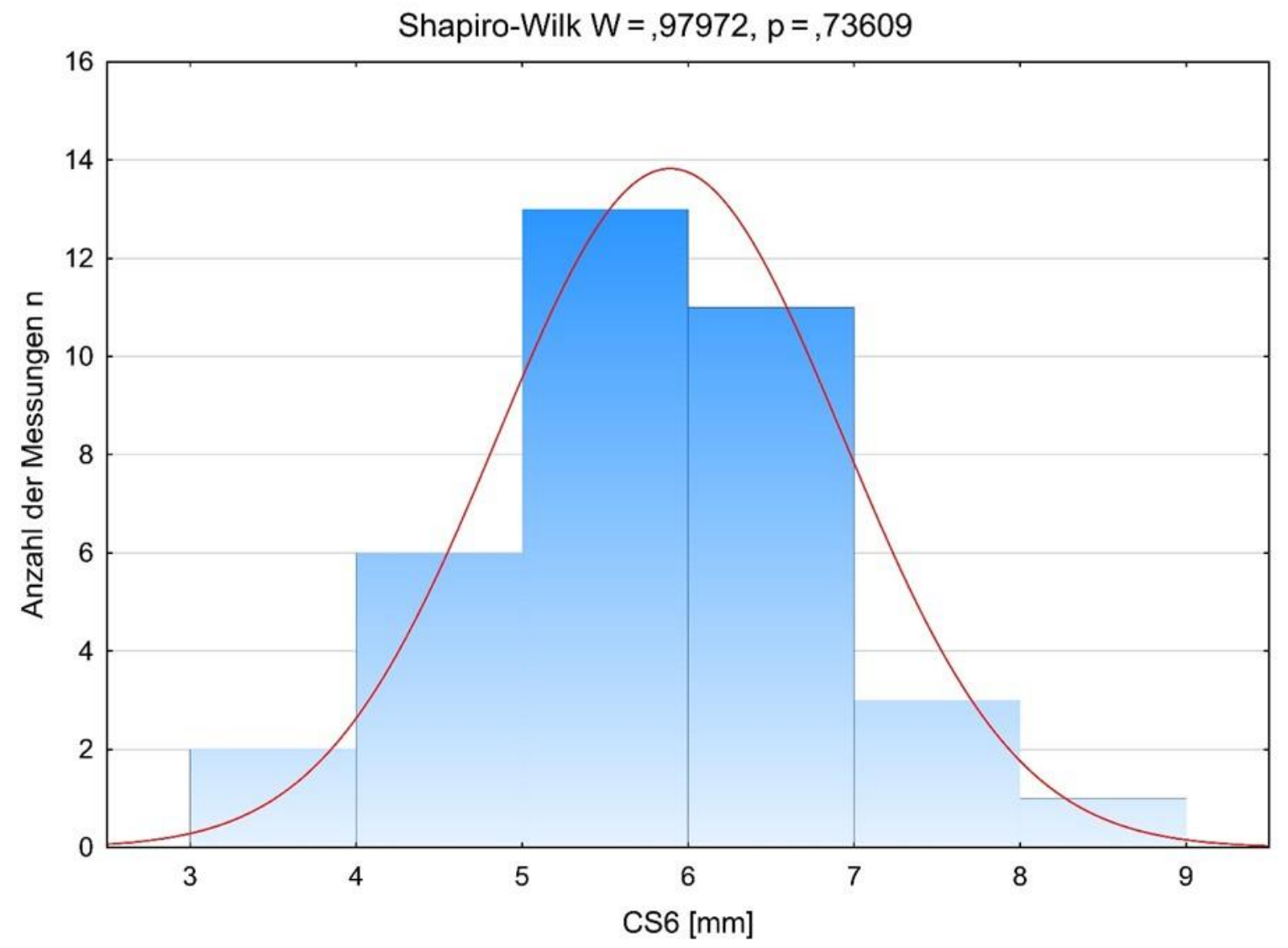

Abbildung 47

Shapiro-Wilk-Test auf Normalverteilung für die sagittale Knochendicke [mm] im Kinn (CS6), gemessen in DVT-Aufnahmen

Die Nullhypothese mit der Annahme, dass die Werte normalverteilt sind, wird aufgrund des hohen p-Wertes von 0,74 nicht abgelehnt. Die Normalverteilung wird daher angenommen. Diese Prüfung erfolgte für sämtliche Messungen im OPG, FRS und DVT. Eine Normalverteilung kann bei allen Messungen angenommen werden. 


\subsubsection{Einfluss des Geschlechts}

Aufgrund der binären Verteilung des Geschlechtes wird vorab ein t-test durchgeführt, um signifikante Unterschiede zwischen den beiden Gruppen in Bezug auf die kephalometrischen Messungen aufzudecken.

Tabelle 10 T-test für die Untersuchung des Geschlechts als unabhängige und $\mathrm{CH} 2 \mathrm{bzw}$. CH3 [mm] als abhängige Variable

\begin{tabular}{|c|c|c|c|c|c|}
\hline Variable & $\mathbf{n}$ weiblich & $\mathbf{n}$ männlich & $\begin{array}{c}\text { Mittelwert } \\
\text { weiblich } \\
{[\mathbf{m m}]}\end{array}$ & $\begin{array}{c}\text { Mittelwert } \\
\text { männlich }\end{array}$ & p-Wert \\
\hline $\mathrm{CH} 2$ & 20 & 16 & 41,49 & 44,93 & 0,0359 \\
\hline $\mathrm{CH} 3$ & 20 & 16 & 36,84 & 42,99 & 0,0026 \\
\hline
\end{tabular}

Die horizontale Breite des knöchernen Kinns weist auf der Höhe des Pogonions $(\mathrm{CH} 2)$ und $2 \mathrm{~mm}$ kranial von dem Unterkieferrand ( $\mathrm{CH} 3$ ) statistisch signifikante Unterschiede zwischen männlichen und weiblichen Patienten auf. Um den Einfluss des Geschlechts auf diese beiden Parameter genauer zu quantifizieren, werden univariate lineare Regressionsmodelle erstellt. Hierbei fungiert das Geschlecht als unabhängige Variable und $\mathrm{CH} 2$ bzw. $\mathrm{CH} 3$ als abhängige Variable.

Tabelle 11 Univariate lineare Regression mit dem Geschlecht als unabhängige Variable und $\mathrm{CH} 2$ als abhängige Variable

\begin{tabular}{|c|c|c|c|c|}
\hline $\mathbf{n}$ & $\mathbf{b}$ & SE b & $\mathbf{t ~ ( 3 4 )}$ & $\mathbf{p}-$ Wert \\
\hline 36 & $-3,44$ & 1,57 & $-2,19$ & 0,036 \\
\hline
\end{tabular}

$\mathrm{n}=$ Anzahl untersuchter Patienten, $\mathrm{b}=$ Regressions-koeffizient, $\mathrm{SE} \mathrm{b}=$ Standardfehler für $\mathrm{b}, \mathrm{t}=$ Quotient aus $\mathrm{b}$ und SE $\mathrm{b}$

Das Ergebnis der Regressionsmodelle zeigt, dass das Geschlecht in der untersuchten Population einen signifikanten Einfluss auf die horizontale Breite des knöchernen Kinns auf Höhe des Pogonions ( $\mathrm{CH} 2)$ hat. Hier liegt bei dem weiblichen Geschlecht eine durchschnittlich 3,44 mm kleinere Breite, als bei dem männlichen Geschlecht vor. 
Ein entsprechendes Regressionsmodell wurde auch für $\mathrm{CH} 3$ erstellt.

Tabelle 12 Univariate lineare Regression mit dem Geschlecht als unabhängige Variable und CH3 als abhängige Variable

\begin{tabular}{|c|c|c|c|c|}
\hline $\mathbf{n}$ & $\mathbf{b}$ & SE b & $\mathbf{t}(\mathbf{3 4 )}$ & $\mathbf{p}-$ Wert \\
\hline 36 & $-6,15$ & 1,89 & $-3,25$ & 0,002 \\
\hline
\end{tabular}

$\mathrm{n}=$ Anzahl untersuchter Patienten, $\mathrm{b}=$ Regressions-koeffizient, $\mathrm{SE} \mathrm{b}=$ Standardfehler für $\mathrm{b}, \mathrm{t}=$ Quotient aus $\mathrm{b}$ und SE $\mathrm{b}$

Auch hier kann aufgrund des Regressionsmodells die Aussage getroffen werden, dass das Geschlecht in der untersuchten Population einen signifikanten Einfluss auf die horizontale Breite des knöchernen Kinns in einem Abstand von $2 \mathrm{~mm}$ zur Unterkieferbasis hat (CH3). Im Vergleich zum männlichen, ist beim weiblichen Geschlecht diese Breite durchschnittlich $6,15 \mathrm{~mm}$ kleiner.

Statistisch signifikante Unterschiede zwischen den Geschlechtern bezüglich der Knochenvolumina der drei Entnahmeregionen konnten im t-test nicht nachgewiesen werden.

Tabelle $13 \quad$ T-test für die Untersuchung des Geschlechts als unabhängige und die Knochenvolumina $\left[\mathrm{cm}^{3}\right]$ als abhängige Variable

\begin{tabular}{|c|c|c|c|c|c|}
\hline Variable & $\mathbf{n}$ weiblich & $\mathbf{n}$ männlich & $\begin{array}{c}\text { Mittelwert } \\
\text { weiblich } \\
{\left[\mathbf{c m}^{\mathbf{3}}\right]}\end{array}$ & $\begin{array}{c}\text { Mittelwert } \\
\text { männlich } \\
{\left[\mathbf{c m}^{\mathbf{3}}\right]}\end{array}$ & $\mathbf{p - W e r t}$ \\
\hline Vchin & 20 & 16 & 2,85 & 3,41 & 0,13 \\
\hline Vretro & 20 & 16 & 1,68 & 1,63 & 0,77 \\
\hline Vcrista & 20 & 16 & 0,17 & 0,15 & 0,14 \\
\hline
\end{tabular}


Eine grafische Darstellung der durchschnittlichen Knochenvolumina im Zusammenhang mit dem Geschlecht wurde als Box-Whisker-Plot wurde angefertigt.

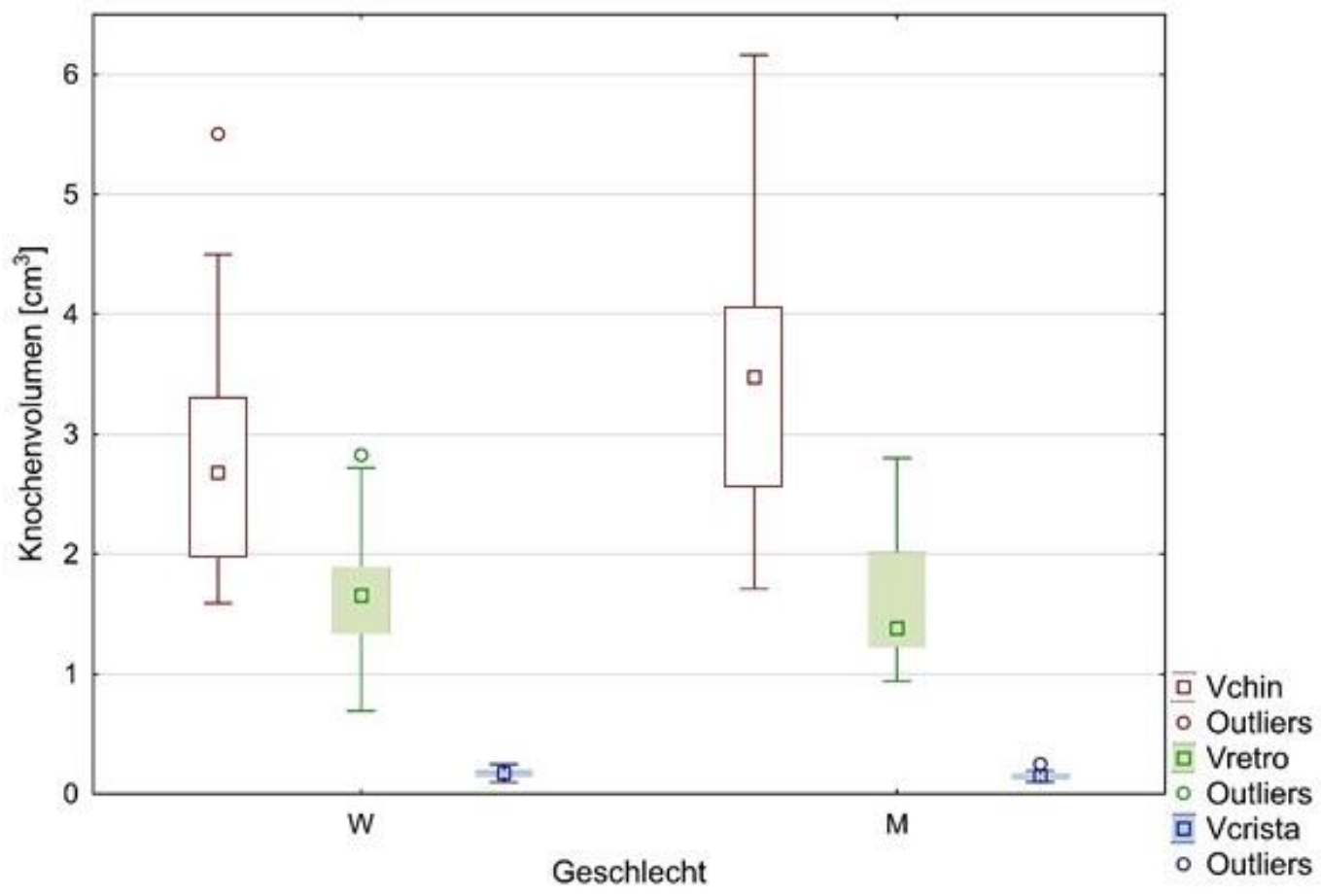

Abbildung 48 Box-Whisker-Plot der Knochenvolumina $\left[\mathrm{cm}^{3}\right]$ verschlüsselt nach Geschlecht und Entnahmeareal

Neben den kephalometrischen Messungen der Tabelle 9 wurde auch der bukko-linguale Verlauf des Nervus alveolaris inferior (Abb. 25) auf eine mögliche Abhängigkeit vom Geschlecht untersucht. Da beide Variablen binär codiert sind, wurde ein Chi-Quadrat-Test durchgeführt.

Tabelle 14 Tabelle zum Chi-Quadrat-Test zur Prüfung einer möglichen Abhängigkeit des Nervverlaufs von dem Geschlecht

\begin{tabular}{|c|c|c|c|}
\hline Statistik & Chi-Quadrat & df & p-Wert \\
\hline Pearson Chi-Quadrat & 0,9 & 1 & 0,34 \\
\hline
\end{tabular}

$\mathrm{df}=$ Freiheitsgrade

Aufgrund des hohen p-Wertes von 0,34 ist keine Abhängigkeit des Nervverlaufs von dem Geschlecht zu erwarten. 


\subsubsection{Zusammenhang zwischen kephalometrischen Parametern und Nervverlauf}

Neben dem Geschlecht als möglichen Einflussfaktor auf den Verlauf des Nervus alveolaris inferior, wurden zusätzlich die kephalometrischen Messungen aus OPG, FRS und DVT auf ihre möglichen Zusammenhänge hin untersucht.

Tabelle 15 T-tests für die Untersuchung der kephalometrischen Messungen als unabhängige Variable und den Nervverlauf als abhängige Variable. Es sind lediglich die statistisch signifikanten Parameter mit $p=<0,05$ dargestellt.

\begin{tabular}{|c|c|c|c|c|c|}
\hline Parameter & $\begin{array}{c}\text { Mittelwert bei bukkalem } \\
\text { Nervverlauf }\end{array}$ & $\begin{array}{c}\text { Mittelwert bei lingualem } \\
\text { Nervverlauf }\end{array}$ & $\begin{array}{c}\text { p- } \\
\text { Wert }\end{array}$ & $\mathbf{n}$ bukkal & n lingual \\
\hline NSBa $\left[^{\circ}\right.$ ] & 136,36 & 129,65 & 0,039 & 12 & 24 \\
\hline Gn-Go-Ar $\left[^{\circ}\right]$ & 119,65 & 128,18 & 0,003 & 12 & 24 \\
\hline Ang. Mand. ${ }^{\circ}{ }^{\circ}$ & 123,25 & 129,62 & 0,010 & 12 & 24 \\
\hline CS1 $[\mathrm{mm}]$ & 7,85 & 6,42 & 0,027 & 12 & 24 \\
\hline Hasund [Punkte] & 6,83 & 0,29 & 0,006 & 12 & 24 \\
\hline
\end{tabular}

Die Parameter NSBa, Gn-Go-Ar, Ang. Mand., CS1 und Hasund weisen laut t-test einen Zusammenhang mit dem Nervverlauf auf. Der durchschnittliche Kieferwinkel, gemessen im FRS und OPG, ist bei lingualem Verlauf des Nerven höher als bei bukkalem. Bei lingualem Nervverlauf ist die durchschnittliche sagittale Knochendicke des Kinns in der Position CS1 um 1,43 mm reduziert. Zudem ist der durchschnittliche Hasund-Score bei lingualem Nervverlauf um 6,54 Punkte reduziert und daher mit einer komparativ posterioren Rotation der Mandibula assoziiert.

Diese Parameter werden in das Modell für eine logistische Regression mit backward removal Algorithmus aufgenommen. Mit Hilfe dieses Algorithmus kann geprüft werden, welchen Einfluss diese Parameter haben. Bezüglich des Nervverlaufs in bukkaler Position ergaben sich folgende Odds Ratios.

Tabelle $16 \quad$ Logistische Regression mit backward removal Algorithmus zur Untersuchung des Einflusses kephalometrischer Parameter auf den Nervverlauf in bukkaler Position

\begin{tabular}{|c|c|c|}
\hline Parameter & Odds Ratio & p-Wert \\
\hline Hasund & 1,186 & 0,020 \\
\hline
\end{tabular}

Für einen bukkalen Nervverlauf liegt für den Hasund-Score eine Odds Ratio von 1,186 vor. Das Risiko, dass sich der Nerv an untersuchter Position in der bukkalen Hälfte der Mandibula befindet, erhöht sich um 18,6 \%, wenn der Hasund-Score um 1 erhöht wird. 
3.3.4 Korrelationen zwischen OPG-, FRS- und DVT-Messerwerten

Sämtliche Messungen in OPG-, FRS- und DVT-Aufnahmen wurden auf Korrelationen miteinander untersucht. Die Darstellung der Ergebnisse in diesem Abschnitt erfolgt getrennt nach den drei potentiellen Entnahmeregionen: Kinn, retromolare Region und Crista zygomatico-alveolaris. Zur Anwendung kommen hierbei Scatterplots und Tabellen.

\subsubsection{Korrelationen mit DVT-Messwerten im Kinn}

\begin{tabular}{|c|c|c|c|}
\hline \multicolumn{4}{|c|}{ Korrelationen zwischen FRS-Messungen und DVT-Messungen im Kinn } \\
\hline FRS Messung & DVT Messung (Kinn) & Korrelationskoeffizient & p-Wert \\
\hline \multirow[t]{7}{*}{ ML-NL } & $\mathrm{CH} 1$ & $-0,34$ & 0,042 \\
\hline & $\mathrm{CH} 2$ & $-0,44$ & 0,007 \\
\hline & $\mathrm{CH} 3$ & $-0,59$ & 0,000 \\
\hline & CV1 & 0,44 & 0,008 \\
\hline & $\mathrm{CV} 2$ & 0,43 & 0,009 \\
\hline & CV3 & 0,49 & 0,002 \\
\hline & CS4 & $-0,44$ & 0,007 \\
\hline \multirow[t]{7}{*}{ ML-NSL } & $\mathrm{CH} 1$ & $-0,36$ & 0,033 \\
\hline & $\mathrm{CH} 2$ & $-0,50$ & 0,002 \\
\hline & $\mathrm{CH} 3$ & $-0,66$ & 0,000 \\
\hline & CV1 & 0,45 & 0,006 \\
\hline & CV2 & 0,43 & 0,009 \\
\hline & CV3 & 0,48 & 0,003 \\
\hline & CS4 & $-0,50$ & 0,002 \\
\hline \multirow[t]{5}{*}{ Hasund } & $\mathrm{CH} 3$ & 0,53 & 0,001 \\
\hline & CV1 & $-0,44$ & 0,007 \\
\hline & $\mathrm{CV} 2$ & $-0,43$ & 0,009 \\
\hline & CV3 & $-0,47$ & 0,004 \\
\hline & CS4 & 0,46 & 0,005 \\
\hline ANB & CV3 & 0,43 & 0,009 \\
\hline Gn-Go-Ar & $\mathrm{CH} 3$ & $-0,41$ & 0,012 \\
\hline \multirow[t]{3}{*}{ Index } & CV1 & $-0,46$ & 0,005 \\
\hline & $\mathrm{CV} 2$ & $-0,42$ & 0,011 \\
\hline & CV3 & $-0,41$ & 0,014 \\
\hline
\end{tabular}


Kephalometrische Messungen an FRS-Aufnahmen helfen dabei Wachstumstendenzen und -typen zu diagnostizieren. Messwerte, die solche Wachstumsmuster beschreiben, korrelieren mit Knochendimensionen des Kinns die in den DVT-Aufnahmen gemessen wurden. Hierbei wird deutlich, dass FRS-Werte die offene/vertikale Wachstumstypen und posteriore Rotationen beschreiben, mit einem erhöhten vertikalen und verringerten sagittalen und transversalen Knochenangebot im Kinn statistisch signifikant korrelieren.

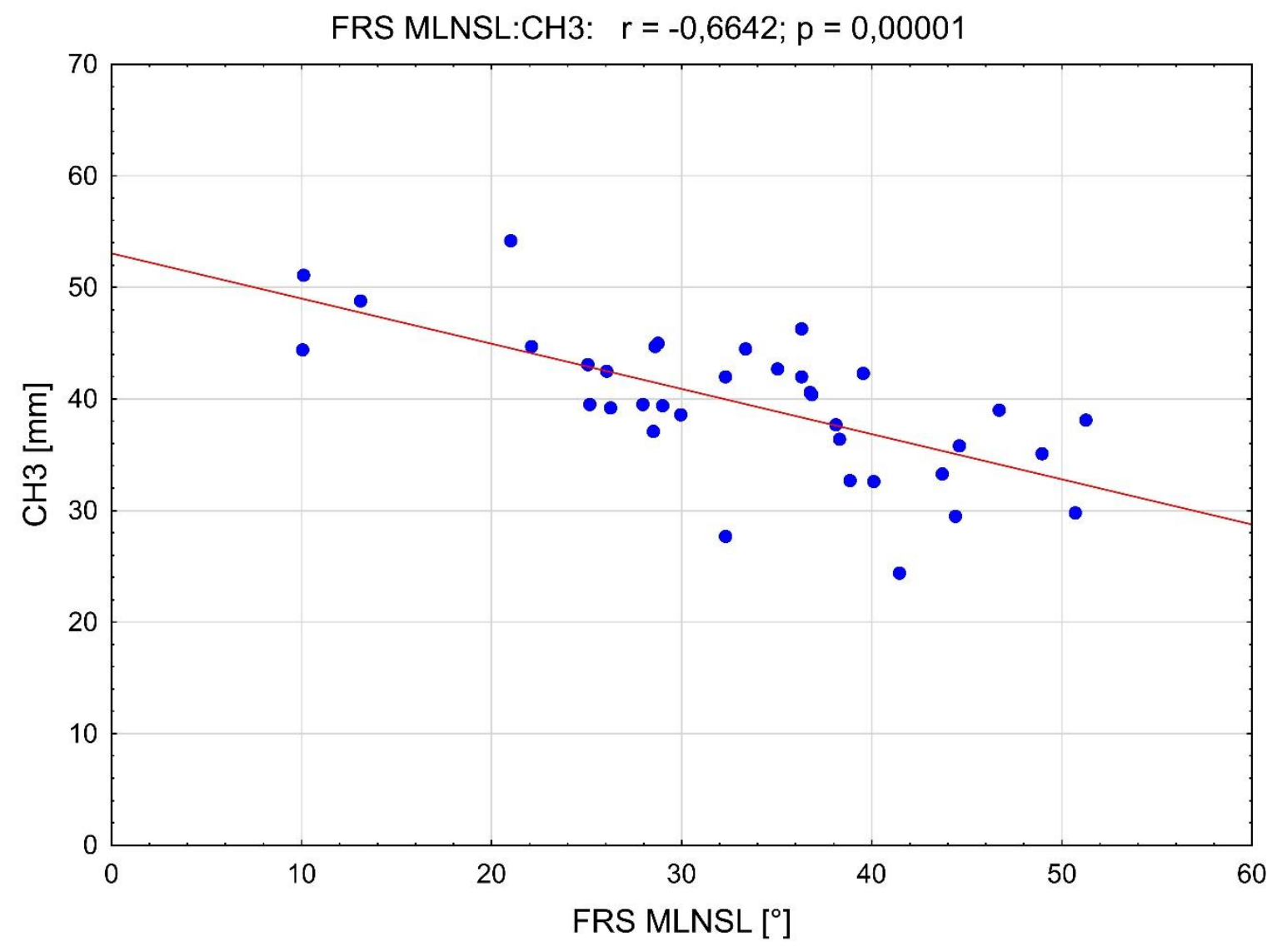

Mit einem Korrelationskoeffizienten von $r=-0,66$ und einem $p$-Wert von 0,00001 besteht eine statistisch signifikante negative Korrelation zwischen dem Winkel ML-NSL und der in der DVT-Aufnahme gemessenen Strecke CH3. Ein größerer ML-NSL korreliert also mit einem transversal schmaleren Kinn und somit reduziertem interforaminalem Abstand. 


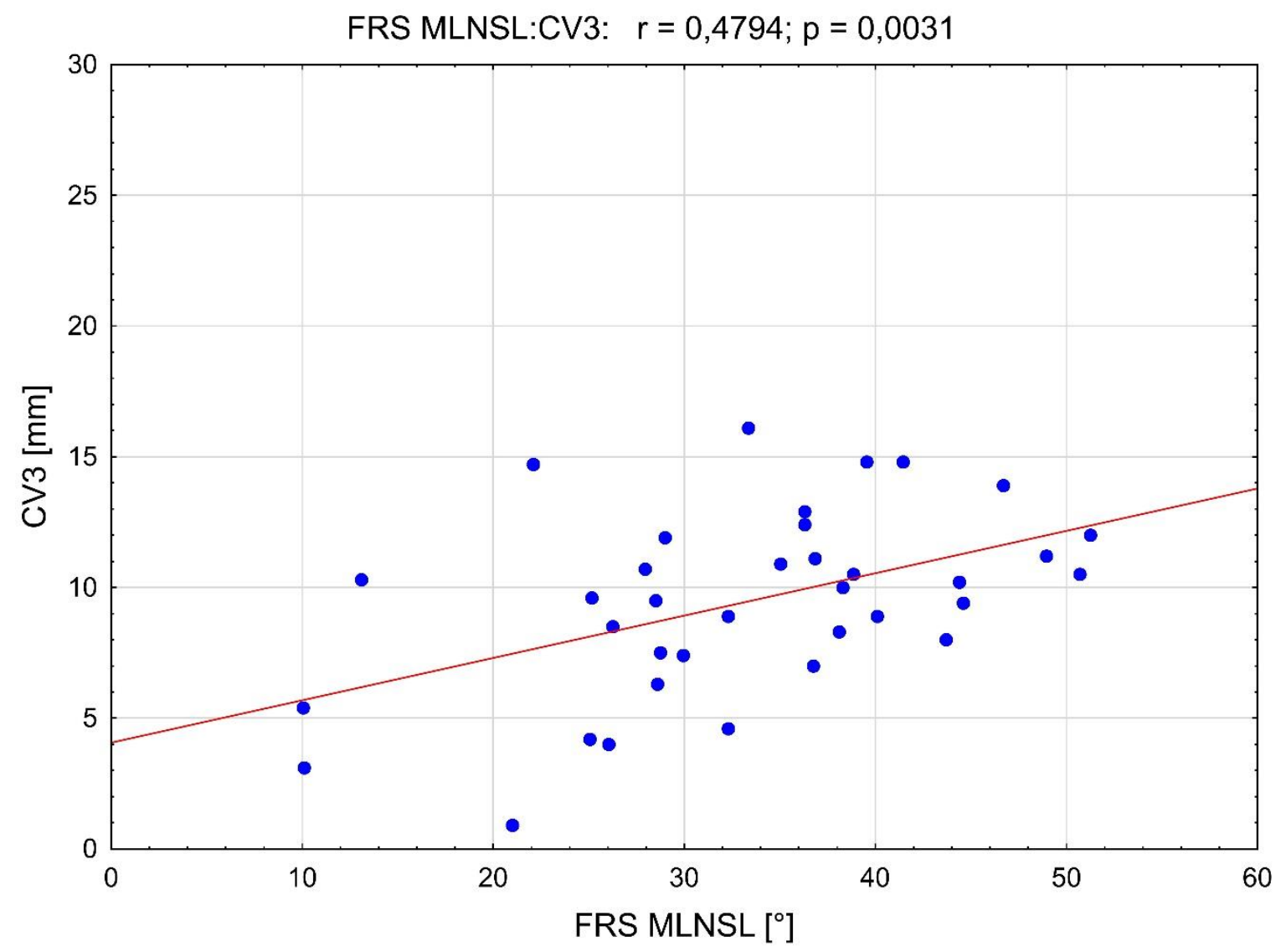

Abbildung 50

Scatterplot der Pearson-Korrelation zwischen dem Winkel ML-NSL $\left[{ }^{\circ}\right]$ und der Knochenhöhe des Kinns CV3 $[\mathrm{mm}]$

Zwischen dem Winkel ML-NSL und der vertikalen Dimension des knöchernen Kinns (CV3) besteht eine statistisch signifikante, positive Korrelation. Ein größerer ML-NSL korreliert mit einer vergrößerten Distanz zwischen der Wurzelspitze der Unterkiefer Front- bzw. Eckzähne und der Unterkieferbasis.

\begin{tabular}{|c|c|c|c|}
\hline \multicolumn{4}{|c|}{ Korrelationen zwischen OPG-Messungen und DVT-Messungen im Kinn } \\
\hline OPG Messung & DVT Messung (Kinn) & Korrelationskoeffizient & p-Wert \\
\hline WS1 & CV1 & 0,75 & 0,000 \\
\hline & CV2 & 0,83 & 0,000 \\
\hline & CV3 & 0,70 & 0,000 \\
\hline & CS4 & $-0,48$ & 0,003 \\
\hline & Vchin & 0,67 & 0,000 \\
\hline
\end{tabular}


Die besonders stark ausgeprägte Korrelation zwischen WS1 und CV2 ist zu erwarten, da es sich um analoge Messungen in zwei verschiedenen bildgebenden Verfahren handelt. Bemerkenswerter ist die negative, statistisch signifikante Korrelation zwischen WS1 (OPG) und CS4 (DVT).

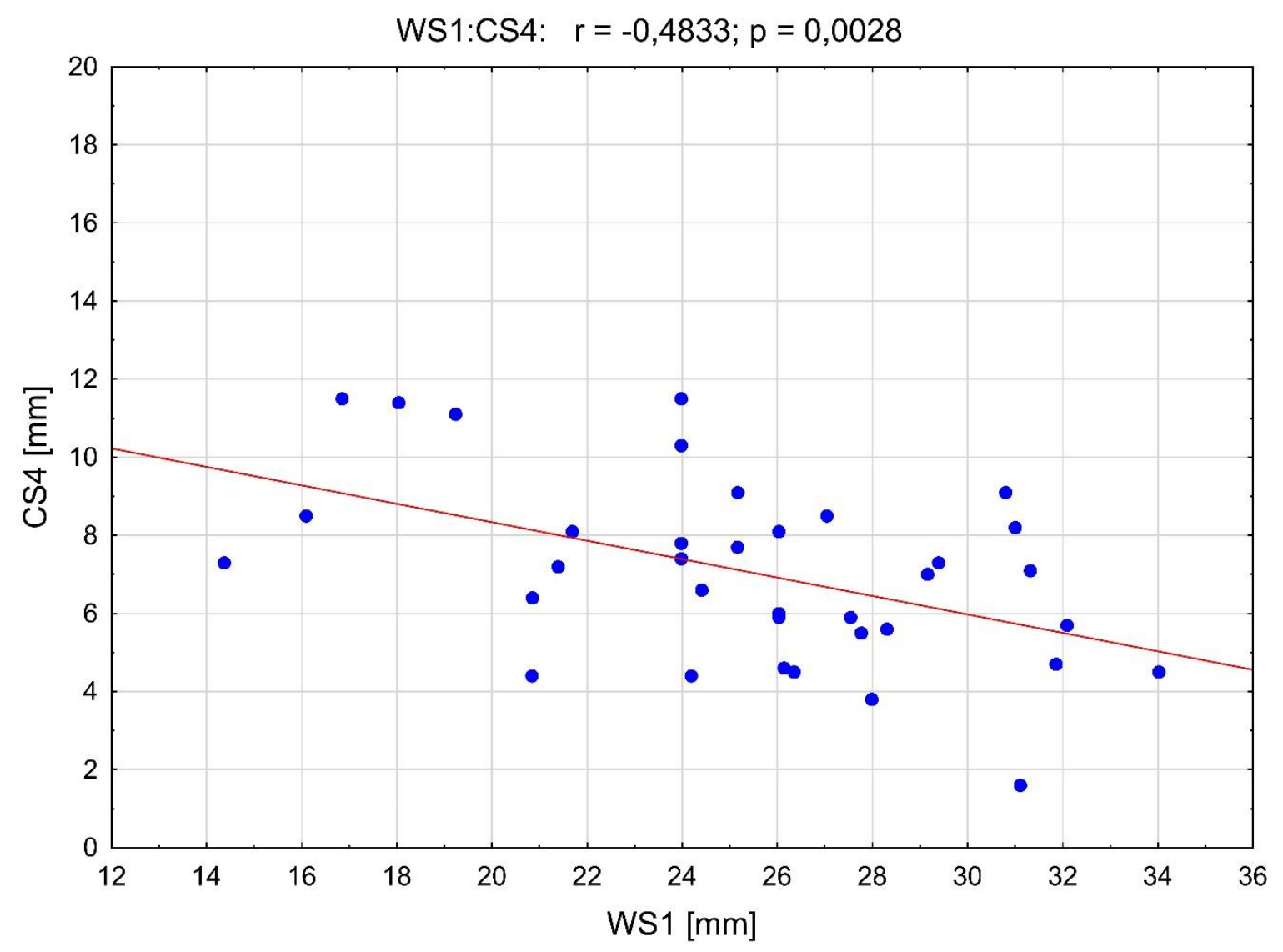

Abbildung 51

Scatterplot der Pearson-Korrelation zwischen den Strecken WS1 [mm] (gemessen im OPG) und CS4 [mm] (gemessen im DVT)

Anhand des Scatterplots wird erkenntlich, dass eine vergrößerte Distanz zwischen der Wurzelspitze der mittleren Unterkiefer Incisivi und der Unterkieferbasis im OPG (WS1) mit einem sagittal reduzierten Knochenangebot des Kinns (CS4) korreliert. Im OPG lässt sich die sagittale Dimension des Kinns nicht untersuchen.

Eine positive, statistisch signifikante Korrelation besteht außerdem zwischen WS1 und dem gesamten Knochenvolumen des Kinns. Auch hier erfolgt eine grafische Darstellung als Scatterplot. 


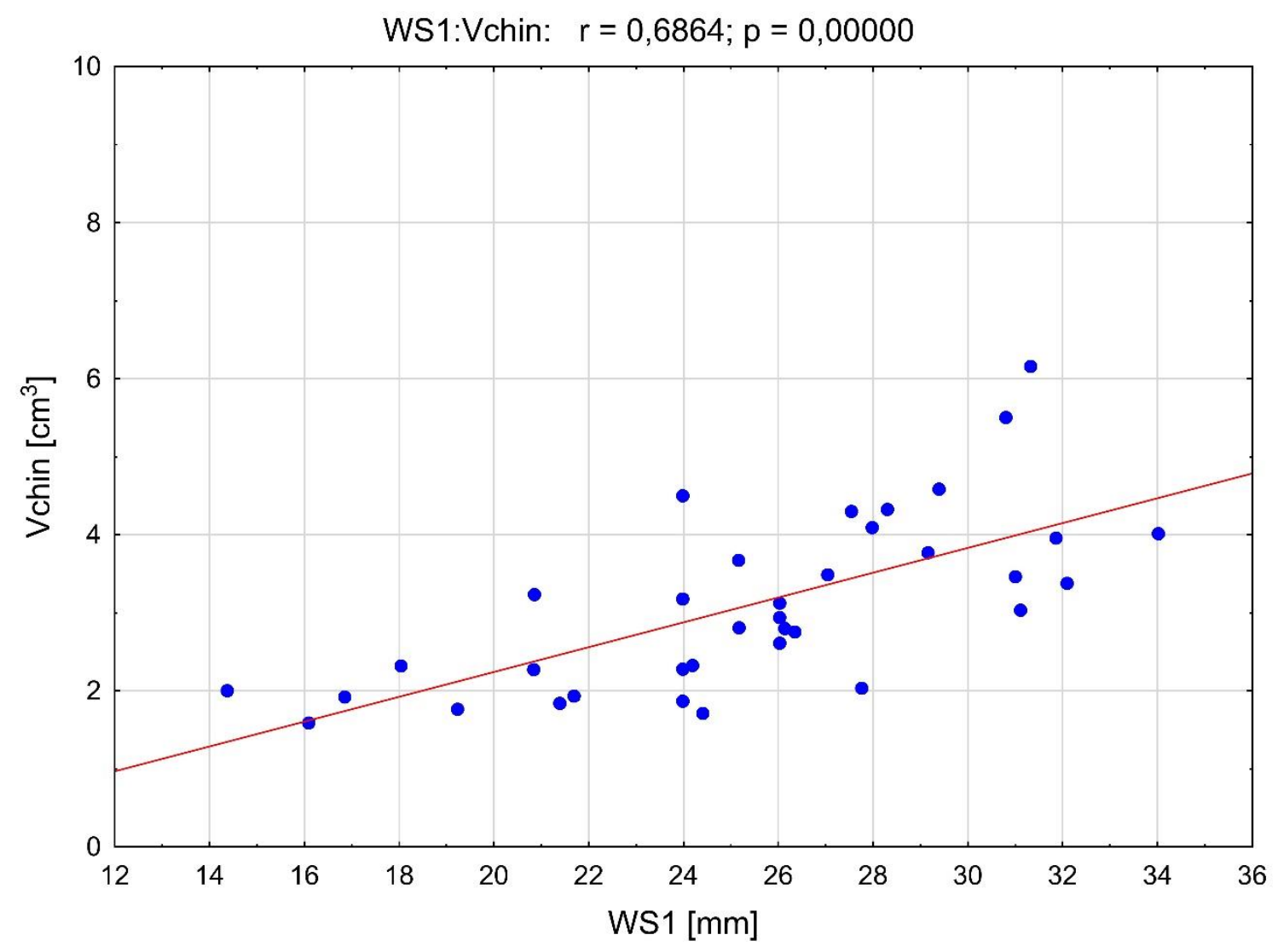

Abbildung 52

Scatterplot der Pearson-Korrelation zwischen der Strecke WS1 [mm] (im OPG gemessen) und dem Knochenvolumen des Kinns Vchin $\left[\mathrm{cm}^{3}\right]$

Dem Scatterplot lässt sich entnehmen, dass die Distanz zwischen der Wurzelspitze der mittleren Unterkiefer Incisivi und der Unterkieferbasis im OPG, positiv mit dem Knochenvolumen des Kinns korreliert.

3.3.4.2 Korrelationen mit DVT-Messwerten der retromolaren Region

\begin{tabular}{|c|c|c|c|}
\hline \multicolumn{3}{|c|}{ Korrelationen zwischen OPG-Messungen und retromolaren DVT-Messungen } \\
\hline OPG Messung & DVT Messung (retromolar) & Korrelationskoeffizient & p-Wert \\
\hline WS7 & MN1 & 0,51 & 0,002 \\
\hline & MN2 & 0,54 & 0,001 \\
\hline & MN3 & 0,41 & 0,013 \\
\hline & Vretro & 0,40 & 0,017 \\
\hline & MN3 & $-0,41$ & 0,013 \\
\hline
\end{tabular}


Es besteht eine negative Korrelation zwischen dem Kieferwinkel im OPG und dem vertikalen Knochenangebot oberhalb des Nervus alveolaris inferior. So korreliert ein vergrößerter Kieferwinkel mit einer reduzierten Distanz zwischen okklusaler Knochenoberfläche und Nerv.

Die im OPG gemessene Distanz zwischen den Wurzelspitzen des zweiten Unterkiefermolaren und der Unterkieferbasis korreliert positiv mit dem in der DVT-Aufnahme gemessenen vertikalen Knochenhöhe oberhalb des Nervus alveolaris inferior und dem dortigen Knochenvolumen. Für diese beiden statistisch signifikanten Korrelationen wurden Scatterplots erstellt.

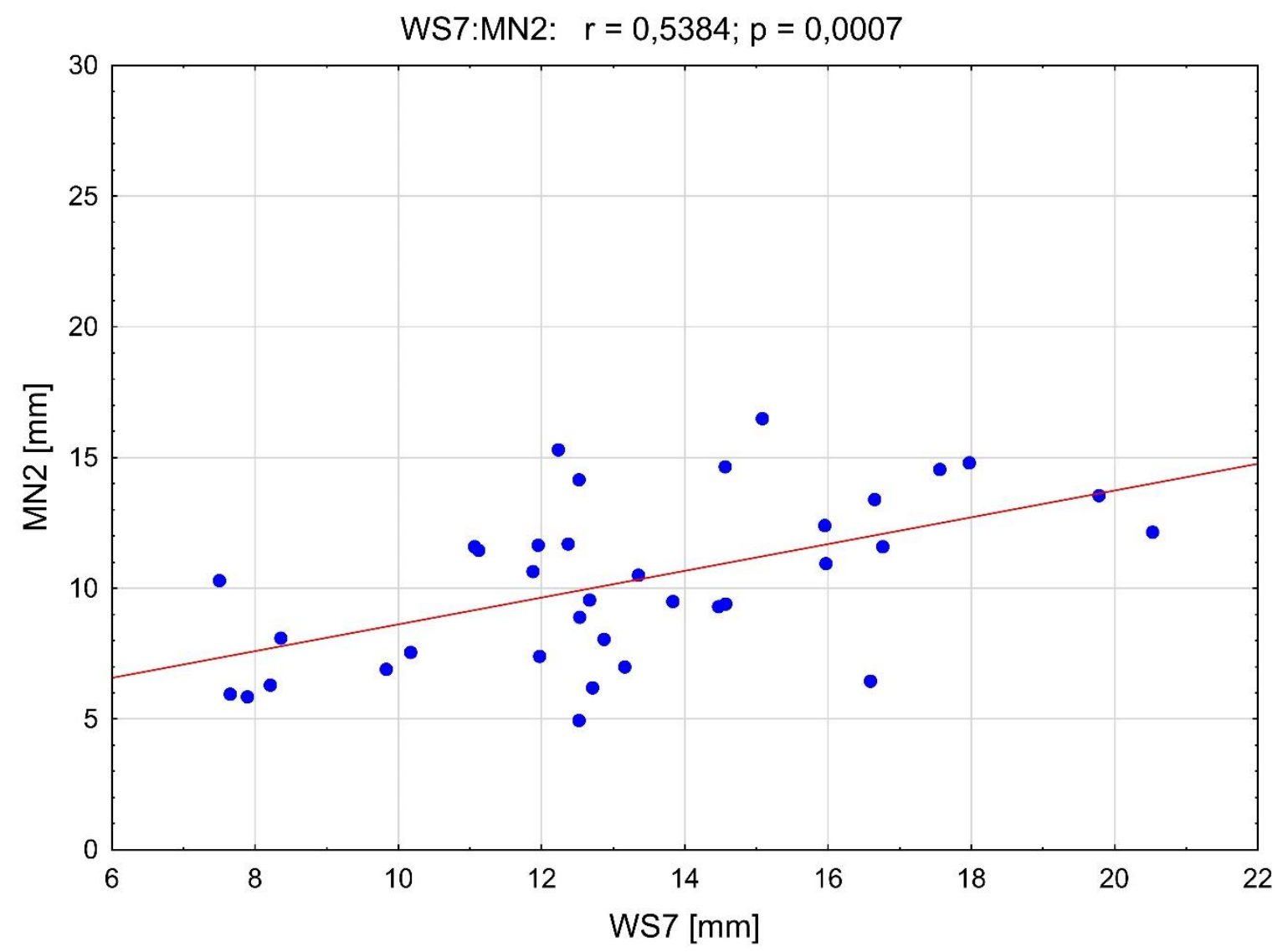




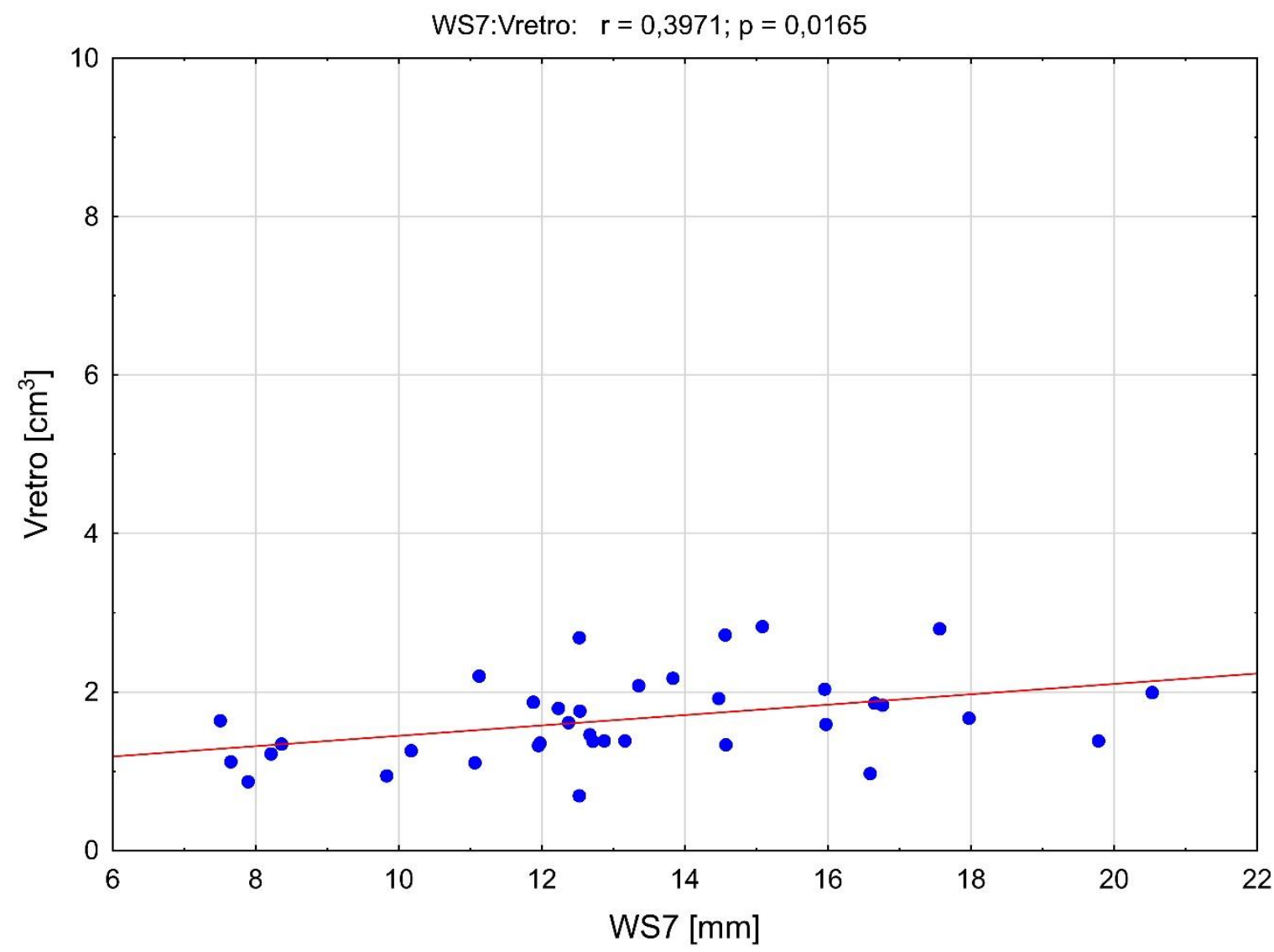

Abbildung $54 \quad$ Scatterplot der Pearson-Korrelation zwischen der Strecke WS7 [mm] (gemessen im OPG) und dem retromolaren Knochenvolumen Vretro $\left[\mathrm{cm}^{3}\right]$

Pearson-Korrelationen zwischen FRS-Messungen und DVT-Messungen der retromolaren Region, sowie zwischen FRS-Messungen und OPG-Messungen

\begin{tabular}{|c|c|c|c|}
\hline \multicolumn{4}{|c|}{ Korrelationen zwischen FRS-Messungen und retromolaren DVT-Messungen } \\
\hline FRS Messung & DVT Messung (retromolar) & Korrelationskoeffizient & p-Wert \\
\hline SNB & MN1 & $-0,34$ & 0,040 \\
\hline & MN2 & $-0,45$ & 0,006 \\
\hline ANB & MN3 & $-0,41$ & 0,012 \\
\hline & MN1 & 0,49 & 0,003 \\
\hline & MN2 & 0,57 & 0,001 \\
\hline Gn-Go-Ar & MN3 & 0,044 \\
\hline Hasund & Vretro & 0,34 & 0,039 \\
\hline Korrelationen zwischen & MN3 & 0,35 & 0,004 \\
\hline FRS Messung & MN3 & $-0,47$ & 0,002 \\
\hline Gn-Go-Ar & OPG Messung & Korrelationskoeffizient & p-Wert \\
\hline \multicolumn{2}{|c|}{ Ang. Mand. } & 0,89 & 0,000 \\
\hline
\end{tabular}


Der im FRS gemessene Kieferwinkel Gn-Go-Ar verhält sich bezüglich seiner negativen Korrelation zu dem vertikalen retromolaren Knochenangebot analog zu dem Kieferwinkel, der im OPG gemessen wurde. Die äußerst starke positive Korrelation zwischen Gn-Go-Ar und Ang. Mand. war zu erwarten, da es sich um analoge Messungen in zwei verschiedenen bildgebenden Verfahren handelt.

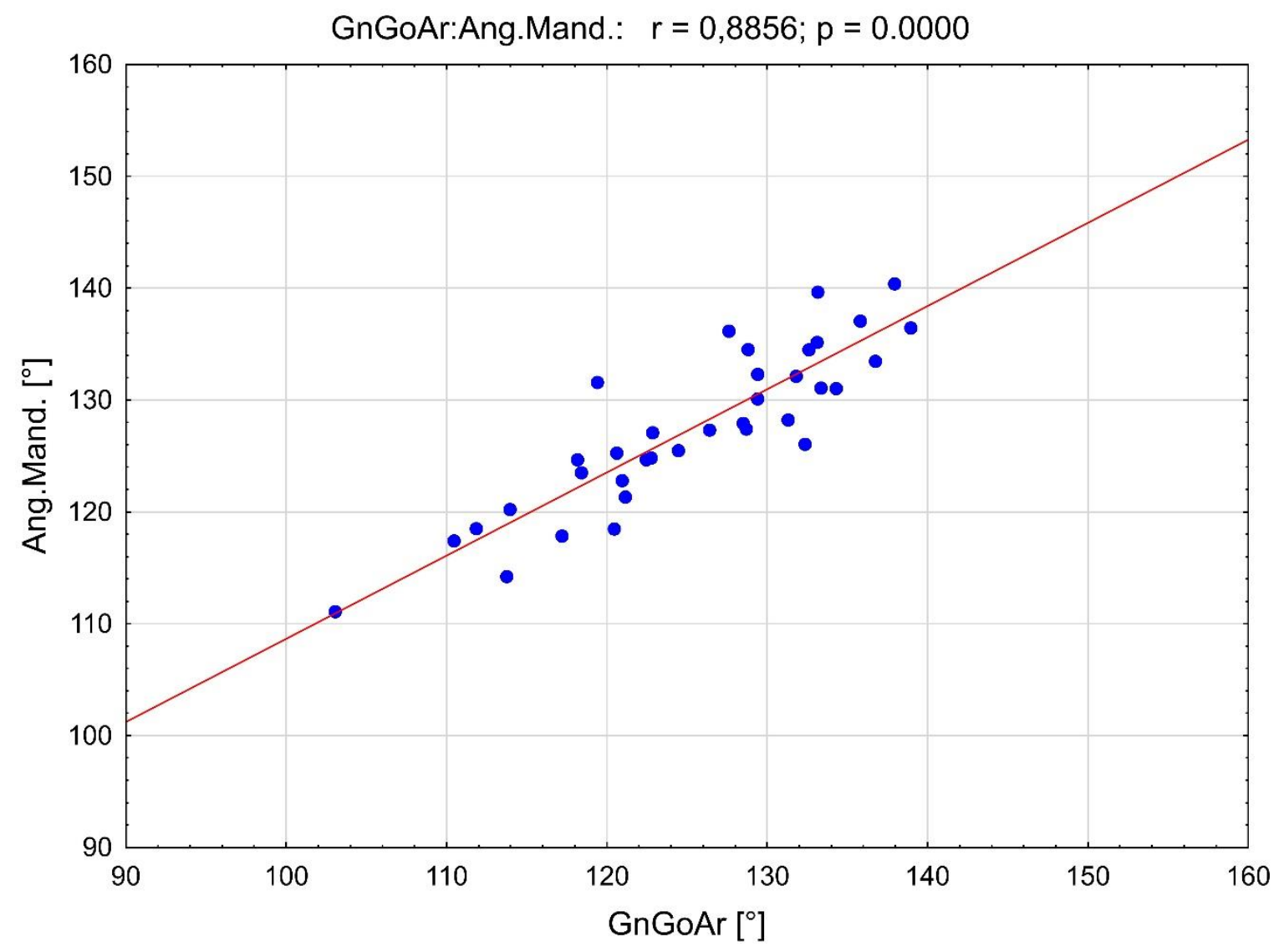

Abbildung 55 Scatterplot der Pearson-Korrelation zwischen dem Gn-Go-Ar [ [ ${ }^{\circ}$ (gemessen im FRS) und dem Ang. Mand. $\left[^{\circ}\right]$ (gemessen im OPG)

Basal sagittal distale Kieferrelationen, gekennzeichnet durch einen erhöhten ANB im FRS, korrelieren positiv mit dem vertikalen Knochenangebot (MN) und gesamten retromolaren Knochenvolumen (Vretro). Auch für diese statistisch signifikante Korrelation wurde ein Scatterplot erstellt. 


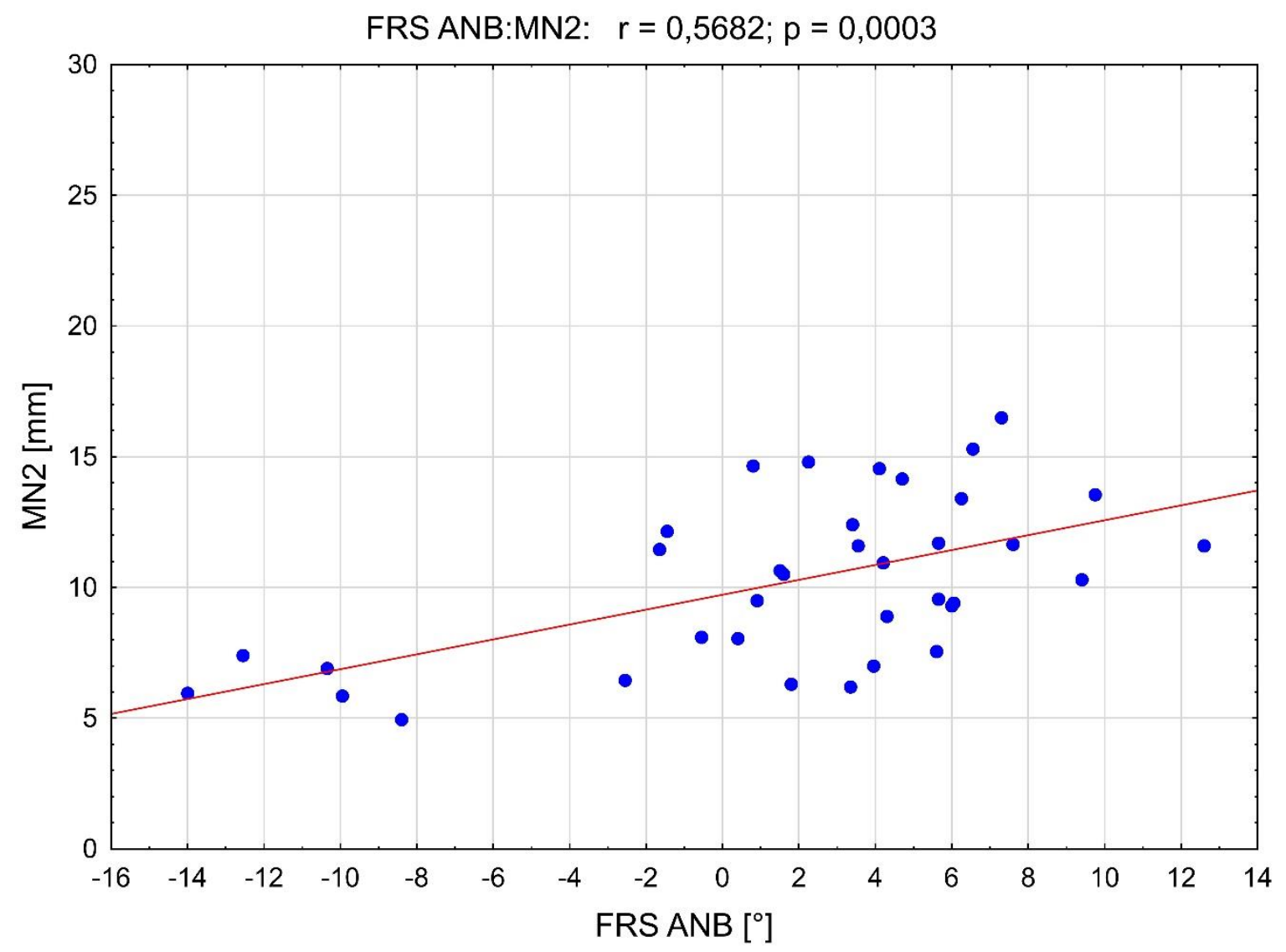

Abbildung 56

Scatterplot der Pearson-Korrelation zwischen dem ANB $\left[^{\circ}\right]$ (gemessen im FRS) und der Höhe des retromolaren Knochenblockes MN2 [mm] (gemessen im DVT)

Ähnlich wie im Kinn lassen sich auch in der retromolaren Knochenregion bestimmte kephalometrische Wachstumsformen und -typen mit Messungen in DVT-Aufnahmen in Verbindung bringen. Basal sagittal distale Relationen (ANB vergrößert), Retrognathien (SNB verkleinert) und anteriore Rotationen (Hasund-Score vergrößert und Gn-Go-Ar verkleinert) korrelieren in der retromolaren Region mit einem vermehrten Knochenangebot. Ein vergrößerter Kieferwinkel hingegen korreliert mit verringertem Knochenangebot in der retromolaren Region.

\subsubsection{Korrelationen mit DVT-Messwerten der Crista zygomatico-alveolaris}

Die statistisch signifikanten Korrelationen im Bereich der Crista zygomatico-alveolaris beschränkten sich auf jene mit der retromolaren Region. Diese waren durchweg positiv, sodass eine Crista mit stärkerer Knochenwand und größerem Volumen mit einem erhöhten retromolaren Knochenangebot korreliert. 
Tabelle 21 Pearson-Korrelationen zwischen DVT-Messungen der Crista zygomatico-alveolaris und DVTMessungen der retromolaren Region

\section{Korrelationen mit DVT-Messungen der Crista zygomatico-alveolaris}

\begin{tabular}{|c|c|c|c|}
\hline DVT Messung (Crista) & DVT Messung (retromolar) & Korrelationskoeffizient & p-Wert \\
\hline ZA8 & MN1 & 0,51 & 0,002 \\
\hline & MN2 & 0,45 & 0,007 \\
\hline & MW2 & 0,44 & 0,008 \\
\hline & MW3 & 0,35 & 0,039 \\
\hline & MW5 & 0,55 & 0,001 \\
\hline & MW9 & 0,35 & 0,039 \\
\hline & MN1 & 0,48 & 0,003 \\
\hline & MN2 & 0,45 & 0,002 \\
\hline
\end{tabular}

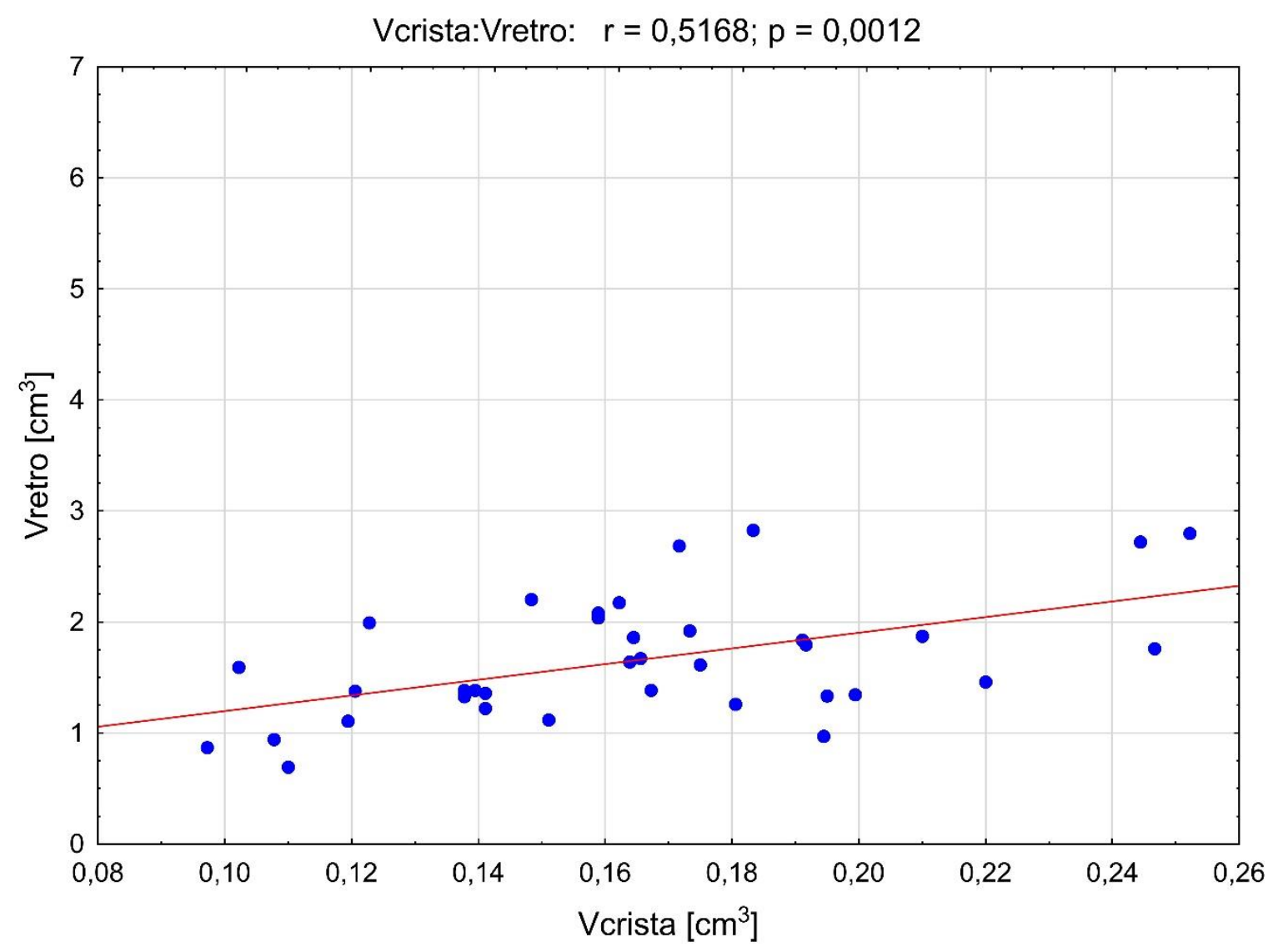




\section{Diskussion}

Im Rahmen dieser Arbeit wurde die Aussagekraft der geläufigsten und wichtigsten Röntgenverfahren der Zahn- Mund- und Kieferheilkunde verglichen. Dieser Vergleich dient der Darstellung von Differenzen, Korrelationen und dem diagnostischen Mehrwert der jeweiligen Methode bei der Suche nach geeigneten intraoralen Knochenspenderregionen für die Gewinnung von Transplantaten zum Alveolarkammaufbau. Statistisch signifikante Korrelationen zwischen kephalometrischen Wachstumsmustern und den Knochendimensionen intraoraler Entnahmestellen für die Knochentransplantation konnten nachgewiesen werden. Diese könnten bei der gezielten Suche nach geeigneten Spenderregionen und der Indikationsstellung, sowie Rechtfertigung für weitere diagnostische Hilfsmittel wie die DVT, hilfreich sein.

\subsection{Diskussion von Material und Methode}

In der vorliegenden Arbeit wurden Messungen an Aufnahmen von drei unterschiedlichen zahnmedizinischen Röntgenverfahren durchgeführt. Die hierbei angewandten Methoden kamen in zahlreichen Studien bereits zur Anwendung und sind fester Bestandteil der alltäglichen Routinediagnostik zahnmedizinischer Fachgebiete.

Die Fernröntgenseitenanalyse ist ein wichtiger Bestandteil der kieferorthopädischen Diagnostik (Hofrath 1931). Die ursprünglich manuelle Analyse konventioneller Röntgenaufnahmen wurde inzwischen durch die softwaregestützte Auswertung digitaler Aufnahmen, welche in dieser Arbeit zur Anwendung kam, weitestgehend verdrängt. Diese scheint keine signifikanten Ungenauigkeiten gegenüber der manuellen Methode aufzuweisen und zeichnet sich zudem durch eine wesentliche Zeitersparnis aus (Santoro et al. 2006; Sayinsu et al. 2007; Celik et al. 2009). Diese Analyse ermöglicht anhand fest definierter, reproduzierbarer Referenzpunkte eine kieferorthopädische Diagnose, Therapieplanung und Verlaufskontrolle des Schädelwachstums. Auf diese Weise wird eine FRS-Aufnahme auch für die kieferorthopädische Therapie von Patienten mit Nichtanlagen untersucht. Häufig steht bei diesen Patienten eine implantologische Versorgung nach kieferorthopädischer Therapie an. 
Die im Rahmen dieser Arbeit gewonnen Erkenntnisse könnten den Stellenwert der FRSAufnahme bei der implantologischen Versorgung dieser Patienten steigern.

Das zweite hier untersuchte Röntgenverfahren ist das OPG. Die erstellten Panoramaschichtaufnahmen können dem Untersucher eine generelle Übersicht des Viszerokraniums verschaffen und werden seltener als FRS-Aufnahmen für standardisierte Messungen und Analysen herangezogen. Vorausgehende Studien untersuchten die Genauigkeit von Messungen an OPG-Aufnahmen, wie sie in dieser Arbeit erfolgten. Lineare Messungen und das Bestimmen von Winkeln in OPG-Aufnahmen wurden mit direkten Messungen am Kieferknochen von Körperspendern verglichen. Hierbei zeigte sich keine statistisch signifikante Differenz der erhobenen Messwerte (Provedel et al. 2016). Andere Studien führten Vergleiche von am FRS und OPG erhobenen Messwerten durch. Hierbei konnten keine statistisch signifikanten Unterschiede zwischen den Messwerten des Kieferwinkels aus FRS- und OPG-Aufnahmen festgestellt werden. (Okşayan et al. 2012; Zangouei-Booshehri et al. 2012). Es ist jedoch zu beachten, dass die Genauigkeit des messbaren Kieferwinkels in OPGAufnahmen von der Neigung des Kopfes in der Sagittalebene abhängig sein kann. Eine solche Neigung hätte auf den Messwert des Kieferwinkels in FRS-Aufnahmen keinen Einfluss. Daher sollte der Kopf des Patienten im OPG entsprechend der Frankfurter Horizontale positioniert werden.

Das dritte und letzte untersuchte Röntgenverfahren ist die DVT. Messungen von Knochenvolumina möglicher Spenderregionen in CT- und DVT-Aufnahmen mit geeigneten Analysesoftwares erfolgten bereits in zahlreichen Studien (Cavalcanti und Vannier 1998; Fuentes et al. 2015; Zeltner et al. 2016). Weitere Arbeiten untersuchten die Genauigkeit dieser Messmethoden, indem die Schädel menschlicher Leichen sowohl manuell, als auch röntgenologisch untersucht wurden. Im Vergleich zu direkten Messungen an Leichen erwiesen sich die Messungen am Röntgenabbild als zuverlässig (Cavalcanti et al. 2004; Liu et al. 2006; Berco et al. 2009). Bei der Knochenblockentnahme an diesen Leichen wurden, entsprechend dem klinischen Vorgehen, Sicherheitsabstände zu sensiblen anatomischen Strukturen wie der lingualen Kortikalis, den Zahnwurzeln und Nerven oder Gefäßen eingehalten. Die angewandten Sicherheitsabstände im Rahmen dieser Arbeit kamen bereits in mehreren Studien zur Anwendung (Hoppenreijs et al. 1992; Yavuz et al. 2009; Zeltner et al. 2016). 


\subsubsection{Diskussion der statistischen Methoden}

Um abschließend die Reproduzierbarkeit der angewandten Messmethoden zu prüfen, wendet ein zweiter Rater diese an den gleichen Aufnahmen an. Für die Knochenvolumenberechnungen wurde so die Inter-Rater Reliabilität bestimmt. Hierfür stehen prinzipiell verschiedene statistische Methoden zur Verfügung. Mit Hilfe der Intraclass correlation erhält der Untersucher einen Wert zwischen 0 und 1. Während bei einem Wert von 1 eine vollständige Übereinstimmung sämtlicher Messungen beider Rater vorläge, gäbe es bei einem Wert von 0 keine Übereinstimmungen. Allerdings lässt sich anhand dieses Wertes keine Aussage zu der klinischen Relevanz treffen, da das Ergebnis nicht in der ursprünglichen Einheit der Rohdaten vorliegt. Der in dieser Arbeit genutzte Bland-Altman-Plot hingegen versorgt den Anwender mit Informationen in der Maßeinheit der erhobenen Rohdaten. Hiermit lässt sich eine Aussage zu der klinischen Relevanz treffen und ein Toleranzbereich der Messungen zwischen oberem und unterem Limit of Agreement ermitteln (Bland und Altman 1986). Mit Hilfe der Bland-Altman-Methode gewinnt der Anwender Erkenntnisse über die Intervalle in denen sich $95 \%$ der Messdifferenzen befinden. Diese sind in der Tabelle 9 dargestellt. Betrachtet man diese Intervalle für die drei berechneten Knochenvolumina, so fällt eine Beziehung zwischen der Größe des durchschnittlichen Knochenvolumens und der Größe des Intervalls für die Messdifferenzen auf. Die durchschnittlichen Knochenvolumina und Messdifferenzen sind in aufsteigender Reihenfolge Vcrista, Vretro und Vchin. Eine mögliche Erklärung ist der angewandte Vergrößerungsfaktor bei der Vermessung dieser Strecken. Während bei der Vermessung der Crista zygomatico-alveolaris ein deutlich intensiverer Gebrauch der Vergrößerungsfunktion gemacht wird, arbeitet der Untersucher im Bereich des Kinns kaum mit der implementierten Vergrößerungsfunktion der Software. Messungenauigkeiten werden dadurch amplifiziert bzw. reduziert. So ergibt sich für das Knochenvolumen der Crista ein Differenzintervall von $0,0149 \mathrm{~cm}^{3}$, während für das Knochenvolumen des Kinns ein Differenzintervall von $0,173 \mathrm{~cm}^{3}$ vorliegt. Ein weiterer möglicher Einfluss könnten die festgelegten Dimensionen der Transplantate sein. Während im Bereich der Crista zygomatico-alveolaris die Höhe und Breite des Transplantates mit $10 \mathrm{~mm}$ bereits festgelegt ist, sind diese Dimensionen im Kinn variabel. Hierdurch könnte im Kinn ein größeres Potential für Messdifferenzen entstanden sein. In Anbetracht der vorliegenden Daten dürfte die Inter-Rater Reliabilität dennoch als suffizient erachtet werden. 
Der Bland-Altman Plot wurde auch bei den zweidimensionalen Röntgenaufnahmen angewandt. Auffällig ist, dass bei der Messung des Kieferwinkels im FRS (GnGoAr) ein größeres Limit of Agreement entstand, als bei der Messung des Kieferwinkels im OPG (Ang. Mand.). Im FRS entstand dadurch ein Intervall von 4,1 ${ }^{\circ}$, während im OPG ein kleineres Differenzintervall von $3,73^{\circ}$ zustande kam. Die Abwesenheit von Doppelkonturen des Kieferwinkels im OPG könnte zu diesem Ergebnis beigetragen haben. Ob dieser geringfügige Unterschied der Inter-Rater Reliabilität schlussendlich praxisrelevant ist, kann in Frage gestellt werden. 
4.2 Diskussion der Ergebnisse

Die durchschnittlichen Messwerte des untersuchten Patientenkollektivs entsprechen alle den Sollwerten nach Hasund (Segner und Hasund 1998).

Tabelle 22 Durchschnittlich gemessene FRS Werte und deren Sollwerte nach Hasund. Angabe der FRS-Winkel erfolgt in Grad [ ${ }^{\circ}$ ], Index in Prozent [\%], Hasund als Punktwert.

\begin{tabular}{|c|c|c|}
\hline Parameter & Mittelwert im Patientenkollektiv [ ${ }^{\circ}$ ] & Sollwert nach Hasund $\left.\mathbf{~}^{\circ}\right]^{\circ}$ \\
\hline SNA & 80,16 & $82,0 \pm 3$ \\
\hline SNB & 78,16 & $80,0 \pm 3$ \\
\hline ANB & 1,88 & $2,0 \pm 2$ \\
\hline ML-NSL & 33,26 & $32,0 \pm 6$ \\
\hline NL-NSL & 9,11 & $8,5 \pm 3$ \\
\hline ML-NL & 24,15 & $23,5 \pm 3$ \\
\hline NSBa & 131,89 & $130,0 \pm 6$ \\
\hline Gn-Go-Ar & 125,33 & $126,0 \pm 10$ \\
\hline Index [\%] & 77,36 & $79,0 \pm 5$ \\
\hline Hasund [Punkte] & 2,47 & 3 \\
\hline
\end{tabular}

Bei der Betrachtung einzelner Patienten sind jedoch zahlreiche Parameter ober- beziehungsweise unterhalb der genannten Sollwerte.

Tabelle $23 \quad$ Totale und relative (\%) Sollwert-Abweichungen der FRS-Messwerte im untersuchten Patientenkollektiv

\begin{tabular}{|c|c|c|c|}
\hline Parameter & $\mathbf{n}$ Über Sollwert & $\mathbf{n}$ Unter Sollwert & $\mathbf{n}$ Sollwert entsprechend \\
\hline SNA & $5(14 \%)$ & $14(39 \%)$ & $17(47 \%)$ \\
\hline SNB & $10(28 \%)$ & $16(44 \%)$ & $10(28 \%)$ \\
\hline ANB & $16(44 \%)$ & $9(25 \%)$ & $11(31 \%)$ \\
\hline ML-NSL & $13(36 \%)$ & $7(19 \%)$ & $18(50 \%)$ \\
\hline NL-NSL & $10(28 \%)$ & $8(22 \%)$ & $9(25 \%)$ \\
\hline ML-NL & $16(44 \%)$ & $11(31 \%)$ & $24(67 \%)$ \\
\hline NSBa & $9(25 \%)$ & $3(8 \%)$ & $30(83 \%)$ \\
\hline Gn-Go-Ar & $1(3 \%)$ & $5(14 \%)$ & $19(53 \%)$ \\
\hline Index & $4(11 \%)$ & $13(36 \%)$ & $30(83 \%)$ \\
\hline Hasund & $4(11 \%)$ & $2(6 \%)$ & $16)$ \\
\hline
\end{tabular}


Die zahlreichen Normabweichungen innerhalb des untersuchten Patientenkollektivs waren zu erwarten, da es sich bei einem großen Anteil der FRS-Aufnahmen um präoperative Untersuchungen bei Umstellungsosteotomien handelt. Einige dieser ausgeprägten Kieferfehlstellungen können nur mit einer zusätzlichen kieferchirurgischen Intervention behoben werden (Cunningham und Johal 2015). Zugleich ermöglichen diese Normabweichungen überhaupt erst eine Untersuchung hinsichtlich der Korrelationen zwischen kephalometrischen Parametern und dem Knochenvolumen, da eine Varianz der Messwerte Voraussetzung hierfür ist. Eine radiologische Untersuchung bei gesunden Patienten wäre zudem aus ethischen Gründen heute undenkbar.

\subsubsection{Korrelationen zwischen FRS-Messwerten und Knochendimensionen im DVT}

\subsubsection{Korrelationen zwischen FRS-Messerwerten und Knochendimensionen des Kinns}

Die im FRS gemessenen Winkel ML-NSL und ML-NL weisen mit allen drei Dimensionen des knöchernen Kinns statistisch signifikante Korrelationen auf. Diese Korrelation ist positiv zwischen der Länge (CV) und negativ zwischen sowohl der Breite $(\mathrm{CH})$, als auch Dicke (CS) des knöchernen Kinns. Ein zunehmender ML-NSL korreliert folglich mit einem dünneren und schmaleren, aber vertikal längeren Kinn. Bei Patienten mit vertikalem Wachstumsmuster könnte daher der interforaminale Abstand und die Distanz zur lingualen Kortikalis eher ein limitierender Faktor bei der Knochenentnahme sein, als der Abstand zwischen Unterkieferbasis und den Apices der Incisivi. Eine solche Verschmälerung des Alveolarfortsatzes bei vertikalem Wachstumstyp wurde 1996 von Handelman beschrieben und stellt zusammen mit der Elongation der mandibulären Incisivi einen kompensatorischen Mechanismus dar (Handelman 1996; Kuitert et al. 2006). Da hierbei die Distanz zwischen den Apices der Incisivi und der Unterkieferbasis vergrößert wird, könnte in solchen Fällen möglicherweise mehr Knochen in der vertikalen Dimension (CV) entnommen werden. Diese Beteiligung der knöchernen Dimensionen des Kinns an solchen kompensatorischen Mechanismen konnte in vorangehenden Arbeiten nachgewiesen werden (Beckmann et al. 1998b; Beckmann et al. 1998a; Sadek et al. 2015).

Der ML-NL verhält sich diesbezüglich analog zum ML-NSL. Auch hier besteht eine positive Korrelation zu der Länge (CV) und negative Korrelationen zu der Breite (CH) und Dicke (CS). 
Allerdings sind diese Korrelationen geringfügig schwächer ausgeprägt als bei dem ML-NSL (Tabelle 17). Ursache hierfür könnte die zusätzliche maxilläre Komponente des ML-NL sein, die bei dem ML-NSL nicht vorhanden ist. So wäre auch bei einer anterioren Rotation der Maxilla eine Vergrößerung des ML-NL die Folge. Dies hat jedoch keinen unmittelbaren Einfluss auf die Rotation und sagittale Lage des Unterkiefers, die als Erklärung für die knöchernen Dimensionen des Kinns Bereichs nutzbar wären.

Die Korrelationen einer posterioren Rotation der Mandibula mit den Dimensionen des Kinns ließen sich auch im Hasund-Score nachweisen. Ein geringer Hasund-Score beschreibt (wie auch ein großer ML-NSL) eine posteriore Rotation der Mandibula und korreliert ebenfalls positiv mit der Länge (CV) und negativ mit der Breite $(\mathrm{CH})$ und Dicke $(\mathrm{CS})$ des Kinns.

Trotz der Tatsache, dass zahlreiche FRS-Variablen mit allen drei gemessenen Dimensionen des Kinns zugleich korrelieren, bestehen kaum direkte und signifikante Korrelationen zu dem eigentlichen Knochenvolumen (Vchin). Da es sich bei dem Volumen um ein Produkt der drei Strecken ( $\mathrm{CV}, \mathrm{CH}, \mathrm{CS})$ handelt, können gegenläufige Veränderungen, wie eine zunehmende Höhe bei abnehmender Breite und Dicke, das Volumen weitestgehend unverändert lassen oder zumindest den Einfluss auf das Volumen reduzieren. Der einzige FRSParameter mit direkter Korrelation zu dem Knochenvolumen des Kinns im DVT ist der Index (Abb. 7). Durch das Dividieren der oberen Gesichtshöhe (Strecke N - Sp') durch die Untere (Strecke Sp'-Gn) und die anschließende Multiplikation mit 100, kann man den Index in Prozent errechnen. Die Korrelation zwischen Index und Vchin ist negativ, weil ein geringer Index, bedingt durch eine relativ vergrößerte untere Gesichtshöhe, mit hohem Knochenvolumen im Kinn korreliert. Dies war zu erwarten, da die untere Gesichtshöhe im Index als Divisor fungiert und zu einem maßgeblichen Anteil von der vertikalen Dimension des Kinns bestimmt wird. Die Winkel ML-NSL und ML-NL erfassen die vertikale Dimension des Kinns nur indirekt. 
Ein weiterer möglicher Grund weshalb der Index signifikant mit Vchin korreliert und die Winkel ML-NL und ML-NSL nicht, ist der Einfluss der Distanz zwischen vorderer Gesichtshöhe (Strecke N - Gn) und hinterer Gesichtshöhe (Strecke S - tGo) auf diese beiden Winkel. Ist lediglich die Distanz zwischen vorderer und hinterer Gesichtshöhe reduziert, so macht sich dies in den Winkeln ML-NL und ML-NSL bemerkbar. Der Index hingegen bliebe unverändert, wie möglicherweise auch die vertikale Dimension (CV) und das Knochenvolumen des Kinns (Abb. 58).
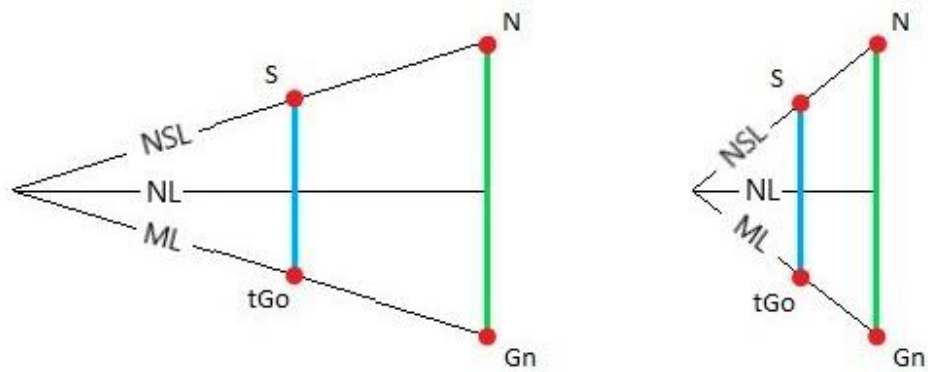

Abbildung 58

Schematische Darstellung des möglichen Einflusses der Distanz zwischen vorderer und hinterer Gesichtshöhe auf die Winkel ML-NL und ML-NSL. Sella (S), Nasion (N), Gnathion (Gn), Goniontangentenpunkt (tGo), hintere Gesichtshöhe (blau), vordere Gesichtshöhe (grün).

Bei Beobachtung der Korrelationskoeffizienten für den ML-NSL und die Knochendicke des Kinns (CS) fällt auf, dass der Koeffizient von der Unterkieferbasis in Richtung des Alveolarfortsatzes zunimmt (Abb. 59). Außerdem besteht ein deutlicher Unterschied zwischen den kranialen drei Messpunkten (CS1, CS4, CS7) bezüglich ihrer Korrelation mit dem MLNSL, wobei der mittige Messpunkt (CS4) die stärkste Korrelation aufweist.

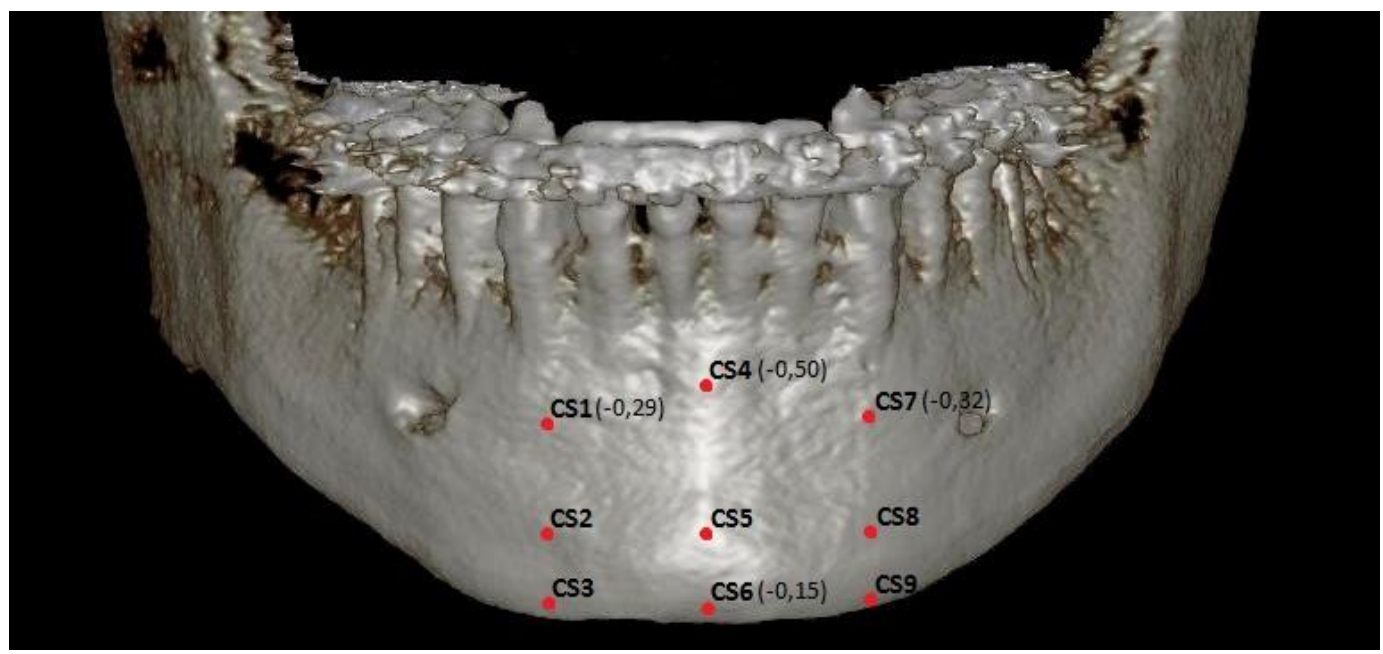
chenden Pearson Korrelationen zu dem ML-NSL (Klinik für Mund-, Kiefer- und Gesichtschirurgie Universitätsklinikum Göttingen) 
In Abbildung 59 ist zu erkennen, dass CS4 eine engere Lagebeziehung zum Alveolarfortsatz hat, als CS1 und CS7. Ursächlich hierfür ist der einzuhaltende Sicherheitsabstand zu den jeweiligen Wurzelspitzen von $5 \mathrm{~mm}$. Die längeren Wurzeln der Eckzähne bedingen daher die Lage der Messpunkte CS1 und CS7. Die Tatsache, dass mit zunehmender Nähe zum Alveolarfortsatz die negative Korrelation zwischen Knochendicke und ML-NSL zunimmt, könnte sich durch die charakteristischen Wachstumsmuster und -zonen der Mandibula erklären lassen. Mit Hilfe von implantierten Tantalstiften in ausgewählten, über das Wachstum hinweg lagestabilen Punkten der Maxilla und Mandibula, untersuchte Björk diese Wachstumsmuster (Bjork 1963). Hierbei dienten die röntgenopaken Implantate als Referenzpunkte bei der Überlagerung von zeitlich versetzten FRS Aufnahmen, die über einen Zeitraum von 6 Jahren erstellt wurden. Mit Hilfe dieser Technik konnten Richtung und Ausmaß des Wachstums beobachtet werden. Unter Anderem stellte sich heraus, dass im Bereich des Alveolarfortsatzes (Regio CS4, Abb. 59 und 60) eine deutlichere Zunahme von Knochen am Kinn stattfand, als an den in der Nähe der Basis gelegenen Anteilen (Regio CS6, Abb. 59 und 60). Ein vertikales Wachstumsmuster, das durch einen vergrößerten ML-NSL beschrieben wird, korreliert mit einem sagittal dünneren Kinn. Umgekehrt korreliert ein horizontales Wachstumsmuster, mit kleinerem ML-NSL, mit einem sagittal dickeren Kinn. Diese Korrelationen scheinen an den von Bjork beschriebenen Wachstumszentren der Kinnregion besonders ausgeprägt zu sein (Abb. 59 und 60). Interindividuelle Wachstumsunterschiede machen sich in diesen Wachstumszentren besonders bemerkbar. Diese könnten die höhere Korrelation und Standardabweichung für Messpunkte in Alveolarfortsatznähe erklären. Das Wachstum ist hierbei an der dorsalen Fläche der Symphyse ausgeprägter als ventral (Abb. 60) (Bjork 1963). Neuere Untersuchungen konnten diese Beobachtung mit Hilfe von computertomografischen Aufnahmen reproduzieren (Krarup et al. 2005).

\section{kranial}

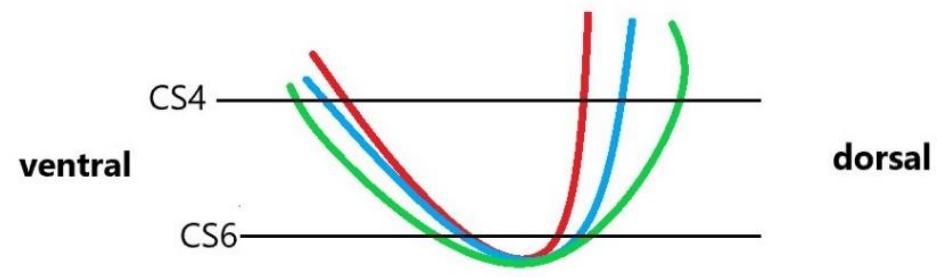

kaudal 
Die horizontale Breite des Kinns $(\mathrm{CH})$ weist ebenfalls eine negative Korrelation zum ML-NSL auf. Allerdings ist diese negative Korrelation mit zunehmender Nähe zu der Unterkieferbasis ansteigend, statt wie bei der Kinndicke abnehmend (Abb. 61).

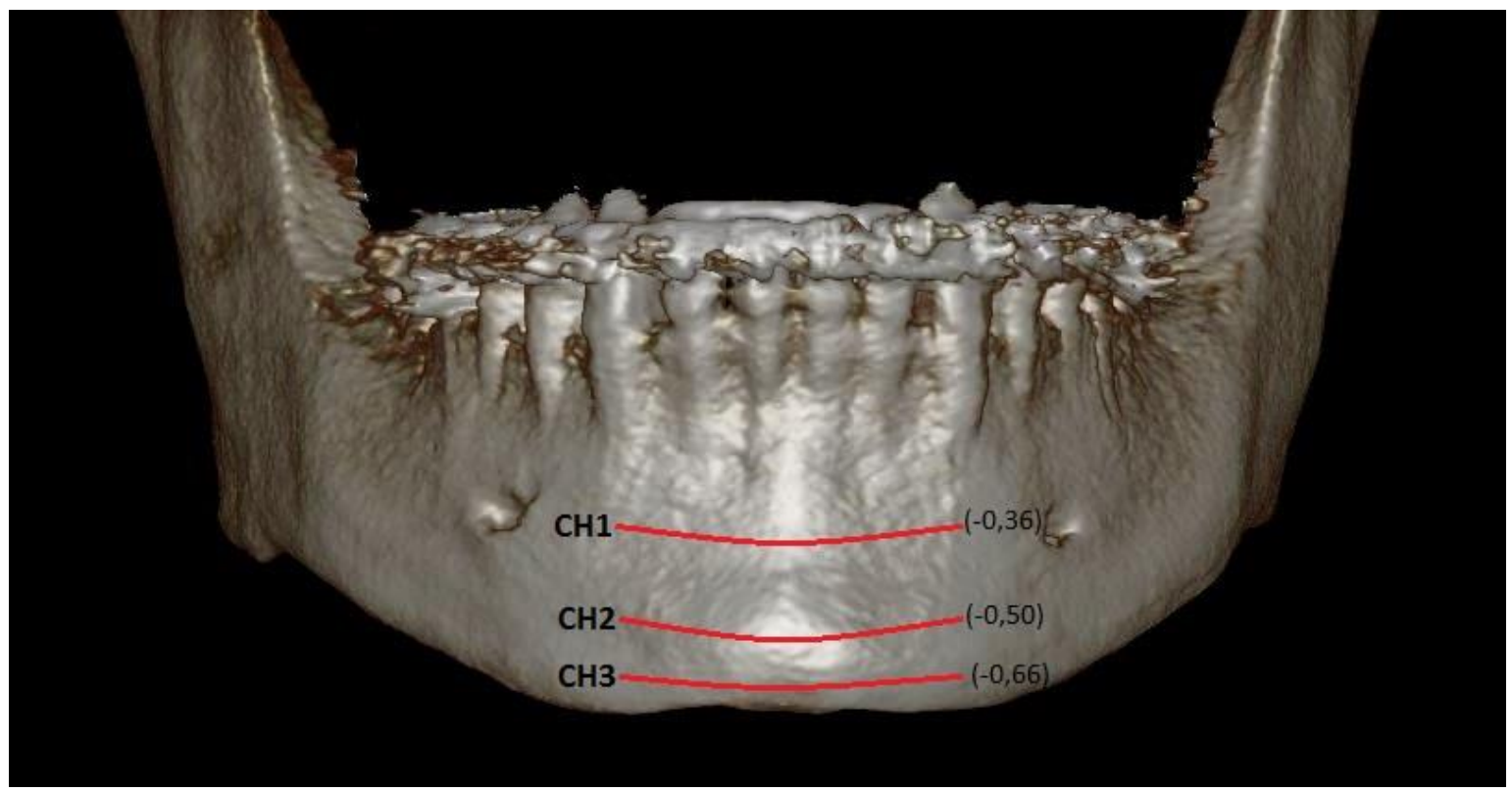

DVT-Rekonstruktion der Mandibula mit untersuchten Messpunkten (CH1 bis $\mathrm{CH} 3$ ) und den entsprechenden Pearson Korrelationen zu dem ML-NSL (Klinik für Mund-, Kiefer- und Gesichtschirurgie Universitätsklinikum Göttingen)

Durch die frühzeitige Verknöcherung der Symphysis mentalis innerhalb des ersten Lebensjahres, entfällt diese als langfristiges Wachstumszentrum. Das transversale Wachstum im Bereich des Kinns erfolgt in den anschließenden Lebensjahren deshalb vermehrt durch periostale Knochenapposition zum Beispiel am Unterrand des Kinns (Bjork 1963). Die Messung CH3 liegt in unmittelbarer Nähe dieser Wachstumsprozesse und scheint hiervon stärker beeinflusst zu werden als beispielsweise $\mathrm{CH} 1$. Interindividuelle Unterschiede des periostalen Wachstums könnten somit die höhere Korrelation und Standardabweichung für $\mathrm{CH} 3$ im Vergleich zu CH1 und $\mathrm{CH} 2$ erklären. Das bedeutet, dass bei einem vertikalen Wachstumsmuster (ML-NSL erhöht) die horizontale Dimension des Kinns in der Nähe der Basis (CH3) wahrscheinlich stärker reduziert ist, als in der Nähe des Alveolarfortsatzes (CH1). Hierdurch kann ein typisches, dolichofaziales Erscheinungsbild entstehen. Ein horizontales Wachstumsmuster hingegen (ML-NSL verringert), korreliert mit einem breiteren Kinn und größerem interforaminalem Abstand. 
Die drei vertikalen Messungen CV1 bis CV3 erstrecken sich vom Alveolarfortsatz bis zur Basis des Unterkiefers und sind somit gleichermaßen über diese beiden Wachstumszonen verteilt. Daher ist, den vorangehenden Überlegungen zufolge, eine geringere Diskrepanz zwischen den Standardabweichungen und Korrelationen zum ML-NSL zu erwarten als bei CS und $\mathrm{CH}$. Abbildung 62 stellt diesen Sachverhalt dar.

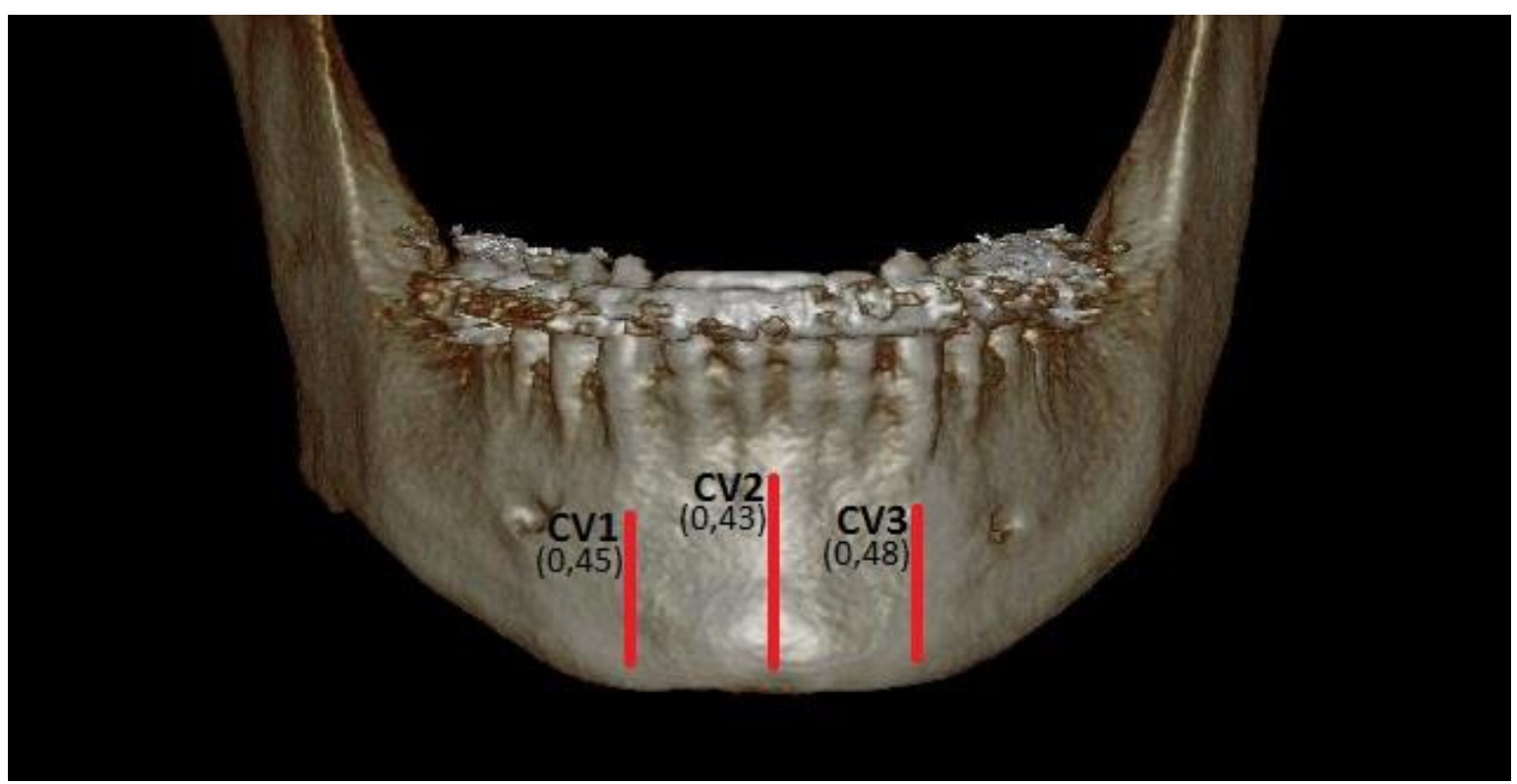

Abbildung 62

DVT-Rekonstruktion der Mandibula mit untersuchten Messungen (CV1 bis CV3) und den entsprechenden Pearson Korrelationen zu dem ML-NSL (Klinik für Mund-, Kiefer- und Gesichtschirurgie Universitätsklinikum Göttingen)

\subsubsection{Korrelationen zwischen FRS-Messerwerten und Knochendimensionen der Retromolarregion}

Die untersuchten Strecken und Volumina des retromolaren Knochenblockes weisen ebenfalls statistisch signifikante Korrelationen zu FRS-Messungen auf. Der ANB-Winkel korreliert positiv mit dem vertikalen retromolaren Knochenangebot (MN1 bis MN3), während SNB und Kieferwinkel hiermit negativ korrelieren. Sowohl ein vergrößerter ANB, als auch ein verkleinerter SNB können eine distale Bisslage beschreiben. Hierbei ist zu bedenken, dass der SNA und ML-NSL durch ihren Einfluss auf den ANB einen maskierenden Effekt ausüben können. Hierdurch kann die tatsächliche sagittale Kieferrelation möglicherweise nicht korrekt erfasst werden. Mit dem iANB soll dieser potentielle Effekt nivelliert werden (Panagiotidis und Witt 2005). Im Gegensatz zum ANB, weist der iANB jedoch keine statistisch signifikanten Korrelationen mit dem retromolaren Knochenvolumen auf. Auch das vertikale 
oder bukko-linguale retromolare Knochenangebot (MN1 bis MN3 bzw. MW1 bis MW9) korreliert nicht mit dem iANB. Dies legt nahe, dass durch den Einfluss des ML-NSL und SNA auf den ANB ein besserer Prädiktor für das retromolare Knochenangebot geschaffen wird als der iANB. ML-NSL und SNA per se weisen diese Korrelationen mit dem retromolaren Knochenangebot jedoch nicht auf.

Patienten mit distaler Bisslage, nach ANB und SNB sowie kleinem Kieferwinkel, könnten besonders interessant für die Entnahme retromolarer Knochenblöcke sein. Diese Konstellation korreliert mit einer vergrößerten Distanz zwischen kranialer Knochenoberfläche im retromolaren Bereich des Unterkiefers und Nervus alveolaris inferior. Außerdem liegt keine negative Korrelation mit der bukko-lingualen Knochendicke (MW) vor, sodass eine signifikant positive Korrelation zwischen dem ANB und retromolaren Knochenvolumen (Vretro) besteht (Tabelle 20). Möglicherweise entsteht durch die distale Bisslage nach ANB, SNB und einen kleineren Kieferwinkel eine Stauchung des retromolaren Raumes, die in einem vermehrten Knochenangebot resultiert (Abb. 63). Eine direkte Korrelation zwischen ANB und dem Kieferwinkel konnte nicht nachgewiesen werden, sodass sie unabhängig voneinander mit dem retromolaren Knochenangebot korrelieren. Ob eine solche Stauchung die mesiodistale Ausdehnung des retromolaren Knochenblockes eventuell reduziert, wurde im Rahmen dieser Arbeit nicht geprüft und könnte Gegenstand weiterer Untersuchungen sein.

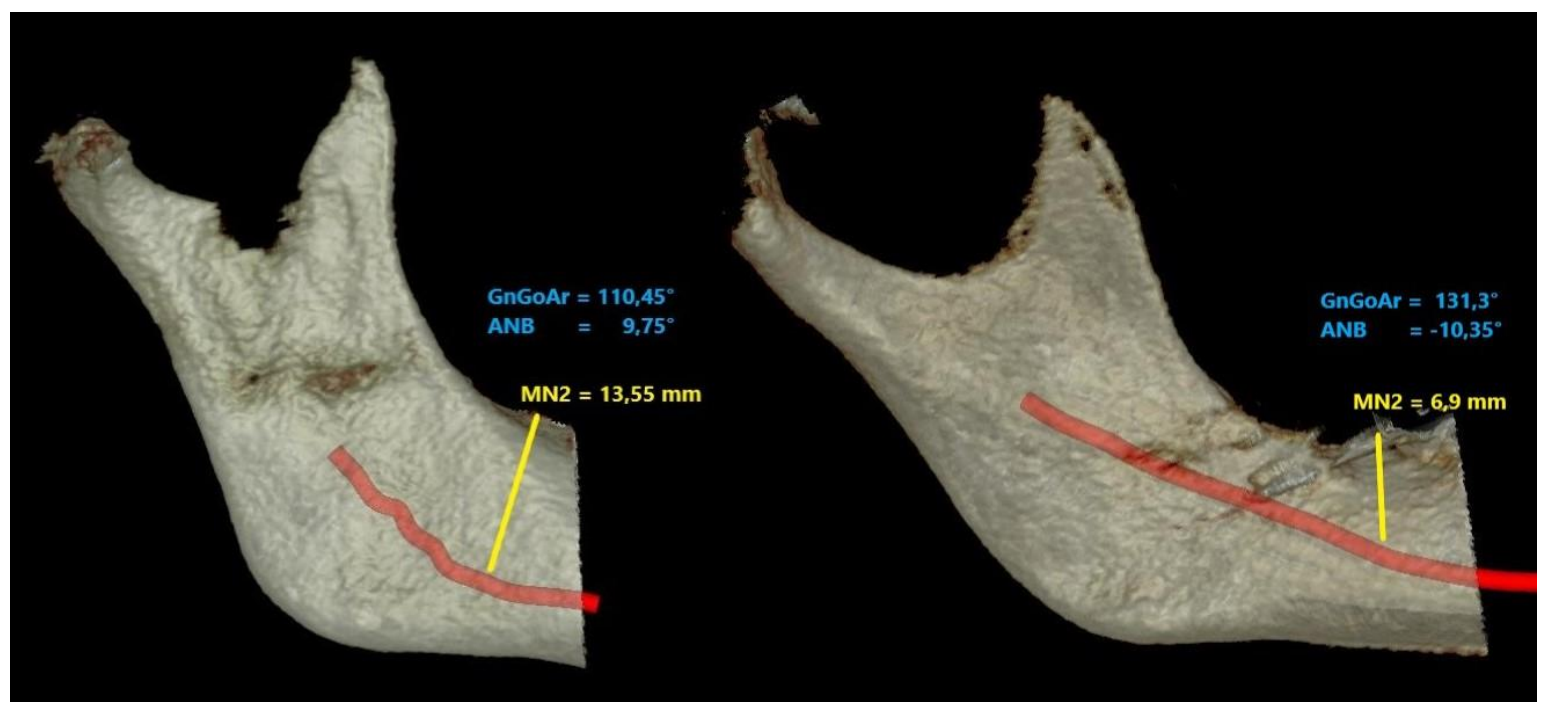
Beispiel mit erhöhtem retromolarem Knochenangebot bei distaler Bisslage nach ANB und kleinerem GnGoAr. Rechts ein Beispiel mit reduziertem retromolarem Knochenangebot bei mesialer Bisslage nach ANB und größerem GnGoAr. Der Nervus alveolaris inferior ist rot markiert. (Klinik für Mund-, Kiefer- und Gesichtschirurgie Universitätsklinikum Göttingen) 
Ältere Untersuchungen konnten keine Korrelationen zwischen ANB und Gesamtknochenvolumen der Mandibula nachweisen (Deguchi et al. 2010; Katayama et al. 2014). Bei der Untersuchung isolierter mandibulärer Knochenspenderregionen in der vorliegenden Arbeit konnten jedoch Korrelationen nachgewiesen werden. Dies legt nahe, dass die Knochenvolumina einzelner Bereiche der Mandibula mit kephalometrischen Parametern korrelieren, während andere Bereiche keine oder entgegengesetzte Korrelationen aufweisen.

Auffallend ist auch, dass die retromolare Knochendicke in bukko-lingualer Richtung auf halber Länge zwischen Knochenoberfläche und Nerv (MW2, MW5, MW8) durchschnittlich dicker ist, als in den kranialen (MW1, MW4, MW7) und kaudalen Messpositionen (MW3, MW6, MW9). Diese Differenz zwischen kranialen und kaudalen Knochendicken ist in den drei mesialen Messpositionen am stärksten ausgeprägt (MW1 < MW2 > MW3). Bei Beachtung der anatomischen Gegebenheiten wird ersichtlich, dass die lingual befindliche Linea mylohyoidea auf Höhe der durchschnittlich größer ausfallenden Messungen verläuft. Dies ist in den Abbildungen 16, 18 und 64 erkennbar. Auch die größere Differenz zwischen den drei mesialen Dickenmessungen lässt sich durch die Linea mylohyoidea erklären, die nach distal abflacht (Abb. 64).

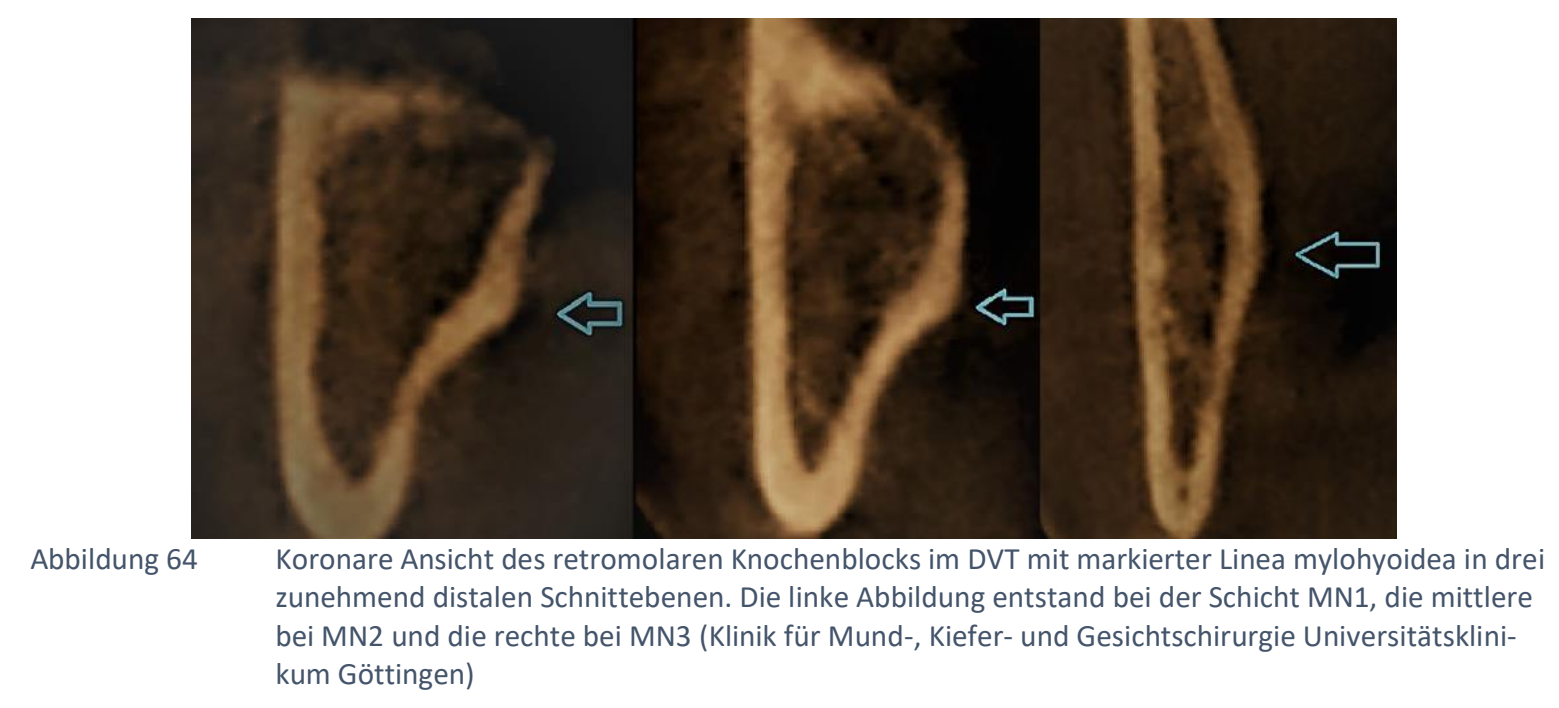

Bei der untersuchten Patientenpopulation verlief der Nervus alveolaris inferior doppelt so häufig in der lingualen Hälfte der retromandibulären Anteile der Mandibula wie in der bukkalen. Weitere Untersuchungen an DVT-Aufnahmen kamen ebenfalls zu dem Ergebnis, dass der Nervus alveolaris inferior signifikant häufiger nahe der lingualen als bukkalen Kortikalis der Mandibula verläuft (Levine et al. 2007; Khorshidi et al. 2017; Kalabalik und Aytuğar 2019). Bezüglich der bukko-lingualen Lage des Nervus alveolaris inferior, konnte in 
der Patientenpopulation der vorliegenden Arbeit eine bilaterale Symmetrie festgestellt werden. Ein Review kam zu dem Ergebnis, dass eine solche bilaterale Symmetrie des Nervverlaufs die Regel zu sein scheint (Anderson et al. 1991). Für den Verlauf des Nervus alveolaris inferior konnte auch ein Zusammenhang mit kephalometrischen Parametern nachgewiesen werden. Das Modell für eine logistische Regression mit backward removal Algorithmus ergab, dass eine Erhöhung des Hasund-Scores um 1 das Risiko, dass sich der Nerv an untersuchter Position in der bukkalen Hälfte der Mandibula befindet, um 18,6 \% erhöht. Folglich sind nach anterior rotierende Wachstumstypen öfter mit bukkalem als lingualem Verlauf des Nerven assoziiert. Bei der Entnahme retromolarer Knochenblöcke könnte bei Patienten mit anteriorem Rotationsmuster daher eher mit der bukkalen Lage des Nerven als limitierendem Faktor gerechnet werden. Im Rahmen sagittaler Spaltungen der Mandibula bei dysgnathiechirurgischen Eingriffen könnte diese Erkenntnis ebenfalls hilfreich sein, da eine reduzierte Distanz des Nerven zur bukkalen Kortikalis ein signifikant häufigeres Risiko für postoperative Sensibilitätsstörungen birgt (Rich et al. 2014). Aufgrund dieser klinischen Relevanz ist der bukko-linguale Nervverlauf Gegenstand vieler Untersuchungen. Bei einer longitudinalen Wachstumsanalyse anhand von CT-Aufnahmen des Schädels von Kindern konnte eine laterale Wanderung des Nervus alveolaris inferior in allen untersuchten Fällen beobachtet werden (Krarup et al. 2005). Ob diese laterale Wanderungstendenz bei anteriorem Wachstumsmuster stärker ausgeprägt ist und möglicherweise einem Stauchungseffekt unterliegt, wurde hingegen nicht untersucht. Bezüglich einer Korrelation zwischen Ausmaß der lateralen Wanderung während des Wachstums und dem kieferorthopädischen Wachstumsmuster bedarf es folglich weiterer Untersuchungen. Insgesamt scheinen skelettal distale Kieferrelationen nach ANB und anteriore Rotationsmuster mit günstigeren Bedingungen für die Entnahme retromolarer Knochenblöcke assoziiert zu sein. Während diese Wachstumsmuster mit erhöhtem, retromolarem Knochenvolumen und einer größeren Distanz zwischen okklusaler Knochenoberfläche und Nerv korrelieren, könnte die erhöhte Wahrscheinlichkeit für einen bukkal verlaufenden Nervus alveolaris inferior jedoch den operativen Zugang erschweren oder einschränken. 


\subsubsection{Korrelationen mit DVT-Messwerten der Crista zygomatico-alveolaris}

Die Knochendicken und -volumina der Crista zygomatico-alveolaris wiesen im Rahmen dieser Arbeit statistisch signifikante Korrelationen ausschließlich mit dem Knochenangebot der retromolaren Region auf (Tabelle 21). Während die retromolare Region ihrerseits Korrelationen mit FRS-Werten aufweist, trifft dies für die Crista zygomatico-alveolaris nicht zu. Diese Beobachtung könnte darin begründet sein, dass zumindest die Knochendicke der Crista zygomatico-alveolaris weniger mit kieferorthopädischen Wachstumsmustern, als mit charakteristischen Trajektorien bei Kaubelastungen korreliert. Als Teil des vertikalen Jochbeinpfeilers wird im Bereich der Crista zygomatico-alveolaris der Kaudruck übertragen und verteilt. Diese Hauptkraftlinien erstrecken sich sowohl über die Crista zygomatico-alveolaris, als auch über den retromolaren Bereich und insbesondere über die Linea obliqua (Abb. 65). Als Reaktion auf diese Krafteinwirkungen können entsprechende Verdickungen der Kompakta gebildet werden (Schünke et al. 2009). Andere Untersuchungen konnten nachweisen, dass ein stark ausgeprägter horizontaler Wachstumstyp mit erhöhter Kaukraft korreliert (Quiudini et al. 2017). Es konnte im Rahmen dieser Arbeit jedoch keine statistisch signifikante Korrelation zwischen dem Hasund-Score und den Knochendimensionen der Crista zygomatico-alveolaris nachgewiesen werden. Lediglich eine statistisch nicht signifikante, schwache Korrelation zwischen der Knochendicke an der Position ZA5 und dem Hasund-Score, könnte als Hinweis auf eine mögliche Tendenz interpretiert werden. Eine weitere Untersuchung, die nach Korrelationen zwischen Kaukraft und dem Knochenangebot der Crista zygomatico-alveolaris sucht, könnte hier neue Erkenntnisse schaffen.

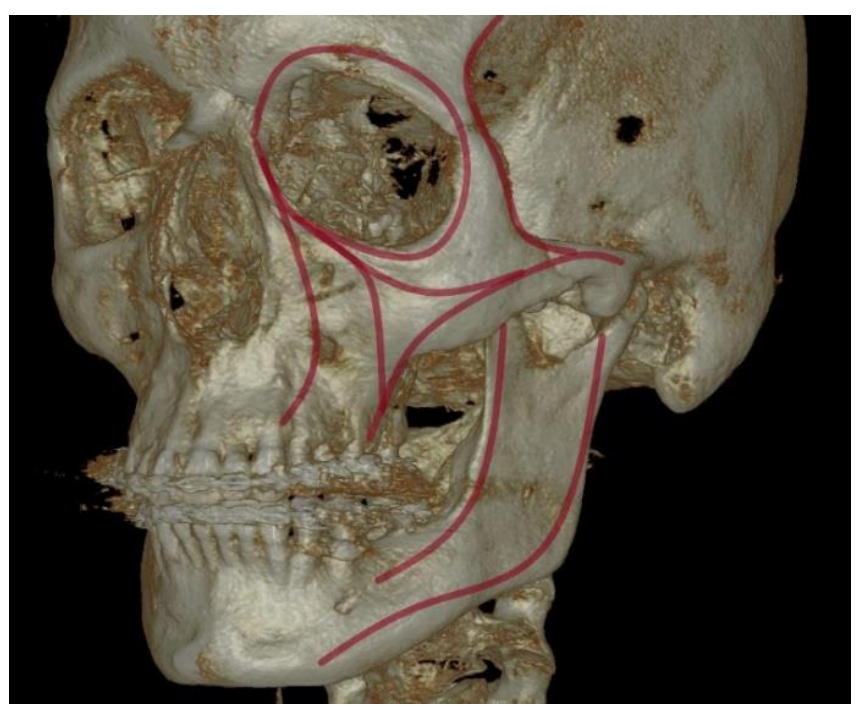




\subsubsection{Korrelationen zwischen OPG-Messwerten und Knochendimensionen im DVT}

\subsubsection{Korrelationen zwischen OPG-Messerwerten und Knochendimensionen des Kinns}

Im Bereich des Kinns besteht eine starke positive Korrelation der Messung WS1 im OPG mit den ebenfalls vertikalen Messungen CV1 bis CV3. Mit einem Korrelationskoeffizienten von 0,83 für WS1 und CV2 ist diese Korrelation den Erwartungen entsprechend stark, da es sich prinzipiell um die gleiche gemessene Strecke handelt. Die Tatsache, dass der Koeffizient nicht noch näher an einer perfekten Korrelation von 1,0 liegt, ist darin begründet, dass durch eine Neigung des Kopfes in der Sagittalebene und des Alveolarkammes eine reduzierte vertikale Dimension auf dem Röntgensensor des OPGs projiziert werden kann (Abb. 66).

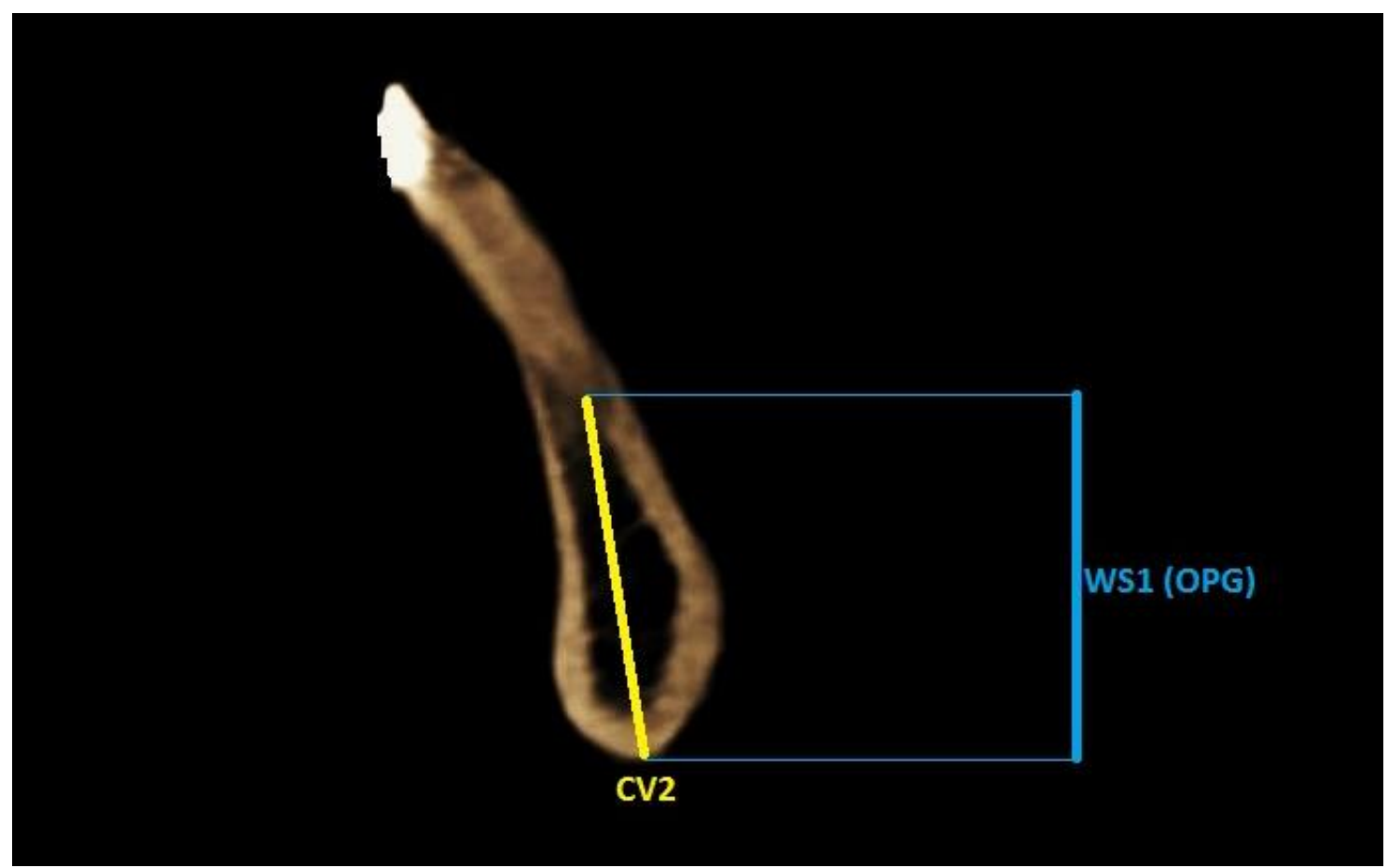

Abbildung 66

Sagittalschnitt durch den mittleren Unterkiefer Incisivus im DVT. Die im OPG erfasste Distanz zwischen Apex und Unterkieferbasis ist blau dargestellt. Der tatsächliche Abstand konnte in der DVTAufnahme gemessen werden und ist gelb dargestellt (Klinik für Mund-, Kiefer- und Gesichtschirurgie Universitätsklinikum Göttingen)

Die bereits erläuterten gegenläufigen Korrelationen der Kinndicke (CS) und Kinnlänge (CV) ließen sich auch im OPG nachweisen. WS1 korreliert negativ mit der Kinndicke (CS). Auch wenn im OPG die sagittale Dicke des knöchernen Kinns nicht direkt beurteilt werden kann, korreliert eine geringe Distanz zwischen der Wurzelspitze der mittleren Incisivi und der Basis des Kinns (WS1) mit einem ausgedehnten Kinn in sagittaler Richtung (CS). Bei einem 
vergrößerten WS1 im OPG hingegen, ist bei der Entnahme eines Knochenblockes die tendenziell reduzierte Distanz zur lingualen Kortikalis zu beachten.

Eine weitere signifikante Korrelation besteht zwischen dem WS1 der OPG-Aufnahme und dem gesamten entnehmbaren Knochenvolumen des Kinns (Vchin). Ein großer WS1 korreliert mit einem großen Knochenvolumen im Kinn (Tabelle 18). Da WS1 allerdings zugleich negativ mit der sagittalen Knochendicke des Kinns (CS) korreliert, sollte auch hier die tendenziell reduzierte Distanz zur lingualen Kortikalis bei der Transplantatentnahme beachtet werden (Tabelle 18).

Abgesehen von der Evaluation des Knochenblockausmaßes im Kinn gibt es noch weitere Gründe für die Anfertigung präoperativer DVT-Aufnahmen. Die Autoren einiger Veröffentlichungen raten aufgrund des sehr häufigen Vorkommens des Canalis incisivus im interforaminalen Bereich und dem damit verbundenen Komplikationsrisiko für postoperative Sensibilitätsstörungen zu routinemäßigen präoperativen DVT-Aufnahmen bei Eingriffen im Bereich der Kinnsymphyse. Der Nachweis eines solchen interforaminalen Kanals gelang in diesen Untersuchungen bei 92 bis 100 \% der DVT-Aufnahmen (Pereira-Maciel et al. 2015; Zeltner et al. 2016; Kabak et al. 2017). Das Auffinden dieser Struktur mittels OPG-Aufnahme gelang in einer Untersuchung bei lediglich $11 \%$ der untersuchten Aufnahmen. Das OPG ist in diesem Aspekt dem DVT deutlich unterlegen (Pires et al. 2012)

\subsubsection{Korrelationen zwischen OPG-Messerwerten und Knochendimensionen der Retromolarregion}

Die Distanz zwischen den Apices des zweiten Unterkiefer Molaren zur Basis des Unterkiefers (WS7, Abb. 11) korreliert mit dem retromolaren Knochenvolumen (Vretro). Möglicherweise wird mit WS7, analog zu MN, die vertikale Dimension im retromolaren Bereich erfasst. Diese ist maßgeblich am Knochenvolumen beteiligt.

Obwohl WS7 mit Vretro und Vretro wiederum mit Vcrista korreliert, liegt keine Korrelation zwischen WS7 und Vcrista vor. WS7 erfasst zwar ansatzweise die vertikale Dimension des retromolaren Knochenvolumens, ist aber möglicherweise im Gegensatz zu den drei Dimensionen des retromolaren Knochenblocks und der Crista zygomatico-alveolaris nicht gleich- 
ermaßen an Adaptationsprozessen durch Kaubelastungen beteiligt. Diese Prozesse betreffen viel mehr die laterale Kompakta der Mandibula, sodass WS7 als vertikaler Wert womöglich weniger betroffen ist. Die Messungen an den OPG-Aufnahmen weisen keine statistisch signifikanten Korrelationen mit den DVT-Messungen an der Crista zygomatico-alveolaris auf.

Die Korrelation zwischen den Kieferwinkeln, gemessen in OPG- und FRS-Aufnahmen, ist mit einem Korrelationskoeffizienten von $r=0,89$ sehr stark (Tabelle 20). Analog zum Kieferwinkel in FRS-Aufnahmen, besteht daher auch eine negative Korrelation zwischen dem Kieferwinkel in OPG-Aufnahmen und dem vertikalen Knochenangebot der retromolaren Entnahmeregion (MN). Ein großer Kieferwinkel in OPG-Aufnahmen korreliert mit geringeren Entfernungen zwischen okklusaler Knochenoberfläche und Nervus alveolaris inferior (MN). Eine Messung dieser Dimension ist in OPG-Aufnahmen zwar möglich ohne den Kieferwinkel zu bestimmen, jedoch kann die Lage des Nervus alveolaris inferior und die Neigung des Alveolarkammes in bukko-lingualer Richtung in OPG-Aufnahmen nicht erkannt werden. Die

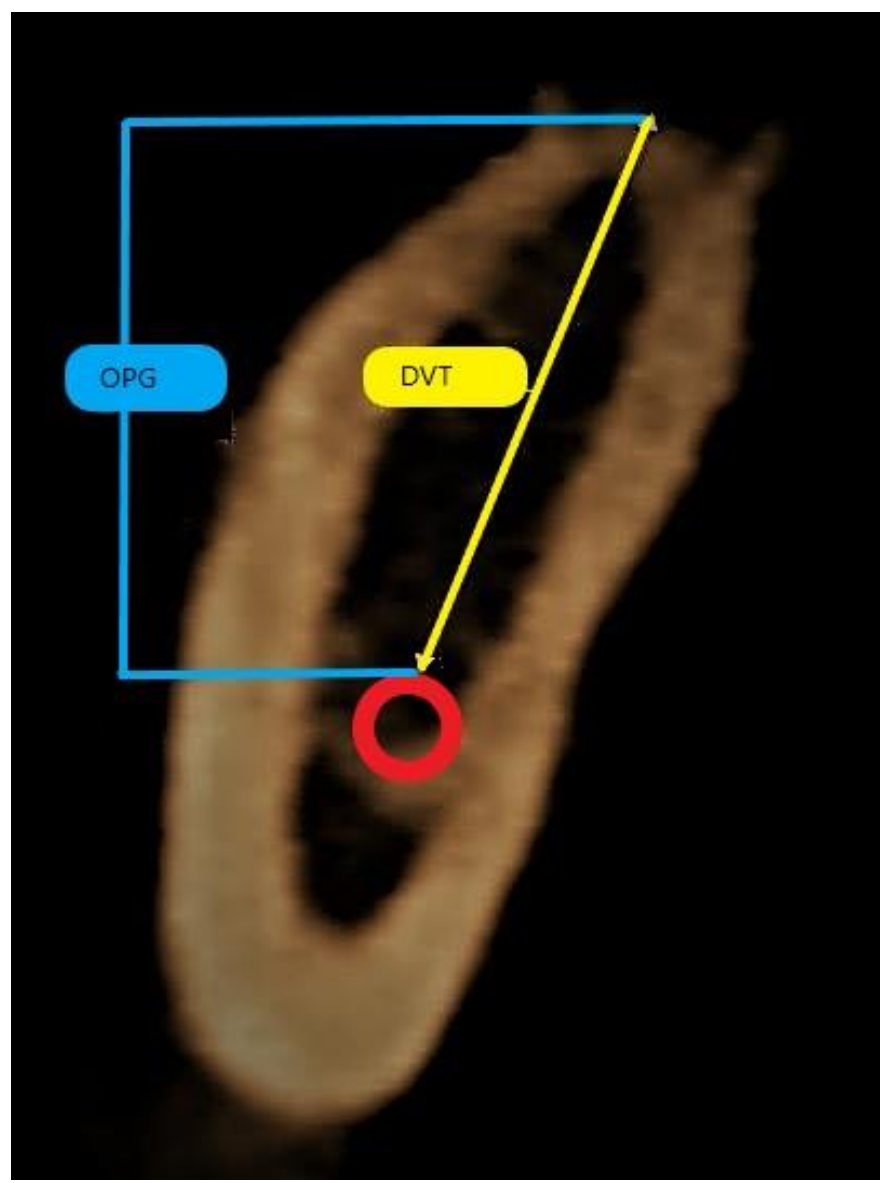
im OPG erfasste Distanz zwischen okklusaler Knochenoberfläche und Nervus alveolaris inferior ist blau dargestellt. Der tatsächliche Abstand konnte in der DVT-Aufnahme gemessen werden und ist gelb dargestellt (Klinik für Mund-, Kiefer- und Gesichtschirurgie Universitätsklinikum Göttingen) 
Korrelation zwischen dem Kieferwinkel in OPG-Aufnahmen und dem tatsächlichen Abstand zwischen Nerv und okklusaler Knochenoberfläche (MN), erlaubt eine ergänzende Abschätzungsmöglichkeit neben der direkten Messung der vertikalen, retromolaren Knochendimension an der OPG-Aufnahme. In DVT-Aufnahmen hingegen ist es möglich den tatsächlichen Abstand zwischen Nerv und okklusaler Knochenoberfläche (MN) zu messen (Abb. 67). Vorangehende Untersuchungen konnten jedoch nachweisen, dass die Differenzen zwischen Messungen des vertikalen retromolaren Knochenangebotes an OPG- und DVT-Aufnahmen nicht statistisch signifikant sind (Amarnath et al. 2015). 


\subsubsection{Korrelationen zwischen OPG- und FRS-Messwerten}

Die Kieferwinkelmessungen im OPG und FRS weisen eine starke, positive Korrelation miteinander auf. Dieses Ergebnis deckt sich mit zuvor durchgeführten Untersuchungen (Okşayan et al. 2012; Zangouei-Booshehri et al. 2012; Radhakrishnan et al. 2017). Das OPG hat gegenüber dem FRS hierbei den Vorteil, dass keine Doppelkonturen von Kieferwinkel, -kondylen oder -basen dargestellt werden. Während der Analyse des Kieferwinkels im OPG kann der Behandler entweder eine Seite ausgrenzen oder den Mittelwert beider bilden. Zweiteres entspräche dem üblichen Vorgehen im FRS. Im Gegensatz zum FRS dient das OPG jedoch primär der übersichtlichen Darstellung der gesamten Dentition, während ein Großteil der anderen FRS-Variablen nicht im OPG erfasst werden kann. Das FRS hingegen wird umfassenden kephalometrischen Fragestellungen gerecht. Für die Verlaufs- und Erfolgskontrolle einer kieferorthopädischen Therapie ist daher das FRS üblich.

\subsubsection{Vergleich der Knochenspenderregionen}

Die gemessenen durchschnittlichen Knochenvolumina der drei untersuchten Areale unterscheiden sich stark voneinander (Abb. 48). Während im Kinn durchschnittlich das größte Volumen vorliegt, verfügt die Crista zygomatico-alveolaris über das kleinste Knochenvolumen. Neben dem Knochenvolumen können allerdings noch andere Eigenschaften die Entscheidung des Chirurgen bei der Auswahl der Spenderregion beeinflussen.

So könnte die Crista zygomatico-alveolaris trotz des sehr geringen Knochenvolumens als Entnahmeareal in Frage kommen. Durch ihre besonders in horizontaler Richtung ausgeprägte Krümmung, könnte sie für die Anwendung bei Augmentationen im anterioren, ästhetisch relevanten Bereich des Oberkiefers in Frage kommen (Kainulainen et al. 2002). Eine sekundäre Weichgewebstransplantation zur Wiederherstellung der alveolären Ästhetik ist in diesen Fällen seltener nötig (Stübinger et al. 2006; Gellrich et al. 2007; Gellrich et al. 2013). Kleinere Defekte wie freiliegende Implantatgewinde könnten ebenfalls versorgt werden (Kainulainen et al. 2002). Die Anwendung piezoelektrischer Techniken reduziert hierbei die Entnahmemorbidität (Stübinger et al. 2006; Stübinger et al. 2015). So wird durch 
ein piezochirurgisches Vorgehen das Risiko einer Verletzung der Schneider-Membran erheblich reduziert (Kainulainen et al. 2002). Ein ausreichender Sicherheitsabstand zum Foramen infraorbitale ist gut einzuhalten, sodass postoperative Sensibilitätsstörungen selten auftreten (Gellrich et al. 2013). Relevanter als das Volumen könnte die Knochendicke sein. Diese unterscheidet sich an den neun gemessenen Punkten (ZA1 - ZA9) erheblich und beträgt im Durchschnitt 1,65 mm. Messungen an humanen Schädelpräparaten ergaben einen durchschnittlichen Wert von 2,1 mm (Yates et al. 2013). Die Differenz ist vermutlich damit zu erklären, dass in der Untersuchung von Yates et al. (2013) keine Mehrpunktmessung wie in der vorliegenden Arbeit vorgenommen wurde. Stattdessen wurde ausschließlich an dem dicksten Punkt der Transplantate gemessen. Würde man diese Methode bei der hier untersuchten Patientenpopulation anwenden, käme man zu einem ähnlichen Ergebnis. Der Tabelle 8 ist zu entnehmen, dass für den Messpunkt ZA6 eine durchschnittliche Knochendicke von 2,08 $\pm 0,57 \mathrm{~mm}$ gemessen wurde.

Im Kinn wurde für die untersuchte Patientenpopulation ein durchschnittliches Knochenvolumen von $3,10 \mathrm{~cm}^{3}$ mit einer Standardabweichung von $\pm 1,11 \mathrm{~cm}^{3}$ errechnet. Publikationen bei denen das Kinn ebenfalls als potentielles Knochenspende-Areal untersucht wurde, kamen mit 3,5 $\pm 1,3 \mathrm{~cm}^{3}$ auf vergleichbare Ergebnisse (Zeltner et al. 2016). Die Messmethode dieser Arbeitsgruppe unterschied sich dadurch, dass keine multiplen, separaten Messungen der drei Dimensionen mit anschließender manueller Multiplikation erfolgten. Stattdessen ermittelten die Untersucher via Software das Knochenvolumen direkt. Während diese Methode mit einem geringeren Arbeits- und Zeitaufwand verbunden ist, erlangt man keine Informationen über die Zusammensetzung oder Verteilung des Volumens. Im Rahmen operativer Eingriffe zur Knochenentnahme könnten Strecken dem Volumen aber überlegen sein. Insbesondere wenn es darum geht, sensible Nachbarstrukturen wie den Nervus mentalis, die Wurzelspitzen oder die linguale Kortikalis zu schonen. Eine Aufklärung des Patienten über die Entnahmemorbidität ist unerlässlich. Hierzu gehören postoperative Sensibilitätsstörungen der Weichgewebsregionen und Mucosa des Kinns, sowie der Unterkiefer Incisivi (Clavero und Lundgren 2003; Nkenke und Neukam 2014). Zudem besteht das Risiko einer Veränderung der Kontur des Kinns, als Folge von Knochenregenerationsprozessen mit Bildung von Konkavitäten (Weibull et al. 2009; Nkenke und Neukam 2014). Entnahmetechniken, welche durch das Belassen eines zentralen, vertikalen Kompakta-Steges diese Kontur schonen sollen, haben den Nachteil, dass ein geringeres Knochenvolumen 
entnommen werden kann (Hahn et al. 2008b). Eine weitere mögliche Komplikation ist die Kinnptose. Diese kann als Resultat der horizontalen Schnittführung und Durchtrennung des M. mentalis entstehen. Falls die Stümpfe der kranialen und kaudalen Anteile des durchtrennten M. mentalis nicht wieder aneinandergenäht werden, kann durch den ausbleibenden vertikalen Muskelzug das Weichgewebe des Kinns herabsinken. Das hierdurch veränderte Erscheinungsbild des Patienten wird oft als unvorteilhaft empfunden, sodass weitere korrigierende chirurgische Maßnahmen die Folge sein können (Rubens und West 1989; Altiparmak et al. 2017). Diese Vielzahl an möglichen Komplikationen scheint laut einer Untersuchung ein möglicher Grund dafür zu sein, dass Patienten andere Knochenspenderregionen bevorzugen (Nkenke und Neukam 2014). Hierzu gehört unter anderem die Retromolarregion.

Das durchschnittliche retromolare Knochenvolumen der untersuchten Patientenpopulation liegt bei $1,66 \pm 0,54 \mathrm{~cm}^{3}$. Dieses Ergebnis ist ebenfalls mit vorherigen Publikationen vergleichbar, in denen ein durchschnittliches Volumen von $1,8 \pm 1,1 \mathrm{~cm}^{3}$ ermittelt wurde (Zeltner et al. 2016). Das höhere durchschnittliche Volumen könnte mit der Patientenpopulation und Methodik der genannten Arbeitsgruppe erklärt werden. Diese bestand zu einem Anteil aus Patienten mit distal verkürzten Zahnreihen, die infolgedessen mehr retromolaren Knochen zur Entnahme vorweisen konnten. Die mesio-distale Länge des Knochenblocks wurde in der Untersuchung von Zeltner et al. (2016) nicht für alle Transplante gleichermaßen festgelegt. Daher wurden bei Patienten mit distal verkürzter Zahnreihe größere Volumina gemessen. Die größere Standardabweichung ist ein weiterer Hinweis auf diesen Zusammenhang. Beim Betrachten der Knochenvolumina in dieser Region ist zu bedenken, dass bei Bedarf die kontralaterale Retromolarregion ebenfalls zur Knochengewinnung herangezogen werden kann. Hierdurch verdoppelt sich das entnehmbare Knochenvolumen in etwa (Zeltner et al. 2016). Während bei der Entnahme von Knochenblöcken, beispielsweise mit Trepanbohrungen, eher das Knochenvolumen von Interesse sein dürfte, stehen bei der Entnahme von Kompakta-Schalen die linearen Strecken der vestibulären Knochenwand im Vordergrund. Besonders Patienten mit distal verkürzten Zahnreihen könnten für die Entnahme von Kompakta-Schalen geeignet sein, da das Knochenvolumen durch die lokale Atrophie reduziert wird, während die mesio-distale Ausdehnung des entnehmbaren Knochenblockes aufgrund des fehlenden Molaren zunimmt (Schropp et al. 2003; Zeltner et al. 2016). Zusätzlich könnte der Wachstumstyp des Patienten bei der Entscheidungsfindung 
eine Rolle spielen. Während eine distale Bisslage und ein kleiner Kieferwinkel positiv mit dem retromolaren Knochenvolumen korrelieren und sich daher besonders gut für die Gewinnung von voluminösen Knochenblöcken eignen können, ließen sich bei Patienten mit mesialer Bisslage und großem Kieferwinkel möglicherweise längere Kompakta-Schalen aufgrund der mesio-distalen Streckung der Retromolarregion gewinnen. Wie auch beim Kinn gilt es in der Retromolarregion sensible Nachbarstrukturen zu schonen, um Blutungen oder postoperative Sensibilitätsstörungen zu vermeiden. Der N. alveolaris inferior, N. lingualis und die an das Entnahmegebiet angrenzenden Zähne stehen hierbei im Vordergrund. Insgesamt wird die Morbidität bei Knochenentnahmen aus der Retromolarregion des Unterkiefers jedoch als relativ gering beschrieben (Capelli 2003; Nkenke und Neukam 2014).

In der vorliegenden Arbeit wurde ein möglicher Einfluss des Geschlechts auf die Knochenvolumina der jeweiligen Spenderregionen untersucht. Abbildung 48 stellt die durchschnittlichen Knochenvolumina in Abhängigkeit vom Geschlecht dar. In der untersuchten Population ließen sich keine statistisch signifikanten Unterschiede der Knochenvolumina in Abhängigkeit vom Geschlecht nachweisen. Zudem hat das Geschlecht keinen Einfluss auf die Korrelationen zwischen kephalometrischen Parametern und den untersuchten Knochenvolumina. Lediglich die Kinnbreite an den Positionen $\mathrm{CH} 2$ und $\mathrm{CH} 3$ ist bei den untersuchten männlichen Patienten statistisch signifikant größer als bei den untersuchten Frauen. Mit ähnlichen Methoden wie in der vorliegenden Arbeit wurde in einer brasilianischen Studie unter anderem die Kinnlänge (analog zu CV2), die Kinndicke (analog zu CS) und die bimentale Breite (analog zu CH) an humanen Schädelpräparaten untersucht. Auch hier konnte für die vertikale und sagittale Dimension des Kinns kein statistisch signifikanter Unterschied zwischen den beiden Geschlechtern festgestellt werden. Wie auch in dieser Arbeit konnte für die Breite des Kinns jedoch ein statistisch signifikanter Unterschied zwischen männlichen und weiblichen Präparaten bzw. Patienten nachgewiesen werden (Lopez-Capp et al. 2017). Es lässt sich festhalten, dass die Breite des Kinns als einziger geschlechtsabhängiger Faktor nicht ausreicht, um eine Geschlechtsabhängigkeit für das gesamte Knochenvolumen im Kinn zu erwirken, da mit der vertikalen Länge (CV) und sagittalen Dicke (CS) des Kinns zwei weitere Faktoren des Volumens gegeben sind, die keine Korrelation mit dem Geschlecht aufweisen. 
Signifikante Unterschiede im entnehmbaren Knochenvolumen zwischen den untersuchten Entnahmeregionen beeinflussen die Entscheidung des Chirurgen in Bezug auf die Spenderregion. Zusätzliche Faktoren wie die Lage und Form des Knochendefektes in der Empfängerregion sowie individuelle anatomische Variationen, die einen Einfluss auf die Entnahmemorbidität haben können, sollten dabei jedoch nicht außer Acht gelassen werden (Silva et al. 2006). 


\section{Zusammenfassung}

Präimplantologische Knochenaugmentationen zum Aufbau von Knochendefekten nehmen eine zentrale Rolle in der zahnärztlichen Chirurgie ein. Neben körperfremden Knochenaugmentationsmaterialien wird dabei Gebrauch von autogenen Transplantaten gemacht. Das Auffinden und Evaluieren dieser im ambulanten Bereich oftmals intraoral gelegenen Spenderregionen, ist eine wichtige Voraussetzung für den Therapieerfolg. Neben direkten, klinischen Untersuchungsmethoden am Patienten stehen dem Zahnarzt radiologische Hilfsmittel zur Verfügung. Hierbei wird der Patient stets einer Strahlenbelastung ausgesetzt, sodass der Behandler sich eines diagnostisch wertvollen Erkenntnisgewinns möglichst sicher sein sollte. Im Rahmen einer implantologischen Erstberatung liegen zumeist OPG-Aufnahmen vor. FRS-Aufnahmen sind insbesondere bei Patienten mit voriger kieferorthopädischer Therapie bereits vorhanden und können bei der anschließenden implantologischen Versorgung von Patienten mit Nichtanlagen hilfreich sein. Der zusätzliche Gebrauch dreidimensionaler Bildgebung sollte demzufolge gut überlegt werden und gezielt erfolgen. Im Rahmen dieser Arbeit konnte festgestellt werden, dass einige kephalometrische Parameter der Fernröntgenseitenanalyse und Strecken bzw. Winkel in OPG-Aufnahmen signifikante Korrelationen mit den gemessenen Knochendimensionen in oft aufgesuchten intraoralen Knochenspenderregionen aufweisen. Patienten mit vertikalem Wachstumstyp haben im Bereich des Kinns ein erhöhtes vertikales Knochenangebot zwischen den Apices der Incisivi und der Unterkieferbasis. Zugleich ist bei solchen Patienten mit einem reduzierten interforaminalem Abstand zu rechnen. In sagittaler Richtung ist das Kinn in solchen Fällen außerdem tendenziell dünner, woraus ein reduzierter Abstand zur lingualen Kortikalis resultiert. Für die retromolaren Spenderregionen konnten ebenfalls signifikante Korrelationen nachgewiesen werden. Eine distale Bisslage nach ANB korreliert positiv mit dem retromolaren Knochenvolumen. Sowohl eine distale Bisslage als auch ein verkleinerter Kieferwinkel korrelieren mit einem vergrößerten Abstand zwischen retromolarer Knochenoberfläche und Nervus alveolaris inferior. Horizontale Wachstumsmuster korrelieren mit einem Verlauf des Nervus alveolaris inferior in der bukkalen Hälfte der Mandibula. Da ein bukkaler Verlauf des Nerven mit erhöhten postoperativen Beschwerden in Form von Sensibilitätsstörungen korreliert, ist bei horizontalem Wachstumsmuster ein entsprechend behutsames Vorgehen bei der Entnahme von Knochen aus der Retromolarregion zu empfehlen. Ein präoperatives DVT 
kann genauere Informationen über die Lage des Nerven liefern. Das Knochenangebot der Crista zygomatico-alveolaris weist keine Korrelationen mit den Messungen an OPG- und FRS-Aufnahmen auf und korreliert lediglich mit dem retromolaren Knochenvolumen. Bei der Untersuchung geschlechtsspezifischer Unterschiede der Knochenvolumina konnten keine statistisch signifikanten Korrelationen nachgewiesen werden. Vorhandene Korrelationen zwischen Knochenvolumina und kephalometrischen Parametern zeigen keine Abhängigkeit vom Geschlecht. Der interforaminale Abstand hingegen ist bei der untersuchten männlichen Population signifikant größer. Die gemessenen Korrelationen zwischen zweidimensionalen Parametern und Knochendimensionen könnten als Anhaltspunkte bei individuellen Erstberatungen und Untersuchungen von Patienten fungieren. Dem Behandler könnten sie außerdem als Hinweise für eine gezielte und volumenreduzierte Anwendung dreidimensionaler Bildgebung dienen. Dies kann zu einer reduzierten Strahlenbelastung und zur Befundung kleinerer Bildvolumina beitragen. 


\section{Datenanhang}

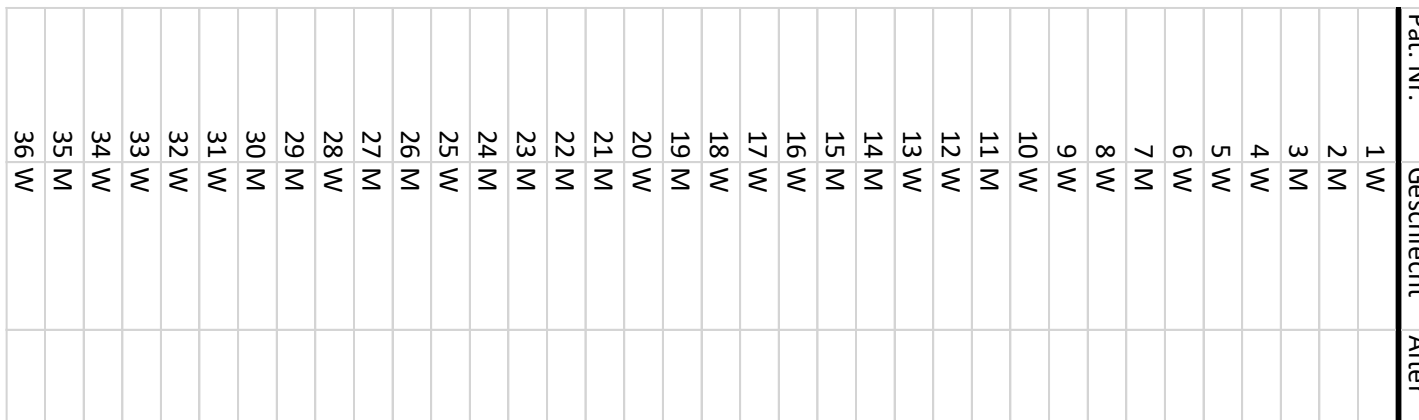

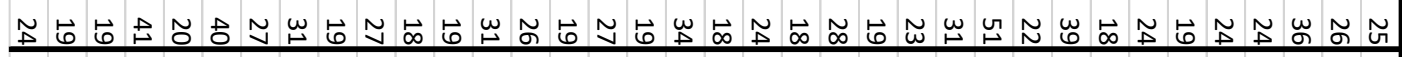

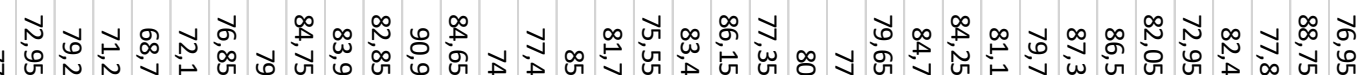

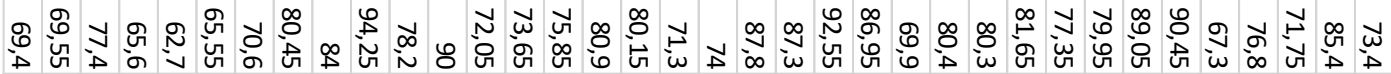

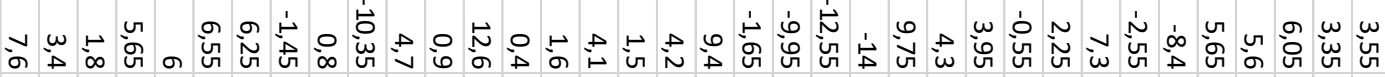

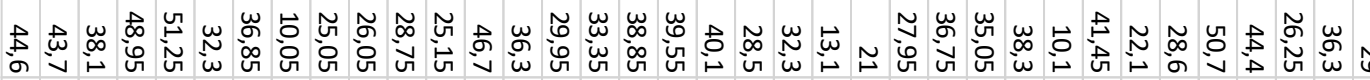

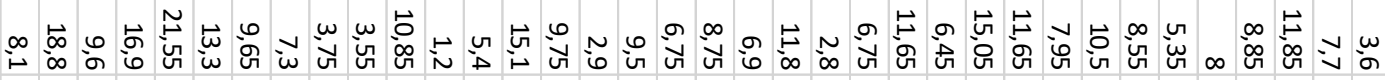

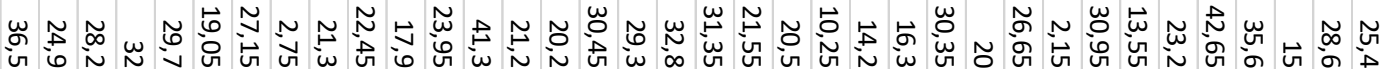

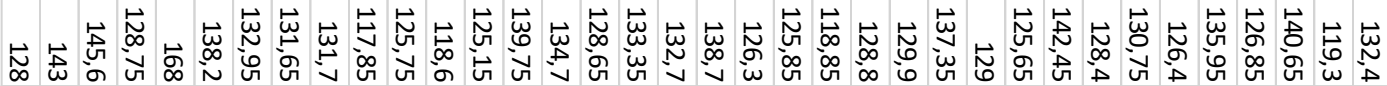

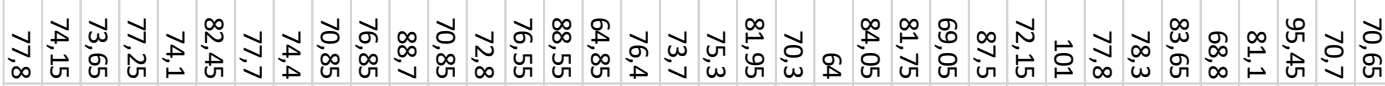

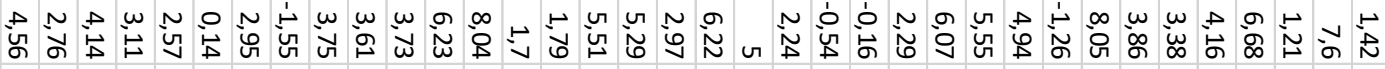

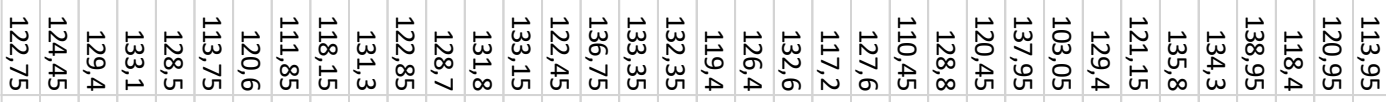

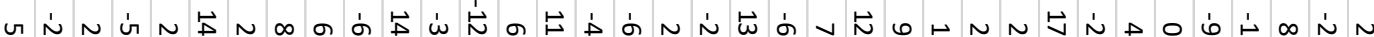




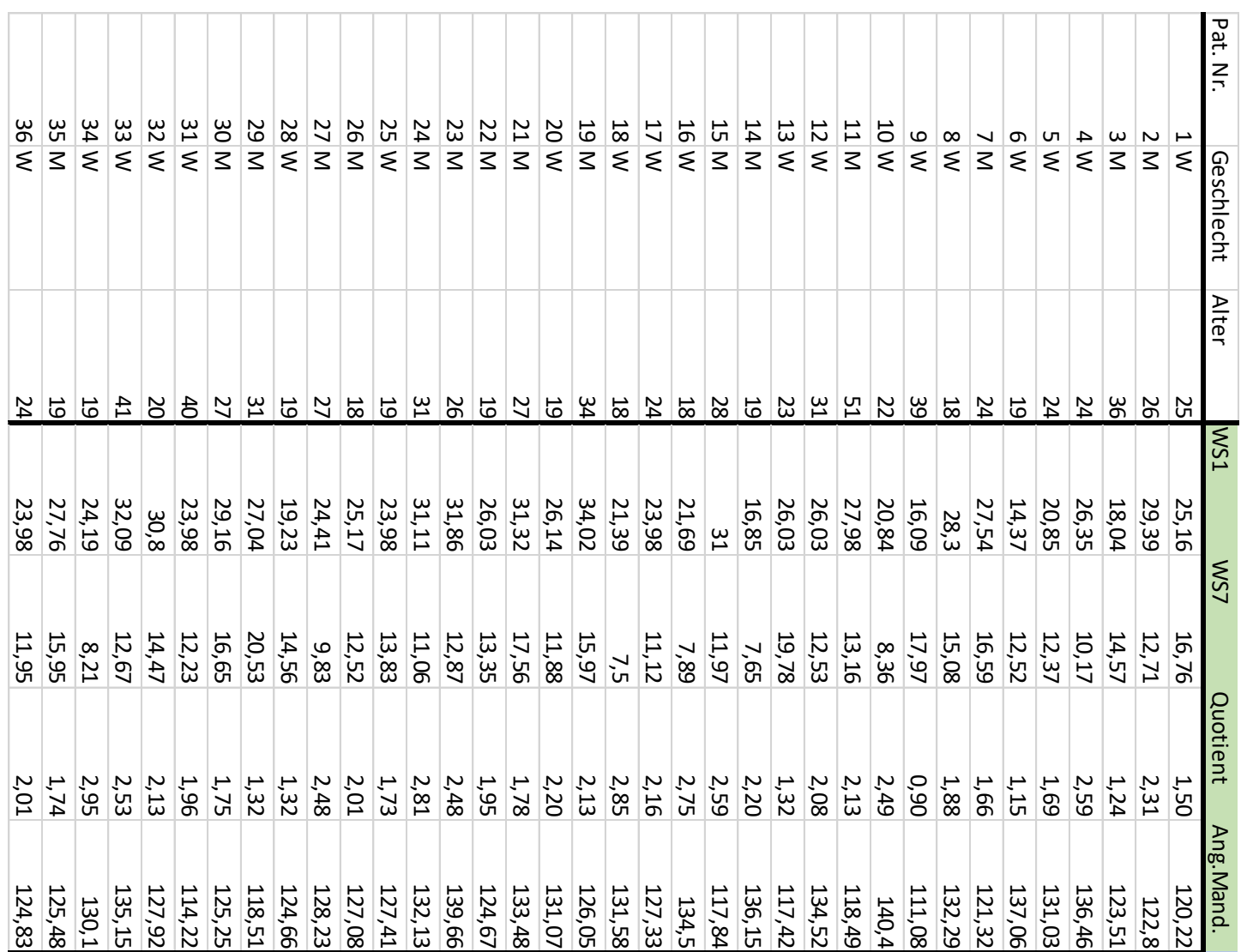

Tabelle A. 2

Tabellarische Darstellung der in OPG-Aufnahmen gemessenen Werte 


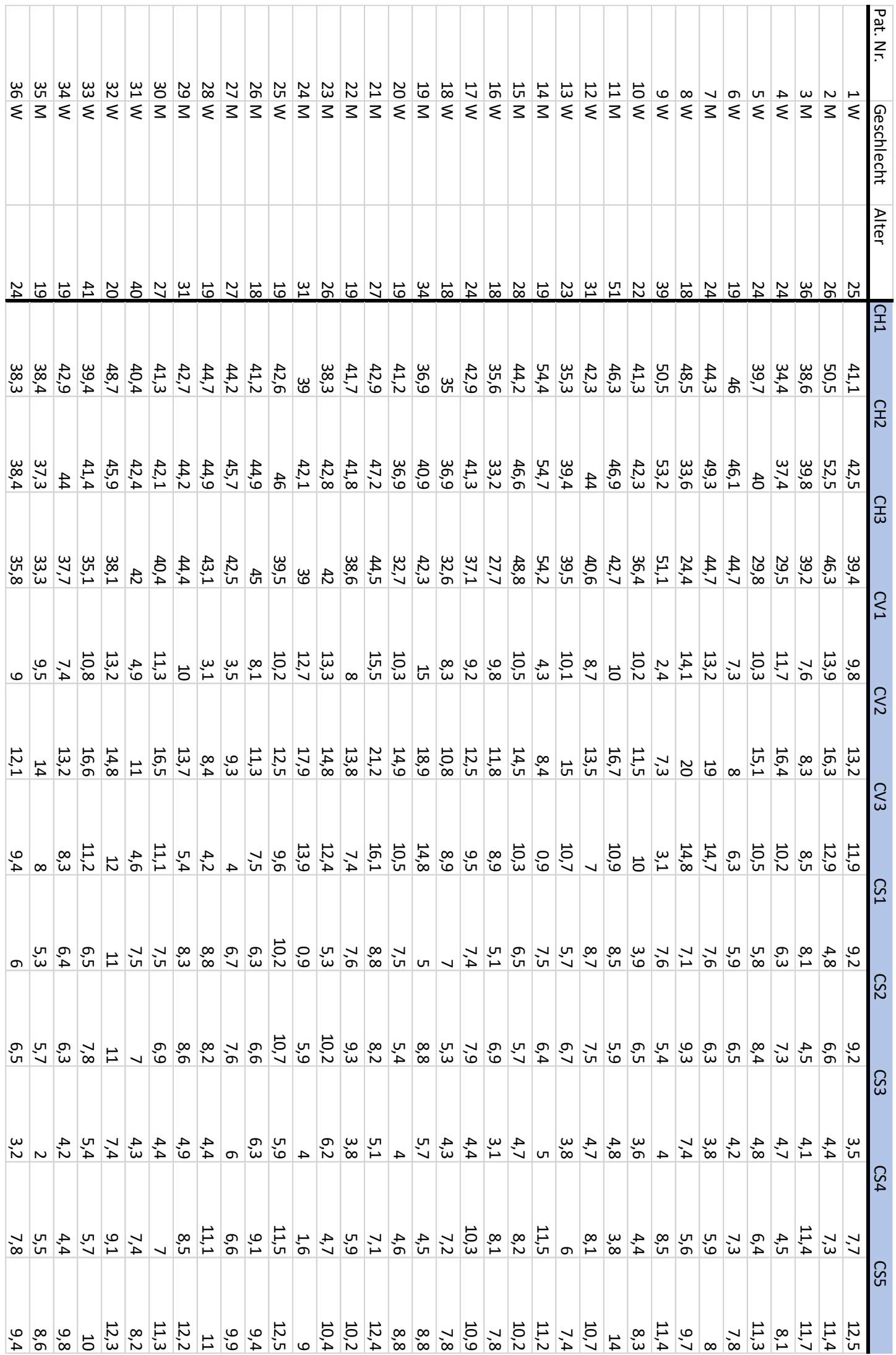

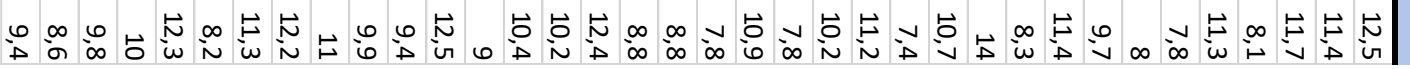

Tabelle A.3 Tabellarische Darstellung der in DVT-Aufnahmen gemessenen Werte 


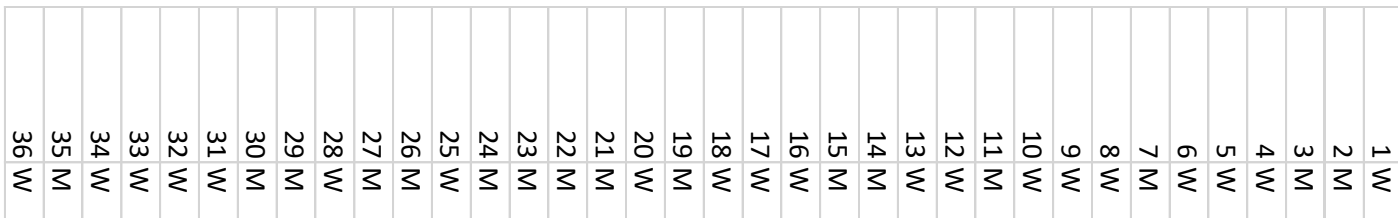

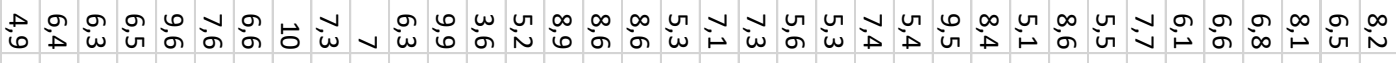

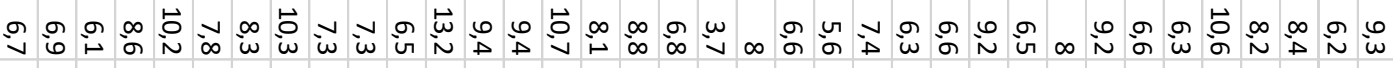

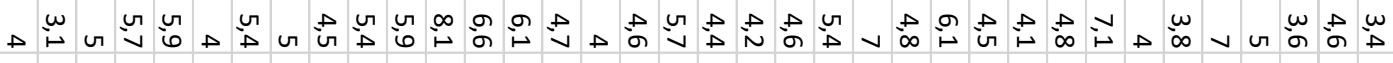

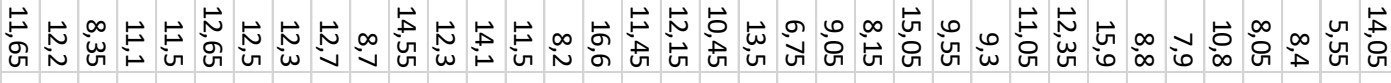

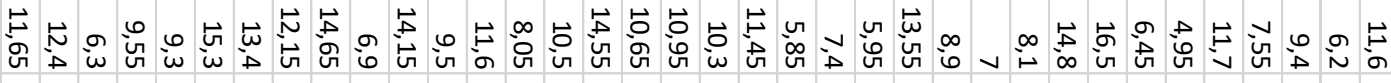

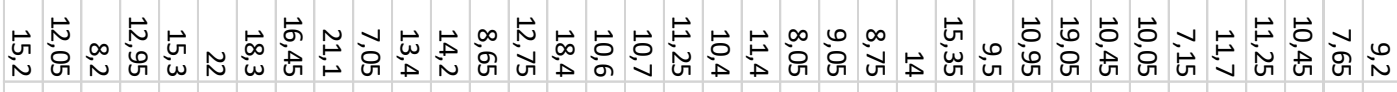

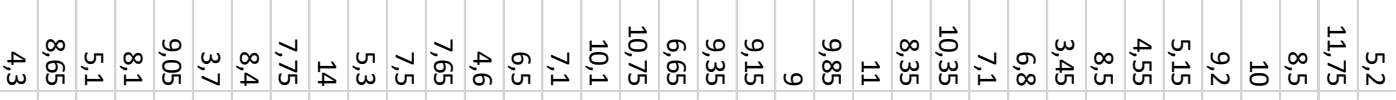

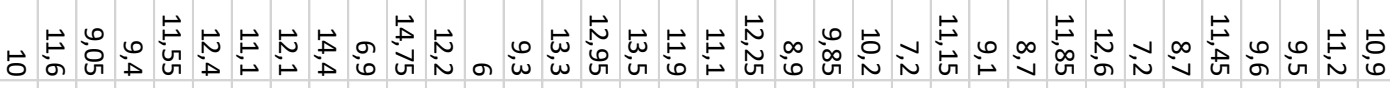

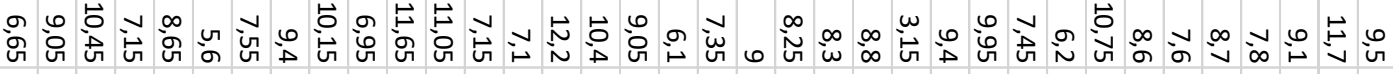

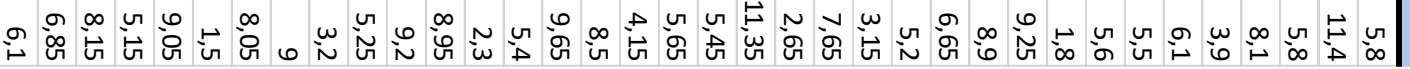
Tabelle A.4 Tabellarische Darstellung der in DVT-Aufnahmen gemessenen Werte 


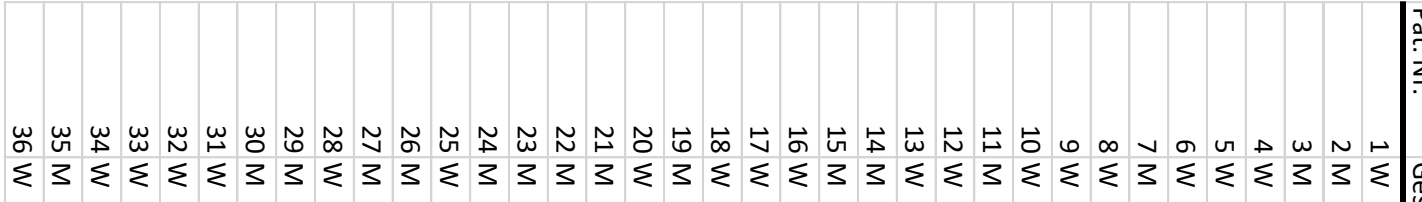

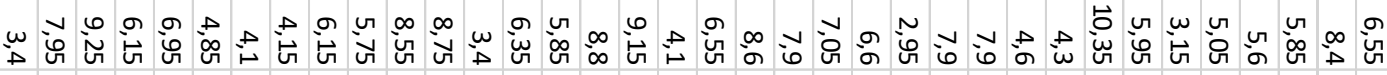

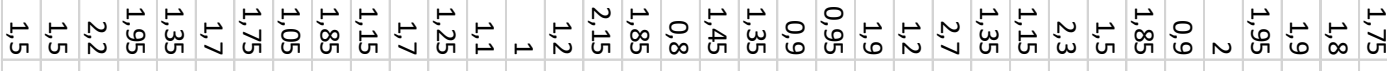

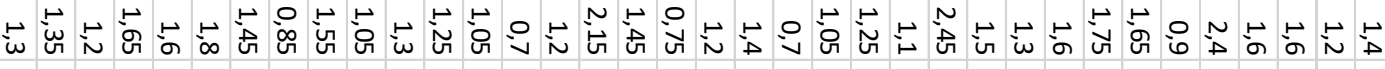

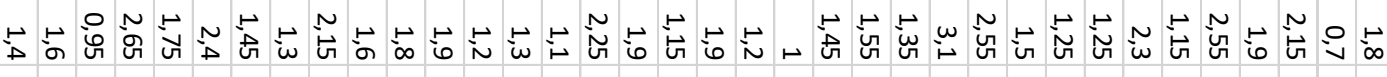

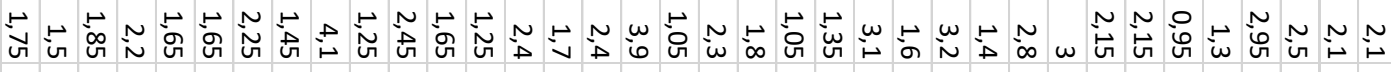

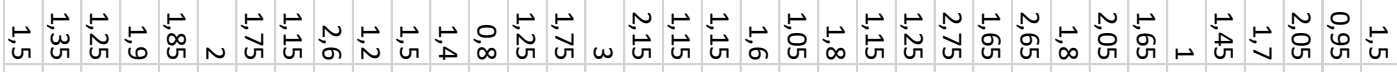

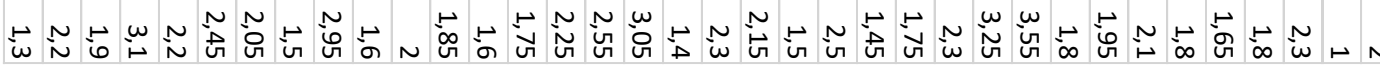
Tabelle A.5 Tabellarische Darstellung der in DVT-Aufnahmen gemessenen Werte 


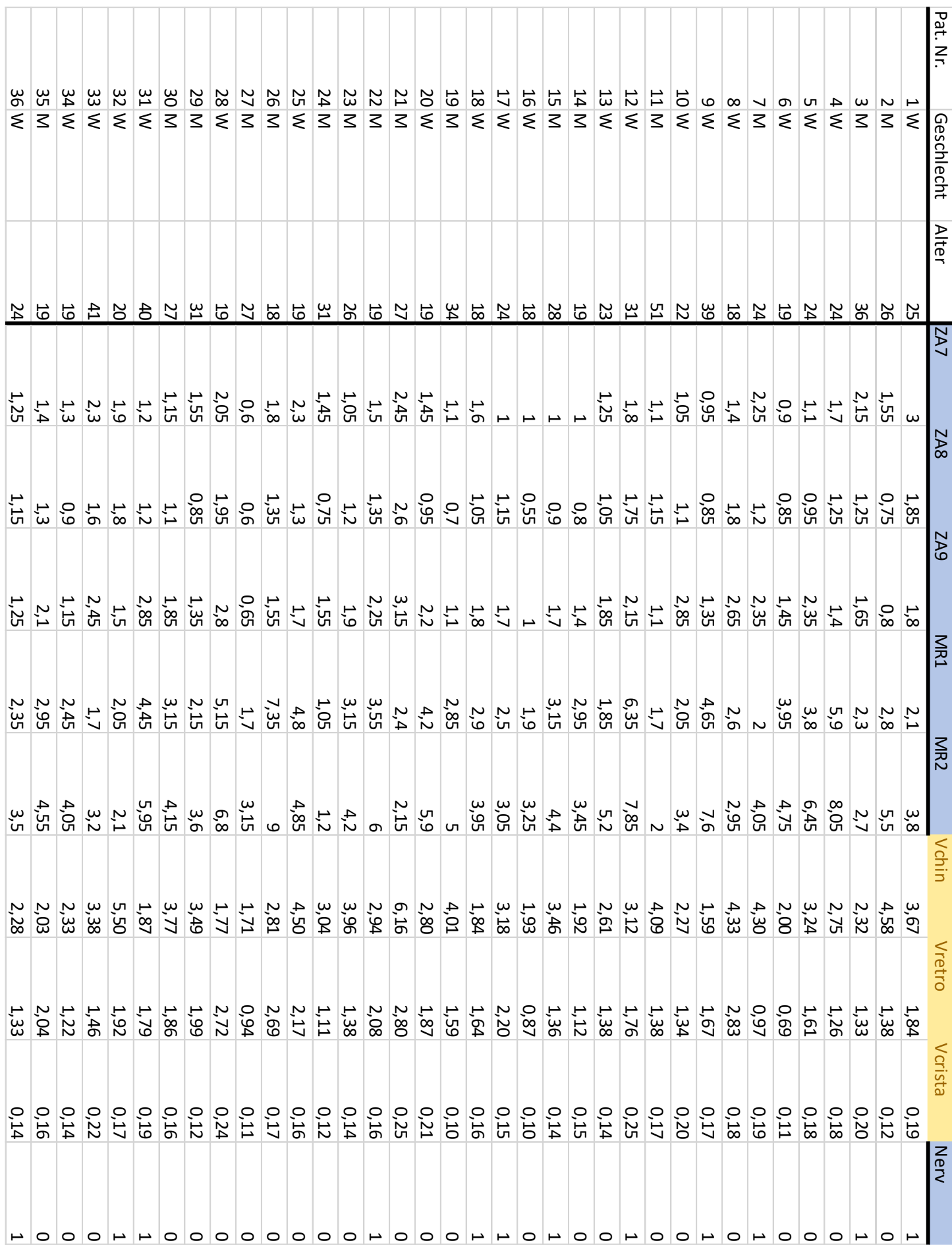




\section{Literaturverzeichnis}

Acocella A, Bertolai R, Colafranceschi M, Sacco R (2010): Clinical, histological and histomorphometric evaluation of the healing of mandibular ramus bone block grafts for alveolar ridge augmentation before implant placement. J Craniomaxillofac Surg 38, 222-230

Aichelmann-Reidy ME, Yukna RA (1998): Bone replacement grafts. The bone substitutes. Dent Clin North Am 42, 491-503

Akiba T (2013): Utility of three-dimensional computed tomography in general thoracic surgery. Gen Thorac Cardiovasc Surg 61, 676-684

Albrektsson T, Johansson C (2001): Osteoinduction, osteoconduction and osseointegration. Eur Spine J 10, 96-101

Altiparmak N, Akdeniz BS, Akdeniz SS, Uçkan S (2017): Changes in the lower lip soft tissue after bone graft harvesting from the mandibular symphysis. Int J Oral Maxillofac Surg 46, 129133

Amarnath GS, Kumar U, Hilal M, Muddugangadhar BC, Anshuraj K, Shruthi CS (2015): Comparison of cone beam computed tomography, orthopantomography with direct ridge mapping for pre-surgical planning to place implants in cadaveric mandibles: an ex-vivo study. J Int Oral Health $\underline{7}, 38-42$

Andersen K, Nørholt SE, Knudsen J, Küseler A, Jensen J (2014): Donor site morbidity after reconstruction of alveolar bone defects with mandibular symphyseal bone grafts in cleft patients--111 consecutive patients. Int J Oral Maxillofac Surg $\underline{43}, 428-432$

Anderson LC, Kosinski TF, Mentag PJ (1991): A review of the intraosseous course of the nerves of the mandible. J Oral Implantol 17, 394-403

Arai Y, Tammisalo E, Iwai K, Hashimoto K, Shinoda K (1999): Development of a compact computed tomographic apparatus for dental use. Dentomaxillofac Radiol 28, 245-248

Araújo MG, Lindhe J (2009): Ridge alterations following tooth extraction with and without flap elevation: an experimental study in the dog. Clin Oral Implants Res 20, 545-549

Araújo MG, Silva CO, Misawa M, Sukekava F (2015): Alveolar socket healing: what can we learn? Periodontol $2000 \underline{68}, 122-134$

Beckmann G, Wingberg J, Hasund A (1983): Computerunterstützte Kephalometrie in der BergenTechnik. Fortschritte der Kieferorthopädie 44, 359-369

Beckmann SH, Kuitert RB, Prahl-Andersen B, Segner D, The RP, Tuinzing DB (1998a): Alveolar and skeletal dimensions associated with lower face height. Am J Orthod Dentofacial Orthop $\underline{113}, 498-506$

Beckmann SH, Kuitert RB, Prahl-Andersen B, Segner D, The RP, Tuinzing DB (1998b): Alveolar and skeletal dimensions associated with overbite. Am J Orthod Dentofacial Orthop $\underline{113}$, 443452 
Benavides E, Rios HF, Ganz SD, An CH, Resnik R, Reardon GT, Feldman SJ, Mah JK, Hatcher D, Kim MJ et al. (2012): Use of cone beam computed tomography in implant dentistry: the International Congress of Oral Implantologists consensus report. Implant Dent 21, 78-86

Berco M, Rigali PH, Miner RM, DeLuca S, Anderson NK, Will LA (2009): Accuracy and reliability of linear cephalometric measurements from cone-beam computed tomography scans of a dry human skull. Am J Orthod Dentofacial Orthop $\underline{136}$, 17-18

Bjork A (1963): Variations in the growth pattern of the human mandible: Longitudinal radiographic study by the implant method. J Dent Res $\underline{42}, 400-411$

Björk A (1969): Prediction of mandibular growth rotation. Am J Orthod $\underline{55}, 585-599$

Bland JM, Altman DG (1986): Statistical methods for assessing agreement between two methods of clinical measurement. Lancet $\underline{1}, 307-310$

Bogaerts E, van der Vekens E, Verhoeven G, de Rooster H, van Ryssen B, Samoy Y, Putcuyps I, van Tilburg J, Devriendt N, Weekers F et al. (2018): Intraobserver and interobserver agreement on the radiographical diagnosis of canine cranial cruciate ligament rupture. Vet Rec 182,484

Brånemark PI, Hansson BO, Adell R, Breine U, Lindström J, Hallén O, Ohman A (1977): Osseointegrated implants in the treatment of the edentulous jaw. Experience from a 10-year period. Scand J Plast Reconstr Surg Suppl 16, 1-132

Braut V, Bornstein MM, Belser U, Buser D (2011): Thickness of the anterior maxillary facial bone wall-a retrospective radiographic study using cone beam computed tomography. Int J Periodontics Restorative Dent 31, 125-131

Broadbent BH (1931): A new x-ray technique and its application to orthodontia. The Angle Orthodontist $\underline{1}, 45-66$

Bruks A, Enberg K, Nordqvist I, Hansson AS, Jansson L, Svenson B (1999): Radiographic examinations as an aid to orthodontic diagnosis and treatment planning. Swed Dent J $\underline{23}, 77-85$

Buck BE, Malinin TI, Brown MD (1989): Bone transplantation and human immunodeficiency virus. An estimate of risk of acquired immunodeficiency syndrome (AIDS). Clin Orthop Relat Res $\underline{240}, 129-136$

Bundesamt für Strahlenschutz - Röntgen - Röntgendiagnostik: Häufigkeit und Strahlenexposition. https://www.bfs.de/DE/themen/ion/anwendung-medizin/diagnostik/roentgen/haeufigkeit-exposition.html; Zugriff am 21.02.2018

Capelli M (2003): Autogenous bone graft from the mandibular ramus: A technique for bone augmentation. Int J Periodontics Restorative Dent 23, 277-285

Cavalcanti MG, Vannier MW (1998): Quantitative analysis of spiral computed tomography for craniofacial clinical applications. Dentomaxillofac Radiol 27, 344-350

Cavalcanti MGP, Rocha SS, Vannier MW (2004): Craniofacial measurements based on 3D-CT volume rendering: implications for clinical applications. Dentomaxillofac Radiol 33, 170-176

Celik E, Polat-Ozsoy O, Toygar Memikoglu TU (2009): Comparison of cephalometric measurements with digital versus conventional cephalometric analysis. Eur J Orthod $\underline{31}, 241-246$ 
Clavero J, Lundgren S (2003): Ramus or chin grafts for maxillary sinus inlay and local onlay augmentation: comparison of donor site morbidity and complications. Clin Implant Dent Relat Res $\underline{5}, 154-160$

Cunningham SJ, Johal A (2015): Orthognathic correction of dento-facial discrepancies. Br Dent J 218, 167-175

Dahlström L, Lindvall AM (1996): Assessment of temporomandibular joint disease by panoramic radiography: reliability and validity in relation to tomography. Dentomaxillofac Radiol $\underline{25}$, 197-201

Deguchi T, Katashiba S, Inami T, Foong KWC, Huak CY (2010): Morphologic quantification of the maxilla and the mandible with cone-beam computed tomography. Am J Orthod Dentofacial Orthop 137, 218-222

Delloye C, Cnockaert N, Cornu O (2003): Bone substitutes in 2003: an overview. Acta Orthop Belg $\underline{69}, 1-8$

Devereux L, Moles D, Cunningham SJ, McKnight M (2011): How important are lateral cephalometric radiographs in orthodontic treatment planning? Am J Orthod Dentofacial Orthop 139 , $175-181$

Douglass CW, Valachovic RW, Wijesinha A, Chauncey HH, Kapur KK, McNeil BJ (1986): Clinical efficacy of dental radiography in the detection of dental caries and periodontal diseases. Oral Surg Oral Med Oral Pathol 62, 330-339

Fallon SD, Fritz GW, Laskin DM (2006): Panoramic imaging of the temporomandibular joint: An experimental study using cadaveric skulls. J Oral Maxillofac Surg 64, 223-229

Fuentes R, Flores T, Navarro P, Salamanca C, Beltrán V, Borie E (2015): Assessment of buccal bone thickness of aesthetic maxillary region: a cone-beam computed tomography study. J Periodontal Implant Sci $\underline{45}$, 162-168

Garg AK, Terheyden H: Knochen: Biologie, Gewinnung, Transplantation in der zahnärztlichen Implantologie (Quintessenz-Bibliothek). Quintessenz-Verlag, Berlin 2006

Gellrich NC, Held U, Schoen R, Pailing T, Schramm A, Bormann KH (2007): Alveolar zygomatic buttress: A new donor site for limited preimplant augmentation procedures. J Oral Maxillofac Surg $\underline{65}, 275-280$

Gellrich NC, Bormann KH, Tehranchian S, Kokemüller H, Suarez-Cunqueiro MM (2013): Containment and contouring (CoCoon) technique: A biologically adequate approach to less invasive autogenous preimplant augmentation of bone. Br J Oral Maxillofac Surg 51, 880-886

Gong X, Zhang X, Song Y, Li Y, Pang X, Li S (2007): [Effects of immunosuppressive treatment in prevention of calcification in aortic valved homograft: experiment with rats]. Zhonghua Yi Xue Za Zhi 87, 2132-2135

Grobben AH, Steele PJ, Somerville RA, Taylor DM (2004): Inactivation of the bovine-spongiformencephalopathy (BSE) agent by the acid and alkaline processes used in the manufacture of bone gelatine. Biotechnol Appl Biochem 39, 329-338 
Grünheid T, Kolbeck Schieck JR, Pliska BT, Ahmad M, Larson BE (2012): Dosimetry of a cone-beam computed tomography machine compared with a digital $\mathrm{x}$-ray machine in orthodontic imaging. Am J Orthod Dentofacial Orthop 141, 436-443

Hahn W, Klotz S, Gruber RM (Hrsg.): Knochenaufbau in der zahnärztlichen Implantologie: Allgemeine und operative Grundlagen. Spitta Verlag, Balingen 2008

Hahn W, Klotz S, Gruber RM (Hrsg.): Knochenaufbau in der zahnärztlichen Implantologie: Weiterführende operative Techniken. Spitta Verlag, Balingen 2008

Handelman CS (1996): The anterior alveolus: its importance in limiting orthodontic treatment and its influence on the occurrence of iatrogenic sequelae. Angle Orthod 66, 95-109

Hofrath H (1931): Die Bedeutung der Röntgenfern- und Abstandsaufnahme für die Diagnostik der Kieferanomalien. Fortschritte der Orthodontik 1, 232-258

Hoppenreijs TJ, Nijdam ES, Freihofer HP (1992): The chin as a donor site in early secondary osteoplasty: a retrospective clinical and radiological evaluation. J Craniomaxillofac Surg $\underline{20}$, 119-124

Hurlburt CE, Wuehrmann AH (1976): Comparison of interproximal carious lesion detection in panoramic and standard intraoral radiography. J Am Dent Assoc 93, 1154-1158

Kabak SL, Zhuravleva NV, Melnichenko YM, Savrasova NA (2017): Study of the mandibular incisive canal anatomy using cone beam computed tomography. Surg Radiol Anat $\underline{39}$, 647-655

Kaeppler G, Mast M (2012): Indications for cone-beam computed tomography in the area of oral and maxillofacial surgery. Int J Comput Dent $\underline{15}, 271-286$

Kainulainen VT, Sàndor GKB, Oikarinen KS, Clokie CML (2002): Zygomatic bone: an additional donor site for alveolar bone reconstruction. Technical note. Int J Oral Maxillofac Implants $\underline{17}$ $723-728$

Kalabalik F, Aytuğar E (2019): Localization of the Mandibular Canal in a Turkish Population: a Retrospective Cone-Beam Computed Tomography Study. J Oral Maxillofac Res $\underline{10}$, e2

Kamburoglu K, Kolsuz E, Murat S, Yüksel S, Ozen T (2012): Proximal caries detection accuracy using intraoral bitewing radiography, extraoral bitewing radiography and panoramic radiography. Dentomaxillofac Radiol 41, 450-459

Kapila S, Conley RS, Harrell WE (2011): The current status of cone beam computed tomography imaging in orthodontics. Dentomaxillofac Radiol 40, 24-34

Katayama K, Yamaguchi T, Sugiura M, Haga S, Maki K (2014): Evaluation of mandibular volume using cone-beam computed tomography and correlation with cephalometric values. Angle Orthod $\underline{84}, 337-342$

Katheria BC, Kau CH, Tate R, Chen JW, English J, Bouquot J (2010): Effectiveness of impacted and supernumerary tooth diagnosis from traditional radiography versus cone beam computed tomography. Pediatr Dent 32, 304-309

Khorshidi H, Raoofi S, Ghapanchi J, Shahidi S, Paknahad M (2017): Cone Beam Computed Tomographic Analysis of the Course and Position of Mandibular Canal. J Maxillofac Oral Surg $\underline{16}$, 306-311 
Krarup S, Darvann TA, Larsen P, Marsh JL, Kreiborg S (2005): Three-dimensional analysis of mandibular growth and tooth eruption. J Anat 207, 669-682

Kübler NR, Will C, Depprich R, Betz T, Reinhart E, Bill JS, Reuther JF (1999): Vergleichende Untersuchungen zur Sinusbodenelevation mit autogenem oder allogenem Knochengewebe. Mund Kiefer Gesichtschir 3, 53-60

Kuitert R, Beckmann S, van Loenen M, Tuinzing B, Zentner A (2006): Dentoalveolar compensation in subjects with vertical skeletal dysplasia. Am J Orthod Dentofacial Orthop $\underline{129}, 649-657$

Lenza MA, Carvalho AA de, Lenza EB, Lenza MG, Torres HM de, Souza JB de (2015): Radiographic evaluation of orthodontic treatment by means of four different cephalometric superimposition methods. Dental Press J Orthod 20, 29-36

Levine MH, Goddard AL, Dodson TB (2007): Inferior alveolar nerve canal position: a clinical and radiographic study. J Oral Maxillofac Surg $\underline{65}, 470-474$

Liu DX, Wang CL, Liu L, Dong ZY, Ke HF, Yu ZY (2006): [The accuracy of 3D-CT volume rendering for craniofacial linear measurements]. Shanghai Kou Qiang Yi Xue 15, 517-520

Lopez-Capp TT, Rynn C, Wilkinson C, de Paiva LAS, Michel-Crosato E, Biazevic MGH (2017): Discriminant analysis of mandibular measurements for the estimation of sex in a modern Brazilian sample. Int J Legal Med 132, 843-851

Loubele M, Bogaerts R, van Dijck E, Pauwels R, Vanheusden S, Suetens P, Marchal G, Sanderink G, Jacobs $R$ (2009): Comparison between effective radiation dose of CBCT and MSCT scanners for dentomaxillofacial applications. Eur J Radiol $\underline{71}, 461-468$

Ludlow JB, Davies-Ludlow LE, Brooks SL, Howerton WB (2006): Dosimetry of 3 CBCT devices for oral and maxillofacial radiology: CB Mercuray, NewTom 3G and i-CAT. Dentomaxillofac Radiol $\underline{35}$, 219-226

Ludlow JB, Davies-Ludlow LE, White SC (2008): Patient risk related to common dental radiographic examinations: the impact of 2007 International Commission on Radiological Protection recommendations regarding dose calculation. J Am Dent Assoc 139, 1237-1243

Misch CM (2000): Use of the mandibular ramus as a donor site for onlay bone grafting. J Oral Implantol 26, 42-49

Misch KA, Yi ES, Sarment DP (2006): Accuracy of cone beam computed tomography for periodontal defect measurements. J Periodontol $\underline{77}, 1261-1266$

Mozzo P, Procacci C, Tacconi A, Martini PT, Andreis IA (1998): A new volumetric CT machine for dental imaging based on the cone-beam technique: preliminary results. Eur Radiol $\underline{8}$, 1558-1564

Nasr HF, Aichelmann-Reidy ME, Yukna RA (1999): Bone and bone substitutes. Periodontol 2000 $\underline{19}, 74-86$

Nielsen IL (2011): Analysis of general facial growth, maxillary and mandibular growth and treatment changes („Structural analysis“). Int Orthod 9 , 388-409

Nkenke E, Neukam FW (2014): Autogenous bone harvesting and grafting in advanced jaw resorption: morbidity, resorption and implant survival. Eur J Oral Implantol Z, 203-217 
Ohba T, Takata Y, Ansai T, Morimoto Y, Tanaka T, Kito S, Awano S, Akifusa S, Takehara T (2003): Evaluation of calcified carotid artery atheromas detected by panoramic radiograph among 80-year-olds. Oral Surg Oral Med Oral Pathol Oral Radiol Endod 96, 647-650

Okşayan R, Aktan AM, Sökücü O, Haştar E, Ciftci ME (2012): Does the panoramic radiography have the power to identify the gonial angle in orthodontics? ScientificWorldJournal 2012, 219708

Omoumi P, Michoux N, Larbi A, Lacoste L, Lecouvet FE, Perlepe V, Vande Berg BC (2017): Multirater agreement for grading the femoral and tibial cartilage surface lesions at $\mathrm{CT}$ arthrography and analysis of causes of disagreement. Eur J Radiol 88, 95-101

Panagiotidis DG, Witt PDE (2005): Der individualisierte ANB-Winkel. Fortschritte der Kieferorthopädie $\underline{38}, 408-416$

Pasler FA: Zahnärztliche Radiologie. 6. Auflage; Georg Thieme Verlag, Stuttgart New York 2017

Pauwels R (2015): Cone beam CT for dental and maxillofacial imaging: dose matters. Radiat Prot Dosimetry $\underline{165}, 156-161$

Pereira-Maciel P, Tavares-de-Sousa E, Oliveira-Sales MA (2015): The mandibular incisive canal and its anatomical relationships: A cone beam computed tomography study. Med Oral Patol Oral Cir Bucal 20, e723-e728

Pikos MA (1995): Facilitating implant placement with chin grafts as donor sites for maxillary bone augmentation--Part I. Dent Implantol Update $\underline{6}$, 89-92

Pires CA, Bissada NF, Becker JJ, Kanawati A, Landers MA (2012): Mandibular incisive canal: cone beam computed tomography. Clin Implant Dent Relat Res $\underline{14}$, 67-73

Provedel LF, Pimentel BNA da S, Jesus TM de, Lacerda APS, Rubira-Bullen IRF, Sarmento VA (2016): Effects of image compression on linear measurements of digital panoramic radiographs. Braz Dent J 27, 757-760

Quiudini PR, Pozza DH, Pinto ADS, de Arruda MF, Guimarães AS (2017): Differences in bite force between dolichofacial and brachyfacial individuals: Side of mastication, gender, weight and height. J Prosthodont Res $\underline{61}$, 283-289

Radhakrishnan PD, Sapna Varma NK, Vallikat Velath Ajith (2017): Dilemma of gonial angle measurement: Panoramic radiograph or lateral cephalogram. Imaging Sci Dent 47, 93-97

Reininger D, Cobo-Vázquez C, Monteserín-Matesanz M, López-Quiles J (2016): Complications in the use of the mandibular body, ramus and symphysis as donor sites in bone graft surgery. A systematic review. Med Oral Patol Oral Cir Bucal 21, e241-e249

Rich J, Golden BA, Phillips C (2014): Systematic review of preoperative mandibular canal position as it relates to postoperative neurosensory disturbance following the sagittal split ramus osteotomy. Int J Oral Maxillofac Surg 43, 1076-1081

Ricketts RM (1972): The value of cephalometrics and computerized technology. Angle Orthod $\underline{42}$, 179-199

Rogers GF, Greene AK (2012): Autogenous bone graft: basic science and clinical implications. J Craniofac Surg 23, 323-327 
Rubens BC, West RA (1989): Ptosis of the chin and lip incompetence: consequences of lost mentalis muscle support. J Oral Maxillofac Surg 느, 359-366

Sadek MM, Sabet NE, Hassan IT (2015): Alveolar bone mapping in subjects with different vertical facial dimensions. Eur J Orthod 37 , 194-201

Samartzis D, Nishi N, Hayashi M, Cologne J, Cullings HM, Kodama K, Miles EF, Funamoto S, Suyama A, Soda M, Kasagi F (2011): Exposure to ionizing radiation and development of bone sarcoma: new insights based on atomic-bomb survivors of Hiroshima and Nagasaki. J Bone Joint Surg Am 93, 1008-1015

Samartzis D, Nishi N, Cologne J, Funamoto S, Hayashi M, Kodama K, Miles EF, Suyama A, Soda M, Kasagi $F(2013)$ : lonizing radiation exposure and the development of soft-tissue sarcomas in atomic-bomb survivors. J Bone Joint Surg Am 95, 222-229

Santoro M, Jarjoura K, Cangialosi TJ (2006): Accuracy of digital and analogue cephalometric measurements assessed with the sandwich technique. Am J Orthod Dentofacial Orthop $\underline{129}$, 345-351

Sayinsu K, Isik F, Trakyali G, Arun T (2007): An evaluation of the errors in cephalometric measurements on scanned cephalometric images and conventional tracings. Eur J Orthod 29, 105108

Schropp L, Wenzel A, Kostopoulos L, Karring T (2003): Bone healing and soft tissue contour changes following single-tooth extraction: a clinical and radiographic 12-month prospective study. Int J Periodontics Restorative Dent 23, 313-323

Schulze R, Heil U, Gross D, Bruellmann DD, Dranischnikow E, Schwanecke U, Schoemer E (2011): Artefacts in CBCT: a review. Dentomaxillofac Radiol 40, 265-273

Schünke M, Schulte E, Schumacher U (Hrsg.): Prometheus - Lernatlas der Anatomie: Kopf, Hals und Neuroanatomie. 2. Auflage; Georg Thieme Verlag, Stuttgart, New York 2009

Schwartz Z, Weesner T, van Dijk S, Cochran DL, Mellonig JT, Lohmann CH, Carnes DL, Goldstein M, Dean DD, Boyan BD (2000): Ability of deproteinized cancellous bovine bone to induce new bone formation. J Periodontol $\underline{71}, 1258-1269$

Segner D, Hasund A: Individualisierte Kephalometrie. 3. Auflage; Segner, Hamburg 1998

Signorelli L, Patcas R, Peltomäki T, Schätzle M (2016): Radiation dose of cone-beam computed tomography compared to conventional radiographs in orthodontics. J Orofac Orthop $\underline{77}, 9$ 15

Silva FMS, Cortez ALV, Moreira RWF, Mazzonetto R (2006): Complications of intraoral donor site for bone grafting prior to implant placement. Implant Dent $\underline{15}$, 420-426

Sogal A, Tofe AJ (1999): Risk assessment of bovine spongiform encephalopathy transmission through bone graft material derived from bovine bone used for dental applications. J Periodontol $\underline{70}, 1053-1063$

Strietzel FP, Reichart PA, Graf HL (2007): Lateral alveolar ridge augmentation using a synthetic nano-crystalline hydroxyapatite bone substitution material (Ostim): preliminary clinical and histological results. Clin Oral Implants Res $\underline{18}, 743-751$ 
Stübinger S, Robertson A, Zimmerer KS, Leiggener C, Sader R, Kunz C (2006): Piezoelectric harvesting of an autogenous bone graft from the zygomaticomaxillary region: case report. Int J Periodontics Restorative Dent 26, 453-457

Stübinger S, Stricker A, Berg BI (2015): Piezosurgery in implant dentistry. Clin Cosmet Investig Dent $\underline{7}, 115-124$

Taylor DM, McConnell I, Fernie K (1996): The effect of dry heat on the ME7 strain of mouse-passaged scrapie agent. J Gen Virol $\underline{77}$, 3161-3164

Terry GL, Noujeim M, Langlais RP, Moore WS, Prihoda TJ (2016): A clinical comparison of extraoral panoramic and intraoral radiographic modalities for detecting proximal caries and visualizing open posterior interproximal contacts. Dentomaxillofac Radiol 45, 20150159

Valachovic RW, Douglass CW, Reiskin AB, Chauncey HH, McNeil BJ (1986): The use of panoramic radiography in the evaluation of asymptomatic adult dental patients. Oral Surg Oral Med Oral Pathol $\underline{61}, 289-296$

van der Stelt PF (2005): Filmless imaging: the uses of digital radiography in dental practice. J Am Dent Assoc 136, 1379-1387

van der Stelt PF (2016): [Panoramic radiographs in dental diagnostics]. Ned Tijdschr Tandheelkd $\underline{123}, 181-187$

Varol A, Atali O, Sipahi A, Basa S (2016): Implant rehabilitation for extremely atrophic maxillae (Cawood Type VI) with Le Fort I downgrafting and autogenous iliac block grafts: A 4-year follow-up study. Int J Oral Maxillofac Implants 31, 1415-1422

Vera C, De Kok IJ, Reinhold D, Limpiphipatanakorn P, Yap AKW, Tyndall D, Cooper LF (2012): Evaluation of buccal alveolar bone dimension of maxillary anterior and premolar teeth: a cone beam computed tomography investigation. Int J Oral Maxillofac Implants 27, 1514-1519

Vercellotti T, De Paoli S, Nevins M (2001): The piezoelectric bony window osteotomy and sinus membrane elevation: introduction of a new technique for simplification of the sinus augmentation procedure. Int J Periodontics Restorative Dent 21, 561-567

Visser H, Rödig T, Hermann KP (2001): Dose reduction by direct-digital cephalometric radiography. Angle Orthod $\underline{71}, 159-163$

von Garrel T, Gotzen L (1998): Allogene Knochentransplantation und Knochenbanking. Unfallchirurg $\underline{101}, 713-727$

Wang H, Shen J, Yu M, Chen X, Jiang Q, He F (2014): Analysis of facial bone wall dimensions and sagittal root position in the maxillary esthetic zone: a retrospective study using cone beam computed tomography. Int J Oral Maxillofac Implants 29, 1123-1129

Wedler S, Tränkmann J, Lisson JA (2006): Treatment outcome in angle class II, division 1 patients in pre-puberty and puberty after jumping-the-bite appliance. J Orofac Orthop 67, 105-115

Weibull L, Widmark G, Ivanoff CJ, Borg E, Rasmusson L (2009): Morbidity after chin bone harvesting: a retrospective long-term follow-up study. Clin Implant Dent Relat Res $\underline{11}$, 149-157

Wenz B, Oesch B, Horst M (2001): Analysis of the risk of transmitting bovine spongiform encephalopathy through bone grafts derived from bovine bone. Biomaterials $\underline{22}, 1599-1606$ 
Woelber JP, Fleiner J, Rau J, Ratka-Krüger P, Hannig C (2018): Accuracy and usefulness of CBCT in periodontology: a systematic review of the literature. Int J Periodontics Restorative Dent 38, 289-297

Yates DM, Brockhoff HC, Finn R, Phillips C (2013): Comparison of intraoral harvest sites for corticocancellous bone grafts. J Oral Maxillofac Surg $\underline{71}, 497-504$

Yavuz MS, Buyukkurt MC, Tozoglu S, Dagsuyu IM, Kantarci M (2009): Evaluation of volumetry and density of mandibular symphysis bone grafts by three-dimensional computed tomography. Dent Traumatol $\underline{25}, 475-479$

Zangouei-Booshehri M, Aghili HA, Abasi M, Ezoddini-Ardakani F (2012): Agreement between panoramic and lateral cephalometric radiographs for measuring the gonial angle. Iran J Radiol $\underline{9}, 178-182$

Zeltner M, Flückiger LB, Hämmerle CHF, Hüsler J, Benic GI (2016): Volumetric analysis of chin and mandibular retromolar region as donor sites for cortico-cancellous bone blocks. Clin Oral Implants Res 27, 999-1004

Zerbo IR, de Lange GL, Joldersma M, Bronckers ALJ, Burger EH (2003): Fate of monocortical bone blocks grafted in the human maxilla: a histological and histomorphometric study. Clin Oral Implants Res $\underline{14}$, 759-766 


\section{Danksagung}

Mein größter Dank gilt meinem Doktorvater PD Dr. W. Hahn, der mir diese Arbeit nicht nur freundlich überließ, sondern durch seine gute Betreuung überhaupt erst ermöglichte. Die geforderte und geförderte Präzision empfinde ich als persönliche Bereicherung.

Ich möchte auch PD Dr. T. Rödig für Ihre wertvolle Zeit bei den produktiven Zusammentreffen im Rahmen der Ko-Betreuung danken.

Zudem danke ich der Abteilung für Mund-, Kiefer- und Gesichtschirurgie der Universitätsmedizin Göttingen für die Nutzung der Räumlichkeiten und Röntgenaufnahmen.

Abschließend bedanke ich mich bei Frau Maria Stark für Ihre Unterstützung im Rahmen des betreuten Arbeitens im Institut für medizinische Statistik der Universitätsmedizin Göttingen. 